\title{
Time-Symmetry Breaking in Turbulent Multi-Particle Dispersion
}

\author{
Dissertation \\ for the award of the degree "Doctor rerum naturalium" \\ of the Georg-August-Universität Göttingen \\ within the doctoral program \\ Physics of Biological and Complex Systems \\ of the Georg-August University School of Science (GAUSS)
}

submitted by

Jennifer Jucha née Mutschall

from Koblenz

Göttingen, 2014 


\section{Thesis Committee:}

Prof. Dr. Eberhard Bodenschatz

Laboratory for Fluid Dynamics, Pattern Formation, and Biocomplexity,

Max Planck Institute for Dynamics and Self-Organization

Prof. Dr. Annette Zippelius

Institut für Theoretische Physik, Georg-August-Universität Göttingen

Prof. Dr. Jens Niemeyer

Institut für Astrophysik, Georg-August-Universität Göttingen

\section{Members of the Examination Board:}

Referee: Prof. Dr. Eberhard Bodenschatz

Laboratory for Fluid Dynamics, Pattern Formation, and Biocomplexity,

Max Planck Institute for Dynamics and Self-Organization

2nd Referee: Prof. Dr. Annette Zippelius

Institut für Theoretische Physik, Georg-August-Universität Göttingen

\section{Further Members of the Examination Board:}

Prof. Dr. Jens Niemeyer

Institut für Astrophysik, Georg-August-Universität Göttingen

\section{Dr. Haitao Xu}

Laboratory for Fluid Dynamics, Pattern Formation, and Biocomplexity,

Max Planck Institute for Dynamics and Self-Organization

\section{Prof. Dr. Gert Lube}

Institut für Numerische und Angwandte Mathematik, Georg-August-Universität Göttingen

\section{Dr. Eleni Katifori}

Physics of Biological Organization, Max Planck Institute for Dynamics and Self-Organization 
"Turbulence is the most important unsolved problem of classical physics."

Richard P. Feynman, The Feynman Lectures on Physics, 1964 

1. Introduction and Theory 9

1.1. The Governing Equations . . . . . . . . . . . . . . . . . . . . 9

1.2. Kolmogorov's Theory (1941) . . . . . . . . . . . . . . . . . . . 12

1.3. Turbulent Dispersion . . . . . . . . . . . . . . . . . . . 14

2. Experimental Methods 23

2.1. Lagrangian Particle Tracking . . . . . . . . . . . . . . . . . . . . 23

2.2. Velocity and Acceleration Computation . . . . . . . . . . . . 32

2.3. Experimental Setup . . . . . . . . . . . . . . . . . . . . . . . . . . . . . . . . . .

2.4. Datasets . . . . . . . . . . . . . . . . . . 39

2.5. Measurement Uncertainties . . . . . . . . . . . . . . . . . . . 43

3. Two-Particle Dispersion $\quad 47$

3.1. Short-Time Expansion . . . . . . . . . . . . . . . . . . . 47

3.2. Data Analysis . . . . . . . . . . . . . . . . . . . . . 49

3.3. Experimental Results . . . . . . . . . . . . . . . . . . . . 50

4. Four-Particle Dispersion $\quad \mathbf{5 7}$

4.1. Short-Time Expansion . . . . . . . . . . . . . . . . . . . 57

4.2. Data Analysis . . . . . . . . . . . . . . . . . . . 61

4.3. Experimental Results . . . . . . . . . . . . . . . . . 63 
5. The Effect of Polymers $\quad \mathbf{7 1}$

5.1. Preparation of the Experiment . . . . . . . . . . . . 71

5.2. Effect of Polymers on the Energy Cascade . . . . . . . . . . . . . . 74

5.3. Two-Particle Dispersion . . . . . . . . . . . . . . . . . . 78

5.4. Four-Particle Dispersion _. . . . . . . . . . . . . . . 80

$\begin{array}{lr}\text { 6. Discussion and Perspectives } & \mathbf{8 5}\end{array}$

$\begin{array}{ll}\text { Appendix } & \mathbf{8 9}\end{array}$

A. Supplementary Figures and Derivations _... . . . . . . . . . . . . 89

B. Flow Properties . . . . . . . . . . . . . . . . . . . . . . 97

C. Data Formats . . . . . . . . . . . . . . . . 102

$\begin{array}{lr}\text { Bibliography } & 105\end{array}$

$\begin{array}{lr}\text { Curriculum Vitae } & 113\end{array}$

$\begin{array}{lr}\text { Acknowledgments } & 117\end{array}$ 


\section{Motivation}

Throughout the centuries, many prestigious physicists and mathematicians have tried to describe the dynamics of turbulent flows and predict their evolution (for a historical overview, see e.g Lumley \& Yaglom 2001 and Davidson et al. 2011). In fluid dynamics, the term "turbulence" usually describes a special state of a continuous medium in which many interacting degrees of freedom are excited, leading to strong and chaotic temporal and spatial fluctuations in velocity, pressure and other flow properties. This behavior can be found in the atmospheric flows determining our weather and climate, water flows in rivers and oceans, right down to the coffee we drink and the air we breathe (Shaw 2003, Bodenschatz et al. 2010, Naveira Garabato et al. 2004, Shraiman \& Siggia 2000). Accordingly, turbulent flows are of large interest from a practical viewpoint and many studies are conducted on the effect of turbulence on drag, combustion, and sedimentation, to name only a few. From a more fundamental perspective, the high number of excited degrees of freedom in a turbulent flow lead to a complex physical state far from equilibrium, not characterizable with the tools of equilibrium statistical mechanics. New theoretical approaches are needed to describe the multitude of fascinating phenomena observed in experimental and natural flows.

One of those phenomena is the time irreversibility of turbulent flows. This can be easily seen when comparing the mixing of substances in turbulent and Stokes flow. If a blotch of colored corn syrup is injected into a Couette cell filled with corn syrup and the inner cylinder is rotated, the two components are spread into thin layers. The formation of the layers can be easily reversed, however, by turning the inner cylinder the other way around until the blotch has recollected at its original position, with just a slight blurring due to diffusion (Heller 1960). If milk is stirred into coffee, on the other hand, turbulent flow generates much more complicated, interwoven layer structures of the two substances (Ottino 1989b, Dimotakis 2005). Reversing the stirring direction will not untangle the produced layers but rather enhance the complexity of their structure, and the initial condition of two unmixed fluids can never be reproduced. The irreversibility of turbulent flows arises from instabilities that lead to a flux of energy through scales. For a three-dimensional flow, the spatial scale at which energy is injected, $L$, is much larger than the scale at which it is dissipated, $\eta$. Therefore, energy is transported from large to small scales with a constant transfer rate $\varepsilon>0$ 
in a cascade-like manner (Frisch 1995). A time-reversal transformation, $t \rightarrow-t$, leads to a change of direction of this energy flux, showing that turbulent flows are not symmetric in time.

Since mixing is a good indicator for the irreversibility of a flow, it seems natural to use the dispersion of particle clusters as a tool to analyze time asymmetry. The simplest case is the dispersion of a set of two particles, called relative dispersion (for an overview, see e.g. Sawford 2001, Salazar \& Collins 2009). Many investigations have been conducted concerning this topic, most of them focusing on the mean squared separation forwards in time, $\left\langle\mathbf{R}(t)^{2}\right\rangle$, where $\mathbf{R}(t)$ is the separation vector between the two particles for time $t>0$ and all pairs start with an initial separation $|\mathbf{R}(0)|=R_{0}$. For an initial separation much larger than the dissipative scale, but still smaller than the injection scale, $\eta \ll R_{0} \ll L$, Batchelor (1952) showed that for very short times the mean squared separation grows as

$$
\left\langle\mathbf{R}(t)^{2}\right\rangle \propto\left(\varepsilon R_{0}\right)^{\frac{2}{3}} t^{2}
$$

This expression communicates the idea that particles initially continue their path ballistically according to $\mathbf{x}(t) \approx \mathbf{x}(0)+\mathbf{v}(0) t$. This leads to a quadratic, and thus symmetric, dependence on time. For later times, when $\eta \ll R_{0} \ll|\mathbf{R}(t)| \ll L$, it is expected that the mean squared separation grows according to Richardson scaling (Richardson 1926, Obukhov 1941, Batchelor 1952)

$$
\left\langle\mathbf{R}(t)^{2}\right\rangle=g \varepsilon t^{3},
$$

where $g$ is the Richardson constant. Due to the cubic dependence on time, this scaling law is sensitive to a time asymmetry of the flow and it is assumed that there are two different constants $g_{f}$ and $g_{b}$ for dispersion forwards $(t>0)$ and backwards in time $(t<0)$, respectively (Sawford et al.2005). First experimental and numerical studies on the time asymmetry in relative dispersion indeed indicated that particles separate faster backwards than forwards in time (Sawford et al. 2005, Berg et al. 2006, Bragg et al. 2014). This finding was attributed to Richardson scaling, and attempts were made to obtain values for $g_{f}$ and $g_{b}$. Richardson scaling is notoriously hard to observe, however, and a rigorous, mathematical explanation for the observed enhancement backwards in time is still lacking.

It is thus crucial to find a theoretical, reliable connection between the observed time asymmetry in particle dispersion and the intrinsic time irreversibility of turbulent flows due to the energy cascade. In this thesis, I show that this connection can be found by investigating the evolution of clusters of two and four particles at very short times. I confirm my theoretical findings with experimental data conducted in a turbulent water flow produced by two counterrotating propellers. Additionally, I explore how a change of the energy cascade, induced by the addition of minute amounts of polymers to the flow, is reflected in the particle dispersion. Using experimental data, I confirm the theoretical framework recently proposed by Xi et al. (2013) which suggests that the polymer-flow interactions severely alter the energy cascade as soon as the energy dissipated by the polymers equals the kinetic energy transfer of the turbulence. I then present experimental data showing that the addition of polymers to the flow has a significant impact on time asymmetry in both twoand four-particle dispersion. 
Chapter 1 provides a brief introduction into the field of turbulence, including a more detailed section about multi-particle dispersion forwards and backwards in time. The experimental setup and data-evaluation techniques are explained in chapter 2. Afterwards, the results on two-particle dispersion and four-particle dispersion are given in chapters 3 and 4 , respectively. In chapter 5 , the impact of polymers on the energy cascade is discussed and results for particle dispersion in this scenario are shown. Finally, the presented results are evaluated in chapter 6 and some promising further research possibilities are highlighted.

Parts of this thesis have been published in Physical Review Letters (Jucha et al. 2014). 



\section{CHAPTER 1 \\ Introduction and Theory}

This chapter aims to convey the underlying theoretical concepts and the current state of research in the field of turbulent multi-particle dispersion. Some of these concepts are well known in the turbulence community and are described in detail in e.g. Monin \& Yaglom (2007), Frisch (1995), Argyris et al. (2010), or Pope (2000). Wherever possible, however, I will rely on the excellent and historically important texts by Richardson (1922), Kolmogorov $(1941 a, b)$, and Batchelor (1950) in order to present these ideas.

Section 1.1 describes the governing equations of a turbulent flow and explains the necessity of a statistical description. Section 1.2 focuses on the famous theory of Kolmogorov (Kolmogorov 1941a) and the underlying picture of the turbulence energy cascade. In section 1.3, different aspects of turbulent dispersion for two or more fluid particles are described and current theoretical and experimental findings are presented.

\subsection{The Governing Equations}

The first fundamental assumption when dealing with fluid dynamics is the continuum approximation. This assumption implies that the fluid fills space continuously and that its composition of molecules and ions can be neglected. This approximation is valid as long as the smallest scales of the flow are much larger than the mean free path in the fluid, a condition satisfied by the majority of flows. In a strongly turbulent cumulus cloud, e.g., the smallest scales of the flow are of the order of $\eta \approx 10^{-3} \mathrm{~m}$ and thus some orders of magnitude larger than the typical mean free path ${ }^{1}$. Using the continuum approximation, the equations for fluid motion can be derived from first principles.

\footnotetext{
${ }^{1}$ The smallest turbulent scale is estimated from measurements in atmospheric clouds at an altitude of approx. $2000 \mathrm{~m}$ (Siebert et al. 2006). The atmospheric pressure at this height is about $65 \mathrm{kPa}$ for a sea-surface temperature of $288 \mathrm{~K}$ and an average molecular mass of the atmosphere of $0.02897 \mathrm{~kg} /$ mole, leading to a mean free path of $\lambda \approx 10^{-7} \mathrm{~m}$.
} 


\subsubsection{The Navier-Stokes Equations}

The evolution of a fluid element is governed by two conservation laws: the conservation of mass and the conservation of momentum. Mass conservation leads to the continuity equation

$$
\frac{\partial}{\partial t} \rho(\mathbf{x}, t)+\nabla \cdot(\rho(\mathbf{x}, t) \mathbf{u}(\mathbf{x}, t))=0,
$$

with $\rho(\mathbf{x}, t)$ being the density of the fluid element and $\mathbf{u}(\mathbf{x}, t)$ being its velocity. Momentum conservation, together with the second Newtonian theorem, results in

$$
\frac{\mathrm{d}}{\mathrm{d} t}(\rho(\mathbf{x}, t) \mathbf{u}(\mathbf{x}, t))=\left(\frac{\partial}{\partial t}+\mathbf{u}(\mathbf{x}, t) \cdot \nabla\right)(\rho(\mathbf{x}, t) \mathbf{u}(\mathbf{x}, t))=\nabla \cdot \sigma(\mathbf{x}, t)+\rho(\mathbf{x}, t) \mathbf{f}(\mathbf{x}, t),
$$

with the stress tensor $\sigma(\mathbf{x}, t)$ and $\mathbf{f}(\mathbf{x}, t)$ being the body force (e.g. gravity or the Coriolis force).

Most natural flows exhibit incompressibility, meaning that the density remains constant along fluid trajectories, according to $\frac{\partial}{\partial t} \rho(\mathbf{x}, t)+(\mathbf{u}(\mathbf{x}, t) \cdot \nabla) \rho(\mathbf{x}, t)=0$. In the case of lowintensity water flows, where only very small Mach numbers are reached, it is justified to even assume a constant density, $\rho(\mathbf{x}, t) \equiv \rho$, throughout the flow. For the rest of this thesis, the density is assumed to be constant if not stated otherwise. In this case, the above equations simplify to

$$
\begin{aligned}
\nabla \cdot \mathbf{u}(\mathbf{x}, t) & =0, \\
\rho\left(\frac{\partial}{\partial t}+\mathbf{u}(\mathbf{x}, t) \cdot \nabla\right) \mathbf{u}(\mathbf{x}, t) & =\nabla \cdot \sigma(\mathbf{x}, t)+\rho \mathbf{f}(\mathbf{x}, t) .
\end{aligned}
$$

For an incompressible viscous fluid, the stress tensor is given by

$$
\sigma_{i j}(\mathbf{x}, t)=-p(\mathbf{x}, t) \delta_{i j}+\mu\left(\frac{\partial u_{i}(\mathbf{x}, t)}{\partial x_{j}}+\frac{\partial u_{j}(\mathbf{x}, t)}{\partial x_{i}}\right),
$$

where $p(\mathbf{x}, t)$ is the scalar pressure field, $\mu$ is the dynamic viscosity, and $\delta_{i j}$ is the Kronecker delta. With this stress tensor and equation (1.4), one obtains the Navier-Stokes equations (Navier 1827, Stokes 1845) for a fluid with constant density:

$$
\rho\left(\frac{\partial}{\partial t} \mathbf{u}(\mathbf{x}, t)+(\mathbf{u}(\mathbf{x}, t) \cdot \nabla) \mathbf{u}(\mathbf{x}, t)\right)=-\nabla p(\mathbf{x}, t)+\mu \triangle \mathbf{u}(\mathbf{x}, t)+\rho \mathbf{f}(\mathbf{x}, t) .
$$

Together with the continuity equation (1.3) and initial as well as boundary conditions, the Navier-Stokes equations fully describe a fluid flow. It is informative to non-dimensionalize equation (1.6) by introducing the new variables

$$
\mathbf{x}^{\prime}=\mathbf{x} / L, \quad \mathbf{u}^{\prime}=\mathbf{u} / U, \quad t^{\prime}=t U / L, \quad p^{\prime}=p /\left(\rho U^{2}\right) \quad \text { and } \quad \mathbf{f}^{\prime}=\mathbf{f} L / U^{2},
$$

with $L$ and $U$ being the characteristic length scale and velocity of the flow. Inserting the new 
variables into equation (1.6), dividing by $U^{2} / L$ and omitting the primes, one obtains

$$
\frac{\partial}{\partial t} \mathbf{u}(\mathbf{x}, t)+(\mathbf{u}(\mathbf{x}, t) \cdot \nabla) \mathbf{u}(\mathbf{x}, t)=-\nabla p(\mathbf{x}, t)+\frac{1}{\operatorname{Re}} \triangle \mathbf{u}(\mathbf{x}, t)+\mathbf{f}(\mathbf{x}, t) .
$$

The only free parameter in equation (1.8) is the Reynolds number, $\operatorname{Re}=\frac{U L}{v}$, with the kinematic viscosity $v=\mu / \rho$ (Reynolds 1883 ). For very small Reynolds numbers, $\operatorname{Re} \lesssim 1$, the viscous term is dominating and the flow is purely laminar, i.e. all stream lines are in parallel. For $\operatorname{Re} \gtrsim 10^{3}$, on the other hand, the viscous damping term is very weak and turbulence can evolve (Reynolds 1883, Avila et al. 2011). For turbulent, atmospheric flows one can find Reynolds numbers as high as $\operatorname{Re} \approx 10^{8}-10^{9}$.

The Navier-Stokes equations are nonlinear and, due to the pressure term, also non-local. For turbulent flows, for which the damping term, $\frac{1}{\operatorname{Re}} \triangle \mathbf{u}(\mathbf{x}, t)$, becomes relatively weal due to a high Reynolds number, this leads to a phenomenon called deterministic chaos: Tiny differences in the initial conditions lead to very different outcomes, making the flow unpredictable (see e.g. Lorenz 1963). Only averaged quantities can be reproduced reliably, showing the necessity of a statistical approach to turbulence.

\subsubsection{Statistical Description of Turbulence}

A natural first attempt for a statistical description of turbulent flows is the averaging of the Navier-Stokes equations in order to obtain an expression for the mean flow. This was first done by Reynolds (1895) using a time average. In this section, I will use an ensemble average instead, but the concept remains the same.

Let us assume several independent statistical events, e.g. repetitions of the same experiment, where for each event a random variable $x$ is measured. The ensemble average of $x$ is then defined as

$$
\langle x\rangle=\lim _{N \rightarrow \infty} \frac{1}{N} \sum_{n=1}^{N} x_{n}
$$

with $x_{n}$ being the values of $x$ for the different events. Due to the chaotic behavior of turbulent flow, the velocity field $\mathbf{u}(\mathbf{x}, t)$ can be taken as such a random variable, more precisely a random field. Using the ensemble average, one can split the velocity field into a mean value and a fluctuating component in the form

$$
\mathbf{u}(\mathbf{x}, t)=\overline{\mathbf{u}}(\mathbf{x}, t)+\mathbf{u}^{\prime}(\mathbf{x}, t)
$$

with $\overline{\mathbf{u}}(\mathbf{x}, t)=\langle\mathbf{u}(\mathbf{x}, t)\rangle$. From this definition, it directly follows that $\langle\overline{\mathbf{u}}(\mathbf{x}, t)\rangle=\overline{\mathbf{u}}(\mathbf{x}, t)$ and $\left\langle\mathbf{u}^{\prime}(\mathbf{x}, t)\right\rangle=0$. Inserting the above decomposition into the continuity and Navier-Stokes equations (1.3) and (1.8) and taking the ensemble average leads to

$$
\begin{aligned}
\nabla \cdot \overline{\mathbf{u}}(\mathbf{x}, t) & =0, \\
\frac{\partial}{\partial t} \overline{\mathbf{u}}(\mathbf{x}, t)+(\overline{\mathbf{u}}(\mathbf{x}, t) \cdot \nabla) \overline{\mathbf{u}}(\mathbf{x}, t)+\left\langle\left(\mathbf{u}^{\prime}(\mathbf{x}, t) \cdot \nabla\right) \mathbf{u}^{\prime}(\mathbf{x}, t)\right\rangle & =-\nabla\langle p(\mathbf{x}, t)\rangle+\frac{1}{\operatorname{Re}} \triangle \overline{\mathbf{u}}(\mathbf{x}, t)+\langle\mathbf{f}(\mathbf{x}, t)\rangle .
\end{aligned}
$$

This new set of four equations comprises seven variables. Aside from the mean flow variables 
$\overline{\mathbf{u}}(\mathbf{x}, t)$ and $\langle p(\mathbf{x}, t)\rangle$, the last term on the left hand side depends quadratically on the velocity fluctuations $\mathbf{u}^{\prime}(\mathbf{x}, t)$. It is usually written in the form

$$
\left\langle\left(\mathbf{u}^{\prime}(\mathbf{x}, t) \cdot \nabla\right) \mathbf{u}^{\prime}(\mathbf{x}, t)\right\rangle_{i}=\sum_{j} \frac{\partial}{\partial x_{j}}\left\langle u_{j}^{\prime}(\mathbf{x}, t) u_{i}^{\prime}(\mathbf{x}, t)\right\rangle,
$$

where the components of $\left\langle u_{j}^{\prime}(\mathbf{x}, t) u_{i}^{\prime}(\mathbf{x}, t)\right\rangle$ are known as the Reynolds stresses. Determining the mean velocity $\overline{\mathbf{u}}(\mathbf{x}, t)$ thus requires the knowledge of the second moment of the velocity fluctuations, meaning that the equations for $\overline{\mathbf{u}}(\mathbf{x}, t)$ are not closed. This closure problem prevents a statistical description of turbulence solely based on the Navier-Stokes equations (Kraichnan 1961), and some modeling is required in order to formulate results (for an overview, see e.g. Pope 2000, chap. 10-14).

\subsection{Kolmogorov's Theory (1941)}

The works published by Kolmogorov in 1941 are amongst the most important achievements in turbulence research (Frisch 1995). He built the foundation of today's understanding of turbulent flow and provided one of the few exact results derived from the Navier-Stokes equations.

Kolmogorov refined the picture of the turbulence energy cascade, first promoted by Richardson (1922), and used it as a foundation for his work (Kolmogorov 1941a). According to this picture, energy injected into a three-dimensional (3D) turbulent flow will form eddies ${ }^{2}$ of some large scale $L$ (e.g. the size of a propeller stirring the fluid). These eddies break up into smaller eddies, thereby transferring their energy to smaller scales with an energy transfer rate $\varepsilon$. This process continues until a length scale $\eta$ is reached at which viscous dissipation dominates (figure 1.1). Kolmogorov further assumed that a statistical decoupling happens during this energy transfer. As a consequence, eddies much smaller than the injection scale $L$ should be unaffected by the nature of the injection. From this he concluded his first hypothesis of similarity:

\section{Statistical quantities depending only on the smallest scales of the flow are fully determined by the energy transfer rate $\varepsilon$ and the kinetic viscosity $v .^{3}$}

It follows directly that these quantities need to be homogeneous and isotropic because any preference of orientation of the largest eddies is lost at the small scales ${ }^{4}$. Using only $\varepsilon$ and $v$, one is also able to construct universal time, length, and velocity scales, the Kolmogorov microscales:

$$
\eta=\left(\frac{v^{3}}{\varepsilon}\right)^{\frac{1}{4}}, \quad \tau_{\eta}=\left(\frac{v}{\varepsilon}\right)^{\frac{1}{2}}, \quad u_{\eta}=(v \varepsilon)^{\frac{1}{4}}
$$

\footnotetext{
${ }^{2}$ An eddy is to be understood as "a component of motion with a certain length scale, i.e. an arbitrary flow pattern characterized by size alone" (Batchelor 1950). It is not to be confused with a whirl as seen near a drain. ${ }^{3}$ Due to the mathematical nature of the original hypotheses in (Kolmogorov 1941a), a more verbose formulation is chosen here.

${ }^{4}$ The concept of isotropic turbulence and its usefulness to compute exact relations had already been presented earlier in the important works of Taylor (1935) and Von Kármán \& Howarth (1938).
} 


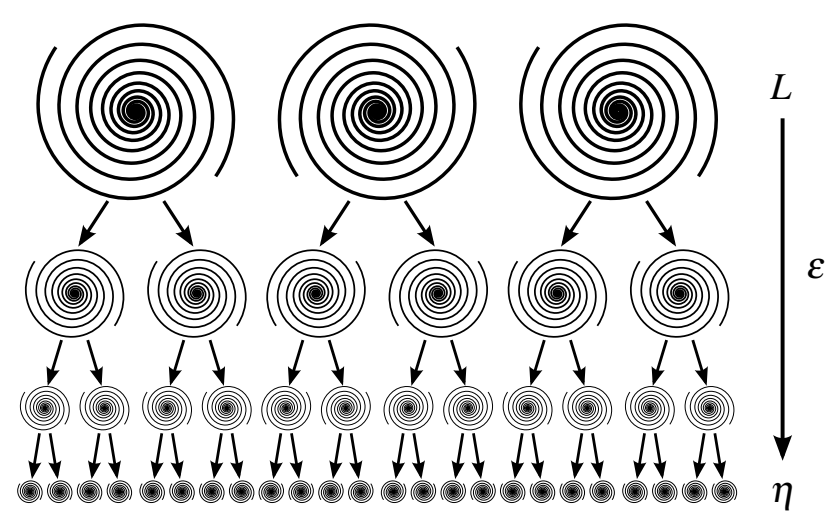

Figure 1.1.: Sketch of the energy cascade as used by Kolmogorov (1941a).

By construction, these are the scales corresponding to the smallest eddies of the flow with a Reynolds number of $\operatorname{Re}_{\eta}=\frac{u_{\eta} \eta}{v}=1$. While $\eta$ represents the size of the eddies, $\tau_{\eta}$ gives the eddy turn-over time and $u_{\eta}$ the resulting characteristic velocity. Changes of the flow along distances much smaller than $\eta$ or during time intervals much shorter than $\tau_{\eta}$ are assumed to be smooth and differentiable. The scale ratio between the large energy containing eddies and the small dissipative eddies is known to grow as $L / \eta \propto \operatorname{Re}^{\frac{3}{4}}$ and $T_{L} / \tau_{\eta} \propto \operatorname{Re}^{\frac{1}{2}}$ with $T_{L}$ being the eddy turn-over time of the large eddies. For very strong turbulence, and thus very high Reynolds number, the scale separation $L / \eta$ becomes very large. In this case, Kolmogorov suggested that there exists a range of scales, $\eta \ll r \ll L$, which is unaffected both by the large scales and by dissipation. This leads to his second hypothesis of similarity:

\section{For very large Reynolds numbers, there exists an intermediate range of scales for which any statistical quantity is fully determined by the scale $r$ and the energy transfer rate $\varepsilon$.}

This intermediate range of scales, the inertial range, is therefore independent of the underlying conditions of the flow field (i.e. forcing, geometry, and viscosity) and should yield the same statistics for all flows with the same energy transfer rate. This conclusion is of major importance to the entire field of turbulence research, since it allows the comparison of results obtained from different flows.

Starting from these two hypotheses, one can use dimensional analysis to obtain some useful results for the limit of $\operatorname{Re} \rightarrow \infty$. For the hypotheses to be applicable, however, the statistical quantities under consideration need to be assignable to a certain scale. A good choice for such a quantity are the longitudinal structure functions,

$$
S_{p}(r)=\left\langle\left(\frac{(\mathbf{u}(\mathbf{x}+\mathbf{r}, t)-\mathbf{u}(\mathbf{x}, t)) \cdot \mathbf{r}}{r}\right)^{p}\right\rangle,
$$

with $p$ being a positive integer. Due to homogeneity, the structure functions depend only on the separation vector $\mathbf{r}$, while isotropy can be used to further simplify this dependence to 
the absolute value $r=|\mathbf{r}|$. Using the second hypothesis and dimensional analysis, it can be shown that

$$
S_{p}(r) \propto(\varepsilon r)^{\frac{p}{3}} .
$$

For the special case of $p=3$, Kolmogorov even derived an exact result from the NavierStokes equations (Kolmogorov 1941b). Only assuming homogeneity and isotropy, he showed that in the limit of infinite Reynolds number, the third order structure function behaves as

$$
S_{3}(r)=-\frac{4}{5} \varepsilon r,
$$

where $r$ is in the inertial range. This 4/5-law is one of the few exact results in turbulence research and therefore plays an important role in the description of turbulence ${ }^{5}$.

It was later shown that an alternative formulation to the 4/5-law can be obtained by looking at the derivative of the squared relative velocity

$$
\frac{1}{2}\left\langle\frac{\mathrm{d}}{\mathrm{d} t}[\mathbf{u}(\mathbf{x}+\mathbf{r}, t)-\mathbf{u}(\mathbf{x}, t)]^{2}\right\rangle=\langle[\mathbf{u}(\mathbf{x}+\mathbf{r}, t)-\mathbf{u}(\mathbf{x}, t)] \cdot[\mathbf{a}(\mathbf{x}+\mathbf{r}, t)-\mathbf{a}(\mathbf{x}, t)]\rangle=-2 \varepsilon .
$$

This result is exact for a homogeneous flow at a high Reynolds number and with $\mathbf{r}$ in the inertial range (Mann et al. 1999, Falkovich et al. 2001, Pumir et al. 2001). Since I will use equation (1.16) frequently in this thesis, I will from now on use the short notation $\langle\delta \mathbf{u} \cdot \delta \mathbf{a}\rangle=-2 \varepsilon$ whenever referring to this expression.

\subsection{Turbulent Dispersion}

Another area of turbulence research in which the theory of Kolmogorov can be put to great use is turbulent dispersion. The separation statistics of clusters of $n$ particles in a turbulent flow are of crucial importance for the understanding of many fundamental processes, like the spreading of clouds or turbulent mixing (Sawford 2001, Ottino 1989a). Furthermore, as opposed to single particle diffusion, the characteristic length scale of a cluster of particles dominates the statistics thereof so that Kolmogorov's theory can be applied (Batchelor 1950). The easiest case, the separation of pairs, of course plays a special role in theoretical, experimental, and numerical investigations (Sawford 2001, Salazar \& Collins 2009).

\subsubsection{Eulerian and Lagrangian Descriptions of Turbulence}

Studying particle separation requires to follow particle trajectories through a flow. This is best done by the so-called Lagrangian decription of turbulence (Yeung 2002, Toschi \& Bodenschatz 2009). As opposed to the Eulerian framework in which the flow field is given at fixed points in space and time, the Lagrangian framework describes flow properties along the trajectories of fluid particles. Here, I use the term fluid particle, or fluid element, to describe a single point in the fluid continuum which follows the velocity field. It is an abstract

\footnotetext{
${ }^{5}$ It is known for many years that the scaling law in equation (1.14) is actually not exact for $p \neq 3$ due to intermittency effects. For a longer discussion of this topic see e.g. (Frisch 1995, chap. 8).
} 
mathematical concept, not to be confused with e.g. a molecule. If such a fluid particle starts at a position $\mathbf{y}$ at time $t=0$, then its position at a later time $t$ will be denoted by $\mathbf{X}(t \mid \mathbf{y}, 0)$ with $\mathbf{X}(0 \mid \mathbf{y}, 0)=\mathbf{y}$. By construction, the velocity of a fluid particle is always identical to the velocity field at its position, that is, $\frac{\mathrm{d}}{\mathrm{d} t} \mathbf{X}(t \mid \mathbf{y}, 0)=\mathbf{U}(t \mid \mathbf{y}, 0)=\mathbf{u}(\mathbf{X}(t \mid \mathbf{y}, 0), t)$. Experimentally, tracer particles with the same density as the fluid and a diameter $d \ll \eta$ much smaller than the Kolmogorov scale can be treated as fluid particles.

\subsubsection{Pair Dispersion}

The displacement statistics of a pair of fluid particles can be described by the probability density function (p.d.f.) $P\left(\mathbf{x}_{1}, \mathbf{x}_{2}, t \mid \mathbf{y}_{1}, \mathbf{y}_{2}, 0\right)$ of finding the particles at positions $\mathbf{x}_{1}$ and $\mathbf{x}_{2}$ at time $t$ under the condition that they have been at positions $\mathbf{y}_{1}$ and $\mathbf{y}_{2}$ at time $t=0$ (Batchelor 1952). In the Lagrangian notation introduced above, this means that $\mathbf{X}\left(t \mid \mathbf{y}_{1}, 0\right)=\mathbf{x}_{1}$ and $\mathbf{X}\left(t \mid \mathbf{y}_{2}, 0\right)=\mathbf{x}_{2}$. Separating the relative positioning $\mathbf{R}=\mathbf{x}_{2}-\mathbf{x}_{1}$ and $\mathbf{R}_{0}=\mathbf{y}_{2}-\mathbf{y}_{1}$ from the general movement $\mathbf{M}=\mathbf{x}_{1}-\mathbf{y}_{1}$, we obtain the more useful p.d.f. $P\left(\mathbf{M}, \mathbf{R}, t \mid \mathbf{R}_{0}, 0\right)$, where the dependence on the initial positions $\mathbf{y}_{1}$ and $\mathbf{y}_{2}$ is omitted under the assumption of a homogeneous flow. In many cases, as for example the growth of a particle cloud, the collective motion of the two particles is of no interest, and the separation p.d.f.

$$
P_{r}\left(\mathbf{R}, t \mid \mathbf{R}_{0}, 0\right)=\int P\left(\mathbf{M}, \mathbf{R}, t \mid \mathbf{R}_{0}, 0\right) \mathrm{d} \mathbf{M}
$$

provides an adequate description. For an isotropic homogeneous flow, the first moment of this p.d.f. vanishes and the second moment is given by

$$
\left\langle\left[\mathbf{X}\left(t \mid \mathbf{y}_{1}, 0\right)-\mathbf{X}\left(t \mid \mathbf{y}_{2}, 0\right)\right]^{2}\right\rangle \equiv\left\langle\mathbf{R}(t)^{2}\right\rangle=\int \mathbf{R}^{2} P_{r}\left(\mathbf{R}, t \mid \mathbf{R}_{0}, 0\right) \mathrm{d} \mathbf{R} .
$$

This second moment, the mean squared separation, gives the relative dispersion of the two fluid particles and its evolution can be written in the very general form

$$
\frac{\mathrm{d}}{\mathrm{d} t}\left\langle\mathbf{R}(t)^{2}\right\rangle=F\left(R_{0}, t\right)
$$

where, due to the assumption of homogeneity and isotropy, the function $F$ can only depend on the absolute value $R_{0}=\left|\mathbf{R}_{0}\right|$ of the initial separation. With this, Kolmogorov's hypotheses can be applied to learn more about the function $F$ and I will loosely follow Batchelor (1950) for this procedure.

As a prerequisite for the first similarity hypothesis, $|\mathbf{R}(t)|$ needs to be much smaller than the energy injection scale $L$, which results in the requirement that $t$ and $R_{0}$ be "not too large." Under these conditions, equation (1.19) can be expressed more precisely as

$$
\frac{\mathrm{d}}{\mathrm{d} t}\left\langle\mathbf{R}(t)^{2}\right\rangle=v F\left(\frac{R_{0} \varepsilon^{\frac{1}{4}}}{v^{\frac{3}{4}}}, \frac{t \varepsilon^{\frac{1}{2}}}{v^{\frac{1}{2}}}\right),
$$

where the arguments of $\mathrm{F}$ are now dimensionless. In the case where $R_{0}$, and thus $|\mathbf{R}(t)|$, is in 
the inertial range, the second similarity hypothesis states that $F$ can only depend on $\varepsilon$ and the involved time and spatial scales. This leads to

$$
\frac{\mathrm{d}}{\mathrm{d} t}\left\langle\mathbf{R}(t)^{2}\right\rangle=\varepsilon t^{2} F\left(\frac{R_{0}}{\varepsilon^{\frac{1}{2}} t^{\frac{3}{2}}}\right),
$$

where the only constructible dimensionless variable depends on both $R_{0}$ and $t$. Aside from restricting $R_{0}$, we can of course also constrain the time variable:

very small $\mathbf{t}$ : The mean squared separation can always be rewritten in terms of the particle velocities as

$$
\frac{\mathrm{d}}{\mathrm{d} t}\left\langle\mathbf{R}(t)^{2}\right\rangle=2\left\langle\left(\mathbf{R}(0)+\int_{0}^{t} \mathbf{V}\left(t^{\prime}\right) \mathrm{d} t^{\prime}\right) \cdot \mathbf{V}(t)\right\rangle
$$

with $\mathbf{V}(t)=\mathbf{U}\left(t \mid \mathbf{y}_{1}, 0\right)-\mathbf{U}\left(t \mid \mathbf{y}_{2}, 0\right)$ being the relative velocity between the two particles. In his original work, Batchelor (1950) assumed the initial separation and the velocity to be uncorrelated, so that $\langle\mathbf{R}(0) \cdot \mathbf{V}(t)\rangle$ can be set to zero. Furthermore, in the limit where $t$ is very small, one finds that $\mathbf{V}\left(t^{\prime}\right) \approx \mathbf{V}(t) \approx \mathbf{V}(0)$ within the limits of the integration. This should be valid as long as the two velocities are still strongly correlated, meaning that $t \ll t_{0}$, where $t_{0}=\left(R_{0}^{2} / \varepsilon\right)^{\frac{1}{3}}$ is the characteristic Kolmogorov time for an eddy of size $R_{0}{ }^{6}$. For $R_{0}$ in the inertial range, equation (1.21) then reduces to

$$
\frac{\mathrm{d}}{\mathrm{d} t}\left\langle\mathbf{R}(t)^{2}\right\rangle \approx 2 t\left\langle\mathbf{V}(0)^{2}\right\rangle=2 \frac{11}{3} C_{2}\left(\varepsilon R_{0}\right)^{\frac{2}{3}} t .
$$

This scaling law for the particle separation is known as Batchelor scaling. Note that the prescribed linearity in time as a result of $t$ being small, together with the assumption of $R_{0}$ being in the inertial range, are sufficient to completely determine $F$ up to an unknown constant $C_{2}$.

intermediate t: For a sufficiently high Reynolds number and $R_{0}$ in the inertial range, there is a range of intermediate times, $t>t_{0}$, for which $|\mathbf{R}(t)|$ is still in the inertial range but much larger than the initial separation. In this case, one can argue that $\frac{\mathrm{d}}{\mathrm{d} t}\left\langle\mathbf{R}(t)^{2}\right\rangle$ does not depend on the exact value of $R_{0}$, so that equation (1.21) simplifies to

$$
\frac{\mathrm{d}}{\mathrm{d} t}\left\langle\mathbf{R}(t)^{2}\right\rangle \approx 3 g \varepsilon t^{2}
$$

Again, the evolution of the pair separation is fully determined except for a constant $g$, termed Richardson constant. Equation (1.24) is respectively known as the RichardsonObukhov law (Richardson 1926, Obukhov 1941).

Apart from these early findings, another more recent consideration shall be presented here. As mentioned above, Batchelor (1950) assumed the initial separation and relative velocity to

\footnotetext{
${ }^{6}$ One can also think of $t_{0}$ as the $e d d y$-turn-over time of an eddy of size $R_{0}$. After one turn-over, the eddy looses its coherence and the velocities of two fluid particles belonging to the eddy become uncorrelated.
} 
be uncorrelated. Without this assumption, equation (1.23) changes to

$$
\begin{aligned}
\frac{\mathrm{d}}{\mathrm{d} t}\left\langle\mathbf{R}(t)^{2}\right\rangle & \approx 2\langle\mathbf{R}(0) \cdot \mathbf{V}(0)\rangle+2 t\left\langle\mathbf{V}(0)^{2}\right\rangle \\
& \approx 2\langle\mathbf{R}(0) \cdot \mathbf{V}(0)\rangle+C_{2}\left(\varepsilon R_{0}\right)^{\frac{2}{3}} t,
\end{aligned}
$$

which can be re-expressed in the form of the mean squared change of separation

$$
\frac{\mathrm{d}}{\mathrm{d} t}\left\langle\delta \mathbf{R}^{2}(t)\right\rangle \equiv \frac{\mathrm{d}}{\mathrm{d} t}\left\langle[\mathbf{R}(t)-\mathbf{R}(0)]^{2}\right\rangle \approx C_{2}\left(\varepsilon R_{0}\right)^{\frac{2}{3}} t .
$$

For a perfectly homogeneous and isotropic flow, equation (1.24) and equation (1.26) are identical. There is some evidence, however, that for real flows, both experimentally and numerically, this condition is only approximately fulfilled. In this case, the mean squared change of separation shows much clearer scaling than the original expression by Batchelor and should be used instead (Ouellette et al. 2006b). The Richardson-Obukhov law, on the other hand, is identical for both the mean squared separation and the mean squared change of separation since an independence from the initial separation was assumed in the derivation.

Please note that in the Lagrangian notation, the relation for $\langle\delta \mathbf{u} \cdot \delta \mathbf{a}\rangle$ in equation (1.16) can be conveniently written as

$$
\frac{1}{2}\left\langle\left.\frac{\mathrm{d}}{\mathrm{d} t} \mathbf{V}(t)^{2}\right|_{t=0}\right\rangle=\langle\mathbf{V}(0) \cdot \mathbf{A}(0)\rangle=-2 \varepsilon
$$

where $\mathbf{A}(t)=\frac{\mathrm{d}}{\mathrm{d} t} \mathbf{V}(t)$ is the relative acceleration between the two particles. This expression can be understood as the Lagrangian equivalent of the 4/5-law.

\subsubsection{Backward Dispersion}

I have thus far focused on the dispersion of particle pairs over time that start with some fixed initial separation. Naming this process forward dispersion, one can distinguish another concept, where a fixed separation is prescribed at a later time and the dispersion of particle pairs prior to that time is analyzed. This is called backward dispersion. As shown above, the forward dispersion of a pair of fluid particles is given by $\left\langle\mathbf{R}(t)^{2}\right\rangle=\int \mathbf{R}^{2} P_{r}\left(\mathbf{R}, t \mid \mathbf{R}_{0}, 0\right) \mathrm{d} \mathbf{R}$, with $P_{r}\left(\mathbf{R}, t \mid \mathbf{R}_{0}, 0\right)$ being the probability that $\mathbf{X}\left(t \mid \mathbf{y}_{2}, 0\right)-\mathbf{X}\left(t \mid \mathbf{y}_{1}, 0\right)=\mathbf{R}$ for $\mathbf{y}_{2}-\mathbf{y}_{1}=\mathbf{R}_{0}$. Accordingly, the backward dispersion can be written as

$$
\left\langle\mathbf{R}(-t)^{2}\right\rangle=\int \mathbf{R}^{2} P_{r}\left(\mathbf{R},-t \mid \mathbf{R}_{0}, 0\right) \mathrm{d} \mathbf{R}=\int \mathbf{R}^{2} P_{r}\left(\mathbf{R}_{0}, t \mid \mathbf{R}, 0\right) \mathrm{d} \mathbf{R},
$$

where stationarity and incompressibility ${ }^{7}$ of the flow are used for the second equality. The alternative formulations in equation (1.28) correspond to the two notions that the particle pair is either tracked from its initial condition backwards in time towards an earlier state, or

\footnotetext{
${ }^{7}$ For an incompressible flow, it can be shown that the displacement probability of two particles obeys $P\left(\mathbf{x}_{1}, \mathbf{x}_{2}, t \mid \mathbf{y}_{1}, \mathbf{y}_{2}, 0\right)=P\left(\mathbf{y}_{1}, \mathbf{y}_{2}, 0 \mid \mathbf{x}_{1}, \mathbf{x}_{2}, t\right)$ (Lundgren 1981), which directly leads to $P_{r}\left(\mathbf{R}, t \mid \mathbf{R}_{0}, 0\right)=$ $P_{r}\left(\mathbf{R}_{0}, 0 \mid \mathbf{R}, t\right)$.
} 


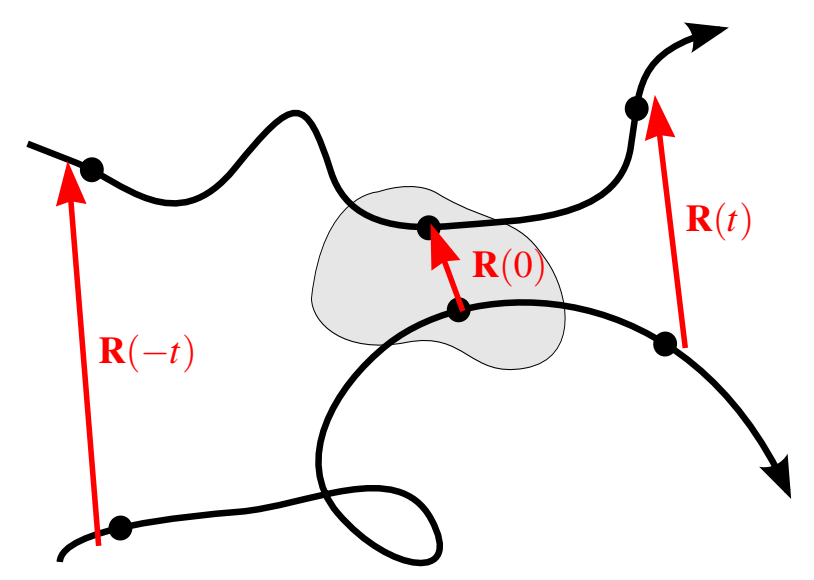

Figure 1.2.: Sketch of the relative motion of two fluid particles with a fixed separation $|\mathbf{R}(0)|=R_{0}$ at $t=0$. If the particles are located inside a cloud of material at $t=0$, forward dispersion captures the dispersion of the cloud with time while backward dispersion is connected to the concentration field at $t=0$.

from an earlier state towards a fixed end state. Both interpretations are equally useful and show an important difference to forward dispersion as illustrated in figure 1.2. Assuming that the fluid particles are part of a material cloud at $t=0$, forward dispersion describes the spreading of the cloud whereas backward dispersion describes its formation. The latter provides valuable information about how advected material coming from different locations of a flow is brought together, which is apparently closely connected to scalar concentration fields and turbulent mixing (Corrsin 1952, Durbin 1980, Frisch et al. 1999, Thomson 2003, Celani et al. 2004, Sawford et al. 2005).

While forward dispersion has been studied extensively since the early works of Taylor (1922) and Richardson (1926), backward dispersion has only recently become focus of turbulence research. For the relative dispersion of fluid particles with an initial separation $R_{0}$ in the inertial range, recent studies found that dispersion happens faster backwards in time than forwards (Sawford et al. 2005, Berg et al. 2006, Bragg et al. 2014). Sawford et al. (2005) proposed that this time asymmetry can be captured by extending Richardson scaling towards the backward case with

$$
\mathbf{R}^{2}(t)= \begin{cases}R_{0}^{2}+g_{f} \varepsilon t^{3} & \text { for } t>0 \\ R_{0}^{2}+g_{b} \varepsilon|t|^{3} & \text { for } t<0\end{cases}
$$

where the two scaling constants have been experimentally measured to be $g_{f}=0.55$ and $g_{b}=1.15$ (Berg et al. 2006). The reliability of these result will be discussed later in this thesis. 


\subsubsection{Multi-Particle Dispersion}

In recent years, new experimental techniques and ever-growing computer clusters have enabled us to go beyond pair statistics and take first steps in the direction of multi-particle dispersion (Chertkov et al. 1999, Falkovich et al. 2001, Biferale et al. 2005, Lüthi et al. 2007, Hackl et al. 2011, Xu et al. 2011, Pumir et al. 2013). This overview will be restricted to the theoretical essentials for describing $n$-particle dispersion with a focus on $n=4$. Further developments and ideas will be covered in chapter 4 as well as the discussion section of this thesis.

The major benefit of looking at clusters of $n>2$ fluid particles is that beyond the size of the cluster, there is a multitude of additional shape information. In order to capture all of this information, a clever description of the cluster is needed. In a homogeneous, $d$-dimensional flow, a cloud of $n$ particles can be described by a set of $n-1$ position vectors. In the field of turbulence research, the usual notation for this set of vectors is

$$
\left.\rho^{(m)}(t)=\sqrt{\frac{m}{m+1}}\left[\mathbf{X}\left(t \mid \mathbf{y}^{(m+1)}, 0\right)-\frac{1}{m} \sum_{l=1}^{m} \mathbf{X}\left(t, \mathbf{y}^{(l)}, 0\right)\right)\right], \quad m \in\{1,2, \ldots, n-1\},
$$

with $\rho^{(m)}$ being the $m$ th $\rho$-vector. One can now construct a $d \times(n-1)$ matrix

$$
\mathrm{P}(t)=\left(\rho^{(1)}(t) \rho^{(2)}(t) \ldots \rho^{(n-1)}(t)\right),
$$

which contains the full geometrical information of the cluster. The singular value decomposition of $\mathrm{P}(t)$ is then given by

$$
\mathrm{P}=\mathbf{U}(t) \operatorname{diag}\left(\sigma_{1}(t), \sigma_{2}(t), \ldots, \sigma_{\min (d, n-1)}(T)\right) \mathbf{W}^{T}(t) .
$$

Here, $\mathbf{W}(t)$ is an $(n-1) \times(n-1)$ orthogonal matrix and gives the orientation in pseudospace, a vector space spanned by the numbering of the $\rho$-vectors. The singular values, $\sigma_{i}$, contain the elongation of the cluster along different axes, and the $d \times d$ orthogonal matrix $\mathbf{U}(t)$ gives the orientation in real space. In an isotropic flow, the orientation of the cluster in real space does not matter, so that the full cluster can be described by $\min (d, n)$ singular values and $(n-1)(n-2) / 2$ Euler angles in pseudo-space. Since P does not transform as a tensor, however, it is helpful to define the moment of inertia or shape tensor,

$$
\mathbf{G}(t)_{i j}=\sum_{a=1}^{n} \rho_{i}^{(a)}(t) \rho_{j}^{(a)}(t)=\left(\mathrm{P}(t) \mathrm{P}^{T}(t)\right)_{i j}
$$

with $i, j \in\{1,2, \ldots, d\}$ being spatial indices ${ }^{8}$. The eigenvalues $g_{i}(t)$ of the shape tensor are given by the squares of the singular values of $\mathrm{P}(t)$ and thus must be real and non-negative. With this definition, the shape of any cluster of $n$ particles can be fully described by the

\footnotetext{
${ }^{8}$ Instead of the shape tensor, one can also define the dispersion tensor $\mathbf{C}(t)=\mathrm{P}^{T}(t) \mathrm{P}(t)=$ $\mathbf{W}(t) \operatorname{diag}\left(\sigma_{1}^{2}, \sigma_{2}^{2}, \ldots, \sigma_{\min (d, n-1)}^{2}\right) \mathbf{W}^{T}(t)$. It has the same eigenvalues as $\mathbf{G}(t)$ and serves the same purpose (Hackl et al. 2011).
} 
eigenvalues of the shape tensor together with $(n-1)(n-2) / 2$ Euler angles. It is helpful to sort the eigenvalues by size, with $g_{1}(t) \geq g_{2}(t) \geq \ldots \geq g_{n-1}(t)$.

The size of the cluster can be described by the radius of gyration, which is defined as the squared distances of the individual particles from the center of mass of the cluster, $\mathbf{X}_{\mathrm{com}}(t)$. In terms of the shape tensor, the radius of gyration is simply given by

$$
R^{2}(t)=\sum_{m}\left(\mathbf{X}\left(t \mid \mathbf{y}^{(m)}, 0\right)-\mathbf{X}_{\mathrm{com}}(t)\right)^{2}=\operatorname{tr}(G(t))=\sum_{i} g_{i}(t) .
$$

\section{Four-Particle Clusters}

A cluster of four particles in a 3D flow is of particular interest. Four points, forming a tetrahedron, is the minimum constellation needed to describe a 3D volume. The number of needed $\rho$-vectors to span the tetrahedron is given by $n-1=3$ and equals the dimension of the flow. The $\rho$ - vectors are given by

$$
\begin{aligned}
& \rho^{(1)}(t)=\sqrt{\frac{1}{2}}\left[\mathbf{X}\left(t \mid \mathbf{y}^{(2)}, 0\right)-\mathbf{X}\left(t \mid \mathbf{y}^{(1)}, 0\right)\right], \\
& \rho^{(2)}(t)=\sqrt{\frac{2}{3}}\left[\mathbf{X}\left(t \mid \mathbf{y}^{(3)}, 0\right)-\frac{1}{2} \mathbf{X}\left(t \mid \mathbf{y}^{(1)}, 0\right)-\frac{1}{2} \mathbf{X}\left(t \mid \mathbf{y}^{(2)}, 0\right)\right], \\
& \rho^{(3)}(t)=\sqrt{\frac{3}{4}}\left[\mathbf{X}\left(t \mid \mathbf{y}^{(4)}, 0\right)-\frac{1}{3} \mathbf{X}\left(t \mid \mathbf{y}^{(1)}, 0\right)-\frac{1}{3} \mathbf{X}\left(t \mid \mathbf{y}^{(2)}, 0\right)-\frac{1}{3} \mathbf{X}\left(t \mid \mathbf{y}^{(3)}, 0\right)\right] .
\end{aligned}
$$

Figure 1.3 shows the special configuration of a regular tetrahedron, together with the corresponding $\rho$-vectors. For a regular tetrahedron, all edges have the same length $l$ and the $\rho$-vectors are perpendicular to each other with $\rho^{(n)} \cdot \rho^{(m)}=\frac{l^{2}}{2} \delta_{n m}$, where $\delta_{n m}$ is the Kronecker delta.

Due to $(n-1)=d=3$, one finds that there are exactly three eigenvalues $g_{1} \geq g_{2} \geq g_{3}$ and three Euler angles needed to fully describe the tetrahedron. The eigenvalues alone suffice for some classification:

- For $g_{1}=g_{2}=g_{3}$ the tetrahedron is regular.

- For $g_{1} \approx g_{2} \gg g_{3}$ the tetrahedron is close to two-dimensional (2D) ("pancake-like").

- For $g_{1} \gg g_{2} \approx g_{3}$ the tetrahedron is close to one-dimensional (1D) ("needle-like").

Recently, it was found that tetrahedra starting with a regular shape with edge length $l \ll L$ at $t=0$ deform into elongated pancake-like shapes with $g_{1}>g_{2} \gg g_{3}$ at later times (Pumir et al. 2000, Biferale et al. 2005, Xu et al. 2008). This deformation can be explained by the concept of the perceived velocity gradient tensor (Chertkov et al. 1999). In analogy to the usual velocity gradient tensor, $A_{i j}(\mathbf{x}, t)=\frac{\partial u_{i}(\mathbf{x}, t)}{\partial x_{j}}$, the perceived velocity gradient tensor, $M(t)$, is defined over the four points of a tetrahedron by

$$
\mathbf{v}^{(a)}(t)=\rho^{(a)}(t)^{T} M(t),
$$




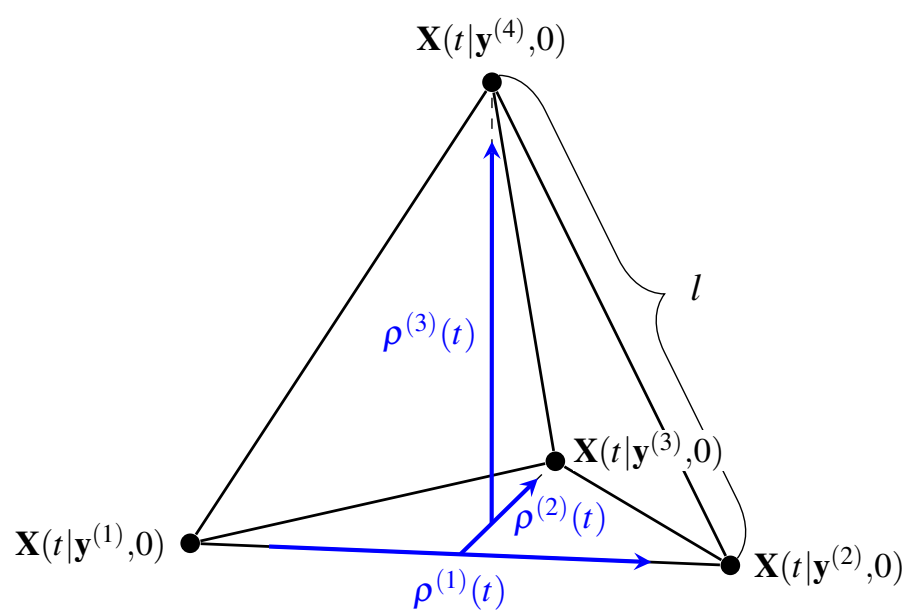

Figure 1.3.: A regular tetrahedron formed by a cluster of four fluid particles. All edges have the same length, $l$. Three $\rho$-vectors are needed to fully describe the tetrahedron in a homogeneous flow.

with $\mathbf{v}^{(a)}(t)=\frac{\mathrm{d}}{\mathrm{d} t} \rho^{(a)}(t)$ being the change in the $\rho$-vectors. From this, a perceived rate of strain and perceived rate of rotation tensor can be derived as

$$
S(t)=\frac{1}{2}\left[M(t)+M(t)^{T}\right] \quad \text { and } \quad \Omega(t)=\frac{1}{2}\left[M(t)-M(t)^{T}\right] .
$$

For $l$ in the dissipative range, these quantities are identical to the usual rate of strain and rate of rotation tensors, $S_{A}(\mathbf{x}, t)$ and $\Omega_{A}(\mathbf{x}, t)$, respectively. For an incompressible flow, one can show that the velocity gradient tensor, and thus also the rate of strain tensor, are traceless, that is $\operatorname{tr}(A(t))=\operatorname{tr}\left(S_{A}(t)\right)=0$. This leads to the fact that the largest eigenvalue of $S_{A}(t)$, $s_{1}$, is positive and the smallest one, $s_{3}$, is negative. Most importantly, however, Betchov (1956) showed that, on average, the intermediate eigenvalue $\left\langle s_{2}\right\rangle$ is positive as well. As a consequence, there exist two straining directions and one compressing direction for most of the time, leading to a coplanar transformation of a fluid element. Even though this result was derived for the dissipative range, it was also confirmed both experimentally and numerically for the perceived rate of strain tensor, $S(t)$, with a somewhat smaller but still positive value of $\left\langle s_{2}\right\rangle$ (Lüthi et al. 2007, Xu et al. 2008, Pumir et al. 2013). Consequently, also for tetrahedra in the inertial range, a deformation into co-planar structures is expected.

Aside from the qualitative results for the shape deformation, a universal behavior of the shape eigenvalues was observed. If time is rescaled with $\tau_{\eta}$, for $R_{0}$ in the dissipative range, or $t_{0}$, for $R_{0}$ in the inertial range, the obtained results for various values of initial separation and Reynolds number collapse onto one curve (Pumir et al. 2000, Xu et al. 2008). Here, $t_{0}=\left(l^{2} / \varepsilon\right)^{\frac{1}{3}}$ is again the characteristic time for an eddy of size $l$. Furthermore, in analogy to pair dispersion, a Richardson $t^{3}$ scaling is expected for the eigenvalues $g_{i}$ and for the radius of gyration $\left\langle R^{2}(t)\right\rangle$ at times $t \gg \tau_{\eta}$. So far, however, this scaling behavior was not observed unambiguously (Pumir et al. 2000, Biferale et al. 2005, Xu et al. 2008). 



\section{CHAPTER 2}

\section{Experimental Methods}

All experimental datasets used in this thesis were obtained by optical Lagrangian Particle Tracking (LPT) ${ }^{1}$. In this measurement technique, a fluid flow is seeded with tracer particles, whose motion is recorded from at least two angles. From the obtained videos, the trajectories of the tracer particles in 3D space are then reconstructed (Maas et al. 1993, Malik et al. 1993). This reconstruction requires accurate, fast, and noise-robust algorithms, which are explained in detail in section 2.1. Once the particles have been tracked, the velocity and acceleration of the flow are computed along each trajectory. This step requires some care, and the procedure is explained in section 2.2.

The experimental setup considered in this thesis consists of a turbulent water flow produced by two counter-rotating propellers. As tracer particles, I used polystyrene microspheres which were illuminated by a strong laser and then recorded by three high-speed cameras. This setup, as well as the experimental procedure to record the videos, is described in detail in section 2.3. Section 2.4 presents the datasets used in this thesis by means of their recording parameters and some derived quantities (e.g. the Reynolds number) and section 2.5 covers the topic of measurement uncertainties. All further data processing will be discussed in the results part of this work.

\subsection{Lagrangian Particle Tracking}

Over many decades, Eulerian measurement techniques have provided a host of information on turbulent flows. The most widely used technique is surely hot-wire anemometry, in which the flow speed of a fluid is obtained at a single point in space by measuring the amount of heat that is carried away from a small heated wire (King 1914). More suited for the

\footnotetext{
${ }^{1}$ Another often-used term is Particle Tracking Velocimetry (PTV). Historically, however, this term was used for a technique measuring a Eulerian flow field (Adrian 1991), so that LPT should be used in order to avoid ambiguities.
} 
analysis of flow patterns is particle image velocimetry (PIV). In the standard implementation of this technique, a 2D area of a densely seeded flow is illuminated by a laser sheet, and two pictures are taken in fast succession. These pictures are then divided into subareas, and the correlation between the corresponding subareas of both images is computed. This can be used to reconstruct the flow field at one instance in time (Adrian 1991).

It is apparent that, despite the importance of these techniques for understanding turbulent flow, they do not provide a full picture. Especially for intrinsically Lagrangian phenomena like turbulent mixing or dispersion, studying the trajectories of fluid particles moving with the flow can provide great additional insight. Due to this, Lagrangian measurement techniques like LPT were developed. While optical LPT systems have been employed for more than 50 years (Chiu \& Rib 1956), widespread use only started in the early 1990's, when high-speed cameras and digital image processing allowed for high temporal and spatial resolution and automated data processing.

Indeed, the right choice of data processing algorithms for reconstructing the particle trajectories is at the core of LPT measurements and crucial to its accuracy. The trajectory reconstruction can be split into three steps:

- Finding the particle centers on the image plane of each camera.

- Matching the particle centers from all cameras to reconstruct 3D particle positions.

- Tracking the particles in time.

In the next sections, I will describe each of these steps in detail and explain which algorithms were used for analyzing the recorded data. All three steps are implemented in a C++ particle-tracking code ${ }^{2}$. I loosely follow Ouellette et al. (2006a).

\subsubsection{Particle Center Finding}

In order to successfully reconstruct as many particle trajectories as possible, it is most crucial to locate the particles on the camera image planes with high precision. The search algorithm must thus identify the particle centers with subpixel accuracy at non-vanishing noise levels and with low computational costs. Furthermore, overlapping particles must be distinguished.

The scattered light of a particle that is illuminated by a bright light source will be represented by a certain intensity profile on each of the camera image planes. Figure 2.1 shows an image recorded by one of the high-speed cameras, as well as two close-up views. If particles are close to each other or behind each other from the perspective of one of the cameras, the intensity profiles will overlap and build a cluster of bright pixels with several local maxima on the image plane of this camera. I will use the common assumption that each intensity maximum corresponds to one particle.

For the case of particles with an arbitrary intensity profile, a simple, weighted average can be used to obtain the particle centers. To do this, however, pixel clusters with more than one intensity maximum need to be separated into subgroups in order to distinguish

\footnotetext{
${ }^{2}$ I would like to acknowledge that all $\mathrm{C}++$ codes for the LPT procedure presented in this section were written by Nicholas T. Ouellette and Haitao Xu (Ouellette et al. 2006a, Xu 2008).
} 


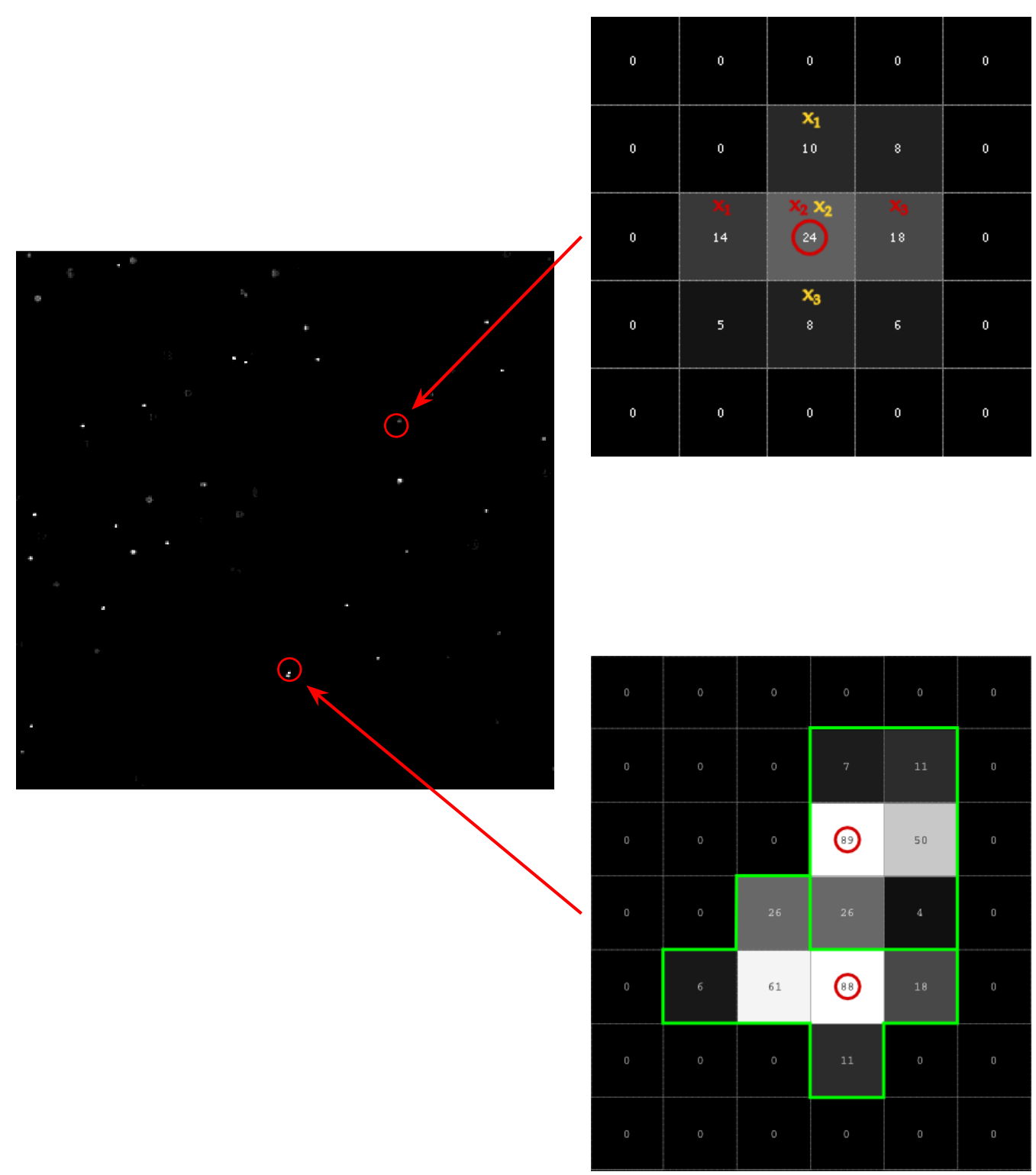

Figure 2.1.: A subsection of $320 \times 320$ pixels from a frame of a recorded video. The image displays approximately an area of $(5 \mathrm{~cm})^{2}$. For better visibility, the color map is rescaled to the lowest 64 gray values. The original video has $640 \times 640$ pixels and 256 gray values ( 8 bit). Close-up views of the intensity profile for a single particle (top) and for two overlapping particles (bottom) are shown on the right. Each pixel is labeled with its gray value and red circles indicate the intensity maxima. For each of the pictures on the right, one center finding method is sketched exemplarily. For the single particle profile, the pixels used for the Gaussian fit method are labeled in red (horizontal) and yellow (vertical). For the two overlapping particle profiles, the edges of the pixel subgroups as used for the weighted average method are shown in green. 
the overlapping particle intensity profiles. Following Maas et al. (1993), this devision can be accomplished by assuming that the light intensity of a particle profile should decrease monotonically. The pixels with the lowest intensity are assigned to the neighboring pixel with the highest intensity (see figure 2.1). For every subgroup, corresponding each to one particle, a weighted average can be applied. With $\sum_{p}$ being the sum over all pixels of a subgroup around an intensity maximum, the particle center is given by

$$
\mathbf{x}_{c}=\frac{\sum_{p} \mathbf{x}_{p} I\left(\mathbf{x}_{p}\right)}{\sum_{p} I\left(\mathbf{x}_{p}\right)}
$$

This center-finding method is very fast and has an acceptable robustness to noise, but it comes at the cost of poor resolution (Ouellette et al. 2006a).

If a functional expression for the particle intensity profile were known, a corresponding fit would of course yield a much better result for the particle center. Despite the lack of such an exact functional expression, it was shown that the intensity profile of a spherical particle can be approximated well by a 2D Gaussian function (Westerweel 1993, section 2.5). Since a Gaussian profile has only one intensity maximum, fitting 2D Gaussian profiles to each maximum of a group of bright pixels already distinguishes overlapping particles, and a separate step to split the cluster into subgroups is not needed. A 2D Gaussian fit, however, is computationally expensive and needs a high number of involved pixels to produce accurate results. A minimum of five pixels are needed to determine all fit parameters. It was shown by Ouellette et al. (2006a) that two 1D Gaussian functions, one for the horizontal and another for the vertical particle position, yield similar or better results for a noisy image (see also Cowen \& Monismith 1997). Additionally, when limiting the fits to the intensity maximum and its closest horizontal and vertical neighbors, an analytical solution can be found. Fitting the intensity profile

$$
I\left(x_{i}\right)=\frac{I_{0}}{\sqrt{2 \pi} \sigma} \exp \left[-\frac{1}{2}\left(\frac{x_{i}-x_{c}}{\sigma}\right)^{2}\right]
$$

to the intensities of the pixels at positions $x_{i} \in\left\{x_{1}, x_{2}, x_{3}\right\}$ (see figure 2.1), one obtains for the particle center in this direction

$$
x_{c}=\frac{1}{2} \frac{\left(x_{1}^{2}-x_{2}^{2}\right) \ln \left[I\left(x_{2}\right) / I\left(x_{3}\right)\right]-\left(x_{2}^{2}-x_{3}^{2}\right) \ln \left[I\left(x_{1}\right) / I\left(x_{2}\right)\right]}{\left(x_{1}-x_{2}\right) \ln \left[I\left(x_{2}\right) / I\left(x_{3}\right)\right]-\left(x_{2}-x_{3}\right) \ln \left[I\left(x_{1}\right) / I\left(x_{2}\right)\right]} .
$$

Since the intensities $I\left(x_{i}\right)$ are digitized, e.g. with $I\left(x_{i}\right) \in\{0, . ., 255\}$ for an 8-bit image, the logarithms in equation (2.3) can be precomputed once and reused as often as needed during the image processing. Fitting the intensity profile with two 1D Gaussian functions thus leads to a very efficient image analysis with excellent results. Therefore, this center finding method was used for all datasets presented in this thesis. 


\subsubsection{Calibration}

In order to reconstruct the 3D coordinates of the particles from their center points on the image planes, it is necessary to know where a certain point in the measurement volume would appear on the different camera image planes. For this purpose, a relation between the world (or laboratory) coordinates of a point and its image coordinates needs to be established. This can be accomplished by executing a calibration procedure before each series of recordings. For the work presented in this thesis, a calibration method developed by Tsai (1987) was used which shall be briefly explained here. The procedure can be divided into four steps.

\section{Step 1: From world coordinates to camera coordinates}

The world coordinate of a point $P$ in the measurement volume shall be given by $\mathbf{x}_{w}=\left(x_{w}, y_{w}, z_{w}\right)^{T}$, where the origin, $\mathcal{O}_{w}$, and the orientation of the world reference frame can be arbitrarily chosen. One now defines a new 3D coordinate system, whose origin $\mathcal{O}_{c}$ is at the optical center of the camera and whose $x y$-plane is parallel to the camera image plane. In this camera reference frame, the coordinates of the point $P$ are given by

$$
\mathbf{x}_{c}=\mathbf{R} \cdot \mathbf{x}_{w}+\mathbf{T},
$$

where $\mathbf{R}$ is a rotation matrix and $\mathbf{T}=\left(T_{x}, T_{y}, T_{z}\right)^{T}$ a translation vector. This transformation requires the knowledge of six external parameters, three Euler angles for $\mathbf{R}$ and the three components of $T$, in order to describe the arrangement of the camera system.

\section{Step 2: Undistorted projection onto the image plane}

Assuming an ideal pinhole camera with an effective focal length $f$, the projection of the point $P$ onto the image plane yields

$$
\left(\begin{array}{l}
x_{u} \\
y_{u}
\end{array}\right)=\frac{f}{z_{c}}\left(\begin{array}{l}
x_{c} \\
y_{c}
\end{array}\right)
$$

with $x_{u}$ and $y_{u}$ being the undistorted image coordinates. The effective focal length $f$ is given by the distance between the image plane and the optical center $\mathcal{O}_{c}$.

\section{Step 3: Adding distortion}

In addition to the projection in step 2, every real camera system introduces some level of distortion. The resulting distorted image plane coordinates can be written as

$$
\left(\begin{array}{l}
x_{d} \\
y_{d}
\end{array}\right)+\left(\begin{array}{l}
D_{x} \\
D_{y}
\end{array}\right)=\left(\begin{array}{l}
x_{u} \\
y_{u}
\end{array}\right)
$$

with some general distortion functions $D_{x}\left(\mathbf{x}_{d}\right)$ and $D_{y}\left(\mathbf{x}_{d}\right)$. According to Tsai (1987), the full camera distortion can be well approximated by the leading order term of a purely radial distortion, so that $D_{x} \approx \kappa r^{2} x_{d}$ and $D_{y} \approx \kappa r^{2} y_{d}$ with $r^{2}=x_{d}^{2}+y_{d}^{2}$. The distorted image plane coordinates are thus given by

$$
\left(\begin{array}{l}
x_{d} \\
y_{d}
\end{array}\right)\left(1+\kappa r^{2}\right)=\left(\begin{array}{l}
x_{u} \\
y_{u}
\end{array}\right)=\frac{f}{z_{c}}\left(\begin{array}{l}
x_{c} \\
y_{c}
\end{array}\right)
$$


where equation (2.5) was used at the second equality. Equation (2.7) contains two intrinsic parameters, the effective focal length, $f$, and the distortion parameter, $\kappa$.

\section{Step 4: From image coordinates to computer coordinates}

The last step deals with the transformation from the image plane coordinates to the computer image coordinates as stored by the camera. Allowing different spacings between the pixels in the horizontal and vertical direction, the final computer coordinates are given by

$$
\left(\begin{array}{l}
x_{f} \\
y_{f}
\end{array}\right)=\left(\begin{array}{c}
s_{x} x_{d} \\
y_{d}
\end{array}\right)
$$

For an ideal pixel array, the parameter $s_{x}$ should equal unity. In this case, $s_{x}$ can be used later as a measure for the accuracy of the calibration. Note that Tsai (1987) introduces several more parameters for the transformation in equation (2.8), some of which can be absorbed into the effective focal length while some others do not apply to the cameras used here.

Joining steps one through four, the relation between the world coordinates of point $P$ and its coordinates $\left(x_{f}, y_{f}\right)$ on the recorded computer image is given by

$$
\begin{aligned}
\frac{1}{s_{x}}\left(1+\kappa r^{2}\right) x_{f} & =f \frac{\mathbf{R}_{x} \cdot \mathbf{x}_{w}+T_{x}}{\mathbf{R}_{z} \cdot \mathbf{x}_{w}+T_{z}}, \\
\left(1+\kappa r^{2}\right) y_{f} & =f \frac{\mathbf{R}_{y} \cdot \mathbf{x}_{w}+T_{y}}{\mathbf{R}_{z} \cdot \mathbf{x}_{w}+T_{z}}, \\
\text { with } \quad r^{2} & =\left(\frac{1}{s} x_{f}\right)^{2}+y_{f}^{2},
\end{aligned}
$$

using the notation $\mathbf{R}=\left(\mathbf{R}_{x}, \mathbf{R}_{y}, \mathbf{R}_{z}\right)^{T}$. Tsai (1987) pointed out that the lack of tangential distortion, as given by step 3 , leads to great simplifications in solving the above equations. Since a pure radial distortion does not change the direction of the vector $\mathbf{x}_{u}$ on the image plane and, by construction, $\left(x_{u}, y_{u}\right)$ has the same orientation as $\left(x_{c}, y_{c}\right)$, one finds that $\mathbf{x}_{d}$ and $\left(x_{c}, y_{c}\right)$ must be parallel. This leads to the mathematical requirement of a vanishing cross product, $\mathbf{x}_{d} \times \mathbf{x}_{c}=x_{d} y_{c}-y_{d} x_{c}=0$, called the radial alignment constraint. In terms of the world and computer coordinates, this cross product can be rewritten as

$$
\frac{1}{s_{x}} x_{f}\left(\mathbf{R}_{y} \cdot \mathbf{x}_{w}+T_{y}\right)-y_{f}\left(\mathbf{R}_{x} \cdot \mathbf{x}_{w}+T_{x}\right)=0 .
$$

With the correct choice of variables, this expression can be cast into a set of linear equations with

$$
\left(-x_{d} \mathbf{x}_{w}, y_{d} \mathbf{x}_{w}, y_{d}\right) \cdot\left(\begin{array}{c}
\mathbf{R}_{y} / T_{y} \\
s_{x} \mathbf{R}_{x} / T_{x} \\
s_{x} T_{x} / T_{y}
\end{array}\right)=x_{d} .
$$

For known world and computer coordinates of enough points $P$, the full rotation matrix, as well as the parameters $T_{x}, T_{y}$, and $s_{x}$, can be determined. The remaining parameters, $T_{z}, f$, and $\kappa$, can then be obtained by iteratively solving equations (2.9) and (2.10). 


\subsubsection{D Matching}

After a relation between laboratory and image coordinates has been established, the matching procedure is straightforward (see figure 2.2). For each particle center found on the image plane of camera 1, the line of possible positions in the measurement volume is calculated. With some tolerance band, this line is then projected onto all other cameras. For each particle center within this band, this particle's line of possible positions is then calculated as well. The minimum distance for all possible combinations of particle positions is calculated and the best hit is considered as the real particle position.

Please note that with this method ambiguities can arise from particles that, in the coordinate system of one of the cameras, are right behind each other and are thus projected onto the same point on the image plane of this camera. These ambiguities arise especially for high particle-seeding densities, and they can only be resolved by using more than two cameras (usually three or four). Furthermore, Maas et al. (1993) pointed out that more cameras also lead to a better accuracy of the particle coordinates.

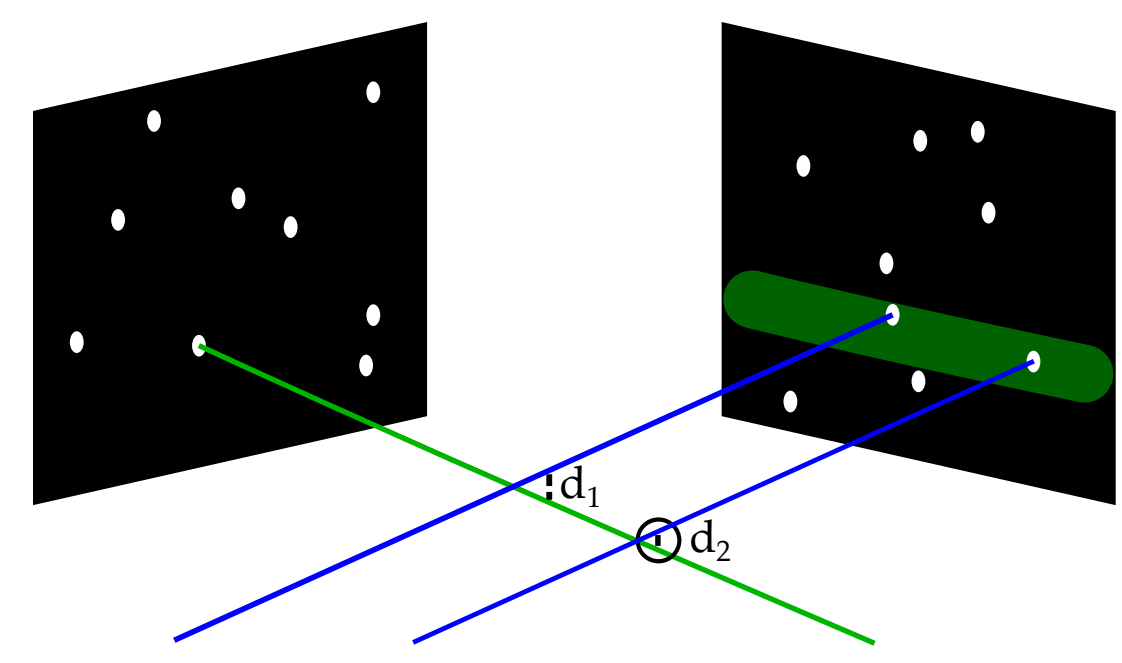

Figure 2.2.: Reconstruction of the 3D particle coordinates from the particle centers on the image planes of two cameras. The green line is the line of possible positions for one particle center on the left image plane, which is then projected onto the right image plane (green band). For each particle center in the band, a line of possible positions is constructed as well (blue lines). The "real particle position" is chosen to be that combination of possible particle positions from both image planes for which the distance $d_{i}$ is smallest (circle). 


\subsubsection{Particle Tracking}

There are multiple algorithms for tracking particles in time, all with different advantages and disadvantages. In Ouellette et al. (2006a), several of these algorithms were compared, and a four-frame, predictive algorithm showed the best performance for a numerically-simulated turbulent flow. This method is implemented in the particle-tracking code used for this thesis and shall be explained briefly.

The first two time steps of a particle trajectory are linked together by the nearest neighbor method (see figure 2.3(a)). Then, for any time step $n$ with particle coordinates $\mathbf{x}_{n}$, the position of the particle at time step $n+1$ is estimated according to $\tilde{\mathbf{x}}_{n+1}=\mathbf{x}_{n}+\tilde{\mathbf{v}}_{n} \Delta t$, where $\tilde{\mathbf{v}}_{n}$ is a velocity computed from the frames $n$ and $n-1$, and $\Delta t$ is the time lapse between two frames. For each particle occurrence in the vicinity of this hypothetical position, a particle position at time step $n+2$ is then estimated as $\tilde{\mathbf{x}}_{n+2}=\mathbf{x}_{n}+\tilde{\mathbf{v}}_{n}(2 \Delta t)+\frac{1}{2} \tilde{\mathbf{a}}_{n}(2 \Delta t)^{2}$, where $\tilde{\mathbf{a}}_{n}$ is an acceleration computed from the frames $n-1$ to $n+1$. For the continuation of a track, the particle position at time step $n+1$ for which the prediction at $n+2$ is closest to a measured particle position is picked (see figure 2.3(b)). The other considered positions are rejected and the algorithm proceeds with the next time step. This algorithm can handle occasional loss of the particle image for up to three frames.

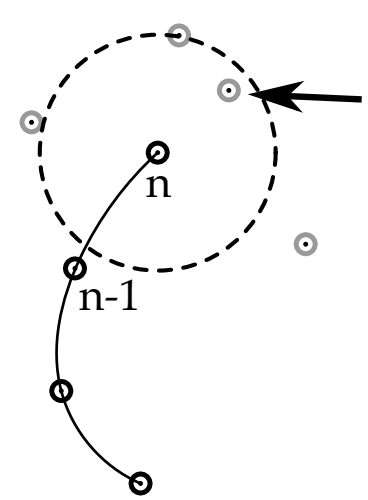

(a) Nearest Neighbor

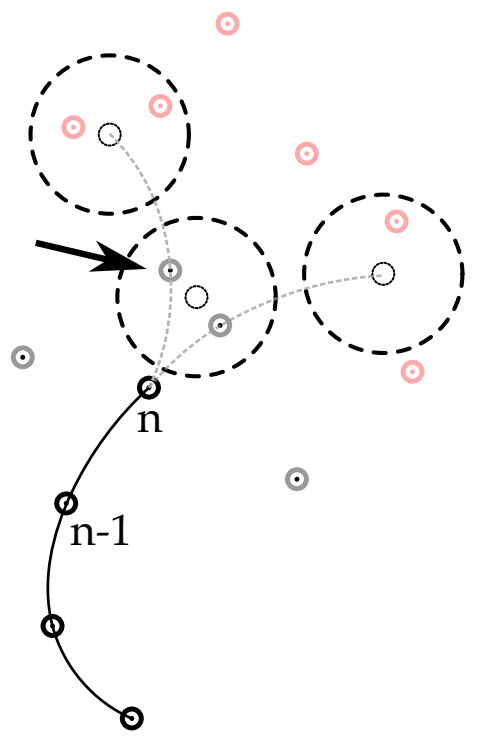

(b) Four-Frame Best Estimate

Figure 2.3.: Sketch of the used tracking algorithm. Black circles and black lines represent already connected particle positions. Gray circles indicate possible particle positions at time step $n+1$, while red circles indicate possible particle positions at time step $n+2$. Open black circles indicate estimated particle positions and large dashed circles represent the corresponding search area. The arrows indicate the chosen particle positions at time step $n+1$. Adapted from Ouellette (2006) with friendly permission. 


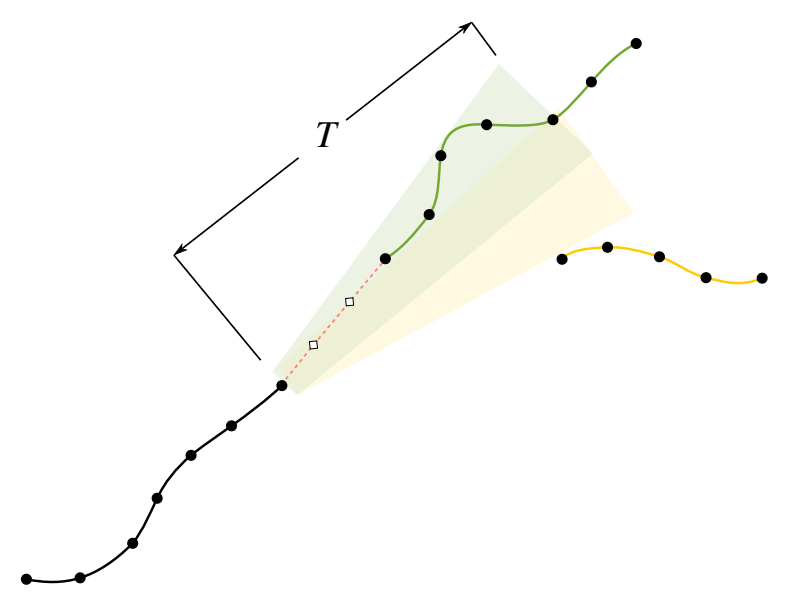

Figure 2.4.: Sketch of the reconnection of a broken trajectory. A broken trajectory (black line) and two hypothetical continuations (green and yellow lines) are shown. Each dot on those lines represents one particle position. For each hypothetical continuation, it is checked if the distance in position-velocity space is smaller than a certain search radius (sketched as green and yellow cones). From the continuations fulfilling this condition - here only the green one - the continuation with the shortest distance is chosen. The missing points between the broken trajectory and its continuation are spanned by linear interpolation (red dashed line).

If an ambiguity appears during the tracking process, the track is stopped at the current frame and a new track is started at the next frame. The same happens if a particle is lost for more than three frames due to insufficient illumination. Some of these broken tracks can be reconnected after the completion of the main tracking process. In this thesis, an algorithm similar to the one described by Xu (2008) is used. For each trajectory, the position and velocity for the first and last frame are used to reconnect tracks in position-velocity space. Starting with a trajectory $i$, for which the position and velocity at the end point $t_{i}^{e}$ are given by $\mathbf{x}_{i}^{e}$ and $\mathbf{u}_{i}^{e}$, respectively, each other trajectory $j$ with a starting point $t_{j}^{s}$ and $0<t_{j}^{s}-t_{i}^{e}<T$ is taken as a hypothetical continuation. The chosen maximum search time $T$ must be smaller than the time in which the average distance traveled by a particle equals the average separation between the particles in the flow. For each hypothetical continuation $j$, the trajectory $i$ is extrapolated linearly for times $t_{i}^{e}<t<t_{j}^{s}$. The extrapolated position and velocity at time $t$ are given by $\mathbf{x}_{i}^{p}(t)=\mathbf{x}_{i}^{e}+\frac{1}{2}\left(\mathbf{u}_{i}^{e}+\mathbf{u}_{j}^{s}\right)\left(t-t_{i}^{e}\right)$ and $\mathbf{u}_{i}^{p}(t)=$ $\mathbf{u}_{i}^{e}$, respectively ${ }^{3}$. The distance in position-velocity space at time $t_{j}^{s}$ is then computed with $d_{i j}=\sqrt{\left|\mathbf{x}_{i}^{p}\left(t_{j}^{s}\right)-\mathbf{x}_{j}^{s}\right|^{2}+\left[\left|\mathbf{u}_{i}^{p}\left(t_{j}\right)-\mathbf{u}_{j}^{s}\right|\left(t_{j}^{s}-t_{i}^{e}\right)\right]^{2}}$. From all hypothetical continuations which fulfill the condition $d_{i j}<d_{m}(t)$, the one with the smallest distance $d_{i j}$ is chosen (see

${ }^{3} \mathrm{Xu}$ (2008) showed that a more advanced computation method of $\mathbf{u}_{i}^{p}(t)$ using the acceleration at time $t_{i}^{e}$ slightly improves the reconnection procedure if the accelerations are well resolved. 
figure 2.4). Here, the search radius grows with time as $d_{m}(t)=\delta x+\frac{d_{\max }-\delta x}{T}\left(t-t_{i}^{e}\right)$, where the search radius at $t=t_{i}^{e}, \delta x$, is given by the uncertainty of the position measurement. If two hypothetical continuations yield the same distance in position-velocity space, the one with an earlier starting point $t_{j}^{s}$ is picked. Once a continuation is chosen, the missing particle positions between the initial trajectory and its continuation are filled by linear interpolation. The interpolated points are marked accordingly in the data file.

All trajectories, reconnected or not, are stored in a specific binary data format, with each file containing all trajectories from one video (see appendix C.2).

\subsection{Velocity and Acceleration Computation}

After the completion of the tracking procedure, the particle velocities and accelerations can be computed along the trajectories. For ideal tracer particles, these coincide with the flow velocity and acceleration at the particle position. Since the particle tracks are only known at discrete time steps (i.e. every frame), there are several options to obtain differentiated quantities: Approximating the derivatives by finite differences; fitting the trajectory and differentiating the resulting function; or using a convolution with a specified kernel. While finite difference methods are very sensitive to noise, Voth et al. (2002) showed that parabolic fitting shows good results, provided that the correct length scale for the fit is chosen. The same holds true for the Gaussian convolution method, which is used in this thesis (Mordant et al. 2004).

For a Lebesgue integrable function $f(s) \in L^{1}(\mathcal{R})$, the convolution with a Gaussian kernel $g(s)=\frac{1}{\sigma \sqrt{2 \pi}} \exp \left[-\frac{1}{2}\left(\frac{s}{\sigma}\right)^{2}\right]$ is given by

$$
(f * g)(s) \equiv \int_{-\infty}^{\infty} f(s-\tau) g(\tau) \mathrm{d} \tau
$$

This convolution smooths out the function $f(s)$ by averaging over all values of $f(s+\tau)$, where the weighting decreases with $|\tau|$. For the derivative of the convoluted function, it can be shown that

$$
(f * g)^{\prime}=f^{\prime} * g=f * g^{\prime},
$$

so that taking the derivative of $f(s)$ and subsequently performing a simple smoothing is equivalent to convoluting $f(s)$ with a differentiated kernel.

For a discrete function $f[n]$ with $n \in \mathbb{N}$, the convolution is defined as

$$
(f * g)[n] \equiv \sum_{m=-M}^{M} f[n-m] g[m],
$$

where $g[m]$ now represents a discretized convolution kernel with a finite support $m \in$ $\{-M,-M+1, \ldots, M\}$ and the normalization condition $\sum_{m} g[m]=1$. In those circumstances where no functional expression of $f[n]$ is known, the second term in equation (2.15) can be used to obtain the derivative of $f[n]$ for a known kernel $g[n]$. For this purpose, the functional expression of the convolution kernel needs to be differentiated, discretized over a finite 
support, and then correctly normalized. For a Gaussian kernel $g(s)$, discretization leads to

$$
g[n]=\frac{1}{\sum_{n} \exp \left[-\left(\frac{n}{w}\right)^{2}\right]} \exp \left[-\left(\frac{n}{w}\right)^{2}\right] \quad \text { for } n \in\left\{-\frac{l}{2},-\frac{l}{2}+1, \ldots, \frac{l}{2}\right\},
$$

where $w$ is the filter width, corresponding to $\sqrt{2} \sigma$, and $l$ is the length of the support, henceforth referred to as filter length. A usual choice for the filter length is $l=3 w$, corresponding to about $4.2 \sigma$. The prefactor in equation (2.17) ensures that the kernel is properly normalized, with $(1 * g[n])=1$. The corresponding derivatives are then given by

$$
\begin{aligned}
g^{\prime}[n] & =\frac{n}{\sum_{n}-n^{2} \exp \left[-\left(\frac{n}{w}\right)^{2}\right]} \exp \left[-\left(\frac{n}{w}\right)^{2}\right] \\
\text { and } g^{\prime \prime}[n] & =\frac{1}{\sum_{n} \frac{n^{2}}{2} g_{0}^{\prime \prime}[n]-\frac{1}{l} \sum_{n} g_{0}^{\prime \prime}[n] \sum_{n} \frac{n^{2}}{2}}\left(g_{0}^{\prime \prime}[n]-\frac{1}{l} \sum_{n} g_{0}^{\prime \prime}[n]\right),
\end{aligned}
$$

with $g_{0}^{\prime \prime}[n]=\left[1-2\left(\frac{n}{w}\right)^{2}\right] \exp \left[-\left(\frac{n}{w}\right)^{2}\right]$. The first derivative is normalized such that $\left(n * g^{\prime}\right)=1$ and $\left(1 * g^{\prime}\right)=0$, while $\left(\frac{n^{2}}{2} * g^{\prime \prime}\right)=1$ and $\left(1 * g^{\prime \prime}\right)=0$ for the second derivative.

Figure 2.5 (a) shows a discretized Gaussian kernel, as well as its first and second derivative, in comparison with the continuous case. An application to a noisy test function is shown in figure 2.5 (b) and illustrates an overall good result. Only the extrema are slightly underestimated, which is to be expected from a smoothing kernel. Figure 2.5 (b) also reveals that, by construction, the derivatives cannot be computed for the last $l / 2$ points at each end of the dataset due to the length of the kernel.

The next important step is to find the best filter length for the convolution kernel. It can be easily deduced from figure 2.5 (b) that for an overly short filter length, in this case e.g. $l=3$, noise has a dominant contribution to the derivatives. On the other hand, if the filter length is too long, e.g. $l=30$, finer variations of the data are lost to the smoothing. Analyzing the root mean square (rms) acceleration for a turbulent flow as a function of the temporal filter length $\tau_{f}=l \delta t$, where $\delta t$ is the time between two frames, Voth et al. (2002) found that no range of $\tau_{f}$ exists for which the result is independent of $\tau_{f}$ (see figure 2.6). They showed, however, that an approximate exponential behavior can be observed for large $\tau_{f}$, while a $\tau_{f}^{-5 / 2}$ scaling due to uncorrelated Gaussian noise is dominant at small $\tau_{f}$. Even though there is no filter length for which the noise contribution is zero and no information is lost to smoothing, there exists a crossover of the two effects where the contribution due to noise becomes small and the loss of information is still acceptable. This is where $\tau_{f}$ is chosen, the exact value being worked out individually for each dataset. All trajectories of all videos of one measurement are then stored in a single binary file, the velacc-file, containing the full information about position, velocity and acceleration of the tracer particles (see appendix C.3).

Once the particle velocity and acceleration have been computed for all trajectories, some Eulerian flow properties are derived, e.g. the velocity and acceleration moments as well as the structure functions $S_{p}(r)$. Note that in the present case of a homogeneous, stationary flow, all relative quantities are statistically independent of position, $\mathbf{x}$, and time, $t$, so that the average can be taken over all particle pairs which have a separation $|\mathbf{R}|=R_{0} \pm \Delta R_{0}$ at any point in time. 

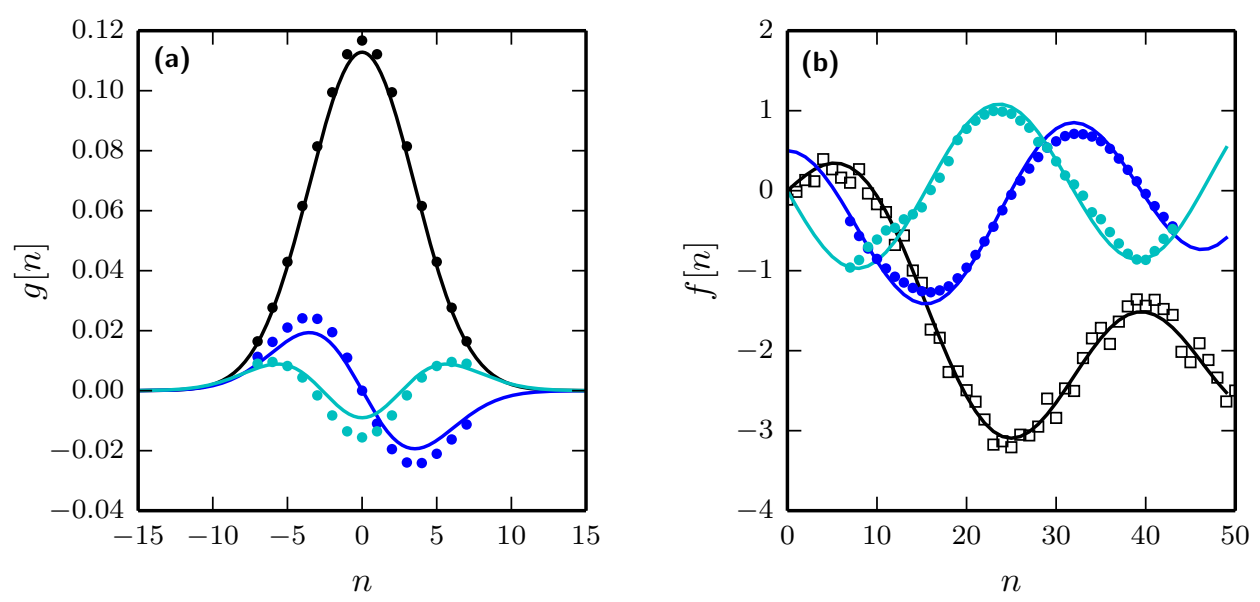

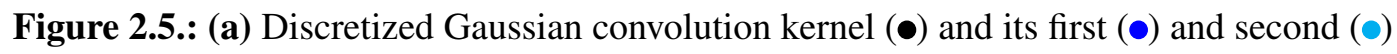
derivative. The corresponding continuous kernels are represented by solid lines. The filter length is $l=15$ and the width is $w=5$. (b) For an analytical test function $f(n)$ (black line), a corresponding noisy, discrete function $f[n]$ ( $\square$ ) was generated. The first $(\bullet)$ and second (๑) derivatives were then obtained with the convolution kernels shown in (a). The blue and cyan lines represent the true derivatives of $f(n)$.

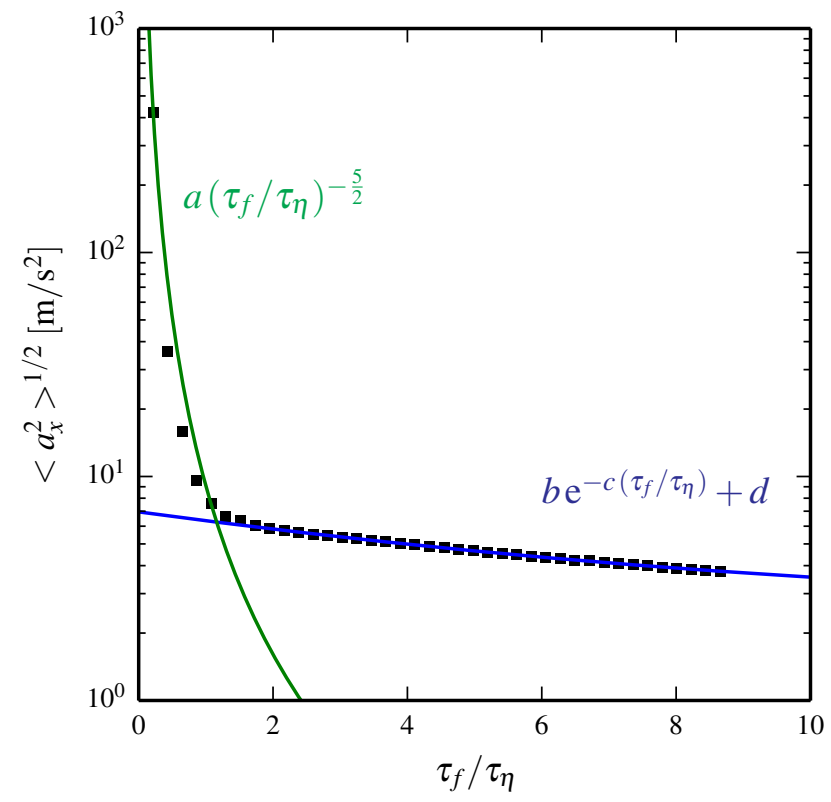

Figure 2.6.: Plot of the rms acceleration in the $x$ direction of a real dataset as a function of the temporal filter length. The ideal filter length is close to the crossover of the shown exponential fit for short $\tau_{f}$ and the power-law fit for large $\tau_{f}$. 
From the flow statistics, one then calculates the energy transfer rate $\varepsilon$, the energy injection scale $L$, the Reynolds number, and the Kolmogorov microscales $\eta$ and $\tau_{\eta}$. There are several options to compute the energy transfer rate. In section 1.2, it was shown that for an isotropic flow at the limit of infinite Reynolds number, the third-order longitudinal structure function is exactly given by $S_{3}(r)=-\frac{4}{5} \varepsilon r$ and the second-order longitudinal structure function is approximately $S_{2}(r)=C_{2}(\varepsilon r)^{\frac{2}{3}}$ with the Kolmogorov constant $C_{2} \approx 2.1$ (Sreenivasan 1995). Furthermore, for a homogeneous flow, $\langle\delta \mathbf{u} \cdot \delta \mathbf{a}\rangle=-2 \varepsilon$ as given in equation (1.16). With $\varepsilon$ and the rms velocity $u^{\prime}=\left\langle\frac{1}{3} \mathbf{u}^{2}\right\rangle^{\frac{1}{2}}$, the energy injection scale is simply given as $L=\frac{u^{\prime 3}}{\varepsilon}$, and for the Taylor scale Reynolds number ${ }^{4}$, one finds

$$
R_{\lambda}=\sqrt{15 \frac{u^{\prime 4}}{v \varepsilon}}
$$

The Kolmogorov microscales are then computed as defined by equation (1.12): $\eta=\left(v^{3} / \varepsilon\right)^{\frac{1}{4}}$ and $\tau_{\eta}=(\nu / \varepsilon)^{\frac{1}{2}}$.

\subsection{Experimental Setup}

The experimental setup consists mainly of three parts: A flow chamber, a laser for illumination, and three high-speed cameras with corresponding computational periphery, all depicted in figure 2.7. I will describe these hardware components briefly in section 2.3.1. The general measurement routine is described in section 2.3.2 and an overview of the calibration procedure is given in section 2.3.3.

\subsubsection{Hardware}

Three Phantom V12.1 high-speed cameras from Vision Research were used to obtain videos of the tracer particles. At a resolution of 640x640 pixels, videos can be taken at frame rates of up to 20000 frames per second (FPS). Since the cameras only contain an internal storage capacity of $8 \mathrm{~GB}$, it is necessary to download the videos after a short recording time. For this purpose, the cameras are connected to a network switch via a $1 \mathrm{Gbit} / \mathrm{s}$ Ethernet connection. From there, the data is transferred via a glass fiber cable to the in-house Linux cluster. With a download rate between 40 and $50 \mathrm{MB} / \mathrm{s}$, a video with a length of $1 \mathrm{~s}$ takes approximately 1 min to download.

For the illumination of the tracer particles, two different lasers with very similar specifications were used, of which only one will be described here: the Mirage 100DY from DDC Technologies ${ }^{5}$. It is a frequency doubled Nd:YAG laser with a wavelength of $532 \mathrm{~nm}$, used in a Q-switched mode with a pulse repetition rate of around $20 \mathrm{kHz}$. The laser is used at its

\footnotetext{
${ }^{4}$ For homogeneous, isotropic flows, the Taylor scale Reynolds number, $R_{\lambda}=\frac{u^{\prime} \lambda}{v}$, is often the preferred parameter to describe the flow. This is due to the fact that $R_{\lambda}$ depends on the Taylor scale, $\lambda=\sqrt{15 \mathrm{v} u^{\prime 2} / \varepsilon}$, which was historically easier to measure than the integral length scale, $L$ (see e.g. Pope 2000, chapter 6.3).

${ }^{5}$ The other laser was a self-built replicate of the Mirage 100DY, constructed by Haitao Xu, Fabio Di Lorenzo and myself. It generated a similar output power at the same wavelength and pulse rate.
} 


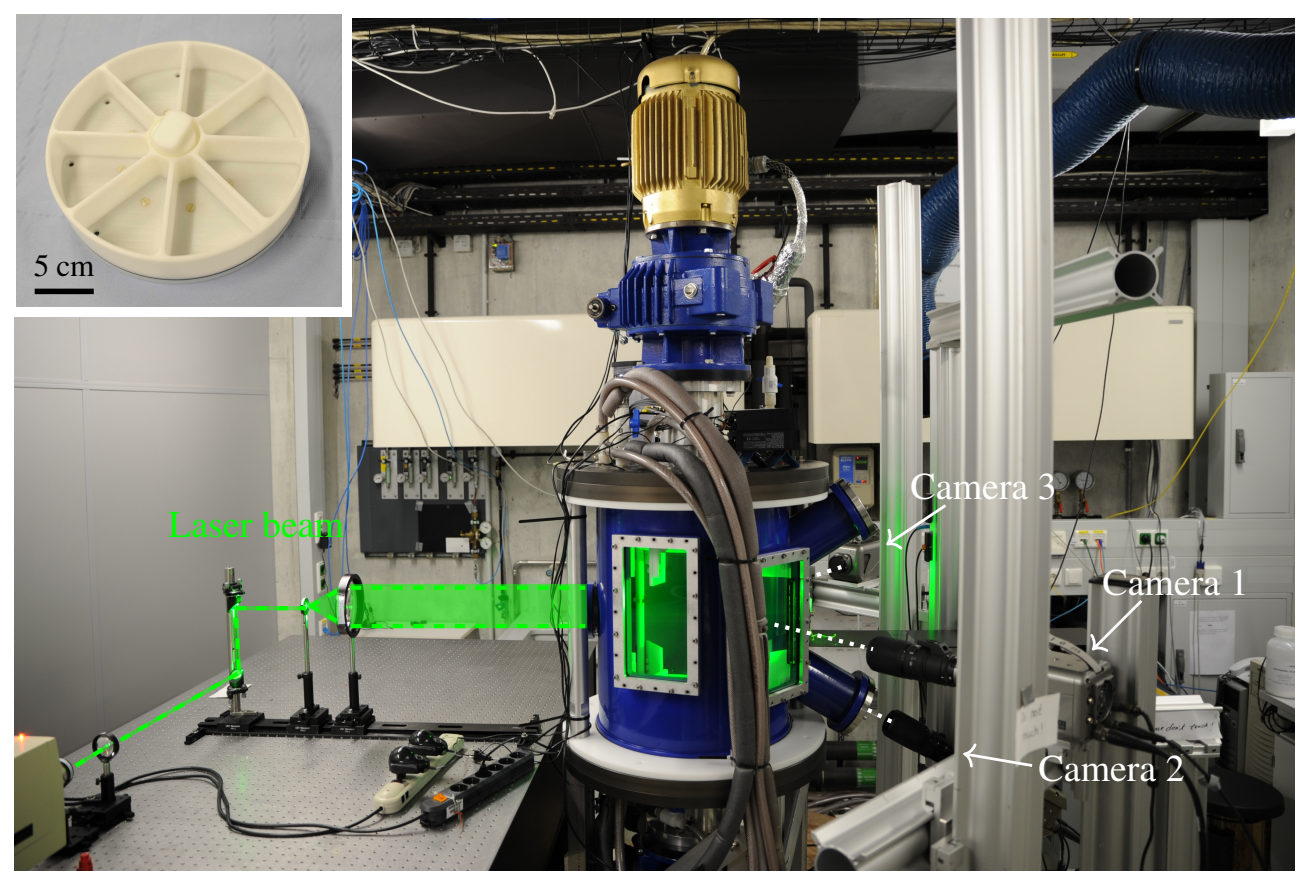

Figure 2.7.: A picture of the experimental setup. The lines of sight of the cameras are shown in white while the path of the laser beam is sketched in green. The inset shows one of the propellers.

highest power output of $P=38.0 \pm 1.0 \mathrm{~W}$. After exiting the laser head, the laser beam is collimated, expanded, and guided to an entrance window of the flow chamber (see figure 2.7).

The body of the flow chamber consists of a stainless steel cylinder with an inner diameter of $48 \mathrm{~cm}$ and a height of $58 \mathrm{~cm}$. Two circular glass windows with a diameter of $11 \mathrm{~cm}$ serve as entrance and exit points for the laser beam, thus forming an upper limit to the illuminable area. The laser beam passes horizontally through the center of the cylinder. Four windows, one each above, below, to the left, and to the right of the exit window, allow optical access of up to four cameras. For this thesis, cameras were positioned in front of all but the top window (see figure 2.7). A last access point is mounted at the side of the tank for maintenance purposes. All windows consist of two parallel sheets of glass, joined by simple glass spacers. While one sheet seals the tank, the other is positioned further inside, close to the inner tank surface. Water can enter the volume between the two sheets but the flow velocity in this region is close to zero. This way, the breaking of the rotational symmetry of the cylinder due to the window flanges is minimized.

The tank is closed by two cooling plates at the top and bottom. The plates have an internal spiral channel that is connected to an external chiller (ThermoFlex 2500 by Thermo Scientific) in order to keep the fluid temperature at a constant value of $20.5 \pm 0.5^{\circ} \mathrm{C}$. A thermometer in the top plate continuously measures the temperature inside the tank. Several tubes are connected to the top and bottom plates to fill or drain the tank and filter the water. Water may also be recirculated by a pump from the top to the bottom of the tank through an 


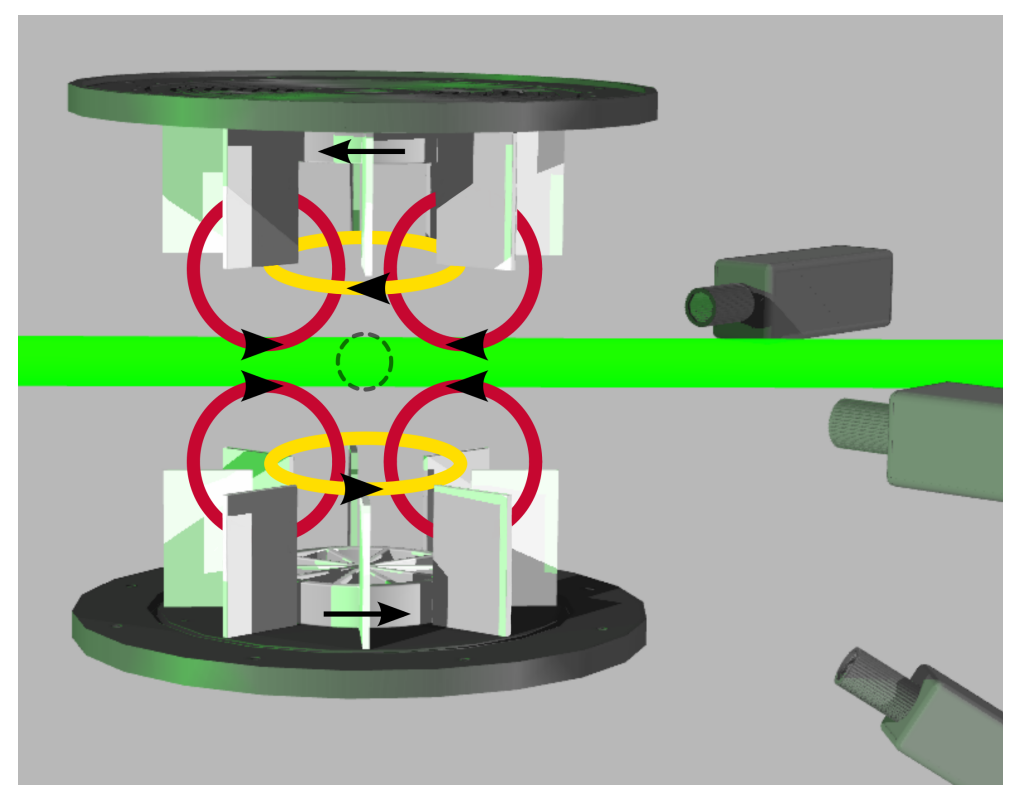

Figure 2.8.: Sketch of the flow chamber with the stainless steel cylinder removed. The flow field is shown by the colored arrows. The dashed circle in the center indicates the measurement area.

open reservoir. This way, bubbles can be removed and substances like additives or tracer particles can be introduced into the flow. Two counter-rotating propellers at the top and bottom of the cylinder produce the desired flow field. They have a disk-like shape with eight symmetrically distributed vanes (see insert in figure 2.7) and a total diameter of $25 \mathrm{~cm}$. Between the propellers and the cylinder wall, eight plastic inserts are installed to suppress a large-scale, rotational flow. The propellers are powered by two $7.5 \mathrm{~kW}$-motors and their rotation frequency is varied from $0.50 \mathrm{~Hz}$ to $1.10 \mathrm{~Hz}$ for the experiments shown in this work. The rotation frequency is recorded by proximity sensors at each propeller shaft. The error bounds on the frequency measurement are $\pm 0.01 \mathrm{~Hz}$. Figure 2.8 shows the characteristic flow field, a so-called Von Kármán Swirling Flow (for an overview see e.g Nore et al. 2003, and references therein). At the center region of the tank, where our observation area is positioned, the mean velocity field is negligible, while turbulent fluctuations are strong. Due to the finite size of the laser entrance window, the diameter of the observation area cannot exceed $9-10 \mathrm{~cm}$.

\subsubsection{Data Collection}

As described above, due to the limited internal storage of the cameras, only short videos can be recorded, and they must be download before proceeding with the next recording. It is thus beneficial to automate a repeating recording scheme and to use the download time for preprocessing. To this end, three nodes of the in-house Linux cluster are used to download and preprocess the videos, which are then stored on the cluster's internal hard 
drives. A server runs on each node to control communication with the cameras. A sketch of the network is shown in figure 2.9.

During the experiment, a master computer is used to control the cameras, the laser, and the communication with the nodes on the cluster. At the beginning of each video, the master computer opens the shutter of the laser via a relay and, after a short delay of approximately $2 \mathrm{~s}$, sends the start signal to the cameras. The delay allows the laser intensity to stabilize ${ }^{6}$. The cameras and laser are synchronized by a frequency generator. The frequency signal to the cameras is divided by a factor of either two or three, leading to multiple laser pulses per frame. The frequency signal to the laser, on the other hand, is delayed by $5 \mu$ s to ensure that no laser pulse is lost between frames. After a preset time of recording, usually $1 \mathrm{~s}$, the computer sends the stop signal to the cameras and closes the shutter. Then, the download of the videos to the cluster nodes is started. To do so, each node communicates with one of the cameras.

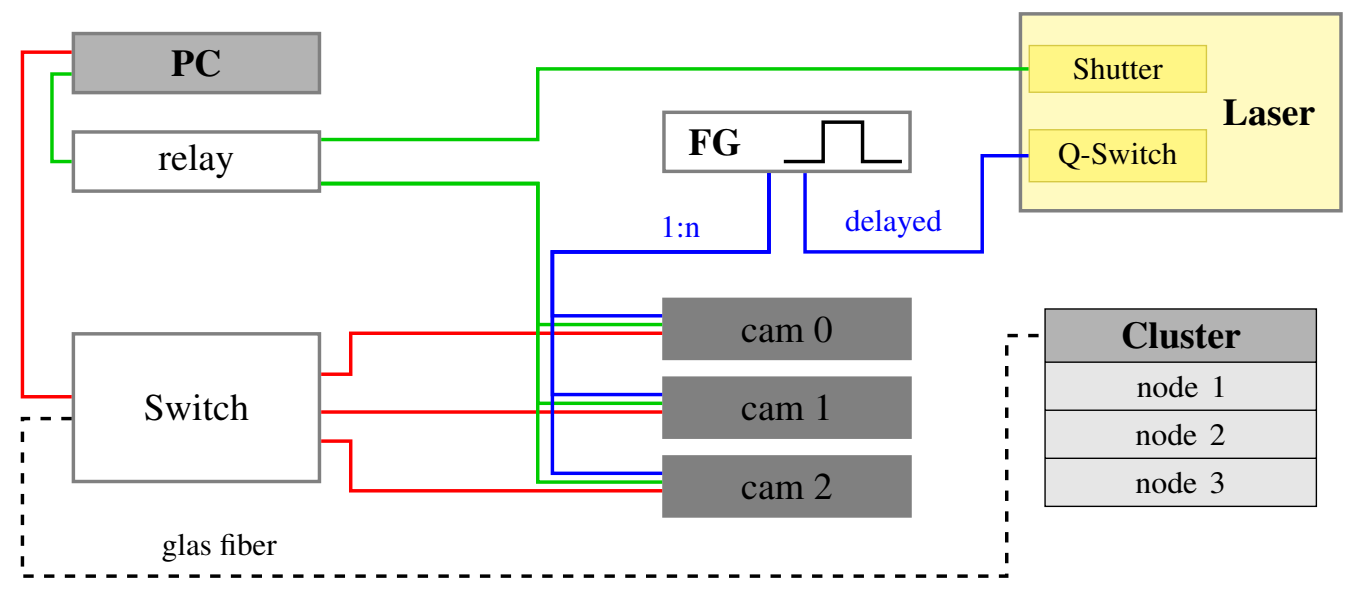

Figure 2.9.: Sketch of the network connecting the laser, the cameras, and the computers. Controlled by a computer (PC), a relay opens the shutter of the laser and, after some seconds, the PC starts the recording of a video. A frequency generator synchronizes the laser pulses with the start of each video frame. There are multiple laser pulses per frame with the first pulse shortly after the start of the frame. The recorded video can then be downloaded to the cluster where it is preprocessed and stored.

The cameras return the videos in a format providing a gray value for each pixel. For an 8 bit grayscale image, as used in this thesis, this results in a file size of approximately $400 \mathrm{kB}$ per frame for a resolution of 640x640 pixels. Since the images are sparse, a lot of space can be conserved by rewriting them in another format. For this purpose the videos are transformed during the download into the . gmv format (Göttingen Movie format, see appendix C.1). In the . gmv format, only bright pixels with an intensity above a chosen threshold ${ }^{7}$ are stored, together with the pixel coordinates. Each bright pixel thus needs 24 bit

\footnotetext{
${ }^{6}$ Since the pumping of the optical medium continues while the shutter is closed, the first pulse after opening the shutter has a very high intensity and the optical medium becomes nearly depleted. From there on, the laser intensity oscillates for some time until it stabilizes at a constant pulse intensity.

${ }^{7}$ The threshold is set according to the intensity histogram of a short test video recorded at the beginning of an
} 
of storage ( 16 bit for the coordinates, 8 bit for the intensity), compared to 8 bit of storage for each of the 409600 pixels of the uncompressed videos.

After completion of the download on all three nodes, the recording of a new video is initiated by the master computer.

\subsubsection{Calibration}

In section 2.1.2, the theoretical concept of the calibration procedure was described. In order to obtain the calibration parameters from equations (2.9), (2.10), and (2.13), the world and computer coordinates of several points in the measurement volume must be recorded. Experimentally, this is achieved by inserting a dot patterned mask into the center of the tank. The mask is positioned parallel to the symmetry axis of the tank and perpendicular to the laser path, thus defining the $y z$-plane of the world reference frame. Figure 2.10 shows images as recorded by the three cameras. Using a micrometer screw, the mask is then moved along the $x_{w}$ direction, taking images at fixed intervals. The coordinates of at least one hundred points, distributed in the whole intended observation volume, are recorded during one calibration measurement, leading to an over-determination of the calibration equations and thus very high precision.

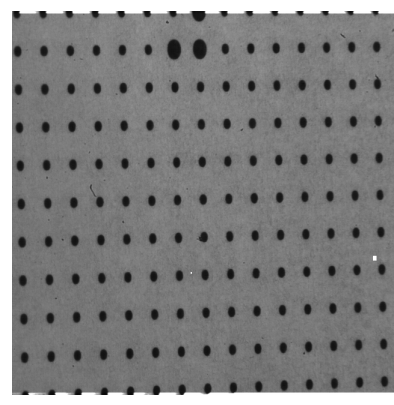

(a) Camera 1

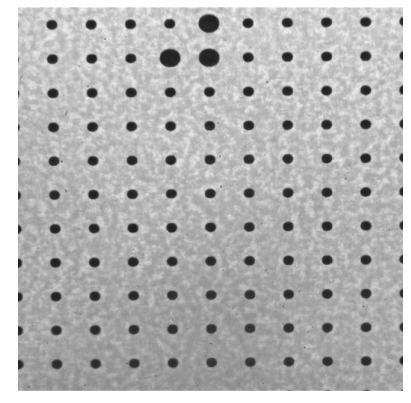

(b) Camera 2

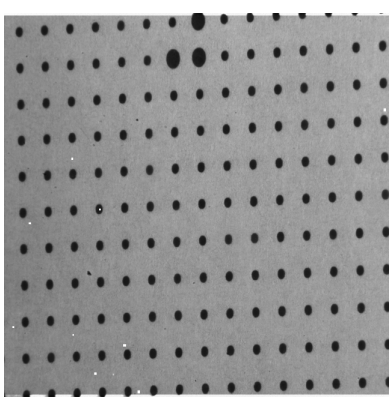

(c) Camera 3

Figure 2.10.: Calibration images from all three cameras with the mask being positioned at $x_{w}=0$. The three large, black dots are for orientation purposes inside the regular dot pattern. The dot pattern has a spacing of $7.62 \mathrm{~mm}$ in both the horizontal and vertical direction. Labeling of the cameras is according to figure 2.7.

\subsection{Datasets}

For this thesis, several datasets were recorded and, together with an already existing dataset, evaluated with respect to the dispersion of particle clusters, forwards and backwards in time. These datasets are briefly presented below, concerning both their experimental parameters and statistical properties. 
As a fluid, deionized water was used, either pure or with minute amounts of added polyacrylamide. The effect of the added polymer will be discussed in detail in chapter 5 . The kinematic viscosity of the pure deionized water is given by $v=0.99710^{-6} \frac{\mathrm{m}^{2}}{\mathrm{~s}}$ at $20^{\circ} \mathrm{C}^{8}$.

Datasets were recorded at three different propeller frequencies and three different concentrations of polyacrylamide. A set of two measurements was performed for each of the nine flow configurations: one with a small and one with a large measurement volume. The large measurement volume allows to track particle positions for a long time while the small measurement volume yields higher resolution data, usable for computing time derivatives and other high-precision quantities like the energy transfer rate. Table 2.1 shows the recording parameters for the small and large measurement volume as well as the already existing dataset.

\begin{tabular}{lccccl}
\hline & $\varnothing V[\mathrm{~cm}]$ & resolution & $\delta_{x}[\mu \mathrm{m}]$ & $\delta t[\mathrm{~ms}]$ & $d[\mu \mathrm{m}]$ \\
\hline small Volume & 2.7 & $640 \times 640$ & 10 & 0.10 & 45 \\
large Volume & 8.0 & $640 \times 640$ & 50 & 0.14 & 80 \\
\hline existing data $^{9}$ & 5.0 & $256 \times 256$ & 40 & 0.04 & 25 \\
\hline
\end{tabular}

Table 2.1.: Experimental recording parameters for the used datasets. The diameter of the measurement volume, $\varnothing V$, is estimated by fitting the largest possible sphere into the area visible by all three cameras. The accuracy of the $3 \mathrm{D}$ center finding is given by $\delta_{x}$, which is obtained from the calibration measurement. For more than $90 \%$ of the $3 \mathrm{D}$ calibration points, the maximum distance between the projections from the different cameras is smaller than $\delta_{x}$. The time lapse between two successive frames, $\delta t$, is a measure for the temporal resolution. The mean diameter of the tracer particles, $d$, is given in the last column.

As discussed in section 2.2, there are three ways to compute the energy transfer rate. One can use the structure functions, $S_{2}(r)=C_{2}(\varepsilon r)^{\frac{2}{3}}$ and $S_{3}(r)=-\frac{4}{5} \varepsilon r$, or the relation $\langle\delta \mathbf{u} \cdot \delta \mathbf{a}\rangle=-2 \varepsilon$. For the functional expressions of the structure functions, homogeneity, isotropy, and a very large Reynolds number were assumed. For $\langle\delta \mathbf{u} \cdot \delta \mathbf{a}\rangle$, on the other hand, only homogeneity is required and it was shown experimentally that moderate Reynolds numbers are sufficient (see Ouellette et al. 2006b, Figure 7).

The experimental flow studied in this thesis is only approximately isotropic and homogeneous, with the magnitude of the mean flow being up to $20 \%$ of the magnitude of the turbulent velocity fluctuations in the measurement volume (see appendix B.1). Furthermore, only moderate Reynolds numbers are reached. This suggests that $\langle\delta \mathbf{u} \cdot \delta \mathbf{a}\rangle$ should yield the best result for the energy transfer rate, $\varepsilon$. Figure 2.11 shows all three ways to obtain $\varepsilon$ for a dataset with a propeller frequency of $0.9 \mathrm{~Hz}$ and without polymers. The measured quantities

\footnotetext{
${ }^{8}$ The kinematic viscosity of the polymer solutions is close to the one of the water case. It's exact value is unimportant, however, since it is not needed for any further calculation.

${ }^{9}$ This dataset was recorded by Nicholas Ouellette at a previous version of the French Washing Machine described above. For further information see (Ouellette 2006).
} 


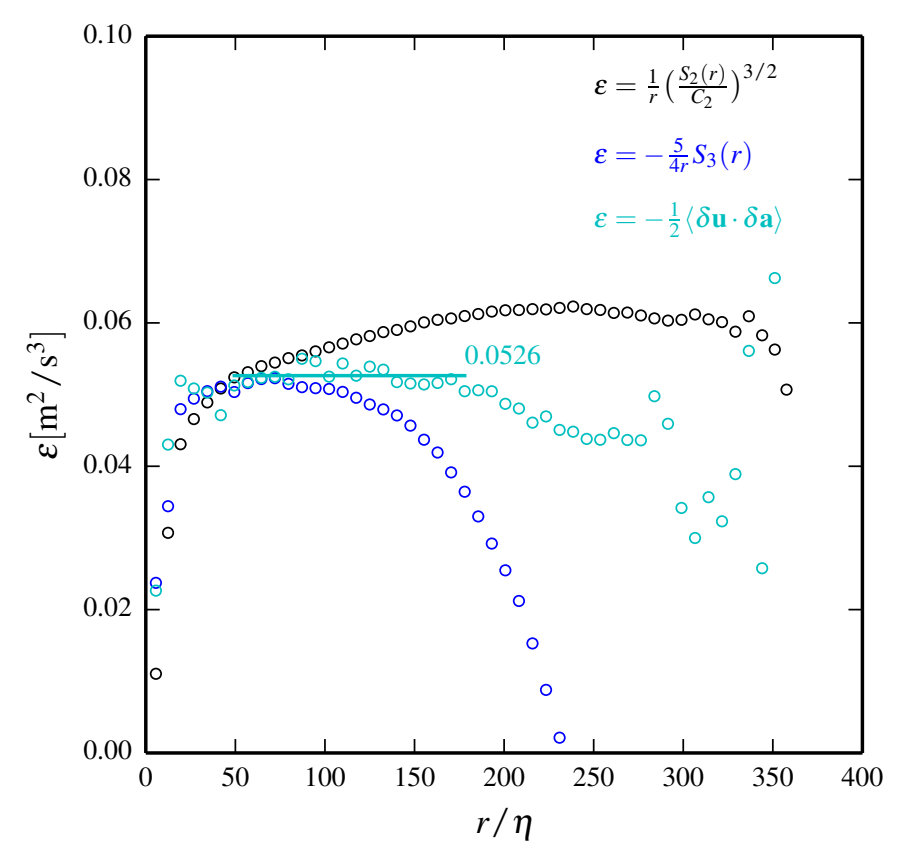

Figure 2.11.: Three different ways to obtain the energy transfer rate. The shown dataset corresponds to a flow with a propeller frequency of $0.9 \mathrm{~Hz}$ and without polymers. The horizontal line depicts the mean value of $-\frac{1}{2}\langle\delta \mathbf{u} \cdot \delta \mathbf{a}\rangle$ in the range $50 \eta \leq r \leq \frac{1}{5} L$.

are compensated in such a way that they should show a plateau with height $\varepsilon$ in the inertial range. It can be clearly seen that, while the structure functions do not display a plateau, $\langle\delta \mathbf{u} \cdot \delta \mathbf{a}\rangle$ shows a constant, if somewhat noisy, value for $\varepsilon$ over a range of scales. The energy transfer rates for all flow configurations studied in this thesis were thus obtained by fitting a horizontal line to the plateau of the compensated expression for $\langle\delta \mathbf{u} \cdot \delta \mathbf{a}\rangle$ in the range $50 \eta \leq r \leq \frac{1}{5} L$, using the data from the small measurement volume ${ }^{10}$. The uncertainty on the energy transfer rate is estimated to be $10 \%$ (see section 2.5). Please note, however, that the mean flow becomes more dominant with an increasing distance from the tank center, so that the mean energy transfer rate measured from the large measurement volume experiment can differ up to $20 \%$ from the small measurement volume experiment ${ }^{11}$. On the other hand, due to the larger volume, finite volume effects are smaller and the plateau of $\langle\delta \mathbf{u} \cdot \delta \mathbf{a}\rangle$ extends beyond $20 \mathrm{~mm}(\sim L / 3)$, indicating the true extend of the inertial range. All $n$-particle statistics discussed in this theses have initial particle separations in the such defined inertial range. Since the statistics of $n$-particle clusters increase with an increasing separation of

\footnotetext{
${ }^{10}$ For the already existing dataset, the fit range was $50 \eta \leq r \leq \frac{1}{10} L$. For the cases with added polymers, the lower bound of the fit range was increased to account for a shift of the plateau to higher values. Furthermore, since $\eta$ may not be a valid quantity for the polymers, the bounds were chosen in an uncompensated way with the lower bound in the range $9-13 \mathrm{~mm}$ and the upper bound at approximately $16 \mathrm{~mm}$.

11 The measured rms velocity has an estimated uncertainty of only $3 \%$ while the rms velocities obtained from the small and large measurement volume can differ up to $10 \%$.
} 
the particles (as long as the separation is smaller than approximately half the diameter of the measurement volume), I chose to study clusters with initial particle separations of 14 , 16 and $18 \mathrm{~mm}$ for the large measurement volume, and with initial separations of $8,10,12$ and $14 \mathrm{~mm}$ for the small volume ${ }^{12}$. This way, I ensure that my results are best possibly statistically resolved with the only drawback that the overlap in scales between the large and small measurement volume results is small.

With $v$ and $\varepsilon$ known, further quantities can be computed as explained in section 2.2. Table 2.2 finally summarizes the most important properties of the analyzed flows.

\begin{tabular}{cccccccc}
\hline$f[\mathrm{~Hz}]$ & $c[\mathrm{ppm}]^{13}$ & $u^{\prime}[\mathrm{m} / \mathrm{s}]$ & $\varepsilon\left[\mathrm{m}^{2} / \mathrm{s}^{3}\right]$ & $R_{\lambda}$ & $L[\mathrm{~mm}]$ & $\eta[\mu \mathrm{m}]$ & $\tau_{\eta}[\mathrm{ms}]$ \\
\hline $0.50 \pm 0.01$ & 0 & 0.079 & $8.410^{-3}$ & 270 & 59 & 104 & 10.9 \\
$0.90 \pm 0.01$ & 0 & 0.144 & $5.310^{-2}$ & 350 & 54 & 66 & 4.4 \\
$1.10 \pm 0.01$ & 0 & 0.174 & $9.010^{-2}$ & 390 & 57 & 58 & 3.3 \\
\hline $0.50 \pm 0.01$ & $5.0_{-0.2}^{+0.5}$ & 0.074 & $\left(5.510^{-3}\right)$ & & & \\
$0.90 \pm 0.01$ & $5.0_{-0.2}^{+0.5}$ & 0.129 & $3.210^{-2}$ & not defined & \\
$1.1 \pm 0.01$ & $5.0_{-0.2}^{+0.7}$ & 0.164 & $6.710^{-2}$ & & & \\
\hline $0.50 \pm 0.01$ & $10.0 \pm 0.3$ & 0.062 & $\left(3.010^{-3}\right)$ & & \\
$0.90 \pm 0.01$ & $10.0 \pm 0.3$ & 0.126 & $2.810^{-2}$ & & & \\
$1.10 \pm 0.01$ & $10.0_{-0.3}^{+0.4}$ & 0.151 & $5.710^{-2}$ & & & \\
\hline $3.50 \pm 0.01$ & 0 & 0.430 & 0.87 & 690 & 91 & 33 & 1.1 \\
\hline
\end{tabular}

Table 2.2.: Physical properties of the used datasets: Propeller frequency $f$, polymer concentration $c$, rms velocity $u^{\prime}$, energy transfer rate $\varepsilon$, Taylor scale Reynolds number $R_{\lambda}$, and Kolmogorov microscales $\eta$ and $\tau_{\eta}$. The error bounds for $f$ and $c$ are given in the table. The uncertainties for $u^{\prime}$ and $\varepsilon$ are given by $\frac{\Delta u^{\prime}}{u^{\prime}}=3 \%$ and $\frac{\Delta \varepsilon}{\varepsilon}=10 \%$, respectively, leading to $\frac{\Delta R_{\lambda}}{R_{\lambda}}=8 \%, \frac{\Delta L}{L}=13 \%, \frac{\Delta \eta}{\eta}=3 \%$ and $\frac{\triangle \tau_{\eta}}{\tau_{\eta}}=5 \%$. For the $0.5 \mathrm{~Hz}$ datasets with added polymers, $\langle\boldsymbol{\delta} \mathbf{u} \cdot \boldsymbol{\delta} \mathbf{a}\rangle$ doesn't show a convincing plateau anymore. The $\varepsilon$-values for these datasets have thus to be treated with caution (see appendix A.1). The Reynolds number and Kolmogorov microscales are computed for all three propeller frequencies for the water case and then adopted without change for the flows with added polymers. The physical parameters for the already existing dataset, given in the last row, have been computed in the same way.

\footnotetext{
${ }^{12}$ The already existing dataset is only used in the case of four-particle clusters. There, initial separations of $12 \mathrm{~mm}, 14 \mathrm{~mm}$, and $16 \mathrm{~mm}$ are studied.

${ }^{13}$ The varying error bounds for the concentration are due to a gradual improvement of the funneling procedure. The bounds shown here are for the large volume measurements. The small volume measurements, conducted afterwards, have error bounds of $\pm 0.3 \mathrm{ppm}$ for both concentrations. For more details see chapter 5 .
} 


\subsection{Measurement Uncertainties}

A rigorous calculation of the measurement uncertainties of the presented results is not feasible with standard methods. This is due to a complicated confounding of different uncertainties, as well as a lack of statistical independence between individual measurements, from now on called realizations. There are three main sources for uncertainties:

1. Every 3D position comes with a measurement error of the order of $\delta x$ (see table 2.1).

2. Smoothing the trajectory with a Gaussian kernel, as described in section 2.2, picks up the position error and introduces a slight systematic error towards smaller amplitudes of the trajectory.

3. Each studied quantity is computed from the positions of one or more particles and then averaged over many realizations. This introduces a statistical uncertainty. If each realization was taken from another recorded video, they would all be statistically independent and the mean value would be Gaussian distributed according to the law of large numbers. For a turbulent flow, however, the number of realizations averaged over must be very large in order to achieve a statistical convergence. Therefore, all realizations from each video must be used in order to collect sufficient data in a realistic timespan. This renders the realizations statistically dependent and the mean value is not necessarily Gaussian distributed.

As an example for the effect of these sources on the uncertainty of a measured quantity, I present a thorough analysis of the energy transfer rate obtained from $\langle\delta \mathbf{u} \cdot \delta \mathbf{a}\rangle$ as was shown in figure 2.11. The three sources of uncertainties stated above are discussed in the given order, and an optimistic and a very conservative estimate will be discussed. Quantities with a possible uncertainty are marked with a tilde while true values are written without.

1. The measured position of a particle at frame $k$ can be written as $\tilde{\mathbf{x}}[k]=\mathbf{x}[k]+\xi_{x}[k]$ with $\xi_{x}[k]$ the measurement error at frame $k$. The absolute value of $\xi_{x}[k]$ should be of the order of the accuracy of the 3D center finding. For the dataset shown in figure 2.11, this is $\delta_{x}=10 \mu \mathrm{m}$ (see table 2.1).

2. For $\langle\delta \mathbf{u} \cdot \delta \mathbf{a}\rangle$, both the velocity as well as the acceleration need to be computed from the particle positions. According to section 2.2, this is accomplished by using a convolution with the respective derivative of a Gaussian kernel. For the velocity at frame $k$, one obtains

$$
\begin{aligned}
\tilde{\mathbf{u}}[k] & =\frac{1}{\delta t} \sum_{m=-M}^{M} \tilde{\mathbf{x}}[k-m] g^{\prime}[m] \\
& =\frac{1}{\delta t} \sum_{m=-M}^{M} \mathbf{x}[k-m] g^{\prime}[m]+\frac{1}{\delta t} \sum_{m=-M}^{M} \xi_{x}[k-m] g^{\prime}[m],
\end{aligned}
$$

where the notation for the position error from above was used at the second equality. The first term in equation (2.22) leads to the true value of the velocity plus an error 
due to the filtering process which I will write as $\xi_{u, f}[k]$. The second term picks up the position errors and transforms them into a cumulated error, $\xi_{u, x}[k]$. The error due to filtering, $\xi_{u, f}$, is unknown. However, using numerical data, Ouellette (2006) showed that its effect on the velocity variance is less than $1 \%$ for a large range of filter lengths and can thus be neglected. The cumulated position error, $\xi_{u, x}$, on the other hand, can be estimated analytically under the assumption that the position error is white in time and stationary, meaning that $\left\langle\xi_{x}[k+n] \cdot \xi_{x}[k+m]\right\rangle=(\delta x)^{2} \delta_{n m}$ with $\delta_{n m}$ being the Kronecker delta. The variance of the cumulated position error is then given by

$$
\left\langle\xi_{u, x}^{2}\right\rangle^{\frac{1}{2}}=\frac{\delta x}{\delta t}\left(\sum_{m=-M}^{M}-m^{2} \mathrm{e}^{-\frac{m^{2}}{w^{2}}}\right)^{-1}\left(\sum_{m=-M}^{M} m^{2} \mathrm{e}^{-2 \frac{n^{2}}{w^{2}}}\right)^{\frac{1}{2}}=0.0024 \frac{\mathrm{m}}{\mathrm{s}},
$$

where the parameters for the dataset in figure 2.11 were used at the second equality: $\delta x=10 \mu \mathrm{m}, \delta t=0.10 \mathrm{~ms}, M=15$, and $w=10$. The obtained cumulated position error corresponds to approximately $2 \%$ of $u^{\prime}$. For the acceleration one finds similarly

$$
\begin{aligned}
\left\langle\xi_{a, x}^{2}\right\rangle^{\frac{1}{2}}=\frac{\delta x}{(\delta t)^{2}}\left(\sum_{m=-M}^{M} \frac{m^{2}}{2} g_{0}^{\prime \prime}[m]-\sum_{m=-M}^{M} \frac{m^{2}}{2} \sum_{m=-M}^{M} g_{0}^{\prime \prime}[m]\right)^{-1} & \left(\sum_{m=-M}^{M}\left[g_{0}^{\prime \prime}[m]-\frac{1}{2 M+1} \sum_{m=-M}^{M} g_{0}^{\prime \prime}[m]\right]^{2}\right)^{\frac{1}{2}}=5.9 \frac{\mathrm{m}}{\mathrm{s}^{2}},
\end{aligned}
$$

which is of the order of $20 \%$ of the rms acceleration. The filtering error for the acceleration, $\xi_{a, f}$, is unknown but assumed to be negligible as for the velocity case. Therefore, one obtains $\tilde{\mathbf{u}}(\tilde{\mathbf{x}}, t) \approx \mathbf{u}(\tilde{\mathbf{x}}, t)+\xi_{u, x}(\tilde{\mathbf{x}}, t)$ and $\tilde{\mathbf{a}}(\tilde{\mathbf{x}}, t) \approx \mathbf{a}(\tilde{\mathbf{x}}, t)+\xi_{a, x}(\tilde{\mathbf{x}}, t)$.

3. Averaging over many pairs with a certain separation $r_{i}$ yields

$$
\begin{aligned}
\tilde{\varepsilon}\left(r_{i}\right)=-\frac{1}{2}\langle[\tilde{\mathbf{u}}(\tilde{\mathbf{x}}+\tilde{\mathbf{r}}, t)-\tilde{\mathbf{u}}(\tilde{\mathbf{x}}, t)] \cdot[\tilde{\mathbf{a}}(\tilde{\mathbf{x}}+\tilde{\mathbf{r}}, t)-\tilde{\mathbf{a}}(\tilde{\mathbf{x}}, t)]\rangle_{|\tilde{\mathbf{r}}|=r_{i}} \\
=-\frac{1}{2}\langle[\mathbf{u}(\tilde{\mathbf{x}}+\tilde{\mathbf{r}}, t)-\mathbf{u}(\tilde{\mathbf{x}}, t)] \cdot[\mathbf{a}(\tilde{\mathbf{x}}+\tilde{\mathbf{r}}, t)-\mathbf{a}(\tilde{\mathbf{x}}, t)]\rangle_{|\tilde{\mathbf{r}}|=r_{i}} \\
+\left\langle\left[\xi_{u, x}(\tilde{\mathbf{x}}+\tilde{\mathbf{r}}, t)-\xi_{u, x}(\tilde{\mathbf{x}}, t)\right] \cdot[\mathbf{a}(\tilde{\mathbf{x}}+\tilde{\mathbf{r}}, t)-\mathbf{a}(\tilde{\mathbf{x}}, t)]\right\rangle_{|\tilde{\mathbf{r}}|=r_{i}} \\
+\left\langle[\mathbf{u}(\tilde{\mathbf{x}}+\tilde{\mathbf{r}}, t)-\mathbf{u}(\tilde{\mathbf{x}}, t)] \cdot\left[\xi_{a, x}(\tilde{\mathbf{x}}+\tilde{\mathbf{r}}, t)-\xi_{a, x}(\tilde{\mathbf{x}}, t)\right]\right\rangle_{|\tilde{\mathbf{r}}|=r_{i}} \\
+\left\langle\left[\xi_{u, x}(\tilde{\mathbf{x}}+\tilde{\mathbf{r}}, t)-\xi_{u, x}(\tilde{\mathbf{x}}, t)\right] \cdot\left[\xi_{a, x}(\tilde{\mathbf{x}}+\tilde{\mathbf{r}}, t)-\xi_{a, x}(\tilde{\mathbf{x}}, t)\right]\right\rangle_{|\tilde{\mathbf{r}}|=r_{i}}
\end{aligned}
$$

where the average $\langle\cdot\rangle_{|\tilde{\mathbf{r}}|=r_{i}}$ is taken over pairs with a separation between $r_{i}$ and $r_{i}+\delta r_{i}$. For the data shown in figure 2.11, the bin width, $\delta r_{i}$, was chosen to be $0.5 \mathrm{~mm}$. It can be assumed that the correlation between the cumulated position error for the velocity, $\xi_{u, x}$, and the acceleration is negligible, and the same for $\xi_{a, x}$ and the velocity. For the last term, containing the mixed correlation between the cumulated position errors, an analytical result can be found, again assuming that the position errors are white in 
time. One finds that

$$
\left\langle\xi_{u, x} \cdot \xi_{a, x}\right\rangle=\frac{(\delta x)^{2}}{(\delta t)^{3}} \sum_{m=-M}^{M} g^{\prime}[m] g^{\prime \prime}[m]=0,
$$

where the last equality holds because $\left(g^{\prime}[m] g^{\prime \prime}[m]\right)$ is asymmetric in $m$ and the sum is over a symmetric interval. Even if the position errors are not perfectly white in time but rather have a short but finite correlation time, the above mixed term remains small. With these assumptions, equation (2.25) can be simplified to

$$
\tilde{\varepsilon}\left(r_{i}\right)=-\frac{1}{2}\langle[\mathbf{u}(\mathbf{x}+\mathbf{r}, t)-\mathbf{u}(\mathbf{x}, t)] \cdot[\mathbf{a}(\mathbf{x}+\mathbf{r}, t)-\mathbf{a}(\mathbf{x}, t)]\rangle_{|\mathbf{r}|=r_{i}} .
$$

Here, I made also use of the fact that the deviations of the measured position vector, $\tilde{\mathbf{x}}$, and separation vector, $\tilde{\mathbf{r}}$, from the true values has a vanishing effect on the result due to homogeneity and the large bin size, $\delta r_{i}$. Note that the easy solution found in equation (2.27) is the exception and not the rule. For any squared quantity, like e.g. the squared relative velocity, $\left\langle[\tilde{\mathbf{u}}(\tilde{\mathbf{x}}+\tilde{\mathbf{r}}, t)-\tilde{\mathbf{u}}(\tilde{\mathbf{x}}, t)]^{2}\right\rangle_{|\tilde{\mathbf{r}}|=r_{i}}$, the term with the squared uncertainties does not vanish but leads to a systematic error towards larger values.

For an ensemble of $N$ statistically independent realizations, the uncertainty of the average in equation (2.27) is given by

$$
\sigma_{\varepsilon}\left(r_{i}\right)=\frac{1}{\sqrt{N}}\left\langle\left[-\frac{1}{2}([\mathbf{u}(\mathbf{x}+\mathbf{r}, t)-\mathbf{u}(\mathbf{x}, t)] \cdot[\mathbf{a}(\mathbf{x}+\mathbf{r}, t)-\mathbf{a}(\mathbf{x}, t)])_{|\mathbf{r}|=r_{i}, j}-\tilde{\varepsilon}\left(r_{i}\right)\right]^{2}\right\rangle^{\frac{1}{2}} .
$$

A very optimistic estimate would be to say that all averaged over realizations of particle pairs are statistically independent, leading to $N=\mathcal{O}\left(10^{6}\right)$ for the data set studied here. This is not true, however, since realizations from the same video can be strongly correlated, depending on how far they are apart in space and time. A much more conservative approach would thus be to only allow one (randomly chosen) realization per video, leading to $N=400$. It is clear that statistical convergence at this small number of realizations is very poor. Figure 2.12 shows the results for $\tilde{\varepsilon}\left(r_{i}\right)$ for both cases. Open black circles and red error bars correspond to $N=\mathcal{O}\left(10^{6}\right)$ while filled black circles and blue error bars correspond to $N=400$. The error bars represent $2 \sigma_{\varepsilon\left(r_{i}\right)}$, so that they should include the true value in $95.4 \%$ of the cases. Figure 2.12 reveals that both methods agree with each other within the given errors. While the $N=400$ case clearly shows a severe lack of statistical convergence, however, the $N=\mathcal{O}\left(10^{6}\right)$ case seems well converged but its error bars are utterly underestimated. They do not even cover the fluctuations of the curve. As a result, the average value of the case with $N=\mathcal{O}\left(10^{6}\right)$ should be trusted, but its true uncertainties, $\sigma_{\varepsilon}\left(r_{i}\right)$, must be somewhere between these two extremes. Since the true uncertainties remain unknown, any display of error bars is misleading because it does not represent the actual uncertainty of the shown value. I will thus refrain from showing error bars in this thesis. 
One is left with the problem to find the value and uncertainty of the mean energy transfer rate, $\tilde{\varepsilon}$. Using the data points from the $N=\mathcal{O}\left(10^{6}\right)$ case, the value of $\tilde{\varepsilon}$ is given by $\tilde{\varepsilon}=\frac{1}{K} \sum_{i=1}^{K} \tilde{\varepsilon}\left(r_{i}\right)=0.05264 \frac{\mathrm{m}^{2}}{\mathrm{~s}^{3}}$, with $K$ the number of bins averaged over. Taking error propagation into account, the uncertainty is given by

$$
\sigma_{\varepsilon}=\frac{1}{K}\left(\sum_{i=1}^{K} \sigma_{\varepsilon}\left(r_{i}\right)^{2}\right)^{\frac{1}{2}}
$$

When using the case with $N=\mathcal{O}\left(10^{6}\right)$ realizations, one obtains $\sigma_{\varepsilon}=0.00006 \frac{\mathrm{m}^{2}}{\mathrm{~s}^{3}}$ which corresponds to a relative uncertainty of $0.1 \%$. On the other hand, for $N=400$, one finds $\sigma_{\varepsilon}=0.02 \frac{\mathrm{m}^{2}}{\mathrm{~s}^{3}}$, leading to a relative uncertainty of $37 \%$. These results reinforce the finding from above that neither the conservative nor the optimistic estimate for the uncertainties are very realistic. The total width of the curve fluctuations in the inertial range might provide a more accurate estimate for the uncertainty of $\varepsilon$. The standard deviation from the mean is given by $\sigma_{\text {scatter }}=\left(\frac{1}{K-1} \sum_{i=1}^{K}\left(\tilde{\varepsilon}\left(r_{i}\right)-\tilde{\varepsilon}\right)^{2}\right)^{\frac{1}{2}}$. I will thus use $4 \sigma_{\text {scatter }}=0.005 \frac{\mathrm{m}^{2}}{\mathrm{~s}^{3}}$, covering more than $99.99 \%$ of the fluctuations, as an estimate for the uncertainty of $\tilde{\varepsilon}$. This corresponds to a relative uncertainty of $10 \%$, as was stated above.

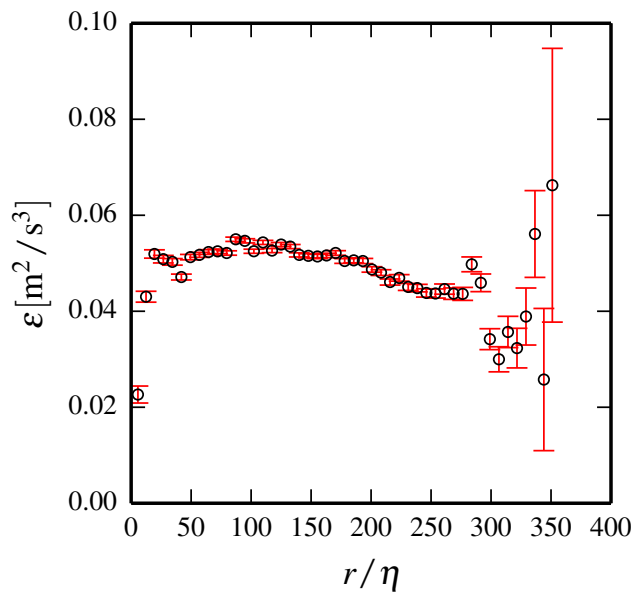

(a) Optimistic Estimate

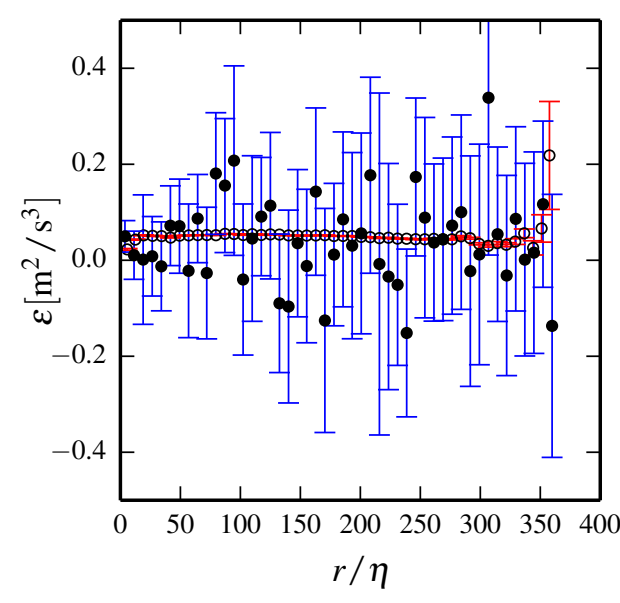

(b) Conservative Estimate

Figure 2.12.: Two ways to estimate the uncertainty for the energy transfer rate computed from $\langle\delta \mathbf{u} \cdot \delta \mathbf{a}\rangle$. The shown dataset corresponds to a flow with a propeller frequency of $0.9 \mathrm{~Hz}$ and without polymers. a) Data points (०) and red error bars are computed from $N=\mathcal{O}\left(10^{6}\right)$ realizations, assuming that they are all independent. The black horizontal line represents the mean value of the compensated squared relative velocities in the range $50 \eta \leq r \leq \frac{1}{5} L$. b) Data points $(\bullet)$ and blue error bars are computed from $N=400$ realizations, taking only one realization from each video to ensure statistical independence. For comparison, the results from a) are shown as well. 


\section{CHAPTER 3}

\section{Two-Particle Dispersion}

In this chapter, I analyze the relative dispersion of a pair of tracer particles with respect to the time asymmetry induced by the irreversibility of turbulent flow. In section 3.1, I use a short-time expansion to obtain a mathematical connection between the mean squared relative separation of a particle pair and the derivative of the squared relative velocity. I briefly explain how this connection can be understood in terms of the irreversibility of the energy cascade and how it determines the time asymmetry observed in pair dispersion. In section 3.2, I describe how the recorded particle track data is analyzed in order to obtain the required time-dependent pair statistics and in section 3.3, I present the experimental results and compare them with data from direct numerical simulation (DNS) ${ }^{1}$. Furthermore, I discuss how the finite size of the observation volume leads to a bias of the recorded data and explain which quantities are least affected.

\subsection{Short-Time Expansion}

Due to the elusive nature of Richardson scaling (Bourgoin et al. 2006, Salazar \& Collins 2009, Bragg et al. 2014), it seems prudent to focus on the short-time behavior of pair dispersion instead. For this purpose, I examine the mean squared relative separation of pairs of tracer particles, $\left\langle\delta \mathbf{R}^{2}(t)\right\rangle=\left\langle[\mathbf{R}(t)-\mathbf{R}(0)]^{2}\right\rangle$, where the average is taken over all pairs with the same initial separation $|\mathbf{R}(0)|=R_{0}$. In this thesis, $R_{0}$ is always chosen to be in the inertial range.

Recalling that the separation vector for a pair of particles is defined as $\mathbf{R}(t)=\mathbf{X}\left(t \mid \mathbf{y}_{2}, 0\right)-$ $\mathbf{X}\left(t \mid \mathbf{y}_{1}, 0\right)$, the short-time evolution of the mean squared relative separation can be expressed

\footnotetext{
${ }^{1}$ The numerical data was provided by Alain Pumir from the École Normale Supérieure, Lyon, and was also used in our joint paper (Jucha et al. 2014). For more details see section 3.3.
} 
by the Taylor series around $t=0$ as

$$
\left\langle\delta \mathbf{R}(t)^{2}\right\rangle=\left\langle\mathbf{V}(0)^{2}\right\rangle t^{2}+\langle\mathbf{V}(0) \cdot \mathbf{A}(0)\rangle t^{3}+\mathcal{O}\left(t^{4}\right) .
$$

Here, $\mathbf{V}(0)=\mathbf{u}\left(\mathbf{y}_{2}, 0\right)-\mathbf{u}\left(\mathbf{y}_{1}, 0\right)$ is the initial relative velocity between the particles and $\mathbf{A}(0)=\mathbf{a}\left(\mathbf{y}_{2}, 0\right)-\mathbf{a}\left(\mathbf{y}_{1}, 0\right)$ is the initial relative acceleration. In the case of an isotropic turbulent flow, the first term on the right hand side corresponds to Batchelor scaling and can be replaced by $\frac{11}{3} C_{2}\left(\varepsilon R_{0}\right)^{\frac{2}{3}} t^{2}$, in accordance with equation (1.23). Furthermore, the second term can be recognized to be the Lagrangian expression for $\langle\delta \mathbf{u} \cdot \delta \mathbf{a}\rangle$, with

$$
\langle\mathbf{V}(0) \cdot \mathbf{A}(0)\rangle=\frac{1}{2}\left\langle\left.\frac{\mathrm{d}}{\mathrm{d} t} \mathbf{V}(t)^{2}\right|_{t=0}\right\rangle=-2 \varepsilon
$$

as shown in equation (1.27). With this, equation (3.1) can be conveniently written as

$$
\left\langle\delta \mathbf{R}(t)^{2}\right\rangle=\frac{11}{3} C_{2}\left(\varepsilon R_{0}\right)^{\frac{2}{3}} t^{2}-2 \varepsilon t^{3}+\mathcal{O}\left(t^{4}\right) .
$$

Equation (3.3) can be non-dimensionalized with the use of the initial separation, $R_{0}$, and the characteristic time scale for that separation, $t_{0}=\left(R_{0}^{2} / \varepsilon\right)^{1 / 3}$, leading to

$$
\frac{\left\langle\delta \mathbf{R}(t)^{2}\right\rangle}{R_{0}^{2}}=\frac{11}{3} C_{2}\left(\frac{t}{t_{0}}\right)^{2}-2\left(\frac{t}{t_{0}}\right)^{3}+\mathcal{O}\left(t^{4}\right) .
$$

At very short times, the first term on the right hand side in equation (3.4) dominates the separation of the particle pair. This term is equivalent to Batchelor scaling and, due to being quadratic in time, does not reveal any irreversibility of the flow. The next term in the expansion is the first to break time symmetry by having an odd power in time. This can be easily seen when explicitly rewriting equation (3.4) for the case of backward dispersion $(t \rightarrow-t)$

$$
\frac{\left\langle\delta \mathbf{R}(-t)^{2}\right\rangle}{R_{0}^{2}}=\frac{11}{3} C_{2}\left(\frac{t}{t_{0}}\right)^{2}+2\left(\frac{t}{t_{0}}\right)^{3}+\mathcal{O}\left(t^{4}\right) .
$$

Studying equation (3.2) again, one finds that the $t^{3}$ term arises from the fact that in a turbulent flow, the derivative of the squared relative velocity does not vanish, as it would be the case for a velocity field that is delta-correlated in time. Instead, for a pair separation in the inertial range and at high Reynolds numbers, the initial change in the squared relative velocity is fully determined by the rate of the energy flux through scales, $\varepsilon$. The first term to break time symmetry can thus be directly linked to the turbulence energy cascade. For a 3D turbulent flow, energy is transported from large to small scales, leading to an energy transfer rate $\varepsilon>0$. Equations (3.4) and (3.5) thus predict that the short-time particle separation forwards in time $(t>0)$ is diminished while the separation backwards in time $(t<0)$ is enhanced by the energy cascade in comparison to the case where $\varepsilon=0$.

It is interesting to note that all previous studies on the asymmetry between forward and backward dispersion also observed a faster separation backwards in time (Sawford 2001, Berg et al. 2006, Bragg et al. 2014). These studies were however concerned with the 
evolution at much larger times, corresponding to the Richardson regime, so that the obtained observations cannot be traced back to equation (3.5).

Aside from the time asymmetry, another interesting aspect of the short-time approach is the timespan for which the Taylor series remains valid. If all terms of the expansion in equation (3.4) could be written as functions of $t / t_{0}$ with prefactors of order one, as is the case for the first two terms, the Taylor series would converge for times $t<a t_{0}$, with $a$ also an order one constant. However, it can be shown that only terms with odd powers of time can be consequently written in this form. For the even terms, starting at $t^{4}, \tau_{\eta}$ is the dominant time-scale, leading to a severe reduction of the radius of convergence (Frishman 2013, a detailed proof is shown in appendix A.2). Due to the different convergence radii of the odd and even terms, I define the symmetric and antisymmetric functions

$$
\begin{aligned}
& F^{s}(t)=\frac{\left\langle\delta \mathbf{R}(t)^{2}+\delta \mathbf{R}(-t)^{2}\right\rangle}{2 R_{0}^{2}}=\frac{\left\langle\mathbf{V}(0)^{2}\right\rangle}{R_{0}^{2}} t^{2}+\mathcal{O}\left(t^{4}\right)=\frac{11}{3} C_{2}\left(\frac{t}{t_{0}}\right)^{2}+\mathcal{O}\left(t^{4}\right) \\
& F^{a}(t)=\frac{\left\langle\delta \mathbf{R}(t)^{2}-\delta \mathbf{R}(-t)^{2}\right\rangle}{2 R_{0}^{2}}=\frac{\langle\mathbf{V}(0) \cdot \mathbf{A}(0)\rangle}{R_{0}^{2}} t^{3}+\mathcal{O}\left(t^{5}\right)=-2\left(\frac{t}{t_{0}}\right)^{3}+\mathcal{O}\left(t^{5}\right) .
\end{aligned}
$$

Note that while $F^{a}(t)$ converges for $t \lesssim t_{0}$, the radius of convergence of $F^{s}(t)$ cannot be easily computed but is most probably of the order of $\tau_{\eta}$. Furthermore, only $F^{a}(t)$ contributes to the breaking of the time symmetry.

\subsection{Data Analysis}

In order to compute all relevant pair statistics from the recorded datasets, a $\mathrm{C}++$ code written by myself was used to successively analyze the particle tracks from all videos stored in one velocity-acceleration file (see appendix C.3). For each video, a nested loop identifies all particle trajectories with a temporal overlap (i.e. shared frames). If a pair with an overlap is found, the separation $|\mathbf{R}|$ between the two particles is computed for all shared frames. For each frame $k$ for which $R_{0}-\triangle R_{0} \leq|\mathbf{R}| \leq R_{0}+\triangle R_{0}$, with a fixed tolerance $\Delta R_{0}$, the pair statistics of all shared frames are stored with frame $k$ corresponding to $t=0$ (figure 3.1). In the case that more than one frame fulfills the condition $R_{0}-\triangle R_{0} \leq|\mathbf{R}| \leq R_{0}+\triangle R_{0}$, the statistics of the particle pair are stored several times, always with a different frame corresponding to $t=0$. This increases statistics while eliminating any possible bias due to the choice of frame $k$. This process is repeated until the final statistics contain the information of all possible trajectory pairs from all videos recorded for an experiment. The computed quantities are the mean squared relative separation (as well as some variations of it), the mean squared relative velocity, and the mean relative velocity-acceleration correlation, $\langle\delta \mathbf{u} \cdot \delta \mathbf{a}\rangle$.

The tolerance on the initial separation, $\triangle R_{0}$, is chosen to be $5 \%$ of the value of $R_{0}$ for all experimental datasets. Furthermore, in order to ensure that all shown results are statistically well converged, only datasets with more than $10^{7}$ observed pairs at $t=0$ are shown. The time evolution is only shown up to a time $t$ at which the number of observed pairs drops below $10^{6}$. 


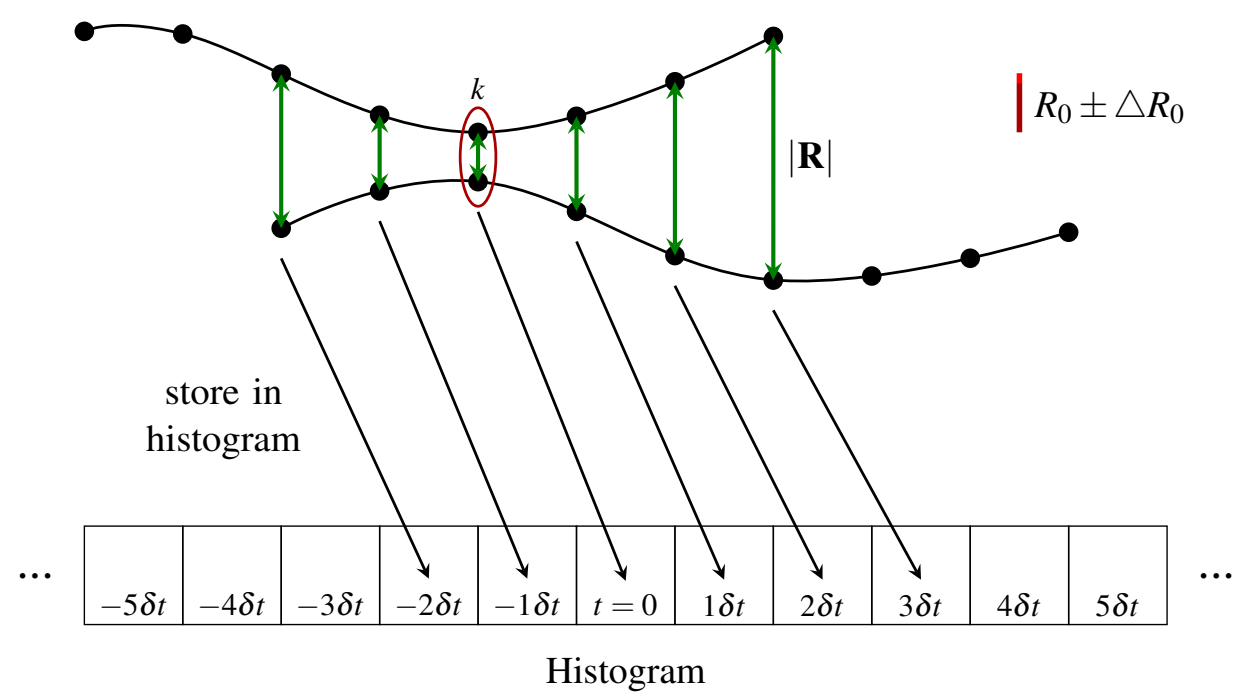

Figure 3.1.: Sketch of how each pair of trajectories is analyzed. The separation $|\mathbf{R}|$ is compared with $R_{0} \pm \triangle R_{0}$ for all temporally overlapping frames of the two trajectories. For each frame in which $|\mathbf{R}|$ falls into the specified range (here frame $k$ ), the pair statistics of all overlapping frames are stored in a histogram with frame $k$ defining $t=0$.

\subsection{Experimental Results}

Figure 3.2 shows the experimental results for $\left\langle\delta \mathbf{R}(t)^{2}\right\rangle$ and $\left\langle\delta \mathbf{R}(-t)^{2}\right\rangle$ plotted over $|t| / t_{0}$ and compensated by both $R_{0}^{2} \frac{11}{3} C_{2}\left(t / t_{0}\right)^{2}$, according to equation (3.4), and $\left\langle\mathbf{V}(0)^{2}\right\rangle t^{2}$, according to equation (3.1). Data at three different Reynolds numbers and several initial separations in the inertial range are shown. It can be noticed that in both cases the forward and backward dispersion show a plateau at a value near unity, after which the forward dispersion data drops below the backward dispersion data, exactly as predicted. While a collapse can be seen for the general case in figure 3.2 (b), the same is not true in figure 3.2 (a) for which the measured mean squared dispersion is compensated according to the formula for an isotropic flow. The value for $C_{2}$ seems not to be constant over the range of initial separations studied here and its real value is somewhat larger than the used value of $C_{2}=2.1$. This is due to the fact that the studied flow is not perfectly isotropic (see appendix B.1). Especially at the borders of the measurement volume, the mean flow has a non-negligible magnitude. It is therefore not surprising that the more general case in figure 3.2(b) leads to a much clearer collapse. Another observation that can be made from the good collapse in figure 3.2(b) is that $t_{0}$ seems indeed to be the correct scale for the time axis. Especially the good agreement among the different curves of the point in time at which they deviate from the plateau is a strong validation of this.

Although the results seem to agree well with the theoretical prediction, one major difference can be seen. While equation (3.5) predicts that the $t^{3}$ term leads to an enhancement of 


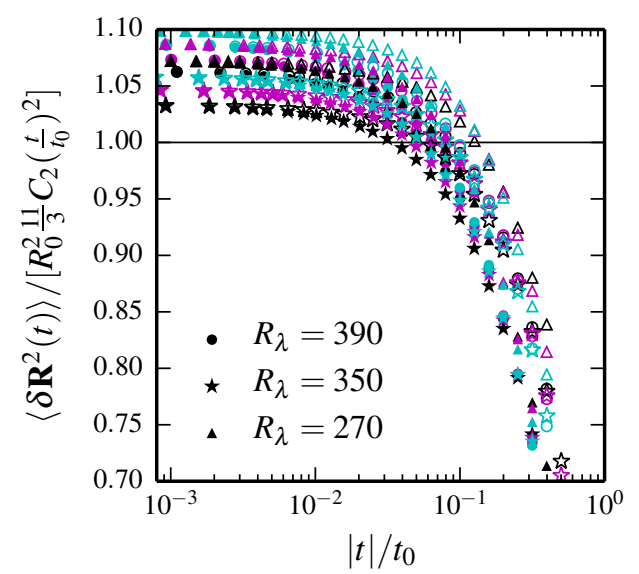

(a) Isotropy Assumed

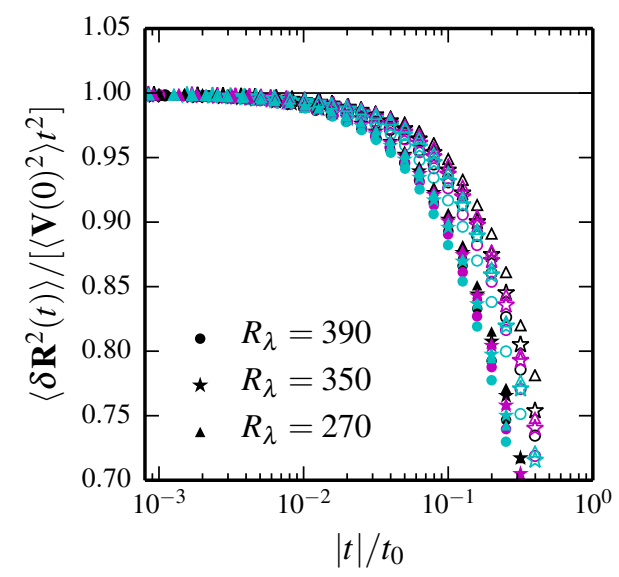

(b) General Case

Figure 3.2.: Mean squared separation $\left\langle\delta \mathbf{R}(t)^{2}\right\rangle / R_{0}^{2}$ forwards and backwards in time as a function of $|t| / t_{0}$. For $R_{\lambda}=390$, the initial separations are $241 \eta(\bullet), 276 \eta$ (•) and $310 \eta(\bullet)$. For $R_{\lambda}=350$, the initial separations are $212 \eta(\star), 242 \eta(\star)$ and $273 \eta(\star)$, and for $R_{\lambda}=270$, they are $135 \eta(\mathbf{\Delta}), 154 \eta(\Delta)$ and $173 \eta(\Delta)$. Filled and open symbols represent the results forwards $(t>0)$ and backwards in time $(t<0)$, respectively. (a) The mean squared separation is compensated assuming an isotropic flow with $C_{2}=2.1$. (b) The mean squared separation is compensated according to the more general equation (3.1).

backward dispersion over the $t^{2}$ behavior, figure 3.2(b) shows that the backward dispersion never actually rises above unity. This inconsistency with the theoretical prediction can be explained by a bias of the recorded data due the finite size of the measurement volume. A finite observation volume always entails a finite observation time of the studied tracer particles. This observation time is not equal for all particles because particles with a larger velocity cross the volume more quickly than slower particles do. The same holds true for particle pairs. A large relative velocity $\mathbf{V}(t)$ usually leads to a short observation time and vice versa $^{2}$. Consequently, the number of statistics varies with time in a biased manner, meaning that at later times only slowly separating particles remain. For an ensemble average over an arbitrary, time-dependent function, $\langle k(t)\rangle$, it thus matters whether the average is taken over all pairs observed at time $t$ or for example over those pairs observed at time $t^{\prime}>t$ as illustrated in figure 3.3. Taking this time dependence into account, such an ensemble average shall be written as $\langle\cdot\rangle_{t}$ in the following, where the subscript $t$ denotes the time at which the ensemble is chosen. Experimentally, the average of the mean squared separation at time $t$ is taken over all trajectories observed at time $t$, so that in the new notation equation (3.1) is

\footnotetext{
${ }^{2}$ There exists of course the case that a slowly separating particle pair starts very close to the boundary of the observation volume, thus also leading to a short observation time. The starting position at time $t=0$ is independent of the kinetic properties of the particle pair, however, and thus does not contribute to any form of bias.
} 


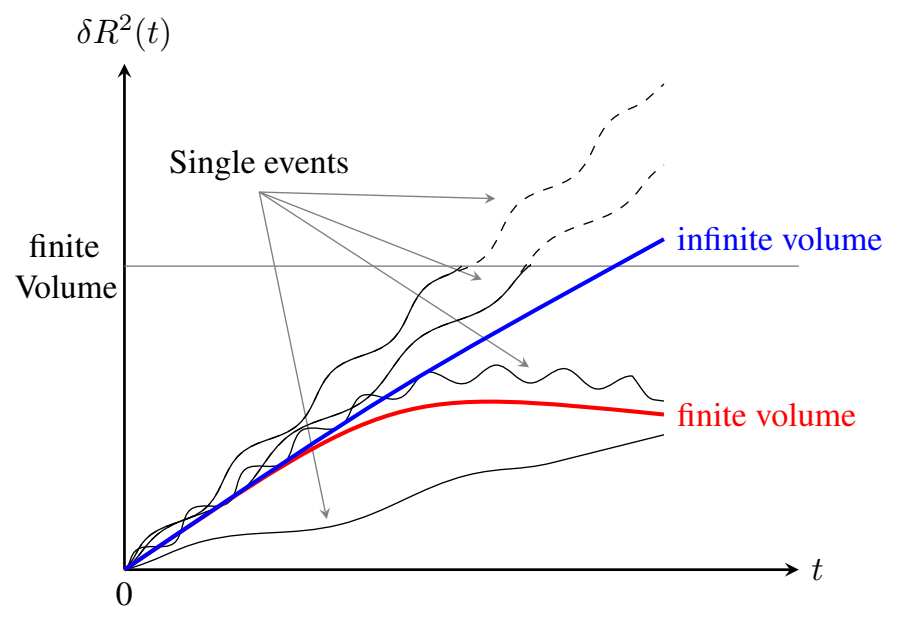

Figure 3.3.: Sketch of the effect of a finite measurement volume on the average over an ensemble of pair trajectories. Examples for individual pair trajectories are shown in black. The dashed part of the trajectory is not visible in the case of a finite observation volume. The blue and red line show the average over many pairs for the cases of an infinite and finite observation volume, respectively.

given by

$$
\left\langle\delta \mathbf{R}(t)^{2}\right\rangle_{t}=\left\langle\mathbf{V}(0)^{2}\right\rangle_{t} t^{2}+\langle\mathbf{V}(0) \cdot \mathbf{A}(0)\rangle_{t} t^{3}+\mathcal{O}\left(t^{4}\right) .
$$

It becomes directly clear that the right hand side is changed in a significant manner. If all pairs could be tracked indefinitely, the averages over the velocity and acceleration at time $t=0$ should be constant. In the case of a finite observation volume, however, these averages become functions of time, always corresponding to the ensemble of particle pairs observed at time $t$. One can explicitly capture this time dependence by defining

$$
\left\langle\delta \mathbf{R}(t)^{2}\right\rangle_{t}=\left\langle\mathbf{V}(0)^{2}\right\rangle_{0} f_{1}(t) t^{2}+\langle\mathbf{V}(0) \cdot \mathbf{A}(0)\rangle_{0} f_{2}(t) t^{3}+\mathcal{O}\left(t^{4}\right),
$$

with the bias functions $f_{1}(t)$ and $f_{2}(t)$ obeying $f_{1}(0)=f_{2}(0)=1$ by construction. The averages $\left\langle\mathbf{V}(0)^{2}\right\rangle_{0}$ and $\langle\mathbf{V}(0) \cdot \mathbf{A}(0)\rangle_{0}$ are taken over all pairs observed at $t=0$ and correspond to the case of an infinite measurement volume. The bias is thereby shifted completely into the functions $f_{i}(t)$. By following each individual particle pair for the same duration forwards and backwards in time, i.e. $t \in\left[-t_{1}, t_{1}\right]$, the bias functions can be made exactly time-symmetric, since at any time $t \geq 0$ one obtains the same ensemble of pairs as at time $-t$. For the experimental data presented in this thesis, it can be found that both bias functions decrease with time and that $0.9 \lesssim f_{i}(t) \lesssim 1.0$ for $|t|<0.1 t_{0}$, thus only leading to an error of up to $10 \%$ in each term (see figure 3.4). Since the first term in the expansion in equation (3.9) is itself much larger than the second term, a $10 \%$ error on the first term however fully obscures the effect of the second one. This is exactly what is observed in figure 3.2. Although the split between forward an backward dispersion due to the $t^{3}$ term can be observed, the enhancement of the backward dispersion above the $t^{2}$ behavior is masked by the decreasing value of $f_{1}(t)$. 

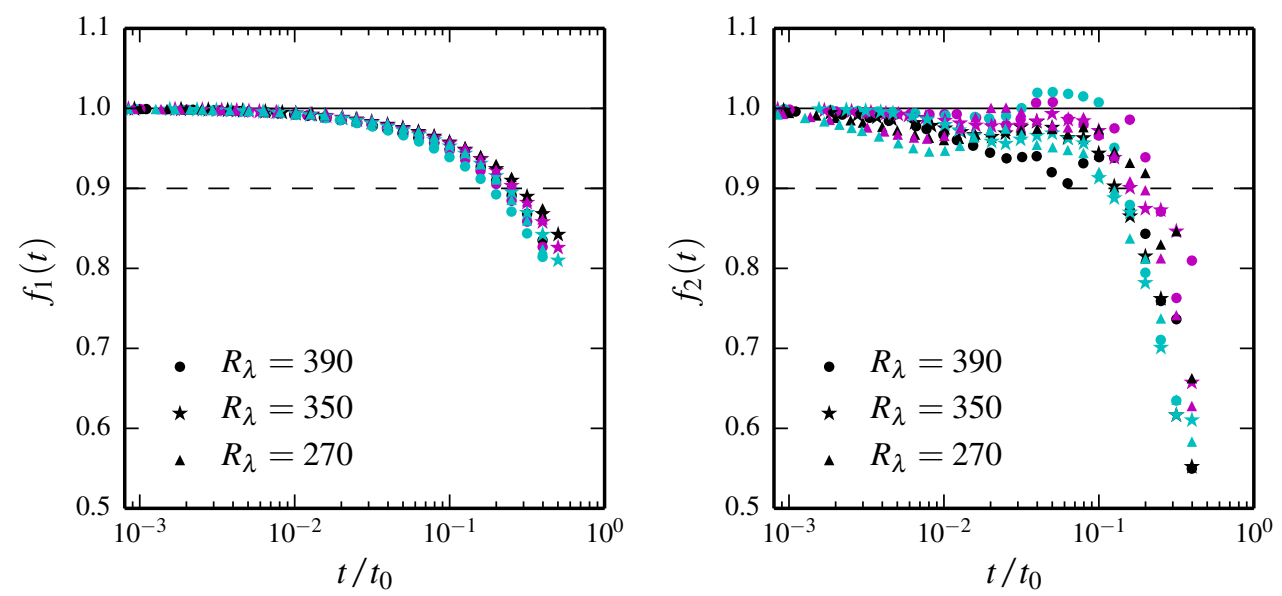

Figure 3.4.: Bias functions $f_{1}(t)$ and $f_{2}(t)$ as obtained from the time-dependent averages $\left\langle\mathbf{V}(0)^{2}\right\rangle_{t}$ and $\langle\mathbf{V}(0) \cdot \mathbf{A}(0)\rangle_{t}$, respectively. The horizontal dashed line indicates an influence of the bias functions of $10 \%$. Datasets and color code are identical to those shown in figure 3.2 on page 51.

One way to solve this problem is to subtract the $t^{2}$ term before averaging and then add an unbiased $t^{2}$ term according to

$$
\left\langle\delta \mathbf{R}(t)^{2}-\mathbf{V}(0)^{2} t^{2}\right\rangle_{t}+\left\langle\mathbf{V}(0)^{2}\right\rangle_{0} t^{2}=\left\langle\mathbf{V}(0)^{2}\right\rangle_{0} t^{2}+\langle\mathbf{V}(0) \cdot \mathbf{A}(0)\rangle_{0} f_{2}(t) t^{3}+\mathcal{O}\left(t^{4}\right) .
$$

The leading term on the right hand side thus remains unbiased, allowing the effect of the $t^{3}$ term to be visible. Figure 3.5 shows the mean squared separation corrected in this way, compensated by the squared relative velocity. One can nicely see how the backward dispersion is corrected towards higher values and even rises slightly above the plateau while the forward dispersion remains clearly below it, as it was predicted.

An even cleaner way of coping with the finite volume bias is to look directly at the symmetric and antisymmetric functions, $F_{t}^{s}(t)$ and $F_{t}^{a}(t)$, for a biased measurement:

$$
\begin{aligned}
F_{t}^{s}(t) & =\frac{\left\langle\delta \mathbf{R}(t)^{2}+\delta \mathbf{R}(-t)^{2}\right\rangle_{t}}{2 R_{0}^{2}}=\frac{\left\langle\mathbf{V}(0)^{2}\right\rangle_{0}}{R_{0}^{2}} f_{1}(t) t^{2}+\mathcal{O}\left(t^{4}\right) \\
\text { and } \quad F_{t}^{a}(t) & =\frac{\left\langle\delta \mathbf{R}(t)^{2}-\delta \mathbf{R}(-t)^{2}\right\rangle_{t}}{2 R_{0}^{2}}=\frac{\langle\mathbf{V}(0) \cdot \mathbf{A}(0)\rangle_{0}}{R_{0}^{2}} f_{2}(t) t^{3}+\mathcal{O}\left(t^{5}\right) \\
& =-2 f_{2}(t)\left(\frac{t}{t_{0}}\right)^{3}+\mathcal{O}\left(t^{5}\right) .
\end{aligned}
$$

Here, the time symmetry of the bias functions was used to ensure the full cancellation of the odd or even terms, respectively. Even though both $F_{t}^{s}(t)$ and $F_{t}^{a}(t)$ still experience the effect of the bias functions, $F_{t}^{a}(t)$ shows the symmetry-breaking $t^{3}$-term now at leading order with only a small diminishing effect of the bias function by less than $10 \%$ for $t<0.1 t_{0}$. Figure 3.6 


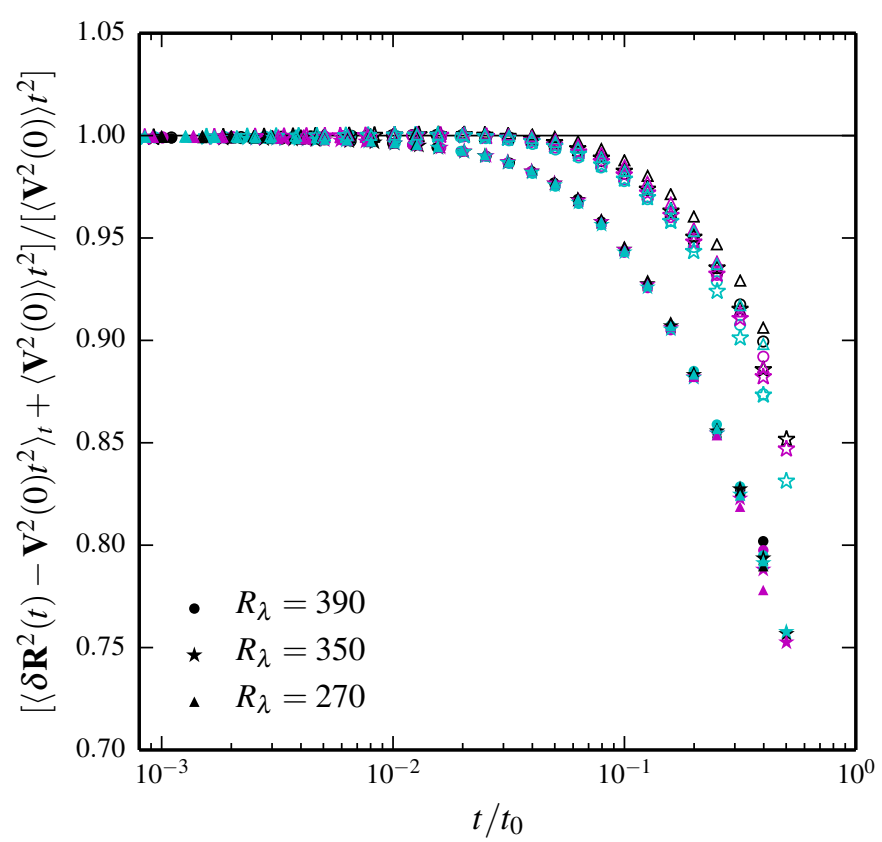

Figure 3.5.: Experimental results for the bias-corrected mean squared separation. Filled and open symbols represent forwards and backwards dispersion, respectively. Datasets and color code are identical to those shown in figure 3.2 on page 51 .

shows the asymmetric function, $F_{t}^{a}(t)$ compensated by $-2\left(t / t_{0}\right)^{3}$, for the same experimental data as above. All curves show plateaus up to $0.1 t_{0}$ and then drop together. The heights of the plateaus deviate less than $25 \%$ from unity. Taking into account the large $10 \%$ uncertainty of the energy transfer rate (measured for the small measurement volume), together with the even larger uncertainty of adopting this value for the large measurement volume, the results in figure 3.6 are in good agreement with the theoretical prediction.

For comparison, the mean squared change of separation was also computed from DNS data at a similar Reynolds number, $R_{\lambda}=300$, and a scale separation between largest and smallest scales of $L / \eta=250$. The DNS data was kindly provided by Alain Pumir from the École Normale Supérieure de Lyon and was also published in Jucha et al. (2014). For the DNS, the Navier-Stokes equations were solved directly with a standard pseudo-spectral code (described e.g. in Voßkuhle et al. 2013) for periodic boundary conditions. More than $3 \cdot 10^{4}$ fluid particle trajectories were processed. Figure 3.7 shows a comparison of the experimental and numerical results for the compensated $F_{t}^{a}(t)$. Due to the strong uncertainties of the energy transfer rates, the directly measured value of $\langle\mathbf{V}(0) \cdot \mathbf{A}(0)\rangle_{0}$ is used for compensation. Very good agreement between the experimental and numerical data can be found. Only at larger times, $t \gtrsim 0.2 t_{0}$, the experimental data drops slightly faster than the numerical data. This difference may be attributed to the residual finite volume bias that only affects the experimental but not the DNS data. All data sets show a rapid decrease towards zero 


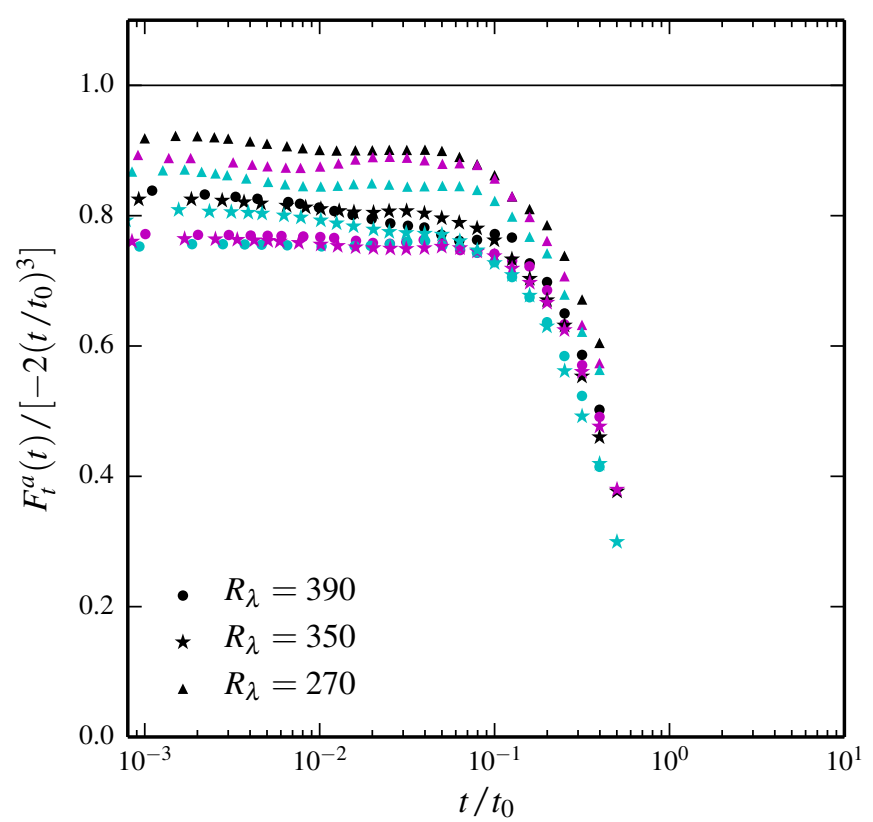

Figure 3.6.: Experimental results for the odd terms of the mean squared separation, compensated to yield a plateau with a height of one. Datasets and color code are identical to those shown in figure 3.2 on page 51 .

at sufficiently long times. This is the expected behavior, since the two particles forming the pair become uncorrelated over time and no difference between forward and backward dispersion should remain. For intermediate times, however, a Richardson-like scaling is expected (Sawford 2001, Berg et al. 2006, Bragg et al. 2014). Following equation (1.29), the difference between forward and backward dispersion in a Richardson-scaling regime is given by

$$
F_{t}^{a}(t)=\frac{\left\langle\delta \mathbf{R}(t)^{2}-\delta \mathbf{R}(-t)^{2}\right\rangle_{t}}{2 R_{0}^{2}} \stackrel{\text { Richardson }}{=} \frac{1}{2}\left(g_{f}-g_{b}\right)\left(\frac{t}{t_{0}}\right)^{3},
$$

where a value of $g_{f}-g_{b} \approx-0.6$ has been proposed by Berg et al. (2006) based on measurements at $R_{\lambda}=170$. In figure 3.7, another plateau with a height of $-\frac{1}{4}\left(g_{f}-g_{b}\right) \approx 0.15$ should thus be visible, where I used the fact that $\langle\mathbf{V}(0) \cdot \mathbf{A}(0)\rangle_{0}=-2 \varepsilon$. The numerical data clearly drops below this point without any indication of a plateau. The experimental data shown here does not reach that far since only times with more than $10^{6}$ observed pairs are shown. Lowering this very strict cut-off to $10^{4}$, a very rapid drop below 0.15 with no indication of a plateau is revealed (see appendix A.3). Even though a plateau at $-\frac{1}{4}\left(g_{f}-g_{b}\right) \approx 0.15$ is not observed, it cannot be ruled out that a plateau for a much smaller value of $\left(g_{f}-g_{b}\right)$ might exist. This result will be discussed in greater detail in chapter 6 .

Thus far, I found that the intrinsic time irreversibility of turbulent flows can be tested by exploring the mean squared change of separation of a pair of tracer particles. Focusing on 


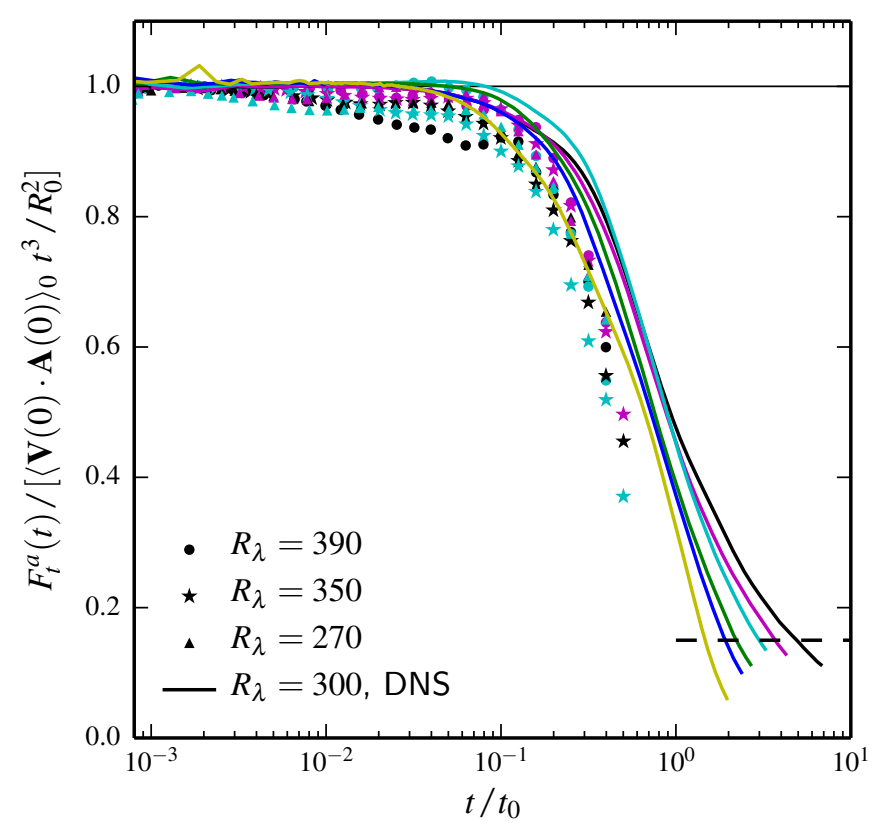

Figure 3.7.: Experimental and numerical results for the odd terms of the compensated mean squared change of separation. Datasets and color code for the experimental data are identical to those shown in figure 3.2 on page 51. For the DNS data at $R_{\lambda}=300$, the initial separations are $19 \eta(-), 38 \eta(-), 58 \eta(-), 77 \eta(-), 92 \eta(-)$ and $123 \eta$ (-). The dashed horizontal line indicates the prediction by Richardson scaling as proposed by Berg et al. (2006).

the short-time behavior, I confirmed that pair-dispersion is faster backwards than forwards in time and I showed that this asymmetry is directly connected to the directionality of the turbulence energy cascade. It can be first seen for the $t^{3}$ term and, if properly normalized, is fully determined by the value of the energy transfer rate.

Although the results presented in this chapter are precise and significant, the effect of the symmetry breaking is only small. In the next chapter, I will discuss a much stronger manifestation of the intrinsic time irreversibility of turbulence by analyzing the shape deformation of clusters of four particles. I will show that the symmetry-breaking term there appears at first order in time and thus has a much larger effect. 


\section{CHAPTER 4}

\section{Four-Particle Dispersion}

In this chapter, I analyze the effect of the intrinsic time irreversibility of turbulent flows on the shape deformation of clusters of four particles. In analogy to chapter 3, I perform a short-time expansion for the size and shape properties of the cluster in section 4.1. In section 4.2, I briefly explain how the recorded videos are evaluated and I present the experimental results in section 4.3. Again, a comparison with DNS data provided by Alain Pumir from École Normale Supérieure de Lyon will be shown.

\subsection{Short-Time Expansion}

Following section 1.3.4, the shape tensor for a cluster of four particles, a tetrahedron, is given by

$$
G(t)_{i j}=\sum_{a=1}^{3} \rho_{i}^{(a)}(t) \rho_{j}^{(a)}(t),
$$

with the form of the $\rho$-vectors, $\rho^{(a)}(t)$, specified in equation (1.35). The shape tensor is fully specified by its three eigenvalues, $g_{i}(t)$, and three Euler angles. No easy relation exists to obtain the Euler angles, but the eigenvalues, sorted by size such that $g_{1} \geq g_{2} \geq g_{3}$, suffice for a classification of the shape of the tetrahedron (see section 1.3.4). Furthermore, the size of the tetrahedron is described by the trace of the shape tensor in the form of the radius of gyration as given in equation (1.34).

In order to study the evolution of the shape tensor, an equation of motion needs to be derived. Introducing the velocities $\mathbf{v}^{(a)}(t)=\frac{\mathrm{d}}{\mathrm{d} t} \rho^{(a)}(t)$ as the change of the $\rho$-vectors with 
time, and taking the time derivative of equation (4.1), one finds

$$
\begin{aligned}
\frac{\mathrm{d}}{\mathrm{d} t} G(t)_{i j} & =\sum_{a=1}^{3} \rho_{i}^{(a)}(t) v_{j}^{(a)}(t)+\sum_{a=1}^{3} v_{i}^{(a)}(t) \rho_{j}^{(a)}(t) \\
& =\sum_{a=1}^{3} \rho_{i}^{(a)}(t)\left[\sum_{k=1}^{3} \rho_{k}^{(a)}(t) M_{k j}(t)\right]+\sum_{a=1}^{3}\left[\sum_{k=1}^{3} \rho_{k}^{(a)}(t) M_{k i}(t)\right] \rho_{j}^{(a)}(t) .
\end{aligned}
$$

At the second equality, the definition of the perceived velocity gradient tensor, $\mathbf{v}^{(a)}(t)=$ $\rho^{(a)}(t)^{T} M(t)$, from equation (1.36) was used. Reinserting equation (4.1), one finally obtains

$$
\frac{\mathrm{d}}{\mathrm{d} t} \mathbf{G}(t)=\mathbf{G}(t) \cdot \mathbf{M}(t)+\mathbf{M}^{T}(t) \cdot \mathbf{G}(t) .
$$

This equation of motion links the shape evolution of a tetrahedron to the surrounding flow field. It can be solved for short times by expanding both the shape tensor and the perceived velocity gradient tensor around $t=0$ with

$$
\begin{aligned}
& \mathbf{G}(t)=\mathbf{G}_{0}+\mathbf{G}_{1} t+\mathbf{G}_{2} \frac{t^{2}}{2}+\mathbf{G}_{3} \frac{t^{3}}{6}+\mathcal{O}\left(t^{4}\right), \\
& \mathbf{M}(t)=\mathbf{M}_{0}+\mathbf{M}_{1} t+\mathbf{M}_{2} \frac{t^{2}}{2}+\mathbf{M}_{3} \frac{t^{3}}{6}+\mathcal{O}\left(t^{4}\right),
\end{aligned}
$$

where $\mathbf{G}_{n}=\left.\frac{\mathrm{d}}{\mathrm{d} t} G(t)\right|_{t=0}$ and $\mathbf{M}_{n}=\left.\frac{\mathrm{d}}{\mathrm{d} t} M(t)\right|_{t=0}$. As an initial condition, an isotropic tetrahedron with edge length $l$ is assumed, so that $\mathbf{G}(0)=\mathbf{G}_{0}=\frac{l^{2}}{2} \mathbb{1}$. Inserting the above Taylor series into equation (4.4) and comparing the left- and right-hand side for each order in $t$, one finds

$$
\begin{aligned}
\mathbf{G}_{0}= & \frac{l^{2}}{2} \mathbb{1} \quad \text { (initial condition), } \\
\mathbf{G}_{1}= & \mathbf{M}_{0}^{T} \mathbf{G}_{0}+\mathbf{G}_{0} \mathbf{M}_{0}=\frac{l^{2}}{2}\left(\mathbf{M}_{0}^{T}+\mathbf{M}_{0}\right), \\
\mathbf{G}_{2}= & \mathbf{M}_{1}^{T} \mathbf{G}_{0}+\mathbf{G}_{0} \mathbf{M}_{1}+\mathbf{M}_{0}^{T} \mathbf{G}_{1}+\mathbf{G}_{1} \mathbf{M}_{0} \\
= & \frac{l^{2}}{2}\left(\mathbf{M}_{1}^{T}+\mathbf{M}_{1}+\left(\mathbf{M}_{0}^{T}\right)^{2}+2 \mathbf{M}_{0}^{T} \mathbf{M}_{0}+\mathbf{M}_{0}^{2}\right), \\
\mathbf{G}_{3}= & \mathbf{M}_{2}^{T} \mathbf{G}_{0}+\mathbf{G}_{0} \mathbf{M}_{2}+2 \mathbf{M}_{1}^{T} \mathbf{G}_{1}+2 \mathbf{G}_{1} \mathbf{M}_{1}+\mathbf{M}_{0}^{T} \mathbf{G}_{2}+\mathbf{G}_{2} \mathbf{M}_{0} \\
= & \frac{l^{2}}{2}\left(\mathbf{M}_{2}^{T}+\mathbf{M}_{2}+\left(\mathbf{M}_{0}^{T}\right)^{3}+3\left(\mathbf{M}_{0}^{T}\right)^{2} \mathbf{M}_{0}+3 \mathbf{M}_{0}^{T} \mathbf{M}_{0}^{2}+\mathbf{M}_{0}^{3}+2 \mathbf{M}_{1}^{T} \mathbf{M}_{0}^{T}\right. \\
& \left.\quad+\mathbf{M}_{0}^{T} \mathbf{M}_{1}^{T}+2 \mathbf{M}_{0} \mathbf{M}_{1}+\mathbf{M}_{1} \mathbf{M}_{0}+3 \mathbf{M}_{1}^{T} \mathbf{M}_{0}+3 \mathbf{M}_{0}^{T} \mathbf{M}_{1}\right) .
\end{aligned}
$$

One can further simplify the above results by using the perceived rate of strain tensor, $\mathbf{S}(t)=\frac{1}{2}\left(\mathbf{M}(t)+\mathbf{M}^{T}(t)\right)$, and the perceived rate of rotation tensor, $\Omega(t)=\frac{1}{2}\left(\mathbf{M}(t)-\mathbf{M}^{T}(t)\right)$. Expanding both around $t=0$, the $n$th derivatives at $t=0$ are then given by $\mathbf{S}_{n}=\frac{1}{2}\left(\mathbf{M}_{n}+\mathbf{M}_{n}^{T}\right)$ and $\Omega_{n}=\frac{1}{2}\left(\mathbf{M}_{n}-\mathbf{M}_{n}^{T}\right)$, respectively. The short-time evolution of the shape tensor can then 
be written as

$$
\begin{aligned}
\mathbf{G}(t)= & \frac{l^{2}}{2}\left(\mathbb{1}+2 \mathbf{S}_{0} t+\left(2 \mathbf{S}_{1}+4 \mathbf{S}_{0}^{2}+2\left[\mathbf{S}_{0}, \Omega_{0}\right]\right) t^{2}+\left(2 \mathbf{S}_{2}+8 \mathbf{S}_{0}^{3}+6\left[\mathbf{S}_{0}^{2}, \Omega_{0}\right]\right.\right. \\
& \left.\left.+2\left[\left[\mathbf{S}_{0}, \Omega_{0}\right], \Omega_{0}\right]+6 \mathbf{S}_{0} \cdot \mathbf{S}_{1}+6 \mathbf{S}_{1} \cdot \mathbf{S}_{0}+2\left[\mathbf{S}_{1}, \Omega_{0}\right]+4\left[\mathbf{S}_{0}, \Omega_{1}\right]\right) t^{3}+\mathcal{O}\left(t^{4}\right)\right) .
\end{aligned}
$$

Here, I introduced the commutator $[\mathbf{A}, \mathbf{B}]=\mathbf{A} \cdot \mathbf{B}-\mathbf{B} \cdot \mathbf{A}$, and made use of the fact that the perceived rate of strain tensor is symmetric, $\mathbf{S}_{n}^{T}=\mathbf{S}_{n}$, while the perceived rate of rotation tensor is antisymmetric, $\Omega_{n}^{T}=-\Omega_{n}$.

Now, the size of the tetrahedron, expressed by the radius of gyration, can be easily obtained by taking the trace of the shape tensor. With equation (4.11), one finds

$$
\left\langle\mathbf{R}^{2}(t)\right\rangle=\langle\operatorname{tr}(\mathbf{G}(t))\rangle=\frac{l^{2}}{2}\left(3+2\left\langle\operatorname{tr}\left(\mathbf{S}_{0}^{2}\right)\right\rangle t^{2}+\left(\frac{4}{3}\left\langle\operatorname{tr}\left(\mathbf{S}_{0}^{3}\right)\right\rangle+2\left\langle\operatorname{tr}\left(\mathbf{S}_{0} \cdot \mathbf{S}_{1}\right)\right\rangle\right) t^{3}+\mathcal{O}\left(t^{4}\right)\right) .
$$

This result can be obtained by keeping in mind that the diagonal elements of commutators are always zero. Furthermore, I used the circumstance that, on average, the perceived rate of strain tensor is traceless, $\left\langle\operatorname{tr}\left(\mathbf{S}_{i}\right)\right\rangle \approx 0$, even though this is not true for each single realization. This can be understood when first thinking of the real velocity gradient tensor, $A_{i j}(\mathbf{x}, t)=\frac{\partial u_{i}(\mathbf{x}, t)}{\partial x_{j}}$, whose trace is zero due to the incompressibility of the flow, $\sum_{i} A_{i i}(\mathbf{x}, t)=$ $\nabla \cdot \mathbf{u}(\mathbf{x}, t)=0$. The same is then of course also true for the real rate of strain tensor since it is defined as the sum of the velocity gradient tensor and its transpose. It is important to note here that if a tensor is traceless at all times, it directly follows that all its time derivatives are traceless as well. One can now imagine a perceived rate of strain tensor which is defined over all points of the surface of a sphere. Such a tensor is clearly traceless as well because the volume of the sphere is conserved and the incompressibility argument still applies. For a perceived rate of strain tensor defined over only four points forming a tetrahedron, on the other hand, the trace is not always zero since the volume of the tetrahedron is not conserved. If one averages over many regular tetrahedra with the same size, however, the corner points of the tetrahedra sample the surface of a sphere, thus approximating the case above. For sufficiently many realizations, the average $\left\langle\operatorname{tr}\left(\mathbf{S}_{0}\right)\right\rangle$ thus vanishes. Assuming that the same holds true for a very short time range around zero in which the tetrahedra remain basically undeformed, $|t| \lesssim \tau_{\eta}$, it directly follows that the derivatives of the strain tensor at $t=0$, $\left\langle\operatorname{tr}\left(\mathbf{S}_{i}\right)\right\rangle$, are traceless too.

Aside from the approach using the shape tensor, the radius of gyration can also be expressed directly in terms of particle velocities and accelerations, similar to chapter 3 . With $\mathbf{X}_{\mathrm{com}}(t)=\frac{1}{4} \sum_{m=1}^{4} \mathbf{X}\left(t \mid \mathbf{y}^{(m)}, 0\right)$, the definition for the radius of gyration in equation (1.34) can be rewritten as

$$
\mathbf{R}^{2}(t)=\frac{1}{4} \sum_{a=1}^{4} \sum_{b \neq a}^{4}\left|\mathbf{X}\left(t \mid \mathbf{y}^{(a)}, 0\right)-\mathbf{X}\left(t \mid \mathbf{y}^{(b)}, 0\right)\right|^{2},
$$

which is just the average over the edge lengths of the tetrahedron. For an initially regular tetrahedron, with all edges of the length $l$, an average over sufficiently many tetrahedra then 
leads to

$$
\left\langle\mathbf{R}^{2}(t)\right\rangle=\frac{6}{4}\left\langle\mathbf{R}_{\text {pair }}^{2}(t)\right\rangle,
$$

where $\left\langle\mathbf{R}_{\text {pair }}^{2}(t)\right\rangle$ is the mean squared separation between two particles with an initial separation of $l$. The above result was obtained by noting that all six edges can be interpreted as individual pairs of particles, all with the same statistical properties and initial conditions. This implies that studying the size evolution of tetrahedra does not provide more information than studying pair dispersion does.

Much more promising is the study of the shape deformation of the volume spanned by the tetrahedron since it is intrinsic to $3 \mathrm{D}$ objects and cannot be observed for two-particle statistics. As discussed earlier, the shape of a tetrahedron can be well characterized by the eigenvalues $g_{i}$ of the shape tensor. In order to obtain those, the shape tensor needs to be diagonalized. Since $\mathbf{G}(t)$ is symmetric and real, there always exists a real rotation matrix $\mathbf{U}(t)$, such that

$$
\mathbf{G}^{D}(t)=\mathbf{U}(t) \cdot \mathbf{G}(t) \cdot \mathbf{U}^{T}(t)
$$

is diagonal and $G_{i i}^{D}(t)=g_{i}(t)$. All real rotation matrices are orthogonal, so that $\mathbf{U}(t) \cdot \mathbf{U}^{T}(t)=$ $\mathbb{1}$ and thus $\frac{\mathrm{d}}{\mathrm{d} t} \mathbf{U}(t)=\mathbf{R}(t) \cdot \mathbf{U}(t)$, with a real antisymmetric matrix $\mathbf{R}(t)$ for which $\mathbf{R}^{T}(t)=$ $-\mathbf{R}(t)$. Similarly to the shape tensor, one can thus expand the rotation matrix $\mathbf{U}(t)$ in a Taylor series around $t=0$ and express it through the antisymmetric matrix $\mathbf{R}(t)$ with

$$
\mathbf{U}(t)=\left[\mathbb{1}+\mathbf{R}_{0} t+\left(\mathbf{R}_{1}+\mathbf{R}_{0}^{2}\right) \frac{t^{2}}{2}+\left(\mathbf{R}_{2}+2 \mathbf{R}_{1} \cdot \mathbf{R}_{0}+\mathbf{R}_{0} \cdot \mathbf{R}_{1}+\mathbf{R}_{0}^{3}\right) \frac{t^{3}}{6}+\mathcal{O}\left(t^{4}\right)\right] \mathbf{U}(0) .
$$

Here, $\mathbf{R}_{n}=\left.\frac{\mathrm{d}^{n}}{\mathrm{~d} t^{n}} \mathbf{R}(t)\right|_{t=0}$ with $\mathbf{R}_{n}^{T}=-\mathbf{R}_{n}$ for all $n$. One can now use equation (4.15) to obtain the diagonalized shape tensor. After some lengthy calculation, see appendix A.4, one finds

$$
\begin{aligned}
\mathbf{G}^{D}(t)=\frac{l^{2}}{2}(\mathbb{1}+ & 2 \mathbf{S}_{0} t+\left(2 \mathcal{D}\left(\mathbf{S}_{1}\right)+4 \mathbf{S}_{0}^{2}\right) \frac{t^{2}}{2}+\left(2 \mathcal{D}\left(\mathbf{S}_{2}\right)+8 \mathbf{S}_{0}^{3}+12 \mathcal{D}\left(\mathbf{S}_{0} \cdot \mathbf{S}_{1}\right)\right. \\
+ & \left.\left.4 \mathcal{D}\left(\mathbf{R}_{0}^{2} \cdot \mathbf{S}_{0}\right)-4 \mathcal{D}\left(\mathbf{R}_{0} \cdot \mathbf{S}_{0} \cdot \mathbf{R}_{0}\right)+8 \mathcal{D}\left(\mathbf{R}_{0} \cdot \mathbf{S}_{1}\right)\right) \frac{t^{3}}{6}+\mathcal{O}\left(t^{4}\right)\right)
\end{aligned}
$$

Here, $\mathcal{D}(\mathbf{X})$ indicates that only the diagonal elements of the matrix $\mathbf{X}$ are used. Furthermore, all matrices in equation (4.17) are in the eigenframe of the perceived rate of strain tensor at $t=0, \mathbf{S}_{0}$. Denoting the eigenvalues of $\mathbf{S}_{0}$ with $s_{i}$, sorted such that $s_{1} \geq s_{2} \geq s_{3}$, the eigenvalues of the shape tensor are given by

$$
\begin{aligned}
\left\langle g_{i}(t)\right\rangle=\frac{l^{2}}{2}\left(1+2\left\langle s_{i}\right\rangle t\right. & +\left\langle S_{1, i i}+2 s_{i}^{2}\right\rangle t^{2}+\left\langle\frac{1}{3} S_{2, i i}+\frac{4}{3} s_{i}^{3}+2\left(\mathbf{S}_{0} \cdot \mathbf{S}_{1}\right)_{i i}\right. \\
+ & \left.\left.\frac{2}{3}\left(\mathbf{R}_{0}^{2} \cdot \mathbf{S}_{0}\right)_{i i}-\frac{2}{3}\left(\mathbf{R}_{0} \cdot \mathbf{S}_{0} \cdot \mathbf{R}_{0}\right)_{i i}+\frac{4}{3}\left(\mathbf{R}_{0} \cdot \mathbf{S}_{1}\right)_{i i}\right\rangle t^{3}+\mathcal{O}\left(t^{4}\right)\right) .
\end{aligned}
$$

It was discussed in section 1.3.4, that the eigenvalues of the perceived rate of strain tensor behave similarly to the eigenvalues of the real rate of strain tensor, in the sense that $\left\langle s_{1}\right\rangle>$ $\left\langle s_{2}\right\rangle>0>\left\langle s_{3}\right\rangle$ (Lüthi et al. 2007, Xu et al. 2008, Pumir et al. 2013). 


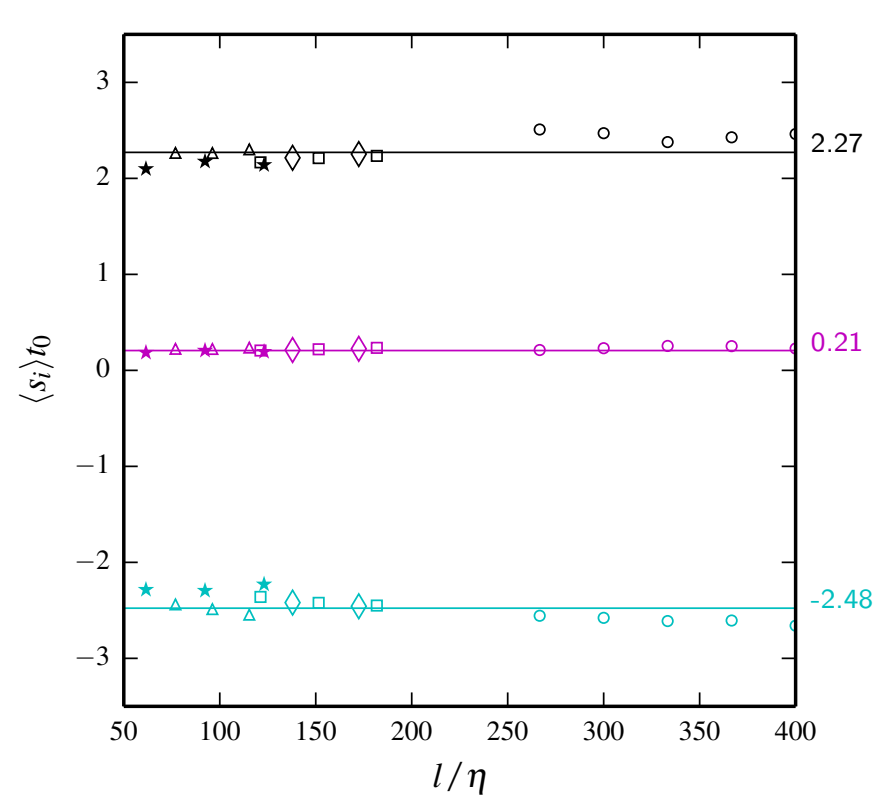

Figure 4.1.: average eigenvalues of the perceived rate of strain tensor, $\left\langle s_{i}\right\rangle$, as obtained from a regular tetrahedron with edge length $l$. The eigenvalues are non-dimensionalized with the characteristic time scale of an eddy of size $l, t_{0}=\left(l^{2} / \varepsilon\right)^{1 / 3}$. Open symbols represent experimental data sets with Reynolds numbers $R_{\lambda}=690$ (०), $R_{\lambda}=390(\diamond)$, $R_{\lambda}=350(\square)$, and $R_{\lambda}=270(\triangle)$. DNS data at $R_{\lambda}=300(\star)$ is shown as well (see section 3.3 for details).

Figure 4.1 shows the averaged eigenvalues of the perceived rate of strain tensor, $\left\langle s_{i}\right\rangle$, as obtained from the data presented in this thesis. It can be seen that the intermediate eigenvalue is indeed positive, and furthermore, that when the eigenvalues are non-dimensionalized with $t_{0}=\left(l^{2} / \varepsilon\right)^{1 / 3}$, they seem to be independent of Reynolds number and initial edge length of the tetrahedron. Therefore, mean values over all datasets are obtained with the additional requirement that the perceived rate of strain tensor is traceless on average. Most of the fluctuation of the points around the mean values can be attributed to the large uncertainties of $\varepsilon$ and $l$, introduced through $t_{0}$. The fact that the intermediate eigenvalue, $\left\langle s_{2}\right\rangle$, is nonzero leads to a strong time asymmetry of the evolution of the eigenvalues $g_{i}(t)$, as will be discussed in detail in section 4.3.

\subsection{Data Analysis}

The starting point for the analysis of the recorded videos with respect to tetrahedra evolution is again the velocity-acceleration file in which the trajectories of all recoded videos of one measurement are stored (see appendix C.3). A C++ code written by Haitao Xu is used to search for tetrahedra that, at least at one point in time, are regular with a prescribed edge length $l$. This searching algorithm is analogous to the one described in section 3.2 with 
the sole difference that each possible set of four, instead of two, particles is tested. As a consequence, the time needed to search for tetrahedra is proportional to $N^{4}$, where $N$ is the number of particles in one frame. Because this search algorithm is very time-consuming, it is performed in an isolated program and the found tetrahedra trajectories, the trajectories of the four particles spanning a tetrahedron, are stored into binary files. The corresponding file format is given in appendix C.4, where each file contains all tetrahedra trajectories of one recorded video.

These tetrahedra files are then processed by two evaluation programs written by myself, one to compute relevant quantities concerning the shape deformation and one to compute statistics of the perceived velocity gradient tensor. The shape deformation quantities are computed for the large measurement volume experiments due to their longer observation time, and the statistics of the perceived velocity gradient tensor are obtained from the small measurement volume experiments due to their higher spatial resolution.

Some of the large measurement volume datasets presented so far do not contain enough tetrahedra trajectories for sufficient statistical resolution. Displaying only datasets with more than $10^{5}$ observed tetrahedra at $t=0$, the remaining datasets are $R_{\lambda}=350(l=273 \eta)$ and $R_{\lambda}=270(l=135 \eta, 154 \eta$, and $135 \eta)$. Therefore, the already existing dataset at $R_{\lambda}=690$ ( $l=364 \eta, 424 \eta$, and $485 \eta$ ) will be used as well in order to replace the $R_{\lambda}=390$ dataset. The number of observed tetrahedra drops quickly with time, however, so only points with more than $5 \cdot 10^{4}$ observed tetrahedra are shown. Furthermore, the tetrahedra trajectories will not be symmetrized around $t=0$, as was done for the pair statistics. This means that each trajectory can be seen for a different number of frames forwards and backwards in time, thus increasing the number of statistics at larger times significantly. As a drawback, the finite volume bias is not time-symmetric anymore. For the small measurement volume datasets, all datasets show sufficient statistical convergence and can be used.

In order to analyze the shape deformation, the shape tensor is first computed according to equation (4.1) for every tetrahedron and every frame. If the determinant of the shape tensor is larger than a chosen minimal value, $\operatorname{det}(\mathbf{G}(t)) \geq 5 \cdot 10^{-5}$, a singular value decomposition is used to obtain the eigenvalues. The minimal value of the determinant is required to ensure the convergence of the algorithm for the singular value decomposition. The eigenvalues are sorted by size according to $g_{1} \geq g_{2} \geq g_{3}$ for each single frame. Further computed quantities are the radius of gyration, the mean squared change of separation as well as the mean squared relative velocity.

For the statistics of the perceived velocity gradient tensor, the procedure is slightly more complicated because all matrices have to be transformed into the eigenframe of $\mathbf{S}_{0}$. In a first loop through all frames of one tetrahedron trajectory, the perceived velocity gradient tensor is computed for every frame by means of equation (1.36), using a singular value decomposition with the requirement that $\operatorname{det}(\mathbf{M}(t)) \geq 5 \cdot 10^{-5}$. Furthermore, the perceived rate of strain and rate of rotation tensor are obtained as the symmetric and antisymmetric part of the perceived velocity gradient tensor. The eigenvalues and eigenvectors of the perceived rate of strain tensor, $\mathbf{S}(t)$, are computed using a Householder reduction. In a second loop through all frames, the just computed eigenvectors of $\mathbf{S}(0)=\mathbf{S}_{0}$ are used to transform $\mathbf{S}(t)$ and $\Omega(t)$ into the correct eigenframe. Finally, a histogram is generated from all tetrahedra trajectories of one measurement for the following quantities: $\left\langle s_{i}\right\rangle,\left\langle s_{i}^{2}\right\rangle,\left\langle s_{i}^{3}\right\rangle,\left\langle\mathcal{D}\left(\mathbf{S}_{1}\right)_{i}\right\rangle,\left\langle\mathcal{D}\left(\mathbf{S}_{0} \cdot \mathbf{S}_{1}\right)_{i}\right\rangle$ and 


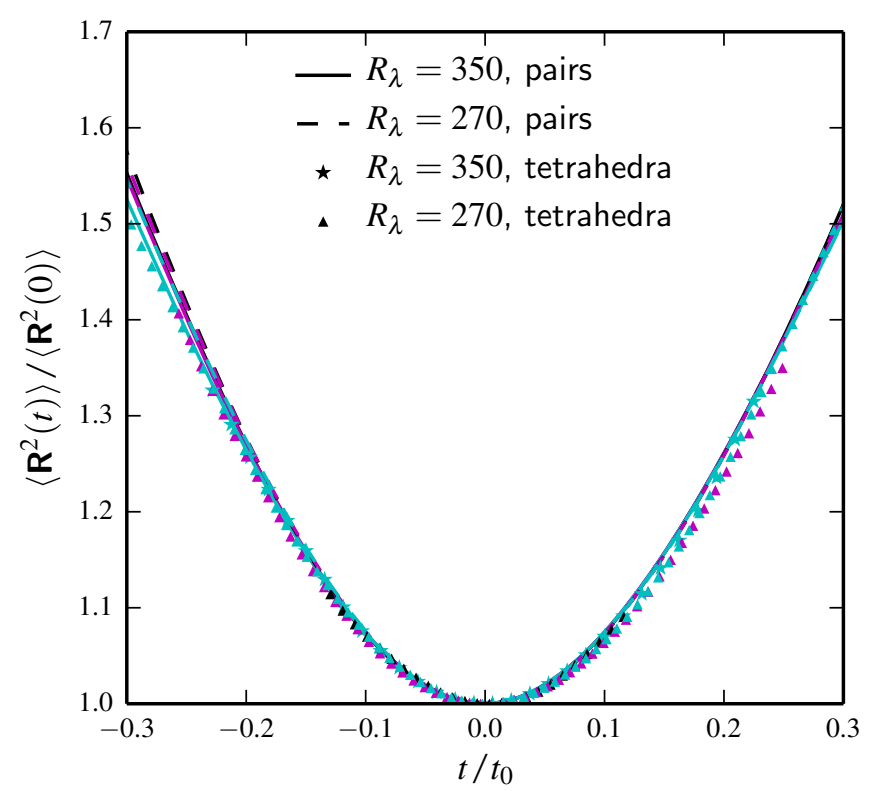

Figure 4.2.: The average radius of gyration computed for tetrahedra (filled symbols) and pairs (lines). For $R_{\lambda}=350$, the initial edge lengths $l$, or respectively the initial separations $R_{0}$, are $212 \eta$ (一, only for pairs), $242 \eta$ (一, only for pairs), and $273 \eta$ (一, $\star$ ). For $R_{\lambda}=270$, the initial edge lengths and separations are $135 \eta(--, \mathbf{\Lambda}), 154 \eta(--, \mathbf{\Delta})$, and $173 \eta(--, \Delta)$.

several traces of interest. For the time derivative, $\mathbf{S}_{1}$, a Gaussian filter with width $\tau_{\eta}$ and length $3 \tau_{\eta}$ is applied to the perceived rate of strain tensor, $\mathbf{S}(t)$. Only then a transformation into the eigenframe of $\mathbf{S}_{0}$ is performed. I would like to stress that the width and length of the filter kernel were chosen solely by intuition since a clear decision aid as used earlier (see figure 2.6) was not found. As a consequence, the precision of $\mathbf{S}_{1}$ is uncertain.

\subsection{Experimental Results}

Figure 4.2 shows the size evolution of tetrahedra in comparison to the mean squared separation of particle pairs for the same flow and the same initial separation (resp. edge length). It can be seen that the results for tetrahedra and pairs agree within 5\%, confirming that the size evolution of tetrahedra and the separation evolution of pairs provide the same information. One also finds that the fluctuations of the tetrahedra datasets are a bit larger due to the strong reduction in statistics, with approximately 20 -times less realizations than seen for pairs. The tetrahedra statistics therefore do not reach the same accuracy as the pair statistics, especially at later times. As a consequence, the very small $t^{3}$ effect is hardly detectable. As shown in section 4.1, however, the shape evolution of tetrahedra exhibits symmetry-breaking already at first order in $t$. This is a much larger effect, and it can be well analyzed with the obtained tetrahedra data and will be the focus of the following analysis. 


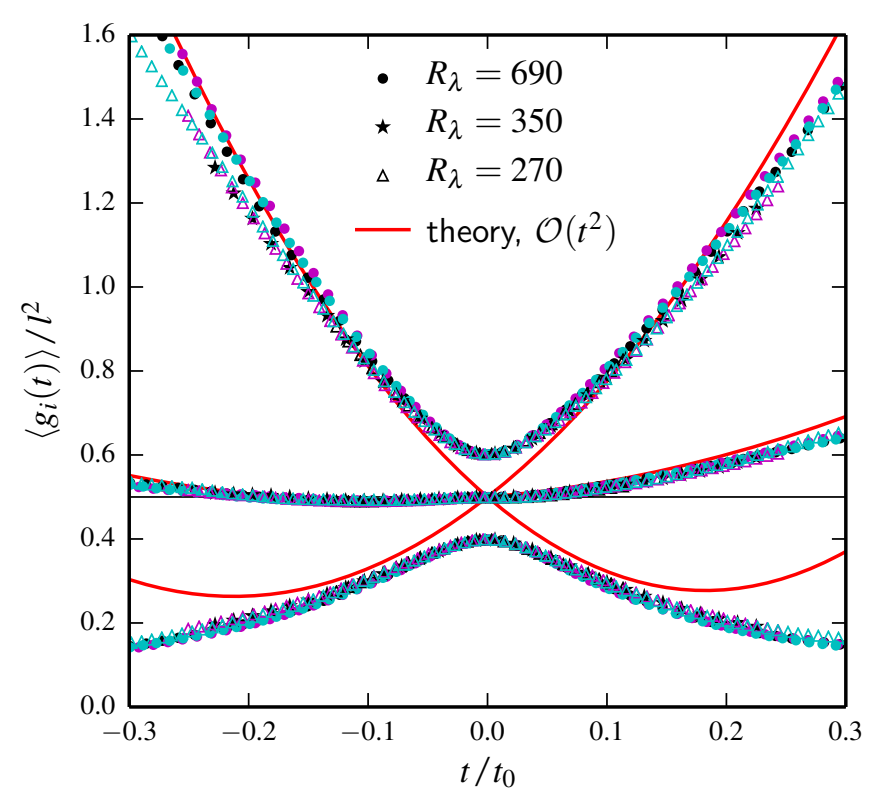

Figure 4.3.: Experimental results for the eigenvalues of the shape tensor, $\mathbf{G}(t)$, obtained from initially nearly regular tetrahedra with edge length $l$. The eigenvalues are nondimensionalized with $l^{2}$, and the variation range of the edge length, $\Delta l / l$, is as large as $10 \%$. For $R_{\lambda}=690$, the initial edge lengths are $l=364 \eta(\bullet), 424 \eta(\bullet)$, and $485 \eta(\bullet)$. For $R_{\lambda}=350, l=273 \eta(\star)$ and for $R_{\lambda}=270, l=135 \eta(\triangle), 154 \eta(\triangle)$, and $173 \eta$ $(\triangle)$ are shown. A theoretical prediction up to order $t^{2}$ as obtained from equation (4.18) is shown for comparison (-). For this prediction, measured strain quantities from the small measurement volume dataset at $R_{\lambda}=350$ and $l=182 \eta$ were used. More details can be found in the text.

Figure 4.3 shows the experimental results for the evolution of the eigenvalues $g_{i}(t)$ as a function of time, for both positive and negative times, together with the theoretical prediction up to order $t^{2}$. For the theoretical prediction, the measured strain quantities $\left\langle s_{i}\right\rangle,\left\langle S_{1, i i}\right\rangle$, and $\left\langle s_{i}^{2}\right\rangle$ from the small measurement volume dataset with $R_{\lambda}=350$ and $l=182 \eta$ have been inserted into equation (4.18). Only the first two orders in time could be used since the parameters needed for the $t^{3}$-term could not be fully resolved by the available data. Strain parameters from different initial separations and Reynolds numbers differ little and yield the same theoretical predictions. A complete list of the obtained strain quantities is given in appendix B.2.

It can be seen that, despite the large variation of the presented datasets concerning Reynolds number and initial edge length, all datasets collapse very well. The theoretical prediction agrees with the experimental results for $0.08 t_{0} \lesssim|t| \lesssim 0.2 t_{0}$. Discrepancies are observed for very short and very large times. The latter can be attributed to the negligence of the 


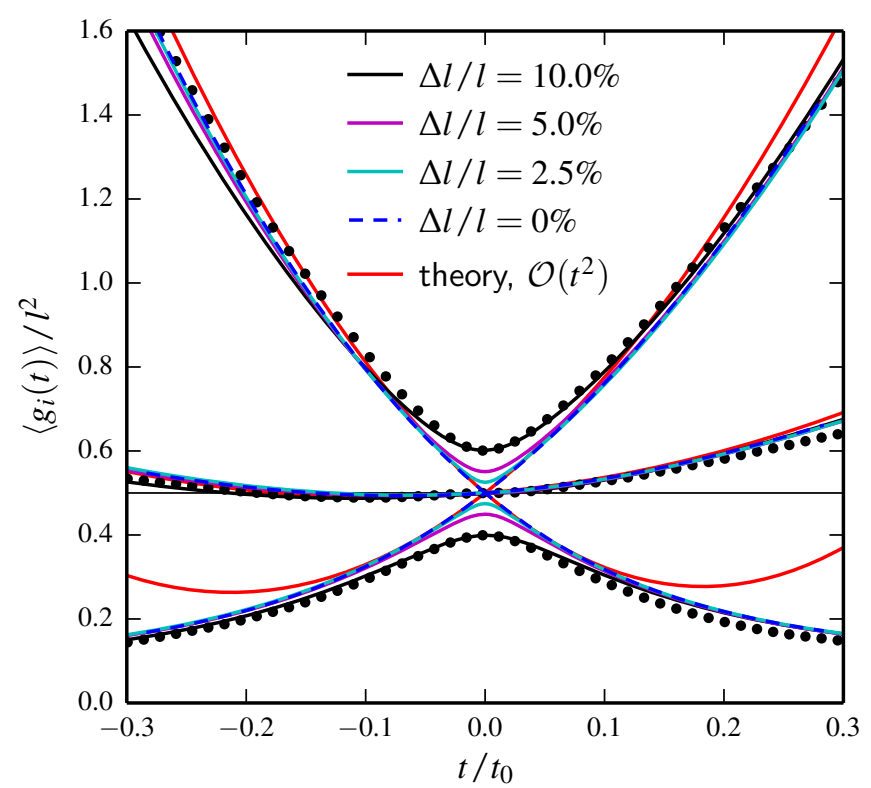

Figure 4.4.: Effect of the variations of the edge length, $\Delta l / l$, on the evolution of the eigenvalues of the shape tensor $\mathbf{G}(t)$. Lines show numerical results for the eigenvalues of the shape tensor obtained from initially (nearly) regular tetrahedra with edge length $l$. The variations of the edge length, $\Delta l / l$, are varied from $0 \%$ to $10 \%$. For $\Delta l / l=0 \%$, the Reynolds number is $R_{\lambda}=430$ and the initial edge length is $l=167 \eta$. For the other curves, $R_{\lambda}=300$ and $l=123 \eta$. An experimental dataset at $R_{\lambda}=690$ with $l=364 \eta(\bullet)$ and the theoretical prediction up to order $t^{2}(-)$ are shown for comparison.

$t^{3}$-term as well as the unknown and possibly large uncertainty of the first time derivative of the strain tensor, $\mathbf{S}_{1}$. The discrepancy at very short times is due to a deviation of the experimental initial conditions from the regular shape assumed in the theoretical prediction in equation (4.18). For an initially regular tetrahedron, all three eigenvalues should have a value of $\frac{l^{2}}{2}$ at $t=0$, as shown by the theory curve in figure 4.3. This is not the case for the shown experimental data, however, because the tetrahedra found experimentally are not perfectly regular. As stated earlier, in order to obtain sufficient statistical convergence, the lengths of the edges of a tetrahedron only need to fall into the range $[0.9 l, 1.1 l]$ for the tetrahedron to be counted as regular. Therefore, the three shape eigenvalues can deviate by a fair amount from the prescribed value. However, the initial eigenvectors of the shape tensor are randomly distributed and have no reason to be correlated with the eigenvectors of the perceived rate of strain tensor. Therefore, the tetrahedra deformation at $t=0$ only influences the evolution of the $g_{i}(t)$ for a very short time after which the theoretical behavior described in equation (4.18) is recovered. This was carefully confirmed by using DNS data 


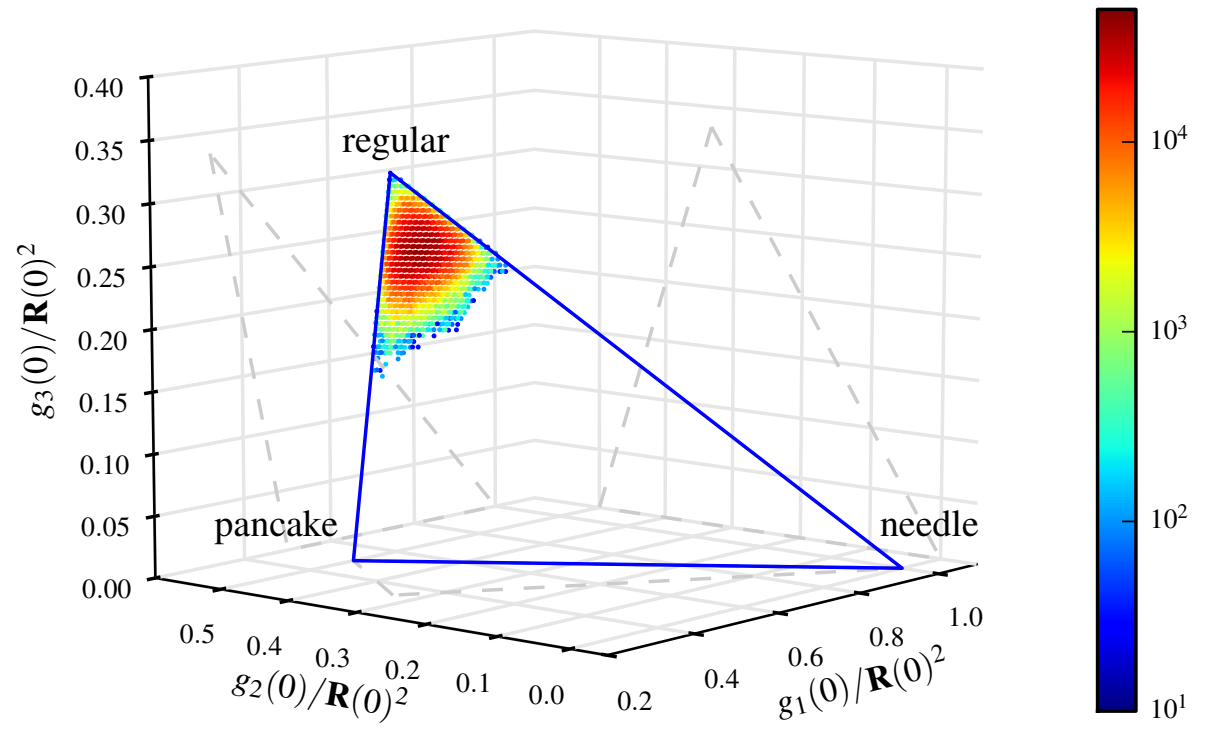

Figure 4.5.: Probability distribution of the measured shape eigenvalues $g_{i}(t)$ at $t=0$, normalized by the radius of gyration. The computed histogram covers the whole shown volume with a bin size of $(0.005)^{3}$. Only points with non-vanishing probabilities are depicted. The shown dataset was recorded at $R_{\lambda}=690$ and with $l=364 \eta$. The variation of the edge length, $\Delta l / l$, is $10 \%$.

at $R_{\lambda}=430^{1}$ and $R_{\lambda}=300^{2}$. Figure 4.4 shows the evolution of initially (nearly) regular tetrahedra, with the acceptance range for the initial edge lengths varied from $0 \%$ (perfectly regular) to $10 \%$ (experimental conditions). For comparison, an experimental dataset and the theoretical prediction are shown in the same plot. One finds that the experimental data agrees very well with the DNS dataset with $\Delta l / l=10 \%$, while the DNS dataset with $\Delta l / l=0 \%$ corresponds to the expected theoretical behavior for perfectly regular tetrahedra. It can be seen that after $t / t_{0} \approx 0.08$, all experimental and DNS curves with different $\Delta l / l$ agree with each other.

It is educational to also look at the full p.d.f. of the eigenvalues $g_{i}(t)$ instead of only their mean value. Figure 4.5 shows the initial distribution of the three eigenvalues. Due to $\sum g_{i}(t)=\mathbf{R}^{2}(t)$, only two of the three eigenvalues are independent. For the normalized shape eigenvalues, $\frac{g_{i}(t)}{\mathbf{R}^{2}(t)}$, one therefore finds that all possible combinations have to be

\footnotetext{
${ }^{1}$ This dataset was obtained by using a numerical simulation of a turbulent flow field from the Johns Hopkins University database (Li et al. 2008, Yu et al. 2012), and seeding it with regular tetrahedra. Then, the evolution of the particle positions forwards and backwards in time was computed and the desired statistics were obtained. This was done by Alain Pumir from École Normale Supérieure de Lyon.

${ }^{2}$ This is the same DNS dataset as used in section 3.3.
} 


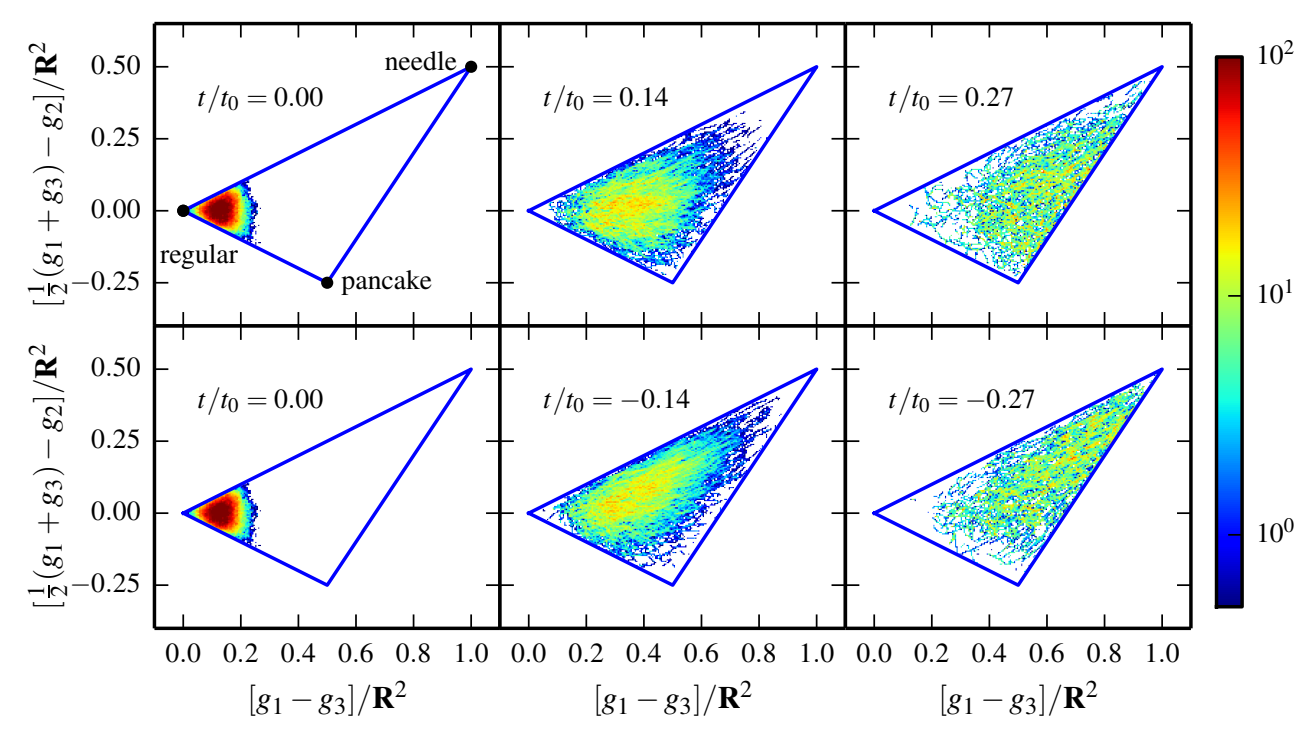

Figure 4.6.: Evolution of the probability distribution of the measured shape eigenvalues $g_{i}(t)$ at $t=0$, projected onto the triangular plane to which the shape configurations are confined. The special cases of regular $\left(g_{1}=g_{2}=g_{3}\right)$, pancake-shaped ( $g_{3}=$ $0)$ and needle-shaped $\left(g_{2}=g_{3}=0\right)$ tetrahedra can be found at the corners of the triangle. Evolution forwards and backwards in time is shown in the top and bottom row, respectively. The computed histogram has a bin size of $(0.005)^{2}$. Only points with non-vanishing probabilities are depicted. The shown dataset was recorded at $R_{\lambda}=690$ and with $l=364 \eta$. The variation of the edge length, $\Delta l / l$, is $10 \%$.

on the plane $\frac{g_{1}(t)}{\mathbf{R}^{2}(t)}+\frac{g_{2}(t)}{\mathbf{R}^{2}(t)}+\frac{g_{3}(t)}{\mathbf{R}^{2}(t)}=1$, where $0 \leq \frac{g_{i}(t)}{\mathbf{R}^{2}(t)} \leq 1$. Due to the sorting of the eigenvalues, $g_{1}(t) \geq g_{2}(t) \geq g_{3}(t)$, the plane of possible combinations is further reduced to a triangular surface, depicted in blue in figure 4.5. While a regular tetrahedron has $\frac{g_{1}(t)}{\mathbf{R}^{2}(t)}=\frac{g_{2}(t)}{\mathbf{R}^{2}(t)}=\frac{g_{3}(t)}{\mathbf{R}^{2}(t)}=\frac{1}{3}$ (the upper corner of the triangle), one can see that the distribution of the initial shape of the tetrahedra peaks a little away from this symmetry point.

In order to see the time evolution of the shape p.d.f. more clearly, it is useful to only display the plane onto which the shape configurations are confined. This is done in figure 4.6, where the new variables, $\frac{g_{1}(t)-g_{3}(t)}{\mathbf{R}(0)^{2}}$ and $\frac{0.5\left(g_{1}(t)+g_{3}(t)\right)-g_{2}(t)}{\mathbf{R}(0)^{2}}$, are two orthogonal vectors spanning the plane. It can be seen that both forwards and backwards in time, the shape of the tetrahedra become nearly coplanar with the distinction that for $t<0$ the values of $\left(g_{1}-g_{3}\right) / \mathbf{R}^{2}$ are larger. This means that for negative times, the coplanar shape is slightly more needle-like than for $t>0$. Similar findings were also reported by other studies (Pumir et al. 2000, Biferale et al. 2005, Xu et al. 2008, Hackl et al. 2011), but with a different choice of projection plane.

This observed time asymmetry can also be seen from the mean eigenvalues of the shape tensor, as shown in figure 4.3 and figure 4.4. As discussed earlier, the major influence on the short time evolution of the eigenvalues $g_{i}(t)$ stems from the eigenvalues of the 
perceived rate of strain tensor at $t=0, s_{i}(0)$. More explicitly, at first order in $t$, one has $\left\langle g_{i}(t)\right\rangle=\frac{l^{2}}{2}\left(1+2\left\langle s_{i}\right\rangle t+\mathcal{O}\left(t^{2}\right)\right)$. It is furthermore crucial to note that $\left\langle s_{2}\right\rangle>0$. Due to the sorting of the shape eigenvalues by size, it is clear that $\left\langle g_{1}(t)\right\rangle$ grows with $\left\langle s_{1}\right\rangle$ for $t>0$, while it grows with $\left\langle-s_{3}\right\rangle$ for $t<0$. The total asymmetry amounts to the difference between the two, with

$$
\frac{\left\langle g_{1}(t)-g_{1}(-t)\right\rangle}{l^{2}\left(t / t_{0}\right)}=\left\langle s_{1}+s_{3}\right\rangle t_{0}+\mathcal{O}(t)=-\left\langle s_{2}\right\rangle t_{0}+\mathcal{O}(t)
$$

where I used that $\left\langle s_{1}+s_{2}+s_{3}\right\rangle=0$. Note that the eigenvalues of the strain tensor have been non-dimensionalized with $t_{0}$ in order to make them independent of Reynolds number and initial edge length. An analogous expression can be found for the third eigenvalue, $\left\langle g_{3}(t)\right\rangle$. It is important to note that the asymmetry found in equation (4.19) is a direct consequence of the non-zero intermediate eigenvalue of the perceived rate of strain tensor. It does not occur in artificial flows with Gaussian or otherwise time-symmetric flow profiles, but reflects an intrinsic property of turbulent flows.

An even larger time asymmetry can be found for the intermediate eigenvalue of the shape tensor, for which

$$
\frac{\left\langle g_{2}(t)-g_{2}(-t)\right\rangle}{l^{2}\left(t / t_{0}\right)}=\left\langle s_{2}+s_{2}\right\rangle t_{0}+\mathcal{O}(t)=2\left\langle s_{2}\right\rangle t_{0}+\mathcal{O}(t) .
$$

Figure 4.7 shows relation (4.20) as a function of time for all analyzed experimental and numerical datasets. The solid horizontal line represents the value $2\left\langle s_{2}\right\rangle t_{0}=0.42$ as obtained from figure 4.1. It can be seen that while for very short times, $t<0.05 t_{0}$, all curves with a non vanishing variation of the edge length, $\frac{\Delta l}{l} \neq 0$, show a steeply rising slope, a plateau is observed for intermediate times with a value close to the prediction. The wide extension of the plateau might be explained by the argument that by taking the difference $\left\langle g_{2}(t)-g_{2}(-t)\right\rangle$, all higher order terms with an even power in $t$, especially the $t^{2}$-term, cancel out ${ }^{3}$. Therefore, the dominating, non-vanishing $t$-term is followed by a much weaker $t^{3}$-term. Similar to the two-particle case, it can be shown that all odd terms have an intrinsic timescale of $t_{0}$, so that the $t^{3}$-term only becomes important at $t \approx t+0$. The fact that all experimental curves stay slightly below the predicted plateau height might be attributed to the finite volume effect. Since the trajectories are not symmetrized around $t=0$ and fast separating tetrahedra are tracked for shorter periods than slowly separating tetrahedra, some of the asymmetry is lost at later times. In figure 3.4, I showed that the impact of the bias becomes of the order of $10 \%$ at $t / t_{0} \approx 0.1$. Therefore, also taking into account the measurement uncertainty of $\varepsilon$, a plateau at values 10 to $20 \%$ below the theoretical prediction is reasonable. In total, figure 4.7 confirms the theoretical prediction that the asymmetry of the evolution of the intermediate eigenvalue of the shape tensor to leading order is given by $2\left\langle s_{2}\right\rangle$, even for tetrahedra which

\footnotetext{
${ }^{3}$ In contrast to the two-particle case in chapter 3, the observation length of the tetrahedra trajectories forwards and backwards in time were not symmetrized. Therefore, the bias effect due to the finite measurement volume is not fully time-symmetric and cannot be expected to cancel out exactly when taking the difference $\left\langle g_{2}(t)-g_{2}(-t)\right\rangle$. Since the focus is on the non-vanishing first-order term, however, the finite volume bias is assumed to have no qualitative effect on the presented tetrahedra data.
} 


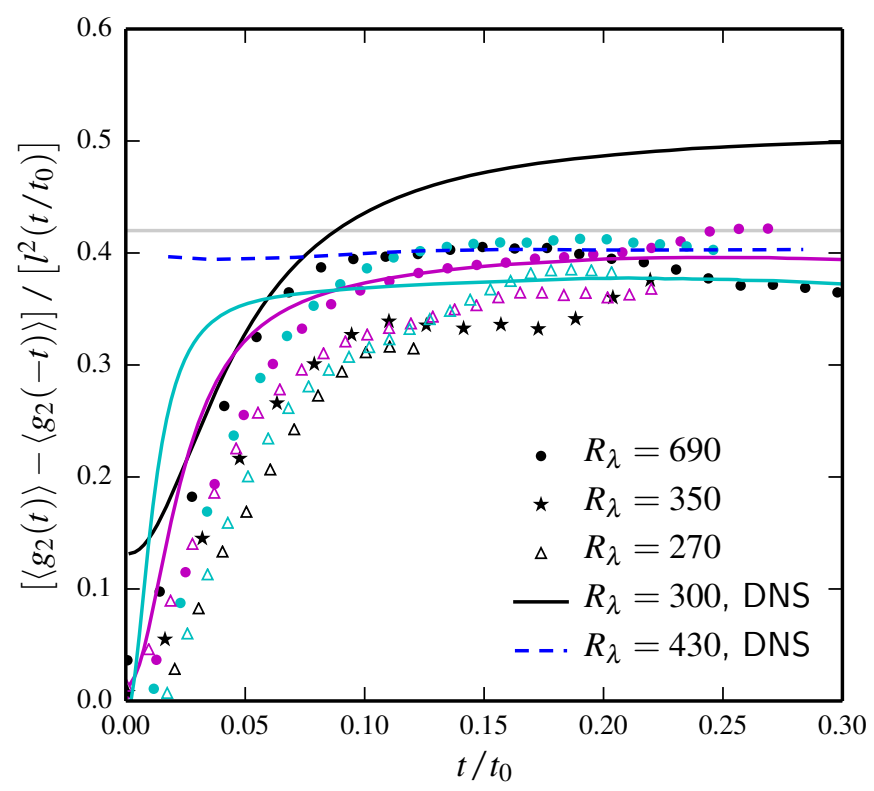

Figure 4.7.: Asymmetry of the intermediate shape eigenvalue, compensated by $t / t_{0}$. For experimental data at $R_{\lambda}=690$, the initial edge lengths are $l=364 \eta(\bullet), 424 \eta(\bullet)$, and $485 \eta(\bullet)$, for $R_{\lambda}=350, l=273 \eta(\star)$ and for $R_{\lambda}=270, l=135 \eta(\triangle), 154 \eta$ $(\triangle)$, and $173 \eta(\triangle)$. Solid lines represent DNS data at $R_{\lambda}=300$ with $l=123 \eta$. The variations of the edge length, $\Delta l / l$, are $10 \%(-), 5 \%$ (一) and 2.5\% (一). The dashed line represents DNS data at $R_{\lambda}=430$ with an initial edge length of exactly $l=167 \eta$ $(\Delta l / l=0 \%,--)$. The horizontal gray line depicts the value of $2\left\langle s_{2}\right\rangle t_{0}=0.42$ from figure 4.1.

are only approximately regular at $t=0$.

So far, I studied the time asymmetry in pair dispersion and in the shape evolution of tetrahedra. I found that in both cases, the observed asymmetry is directly linked to intrinsic properties of turbulent flows. For pair dispersion, the symmetry-breaking $t^{3}$ term was found to be directly proportional to the energy transfer rate of the turbulence energy cascade. For tetrahedra, I found that symmetry breaking already occurs at leading order in $t$ due to a non-vanishing intermediate eigenvalue, $s_{2}$, of the perceived rate of strain tensor. As discussed by Betchov (1956) for the true strain tensor in the dissipative range, the non-zero value of $s_{2}$ arises directly from a positive, non-zero viscous term. It seems plausible that this relation carries over to some extent into the inertial range, therefore linking the asymmetry in tetrahedra deformation to the energy cascade. As a next step, it thus seems prudent to change the energy cascade and analyze how this affects the observed time asymmetry. This change of the energy cascade will be realized by adding minute amounts of polymers to the flow, as will be discussed in detail in the next chapter. 



\section{CHAPTER 5 \\ The Effect of Polymers}

It is known for more than sixty years (Toms 1948) that the addition of minute amounts of polymers to a turbulent flow, even as low as some parts per million, strongly reduces the drag as shown in figure 5.1. Partly owing to the great usefulness of this finding, especially for the reduction in power consumption when pumping fluids through pipes, a lot of research has been focused in this direction (a comprehensive overview can e.g. be found in Lumley 1969, Hoyt 1972, Virk 1975, White \& Mungal 2008, Procaccia et al. 2008). The underlying fundamental processes of this effect are, however, still unclear.

Long-chain polymers usually possess a high degree of flexibility and, in their basic state, coil up to an approximately spherical shape. In a turbulent flow, the polymers become stretched and some of the kinetic energy of the fluid is stored in the elastic degrees of freedom of the polymer. Since the stretched polymers are still much smaller than the Kolmogorov length scale and only minute amounts are added, it is a great puzzle how they can so profoundly alter the properties of the flow.

In this chapter, I study the effect of polymers on a turbulent flow as observed from the dispersion of clusters of two and four particles. In section 5.1, I briefly describe how the polymer solution was produced and filled into the tank. In section 5.2, I present some important theories on polymer-flow interactions and show experimental results for the explicit flow considered in this thesis. In sections 5.3 and 5.4, I finally show how polymer-flow interactions can be analyzed with the methods introduced in the previous two chapters.

\subsection{Preparation of the Experiment}

In order to study the effect of polymers on the flow, the cylindrical tank of the experimental setup is first filled with deionized water and then a high-concentration polymer solution is added so that the resulting mixture had the desired polymer concentration. For the concentrated polymer solution, small amounts of polyacrylamide with a molecular weight 


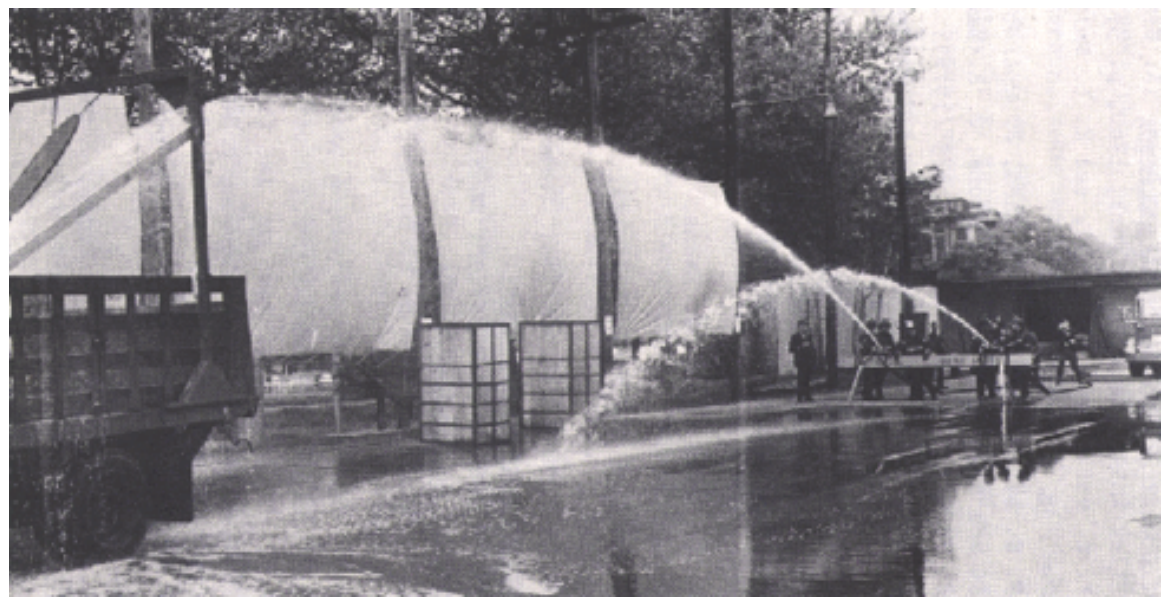

Figure 5.1.: This picture shows two water jets from identical fire hoses. Both hoses were aligned at the same angle and, for pure water, produced jets of equal reach. Then, a diluted solution with $30 \mathrm{ppm}$ of high molecular weight poly(ethylene oxide) was used for the left hose. As a result, the jet exited at a higher speed, was more coherent, and had a significantly enhanced reach. (Reprinted from Bailey \& Koleske 1976, chap. 4, p. 81.)

of $18 \cdot 10^{6} \mathrm{u}$ (Polysciences 18522) are mixed into a specified amount of deionized water ( $1-2$ liters) with a stirrer. For the first minute, the rotation rate of the stirrer is set to $450 \mathrm{rpm}$, then it is decreased to $300 \mathrm{rpm}$ and the solution is stirred for another approximately $30 \mathrm{~min}$. This way, a good dispersion of the polymers is achieved without exerting too much force on the polymer chains for them to break. This is important since the length of the polymers is a determining factor for the influence of the polymers on the flow properties. Then, the polymer solution is filled into bottles and rotated slowly over night (figure 5.2(a)). Due to the long and slow mixing, the polymers disperse very well in the solution, building only very few clumps of entangled polymers. It is expected that these clumps dissolve at the latest when the concentrated polymer solution is mixed into the $120 \mathrm{l}$ of the tank content and the clumps are exposed to the turbulent flow.

The polymer solution is filled into the tank through the small bucket on the top-plate (figure 5.2(b)). The little pump connected to the bucket is not used to pump the polymer solution into the tank since the polymer chains could be damaged. Instead, some of the deionized water in the tank is drained through a valve in the bottom plate so that the polymer solution in the bucket is sucked in. When filling the polymer solution into the bucket, some rest amount of deionized water, $V_{b}=400 \pm 10 \mathrm{ml}$, needs to remain in the bucket to avoid that air is sucked into the tank. It is assumed, that when filling in the polymer solution, the rest amount of water is always drained first. Since this is not certain, the remaining water in the bucket poses a large source of uncertainty in the determination of the polymer concentration in the tank. In order to reduce this error, the amount of the remaining water in the bucket is reduced to $V_{b}=200 \pm 10 \mathrm{ml}$ by inserting a large, heavy weight into the 


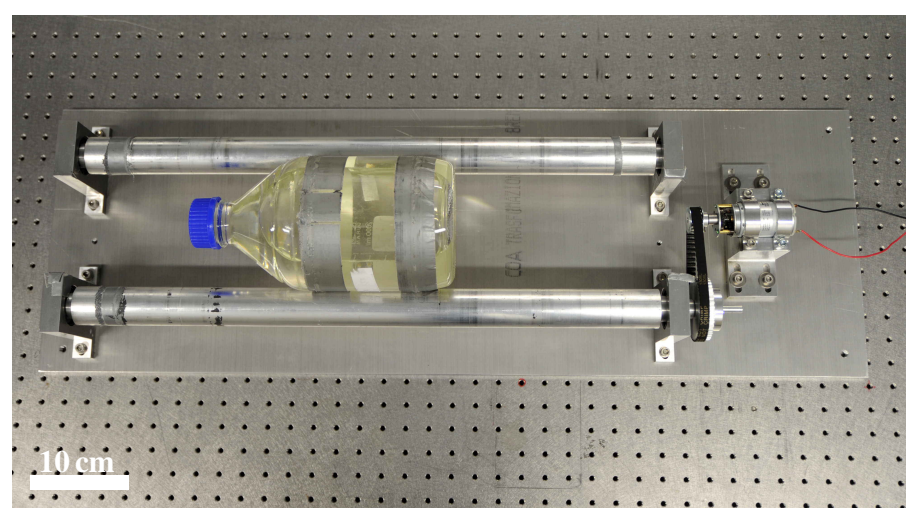

(a) Rotation Device

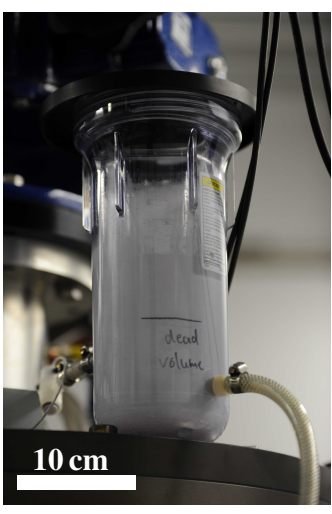

(b) Bucket

Figure 5.2.: (a) Picture of the self-built rotation device, used for gently mixing highly concentrated polymer solution. (b) Picture of the small bucket mounted on top of the tank. The bucket can be used for introducing substances into the tank via small tubes connected to the top and bottom plate of the tank. If the water level in the bucket drops below a certain level, marked "dead volume", air bubbles may enter the tank.

bucket, thus displacing some of the water ${ }^{1}$. Furthermore, after draining the first full bucket of solution into the tank, the bucket is filled with the remaining solution which is stirred carefully into the remainder of the first filling. This way, the worst possible case, namely that no residual water is drained, is replaced by the case that only a small part of the water, corresponding to a well mixed solution, is not drained.

The amount of polyacrylamide in the tank, $m_{\mathrm{in}}$, is finally given by

$$
m_{\mathrm{in}}=m_{\mathrm{pol}}\left(1-\frac{V_{w}}{V}\right)
$$

with $m_{\text {pol }}$ the amount of polymers in the concentrated polymer solution, $V$ the amount of water used for the concentrated polymer solution and $V_{w}$ the amount of the remaining water in the bucket that is also drained into the tank. In the expected case that all the residual water is drained, one has $V_{w}=V_{b}$. The value of $V_{w}$ can however vary between $V_{w}=V_{b}+10 \mathrm{ml}$, taking into account the measurement uncertainty of $V_{b}$, and $V_{w}=\frac{V_{b} V}{V+V_{b}}$, corresponding to the worst case in which the solution is well mixed, and a small fraction of the water is not drained $^{2}$. The error bounds on $V_{w}$ are thus given by $\Delta V_{w}={ }_{-V_{b}^{2} /\left(V+V_{b}\right)}^{+10 \mathrm{ml}}$. With an accuracy for the weight measurement of $\Delta m_{\mathrm{pol}}=0.005 \mathrm{~g}$ and for the volume of the mixing water of $\Delta V=50 \mathrm{ml}$, the measurement error on the amount of polymers inserted into the tank is given by

$$
\Delta m_{i n}=\left|\frac{V-V_{w}}{V} \Delta m_{\mathrm{pol}}\right|+\left|m_{\mathrm{pol}} \frac{V_{w} \Delta V}{V^{2}}\right|+\left|m_{\mathrm{pol}} \frac{\Delta V_{w}}{V}\right| .
$$

\footnotetext{
${ }^{1}$ This was only done after some time. For the first two datasets, it was $V_{b}=400 \mathrm{ml}$

${ }^{2}$ Here, the small uncertainty of $V_{b}$ is neglected.
} 
The error on the concentration is then simply given by

$$
\Delta c / c=\left|\Delta m_{\text {in }} /\left(V_{\text {tank }} \rho_{w}\right)\right|+\left|m_{\text {in }} /\left(V_{\text {tank }}^{2} \rho_{w}\right) \Delta V_{\text {tank }}\right|,
$$

with $V_{\text {tank }}=120 \pm 21$ the full volume of the tank and $\rho_{w}$ the density of water (assumed to be exact). The resulting concentration errors for the individual datasets are given in table 2.2.

Aside from the added polymers, the measurement procedure is exactly as described in chapter 2.

\subsection{Effect of Polymers on the Energy Cascade}

Flexible polymer chains in a turbulent flow are stretched by straining regions of the flow (Tabor \& De Gennes 1986, De Gennes 1986, Smith et al. 1999), thus removing some of the energy from the flow field and storing it in the elastic degrees of freedom of the polymers (see figure 5.3). This energy is then released at the size scale of the polymers, which is much smaller than the Kolmogorov scale $\eta$. With this known, the question arises of how objects of such a small size can have such a profound influence on the flow properties as observed in experimental studies. This is even more puzzling taking into consideration the very small amounts of polymers needed to obtain an effect (only a few parts per million).

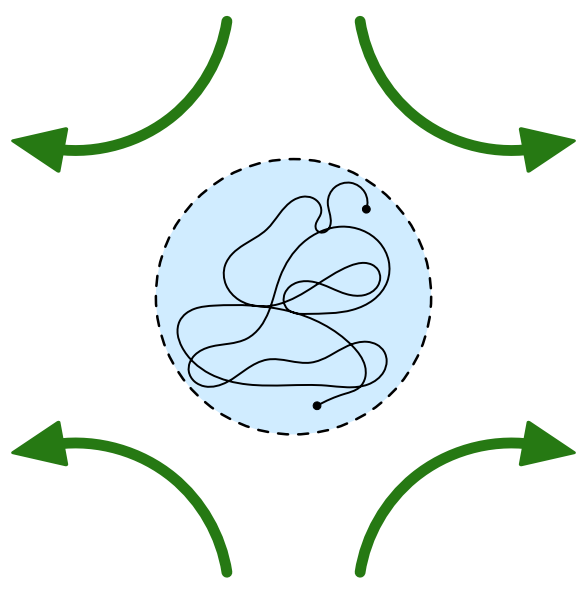

(a) Coiled state

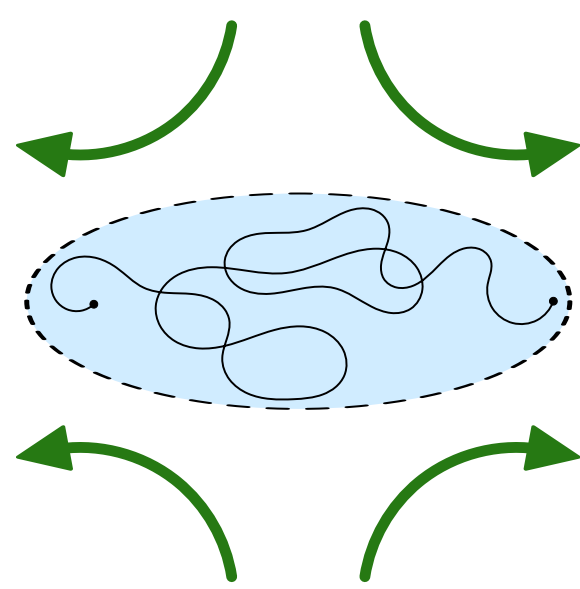

(b) Stretched state

Figure 5.3.: Sketch of the transition from a coiled to a stretched state of a single polymer molecule due to a straining flow pattern.

The first to propose a well accepted explanation to this riddle was Lumley (1969) who reasoned that one has to look at the time scales involved, not at the length scales. He argued that the polymers can be stretched by all eddies with time scales $\tau_{r}$ faster than the relaxation time $\tau_{p}$ of the polymer, where $\tau_{p}$ is the time that a stretched polymer chain needs to relax back into its energetically preferable coil configuration (Zimm 1956). For a linear polymer 
chain with $N$ monomers of length $a$, the relaxation time can be given by $\tau_{p} \approx \frac{\mu}{k T}\left(N^{\frac{3}{5}} a\right)^{3}$, with $k=1.38 \cdot 10^{-23} \mathrm{~J} / \mathrm{K}$ being the Boltzmann constant, and $\mu$ and $T$ the dynamic viscosity and temperature of the solution. From Lumley's time-criterion, one then obtains a maximum eddy size $r^{*} \propto\left(\varepsilon \tau_{p}^{3}\right)^{\frac{1}{2}}$ below which polymer-flow interactions could occur. This length scale is much larger than the Kolmogorov scale, thus providing some explanation for the large effect of polymers on the flow. Lumley, however, did not incorporate the effect of different polymer concentrations.

About a decade later, de Gennes and Tabor constructed a more complete model (De Gennes 1986, Tabor \& De Gennes 1986). They suggested that for $r<r^{*}$, which are those scales involved in polymer stretching according to Lumley, the polymer elastic energy per volume is given by $E_{e}(r) \propto c_{p} k T\left(r^{*} / r\right)^{\frac{5 n}{2}}$ with $c_{p}$ being the number of polymer chains per unit volume and $n$ being an unknown constant ${ }^{3}$. They then argued that below a scale $r^{* *}$, at which the polymer elastic energy equals the turbulence kinetic energy,

$$
E_{e}\left(r^{* *}\right)=E_{k}\left(r^{* *}\right)=\rho \mathbf{u}^{2}\left(r^{* *}\right),
$$

the turbulence energy cascade will be truncated since most of the energy is absorbed by the polymers. This scale is obtained from equation (5.4) to be

$$
r^{* *} \propto\left(\frac{k T c_{p}}{\rho} \varepsilon^{(5 n / 4-2 / 3)} \tau_{p}^{15 n / 4}\right)^{1 /(2 / 3+5 n / 2)} .
$$

Note that the resulting scale $r^{* *}$ is naturally always smaller than the Lumley scale $r^{*}$.

According to this theory, the turbulent energy cascade is thus assumed to be unchanged at scales larger than $r^{* *}$. This, however, contradicts the initial assumption of the theory that polymers gain elastic energy from all eddies with sizes $r<r^{*}$, ergo including eddies in the range $r^{* *}<r<r^{*}$, and thereby already remove some kinetic energy from the flow at scales larger than $r^{* *}$. Xi et al. (2013) concluded from this that it is not the energy balance itself one should look at but the balance of energy flux. Assuming that the characteristic polymer time scale to dissipate energy is $\tau_{p}$, they defined the elastic energy flux as

$$
\varepsilon_{p}(r) \propto \frac{E_{e}(r)}{\rho \tau_{p}} \propto \frac{k T c_{p}}{\rho \tau_{p}}\left(\frac{r^{*}}{r}\right)^{5 n / 2} .
$$

One can then derive a new length scale $r_{\varepsilon}$ at which the turbulent energy flux, $\varepsilon$, equals the energy flux due to the polymers, $\varepsilon_{p}(r)$. This scale is given by

$$
r_{\varepsilon}=A\left(\frac{k T}{\rho}\right)^{2 /(5 n)} c_{p}^{2 /(5 n)} \varepsilon^{1 / 2-2 /(5 n)} \tau_{p}^{3 / 2-2 /(5 n)},
$$

where $A$ is an unknown proportionality constant that depends on the polymer type. They showed that experimentally measured second order structure functions at different polymer

\footnotetext{
${ }^{3}$ The expression for the polymer elastic energy is derived based on polymers in elongational laminar flow fields under the assumption that the stretching behavior of the polymers is similar in a turbulent flow (De Gennes 1986).
} 
concentrations and Reynolds numbers can be made collapse on one curve when the spatial scale is normalized with $r_{\varepsilon}$, indicating that this is indeed the right length scale for the problem. Furthermore, for the constants $n$ and $A$, they obtain $n=1.0 \pm 0.2$ and $A=101 \pm 17$ (for polyacrylamide) (Xi et al. 2013).

In this thesis, I do not use the second order structure function but $\langle\delta \mathbf{u} \cdot \delta \mathbf{a}\rangle=-2 \varepsilon$ as a measure for the energy flux. Nevertheless, the above arguments should still hold. Figure 5.4 shows the curves for $-\frac{1}{2}\langle\boldsymbol{\delta} \mathbf{u} \cdot \boldsymbol{\delta} \mathbf{a}\rangle$ as functions of $r$ for datasets with different polymer concentrations. All shown datasets were recorded at a propeller frequency of $0.9 \mathrm{~Hz}$, corresponding to a Reynolds number of $R_{\lambda}=350$ for the water case. One observes that $-\frac{1}{2}\langle\delta \mathbf{u} \cdot \delta \mathbf{a}\rangle$ decreases at all resolvable scales with increasing polymer concentration. In principle, the difference between the curves with and without polymers is a measure for the elastic energy flux $\varepsilon_{p}(r)$. However, the polymers can also interfere with the energy injection into the flow due to boundary effects, and they have been shown to reduce large-scale velocity fluctuations (Ouellette et al. 2009, Xi et al. 2013). Since these boundary and large-scale effects are not the focus of this study, the reduction of the overall turbulence energy flux is taken into account by defining a different value of $\varepsilon$ for each curve, using a horizontal fit to the plateau in the inertial range ${ }^{4}$.

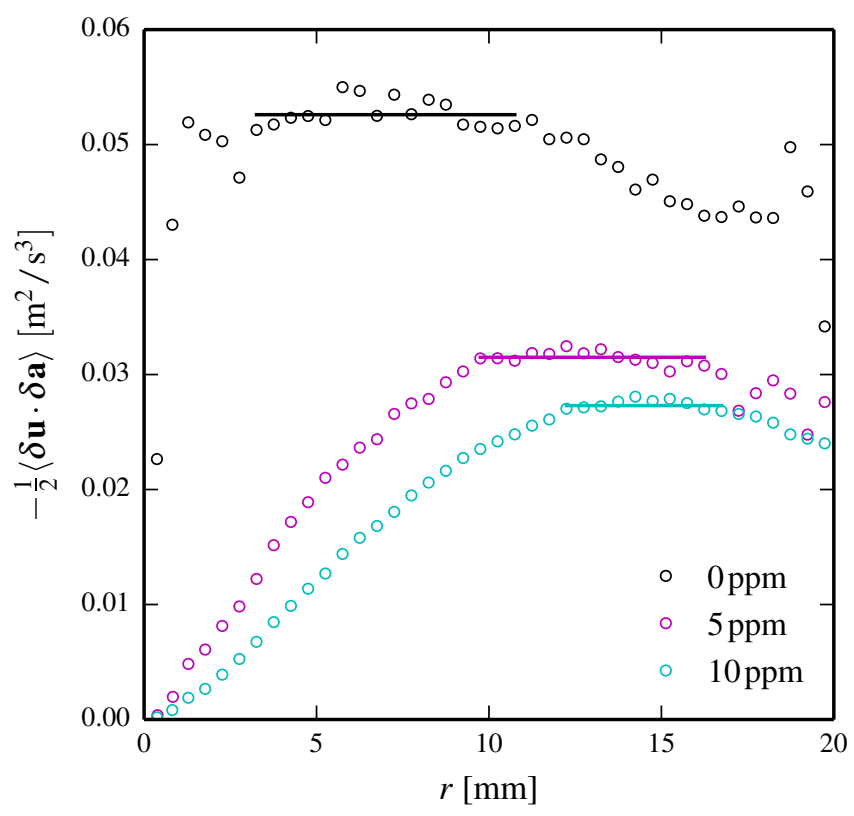

Figure 5.4.: Measured values for $-\frac{1}{2}\langle\delta \mathbf{u} \cdot \delta \mathbf{a}\rangle$ as a function of scale for three different polymer concentrations. All three data sets are recorded with the same propeller frequency of $0.9 \mathrm{~Hz}$, corresponding to the pure water dataset with $R_{\lambda}=350$ and $\eta=$ $0.058 \mathrm{~mm}$. Solid horizontal lines represent the fit to obtain $\varepsilon$.

\footnotetext{
${ }^{4}$ The resulting values for all polymer datasets are given in table 2.2 .
} 
Figure 5.5 (a) shows the same datasets rescaled by the heights of the plateaus. Furthermore, datasets at $R_{\lambda}=390$ were processed in the same way and are also shown here. The datasets at $R_{\lambda}=270$ will be omitted in this chapter because $\langle\delta \mathbf{u} \cdot \delta \mathbf{a}\rangle$ shows no sufficient plateau anymore if polymers are added (see appendix A.1 for the respective figures and a discussion). Now, the difference between the water-dataset and the different polymer-datasets indeed provides a measure for $\varepsilon_{p}(r)$. A shift of the onset of the plateau to higher scales with increasing polymer concentration is clearly visible here. In comparison, the effect due to the small difference in the Reynolds numbers is negligible. The general decrease of the plateau height and its shift to higher scales have also been seen for similar studies on the second order structure function (Ouellette et al. 2009, Xi et al. 2013). Further studies also observed a suppression of the small scales when adding polymers (Tong et al. 1992, Bonn et al. 1993, Liberzon et al. 2005, 2006, Berti et al. 2006, Crawford et al. 2008)
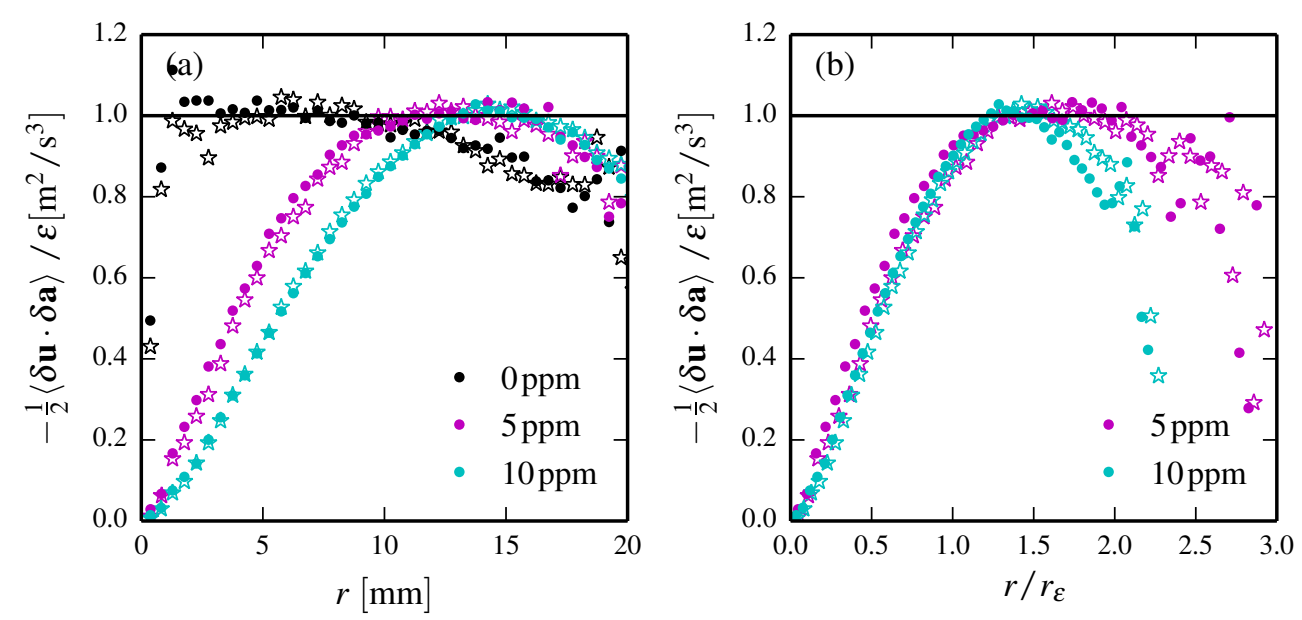

Figure 5.5.: (a) Measured values for $-\frac{1}{2}\langle\delta \mathbf{u} \cdot \delta \mathbf{a}\rangle$ rescaled with the respective value of $\varepsilon$ as a function of scale for three different polymer concentrations. Datasets recorded with a propeller frequency of $1.1 \mathrm{~Hz}$, corresponding to $R_{\lambda}=390$, are shown as circles $(\bullet, \bullet, \bullet)$. Datasets with a propeller frequency of $0.9 \mathrm{~Hz}$, corresponding to $R_{\lambda}=350$, are shown as stars ( 2 , 访, 访). (b) Here, the spatial scale is compensated by $r_{\varepsilon}$ and only datasets with added polymers are shown.

As a next step, I rescale $r$ with $r_{\varepsilon}$ as suggested by Xi et al. (2013). For the polyacrylamide used in this study, the radius of gyration of a polymer chain is given by $R_{g}=N^{\frac{3}{5}} a=0.5 \mu \mathrm{m}$. At $T=293 \mathrm{~K}$ and with a dynamic viscosity of of water of $\mu\left(20^{\circ} \mathrm{C}\right)=1.0 \cdot 10^{-3} \mathrm{Pas}$, this leads to a polymer relaxation time of $\tau_{p}=43 \mathrm{~ms}$. Using the respective $\varepsilon$ from the plateau fits and the measured values of $A=101$ and $n=1.0$ from Xi et al. (2013), one finds

$$
\begin{array}{lll}
R_{\lambda}=350: & r_{\varepsilon}(5 \mathrm{ppm})=7.6 \mathrm{~mm} \hat{=} 115 \eta, & r_{\varepsilon}(10 \mathrm{ppm})=10.0 \mathrm{~mm} \widehat{=} 152 \eta, \\
R_{\lambda}=390: & r_{\varepsilon}(5 \mathrm{ppm})=8.2 \mathrm{~mm} \widehat{=} 141 \eta, & r_{\varepsilon}(10 \mathrm{ppm})=10.7 \mathrm{~mm} \widehat{=} 184 \eta,
\end{array}
$$

where the values for $\eta$ where obtained from the water datasets with the respective Reynolds 
number. The results are shown in figure 5.5 (b). Please note that for the case without polymers, no $r_{\varepsilon}$ is defined so that these datasets are not shown. One finds a very good collapse for the different polymer concentrations, with the plateau being reached at $r \approx 1.2 r_{\varepsilon}$ for all datasets. These results support the theory by Xi et al. (2013) and confirm the values for $A$ and $n$.

It should be stated that the theory used here is based on the assumption that for all scales below the Lumley scale $r^{*}$, which is by construction much larger than the scale $r_{\varepsilon}$, some of the kinetic energy flux of the flow is diverted into the scale-dependent elastic energy flux of the polymers, $\varepsilon_{p}(r)$. Only above the Lumley scale, which in our case is assumed to lie at scales beyond the analyzed range, $\langle\delta \mathbf{u} \cdot \delta \mathbf{a}\rangle$ should yield the unchanged, constant energy transfer rate $\varepsilon$. As a consequence, the plateaus shown in figure 5.5 should not be perfectly flat but are indeed believed to exhibit a small increase with growing scales, meaning that a small $r / r_{\varepsilon}$ dependence of the datasets can remain, especially for scales at the lower boundary of the plateau. A second consequence is a possibly slightly too low value for $\varepsilon$ since the fit to $\langle\delta \mathbf{u} \cdot \delta \mathbf{a}\rangle$ is done in a region where $\langle\delta \mathbf{u} \cdot \delta \mathbf{a}\rangle$ did not yet reach its true plateau value. This difference is assumed to be negligible small in comparison to the overall uncertainty on $\varepsilon$ of $10 \%$, however.

So far, I found that adding small amounts of polymers has a noticeable effect on the energy transfer of the studied flow. This effect can be explained by a "redirection" of some of the kinetic energy flux of the flow to small scales by elastic stretching and relaxation of the polymers. In the next two sections, I want to analyze how this effect influences the dispersion of clusters of two and four particles.

\subsection{Two-Particle Dispersion}

As discussed in detail in chapter 3, the time asymmetry in pair dispersion can be studied by looking at the antisymmetric function

$$
\begin{aligned}
F_{t}^{a}(t) & =\frac{\left\langle\delta \mathbf{R}(t)^{2}-\delta \mathbf{R}(-t)^{2}\right\rangle_{t}}{2 R_{0}^{2}}=\frac{\langle\mathbf{V}(0) \cdot \mathbf{A}(0)\rangle_{0}}{R_{0}^{2}} f_{2}(t) t^{3}+\mathcal{O}\left(t^{5}\right) \\
& =-2 f_{2}(t)\left(\frac{t}{t_{0}}\right)^{3}+\mathcal{O}\left(t^{5}\right)
\end{aligned}
$$

with $t_{0}=\left(\frac{R_{0}^{2}}{\varepsilon}\right)^{\frac{1}{3}}$. Figure 5.6 shows the experimental results, where lines indicate the pure water case, and symbols indicate polymer concentrations of $5 \mathrm{ppm}$ (a) and $10 \mathrm{ppm}$ (b). As motivated in the previous section, different values for $\varepsilon$ for the polymer datasets were used. All curves are again compensated with $\frac{\langle\mathbf{V}(0) \cdot \mathbf{A}(0)\rangle_{0}}{R_{0}^{2}} t^{3}$, leading to a fixed plateau height of exactly one.

It can be seen that all curves for a certain polymer concentration nearly collapse on top of each other, with a possibly weak dependence on $r / r_{\varepsilon}$, while there is a clear difference between the cases with and without polymers. For water, most curves show a plateau up to $t \approx 0.05 t_{0}$, whereas the datasets with added polymers deviate already at $t \approx 0.005 t_{0}$, a factor 


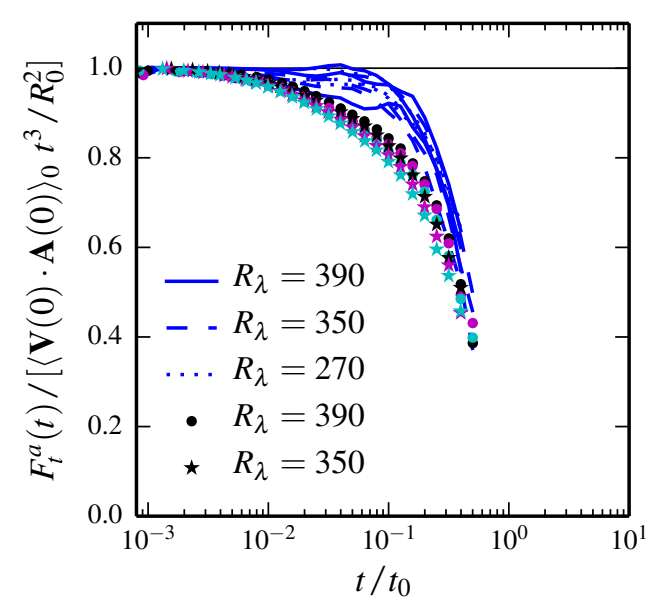

(a) $5 \mathrm{ppm}$

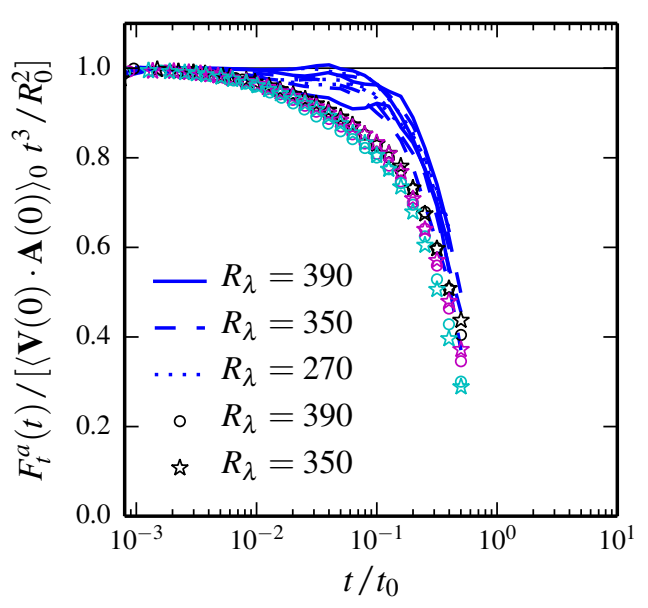

(b) $10 \mathrm{ppm}$

Figure 5.6.: Time-asymmetry in pair dispersion as seen from the compensated antisymmetric function $F_{t}^{a}(t)$ for two different polymer concentrations (symbols), $5 \mathrm{ppm}$ (a) and $10 \mathrm{ppm}$ (b), in comparison to the water case (lines). Datasets at three different propeller frequencies $\left(0.5 \mathrm{~Hz} \leftrightarrow R_{\lambda}=270,0.9 \mathrm{~Hz} \leftrightarrow R_{\lambda}=350,1.1 \mathrm{~Hz} \leftrightarrow R_{\lambda}=390\right)$ are shown. For the datasets with added polymers, different initial separations of $14 \mathrm{~mm}$, $16 \mathrm{~mm}$, and $18 \mathrm{~mm}$ are shown. In terms of the scale $r_{\varepsilon}$ defined in the previous section, the initial edge lengths for a concentration of $5 \mathrm{ppm}$ and $R_{\lambda}=390$ are $R_{0}=1.7 \mathrm{r}_{\varepsilon}(\bullet)$, $2.0 r_{\varepsilon}(\bullet)$, and $2.2 r_{\varepsilon}(\bullet)$ and for $R_{\lambda}=350$ they are $R_{0}=1.8 r_{\varepsilon}(\star), 2.1 r_{\varepsilon}(\star)$, and $2.4 r_{\varepsilon}(\star)$. For a concentration of $10 \mathrm{ppm}$, the initial edge lengths for $R_{\lambda}=390$ are $R_{0}=1.3 r_{\varepsilon}(\mathrm{O}), 1.5 r_{\varepsilon}(\mathrm{O})$, and $1.7 r_{\varepsilon}$ (○) and for $R_{\lambda}=350$ they are $R_{0}=1.4 r_{\varepsilon}$ (该), $1.6 r_{\varepsilon}$ (约), and $1.8 r_{\varepsilon}$ (放). For the water case, the same datasets as in figure 3.6 on page 55 are shown, but they are all colored blue for better contrast to the polymer datasets $(-,--, \cdots)$.

of 10 earlier. For the two different polymer concentrations, on the other hand, no significant difference can be observed.

These observations can have several reasons. One argument could be that the effect is caused artificially by using different $\varepsilon$-values for the water and polymer datasets, leading to different values of $t_{0}$ and thus of the deviation time. This argument can easily be refuted by noting that the difference between $\varepsilon_{0 \mathrm{ppm}}$ and $\varepsilon_{10 \mathrm{ppm}}$ for the same propeller frequency is at most a factor of two. Since $t_{0} \propto \varepsilon^{-\frac{1}{3}}$, this leads to $t_{0,10 \mathrm{ppm}} \approx 1.3 t_{0,0 \mathrm{ppm}}$, which is much too small to explain the observed effect ${ }^{5}$.

Assuming that $\varepsilon$ is chosen sufficiently precisely, the only other possibility is that the higher order terms, notably the $t^{5}$-term, are changed by the polymer-flow interaction. This change can be in terms of a modified prefactor or in the form of a new timescale. So far, I based my explanations of the convergence radius of the used Taylor-Expansion fully on the assumption

\footnotetext{
${ }^{5}$ The same reasoning of course also dismisses the measurement error on $\varepsilon$ as an explanation.
} 
that, in a turbulent flow, two-point functions do only involve the time-scale $t_{0}$ (Frishman 2013). With the addition of polymers, this assumption might not be valid anymore and the convergence of the Taylor expansion, especially of the odd terms, could be severely reduced. If the addition of polymers indeed introduces a new timescale, figure 5.6 indicates that this timescale, compensated by $t_{0}$, weakly depends on $r / r_{\varepsilon}$. The lacking difference between the two polymer concentrations also suggests that there is a critical concentration smaller than $5 \mathrm{ppm}$ beyond which any further addition of polymers does not lead to a change as long as the scales under consideration are chosen in the plateau range of $\langle\delta \mathbf{u} \cdot \delta \mathbf{a}\rangle$ and the corrected value for $\varepsilon$ is used. This roughly agrees with the findings of Ouellette et al. (2009) who see a critical concentration around $5 \mathrm{ppm}$, using the same polymer type. Within the uncertainties of the concentration measurements, it is reasonable to assume that the datasets presented here are both above the critical concentration observed by Ouellette et al. (2009).

Looking at the much richer statistics of four-particle clusters can help to gain further inside in this complex problem.

\subsection{Four-Particle Dispersion}

Since it was shown in section 4.3 that the size evolution of initially regular tetrahedra does not offer any additional information in comparison to pair dispersion, I will directly proceed with the analysis of the eigenvalues of the shape tensor. Figure 5.7 shows the evolution of eigenvalues $\left\langle g_{i}(t)\right\rangle$, compensated with the initial edge length squared, as a function of $t / t_{0}$ for polymer concentrations of $5 \mathrm{ppm}$ (a) and $10 \mathrm{ppm}$ (b). For the computation of $t_{0}$, the value for $\varepsilon$ as obtained from the fit to $\langle\delta \mathbf{u} \cdot \delta \mathbf{a}\rangle$ was used again. In figure 5.7, a small difference between the cases with and without polymers can be observed. While the curves for $\left\langle g_{1}(t)\right\rangle$ and $\left\langle g_{3}(t)\right\rangle$ agree within the scatter of the curves, the intermediate eigenvalue suggests that the datasets with polymers (symbols) show a slightly enhanced slope at $t=0$ compared to the datasets without polymers (blue lines), indicating a higher value of $\left\langle s_{2}\right\rangle$.

In order to verify this finding, figure 5.8 shows the strain eigenvalues for datasets with added polymers (symbols) and the average strain eigenvalues for datasets without polymers as obtained from figure 4.1 (blue lines). For the polymer datasets, a slight dependence on $r_{\varepsilon}$ is visible, especially for $10 \mathrm{ppm}$. As discussed earlier, such a small $r / r_{\varepsilon}$ dependence is expected for initial edge lengths at the beginning of the plateau of $\langle\delta \mathbf{u} \cdot \delta \mathbf{a}\rangle$ since $\varepsilon_{p}(r)$ at these scales is non-zero and leads to a deviation of $\langle\delta \mathbf{u} \cdot \delta \mathbf{a}\rangle$ from its final value. As a result, a scale dependent $t_{0}(r)$ arises which becomes smaller for increasing length scales. Since the same value for $t_{0}$ is used for different $l$, however, the absolute values of all eigenvalues $\left\langle s_{i}\right\rangle t_{0}$ seem to grow with increasing scales. Due to this, no horizontal fit for an average value was performed. In the analyzed range of scales, Figure 5.8 (a) confirms that the addition of polymers leads to a higher intermediate eigenvalue $\left\langle s_{2}\right\rangle$, and shows that the absolute values of $\left\langle s_{1}\right\rangle$ and $\left\langle s_{3}\right\rangle$ become smaller. It can be observed that the eigenvalues $\left\langle s_{1}\right\rangle$ and $\left\langle s_{3}\right\rangle$ approach the values of the water case for increasing length scales. This might be a reason why this effect was not observable in figure 5.7. Since the value of $\left\langle s_{1}\right\rangle$ rises slower than $\left\langle s_{3}\right\rangle$ drops, the intermediate eigenvalue $\left\langle s_{2}\right\rangle$ also needs to increase slightly, thus getting further away from the value 0.21 obtained for pure water (see figure 5.8(b)). With $\left\langle s_{1}\right\rangle$ and $\left\langle s_{3}\right\rangle$ 


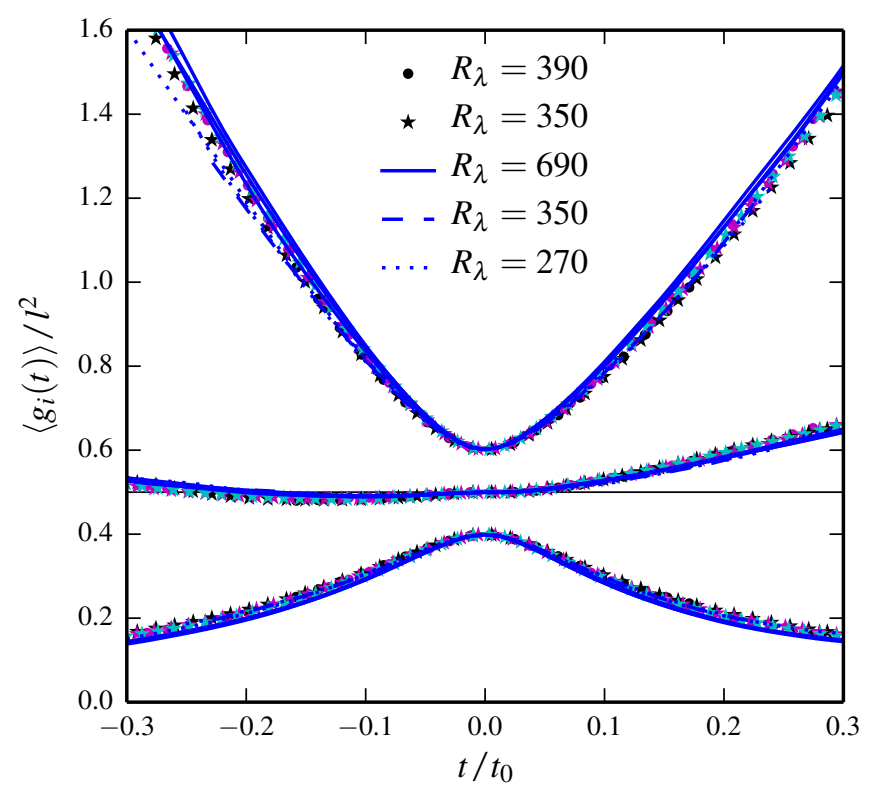

(a) $5 \mathrm{ppm}$

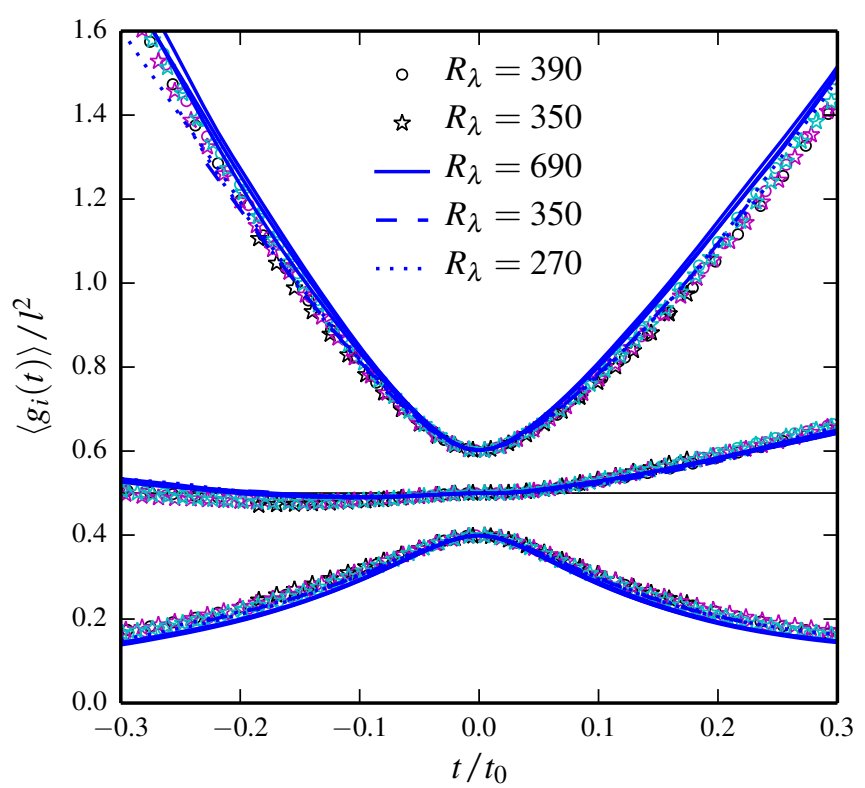

(b) $10 \mathrm{ppm}$

Figure 5.7.: Eigenvalues of the shape tensor, $\mathbf{G}(t)$, obtained from initially nearly regular tetrahedra with edge length $l$. The eigenvalues are non-dimensionalized with $l$, where the variation range of the edge length, $\Delta l / l$, is $10 \%$. Datasets with a polymer concentration of $5 \mathrm{ppm}$ (a) and $10 \mathrm{ppm}$ (b) are shown in comparison to the pure water case. For the polymer datasets, different initial edge lengths of $14 \mathrm{~mm}, 16 \mathrm{~mm}$, and $18 \mathrm{~mm}$ are shown. In terms of the scale $r_{\varepsilon}$ defined in the previous section, the initial edge lengths for a concentration of $5 \mathrm{ppm}$ and $R_{\lambda}=390$ are $l=2.0 r_{\varepsilon}(\bullet)$ and $2.2 r_{\varepsilon}(\bullet)$ and for $R_{\lambda}=350$ they are $l=1.8 r_{\varepsilon}(\star), 2.1 r_{\varepsilon}(\star)$, and $2.4 r_{\varepsilon}(\star)$. For a concentration of $10 \mathrm{ppm}$, the initial edge lengths for $R_{\lambda}=390$ are $l=1.3 r_{\varepsilon}(\circ), 1.5 r_{\varepsilon}(\circ)$, and $1.7 r_{\varepsilon}(\circ)$ and for $R_{\lambda}=350$ they are $l=1.4 r_{\varepsilon}\left(\right.$ 约) $1.6 r_{\varepsilon}$ (约), and $1.8 r_{\varepsilon}$ (放). All water datasets are shown in blue for a better contrast $(-,--, \cdots)$. See figure 4.3 for a color coded version and a listing of the used initial edge lengths. 
approaching the water case, however, the value of $\left\langle s_{2}\right\rangle$ is also bound to return to a value of 0.21 at sufficiently large scales. As a consequence, there needs to be a maximum value of $\left\langle s_{2}\right\rangle$ at some intermediate scale. Furthermore, the close-up in figure 5.8(b) suggests that there might be a dependence of $\left\langle s_{2}\right\rangle$ on the polymer concentration. The large difference between the water and polymer datasets can also be seen more clearly.

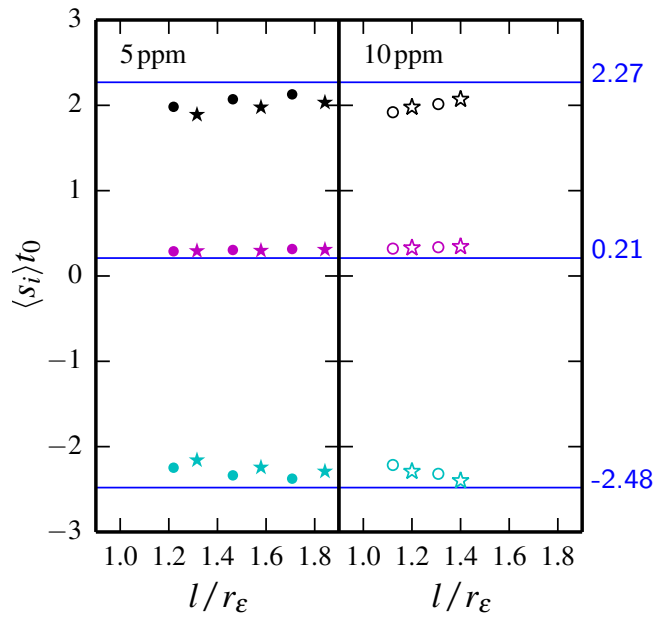

(a) Eigenvalues $\left\langle s_{i}\right\rangle$

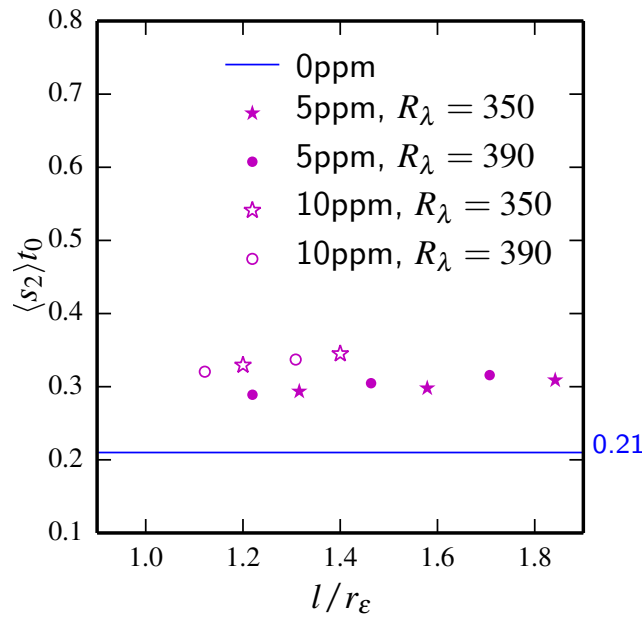

(b) Details of $\left\langle s_{2}\right\rangle$

Figure 5.8.: Eigenvalues of the perceived rate of strain tensor for datasets with added

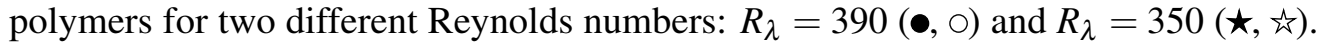
Datasets with a polymer concentration of $5 \mathrm{ppm}$ are shown as filled symbols, datasets with $10 \mathrm{ppm}$ as open symbols. Blue lines indicate the value for the pure water case as obtained from figure 4.1. Only initial separations inside the plateau range of $\langle\delta \mathbf{u} \cdot \delta \mathbf{a}\rangle$ for the small measurement volume are shown. For a list of all values, see appendix B.2.

The observed increase of the intermediate eigenvalue of the perceived rate of strain tensor should also result in an enhancement of the time asymmetry of the evolution of the intermediate shape eigenvalue $\left\langle g_{2}(t)\right\rangle$, as already indicated in figure 5.7. For a better display of this asymmetry, figure 5.9 shows the difference $\left\langle g_{2}(t)-g_{2}(-t)\right\rangle$ compensated with $l^{2}\left(t / t_{0}\right)$. The same datasets as in figure 5.7 are shown. As discussed in section 4.3 , the studied quantity should display plateaus with heights of $2\left\langle s_{2}\right\rangle$. Since a $r_{\varepsilon}$-dependence was observed for the datasets with added polymers, no unique plateau height but rather a range of plateaus is expected. One finds that for all datasets, a steep rise at short times, being a remnant of the fact that the studied tetrahedra are only nearly regular at $t=0$, is followed by the expected stable plateau at larger times. As before, the difference between the pure water case and the polymer cases is apparent. For the datasets with $5 \mathrm{ppm}$ and $10 \mathrm{ppm}$ of added polymers, however, one finds a large scatter of the different curves but no systematic variation with $r_{\varepsilon}$ or polymer concentration. This might be explained with the deviations from a regular shape at $t \approx 0$. It was found in the previous section that there is a strong influence of polymers on the short time evolution of pair dispersion as early as $t \approx 0.01 t_{0}$. 
These early times are very strongly affected by the shape deformations at $t=0$, and possible trends concerning polymer concentration and $r_{\varepsilon}$-dependence could be obscured.

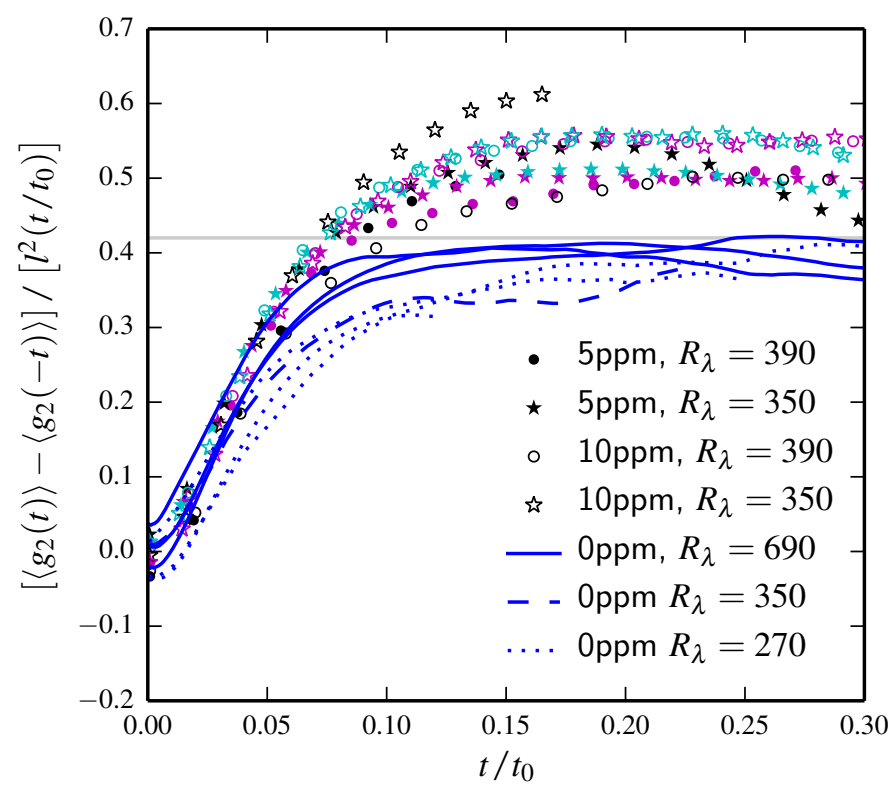

Figure 5.9.: Time asymmetry in the intermediate eigenvalue of the shape tensor for three different polymer concentrations. The same datasets as in figure 5.7 on page 81 are shown. The gray horizontal lines represents the expected plateau height for the water case.

In summary, I find that polymers increase the time-asymmetry in the shape evolution of tetrahedra, specifically in the intermediate eigenvalue of the shape tensor. As for the pair dispersion, a convincing trend that the effect becomes stronger with rising polymer concentration could not be seen. This indicates again that a critical polymer concentration below $5 \mathrm{ppm}$ exists, above which no further changes to the time-asymmetry in particle dispersion can be observed. A slight dependence on $r / r_{\varepsilon}$ was observed, however. Further experimental runs with more tetrahedra trajectories can help to strengthen the results by increasing the overall statistical resolution and, with a stricter bound on the initial deformation of the tetrahedra, by decreasing the deviation from the theoretical prediction at small times. 



\section{CHAPTER 6}

\section{Discussion and Perspectives}

The time irreversibility of turbulent flows is an indicator for their non-equilibrium character, and a better understanding of this connection is important to obtain a complete picture of turbulence. Even though it was already observed a decade ago that pairs of tracer particles separate faster backwards than forwards in time (Sawford et al. 2005, Berg et al. 2006, Bragg et al. 2014), a rigorous explanation for this behavior was not given.

In chapter 3 of this thesis, I showed that the short-time evolution of the relative separation of a pair of tracer particles with an initial separation in the inertial range is given by

$$
\begin{aligned}
\left\langle\delta \mathbf{R}(t)^{2}\right\rangle & =\left\langle\mathbf{V}(0)^{2}\right\rangle t^{2}+\langle\mathbf{V}(0) \cdot \mathbf{A}(0)\rangle t^{3}+\mathcal{O}\left(t^{4}\right) \\
& =\frac{11}{3} C_{2}\left(\varepsilon R_{0}\right)^{\frac{2}{3}} t^{2}-2 \varepsilon t^{3}+\mathcal{O}\left(t^{4}\right),
\end{aligned}
$$

where the first line is a purely kinematic expression, valid for a large range of flows, and the second line corresponds to homogeneous, isotropic turbulence. In the latter case, the time-asymmetric cubic term is found to be directly proportional to the energy flux through scales, $\varepsilon$. For a 3D turbulent flow, the energy cascade goes from large to small scales, leading to a positive value of the energy transfer rate $\varepsilon$ and thus, according to the above formula, to a faster separation backwards than forwards in time. This agrees with the previous findings and was also confirmed in chapter 3 using both experimental and numerical data.

Future studies on the time-asymmetry in pair-dispersion in 2D turbulent flows could provide a good way to further test the presented results. For 2D turbulence, it is known that there exists a range of scales with an inverse energy cascade. In this range, the energy transfer rate is negative, meaning that energy is transported from small to large scales (Kraichnan 1967, Tabeling 2002, Boffetta \& Ecke 2012). For $\varepsilon<0$, the above theoretical relation predicts that the separation of a pair of tracers grows faster forwards than backwards in time, contrary to the 3D case. An observation of this effect would further strengthen the results presented in this thesis. 
Another important conclusion that can be drawn from the above relation is that a clear, time-asymmetric behavior in pair dispersion can already be observed at very short times, long before Richardson scaling is observable. In earlier attempts to explain the observed timeasymmetry, it was stated that at larger times, the relative separation of a particle pair should grow according to Richardson scaling, $\left\langle\delta \mathbf{R}(t)^{2}\right\rangle=g_{f / b} \varepsilon t^{3}$, but with different constants $g_{f}$ and $g_{b}$ for the evolution forwards and backwards in time (Sawford et al. 2005, Berg et al. 2006, Salazar \& Collins 2009). Plotting the difference between the evolution backwards and forwards in time and dividing by $\varepsilon t^{3}$ should thus lead to two plateaus: For the short-time evolution one expects a plateau with height 4 , while for later times a plateau with a height of $\left(g_{b}-g_{f}\right)$ should be visible. Berg et al. (2006) extracted a value of $\left(g_{b}-g_{f}\right)=0.6$ from their experimental data at $R_{\lambda}=172$ by using a controversial time-shifting procedure (Ouellette 2006, pp. 144-145). Even though the Reynolds numbers used in this thesis are much larger, all presented experimental and numerical datasets only showed a clear plateau for the shorttime evolution and then quickly dropped below a value of 0.6 without the indication of a second plateau. It cannot be fully ruled out however, that a second plateau at a much smaller value of $\left(g_{b}-g_{f}\right)$ might exist. Further experiments with longer observation times are needed to reliably test for the existence of a second plateau at later times.

While the symmetry-breaking term in pair dispersion was found to behave like $t^{3}$, the time-symmetry in the shape evolution of clusters of four particles is already broken by a term linear in time. Using the perceived rate of strain tensor as defined by Chertkov et al. (1999), I showed in chapter 4 that the evolution of the eigenvalues of the shape tensor can be expressed by

$$
\begin{aligned}
\left\langle g_{i}(t)\right\rangle=\frac{l^{2}}{2}\left(1+2\left\langle s_{i}\right\rangle t+\right. & \left\langle S_{1, i i}+2 s_{i}^{2}\right\rangle t^{2}+\left\langle\frac{1}{3} S_{2, i i}+\frac{4}{3} s_{i}^{3}+2\left(\mathbf{S}_{0} \cdot \mathbf{S}_{1}\right)_{i i}\right. \\
& \left.\left.+\frac{2}{3}\left(\mathbf{R}_{0}^{2} \cdot \mathbf{S}_{0}\right)_{i i}-\frac{2}{3}\left(\mathbf{R}_{0} \cdot \mathbf{S}_{0} \cdot \mathbf{R}_{0}\right)_{i i}+\frac{4}{3}\left(\mathbf{R}_{0} \cdot \mathbf{S}_{1}\right)_{i i}\right\rangle t^{3}+\mathcal{O}\left(t^{4}\right)\right),
\end{aligned}
$$

where both the eigenvalues of the shape tensor, $\left\langle g_{i}(t)\right\rangle$, and the eigenvalues of the perceived rate of strain tensor, $\left\langle s_{i}\right\rangle$, are sorted by size. Here, the symmetry-breaking, linear term is proportional to the eigenvalues of the perceived rate of strain tensor which, similarly to the true rate of strain tensor, obey $\left\langle s_{1}\right\rangle>\left\langle s_{2}\right\rangle>\geq 0>\left\langle s_{3}\right\rangle$. If the intermediate eigenvalue equals zero, as it is e.g. the case for a Gaussian flow field, the shape evolution is time-symmetric. For the turbulent flow and the range of tetrahedra sizes studied in this thesis, however, the intermediate eigenvalue was found to have a value of $\left\langle s_{2}\right\rangle t_{0}=0.21$, with $t_{0}$ being the timescale corresponding to the size of the tetrahedron. The predicted asymmetry of $\left\langle g_{i}(t)-g_{i}(-t)\right\rangle=$ $2 l^{2}\left\langle s_{2}\right\rangle t$ was observed with experimental and numerical data for intermediate times. For very short times, the fact that the experimentally observed tetrahedra are not perfectly regular, as was assumed in the theoretical derivation, obscures the effect. Only a drastic increase in tracked tetrahedra can alleviate this problem by allowing a stricter selection of the initial shape at $t=0$. Further high-precision experiments are therefore desirable.

Another attractive continuation of this work is the study of single-particle statistics. While simple Lagrangian velocity increments of the type $\left\langle(\mathbf{U}(t+\tau \mid \mathbf{y}, 0)-\mathbf{U}(t \mid \mathbf{y}, 0))^{n}\right\rangle$ can be shown to be invariant under time reversal (Falkovich et al. 2012), it was recently found that the 
kinetic energy of single tracer particles indeed captures the irreversibility of the flow (Xu et al. 2014). Furthermore, the same behavior was observed for 2D and 3D turbulence. This implies that the time-asymmetry observed in single-particle statistics might be directly connected to energy dissipation, and not, as it was shown for multi-particle statistics in this thesis, to the flux of energy through scales. It will be of great interest to find the relation and differences between the time irreversibility observed in single- and multi-particle dispersion.

In chapter 5, I lastly studied what effect a change to the energy cascade, induced by the addition of small amounts of polyacrylamide, might have on the time-asymmetry in the above processes. Since polymers not only influence the bulk flow but also hamper the energy injection into the flow due to drag reduction effects at the propellers, for each dataset with added polymers, the energy transfer rate was obtained by a fit to the plateau of $\langle\boldsymbol{\delta} \mathbf{u} \cdot \boldsymbol{\delta} \mathbf{a}\rangle$. I then confirmed that the length scale $r_{\varepsilon}$, introduced by Xi et al. (2013), at which the turbulence energy flux and the elastic energy flux through the polymers are equally strong, is able to collapse all datasets with added polymers onto a single curve. Looking at the dispersion of two particles and rescaling everything with the measured value of $\varepsilon$, a distinct difference between the water and polymer datasets could be observed whereas the results for two different polymer concentrations, $5 \mathrm{ppm}$ and $10 \mathrm{ppm}$, agreed within the uncertainty of the data. This implies that there is a critical polymer concentration smaller than $5 \mathrm{ppm}$ above which different polymer concentrations lead to the same effect, as long as everything is normalized correctly and the chosen initial separations are in the inertial range. Furthermore, the main difference between the water and polymer cases consisted of an earlier deviation from the $t^{3}$ scaling. I argued that this early deviation could be caused by the introduction of a new time scale which affects the higher order terms.

For the shape deformation of tetrahedra under the influence of added polymers, I found a significant enhancement of the time-asymmetry for the dilute polymer solutions. For the two different polymer concentrations, a small difference in the intermediate eigenvalue of the perceived rate of strain tensor was observed. This difference was not reflected in the asymmetry shown by $\left\langle g_{i}(t)-g_{i}(-t)\right\rangle$, however. Under the assumption that polymers indeed introduce a new time scale, the expected effect should be visible at small times. As discussed before, the shape evolution at very short times is again obscured by the small deformations of the tetrahedra at $t=0$. Further measurements with a stricter shape selection at $t=0$ can help to increase the non-obscured range of scales and help to investigate the existence of a new time scale. Additionally, experiments at different polymer concentrations, especially below the supposed critical concentration of approximately $5 \mathrm{ppm}$, are needed to clarify the existence of a critical concentration and its exact value.

In summary, the theoretical and experimental results obtained in this thesis open new ways to investigate turbulent flows and reveal many new questions to be answered. 

Appendix

\section{A. Supplementary Figures and Derivations}

This appendix contains supplemental plots as well as detailed versions of arguments and derivations that are indispensable parts of this thesis but were moved to the appendix for better readability of the main text.

\section{A.1. Measuring $\varepsilon$ for $R_{\lambda}=270$}

The figure below shows $\langle\delta \mathbf{u} \cdot \delta \mathbf{a}\rangle$ for three different polymer concentrations at $R_{\lambda}=270$ (a) and for comparison also at $R_{\lambda}=350$ (b). Horizontal lines show the fits to obtain the energy transfer rate. While nice plateaus can be seen for all three datasets with the higher Reynolds number in figure (b), the datasets with the lower Reynolds number show only very short plateaus. For the pure water case, the plateau is very noisy and, since the Kolmogorov length scale $\eta$ is very large for this dataset $(\eta=104 \mu \mathrm{m})$, the fit extends into the range where the influence due to the finite volume of the measurement volume interferes with it (approximately $r \gtrsim 10 \mathrm{~mm}$ ). However, one can argue that the obtained value of $\varepsilon$ should remain valid with only a slightly increased uncertainty. Adding polymers however, the plateau is shifted towards larger scales (as explained in section 5.2) making the influence of the finite measurement volume more dominant. It is not certain that the true plateau height is ever reached before the diminishing effect of the finite observation volume takes over. The datasets at $R_{\lambda}=270$ with added polymers will thus only be used in cases where the measured value of $\varepsilon$ does not have a significant impact. 


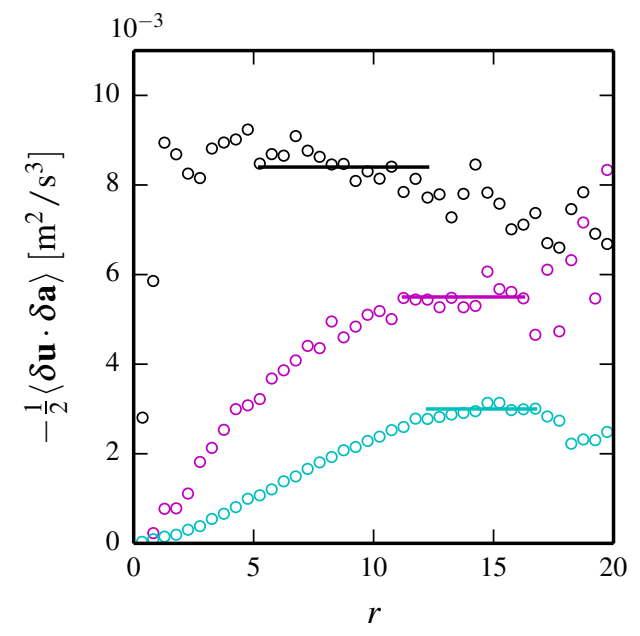

(a) $R_{\lambda}=270$

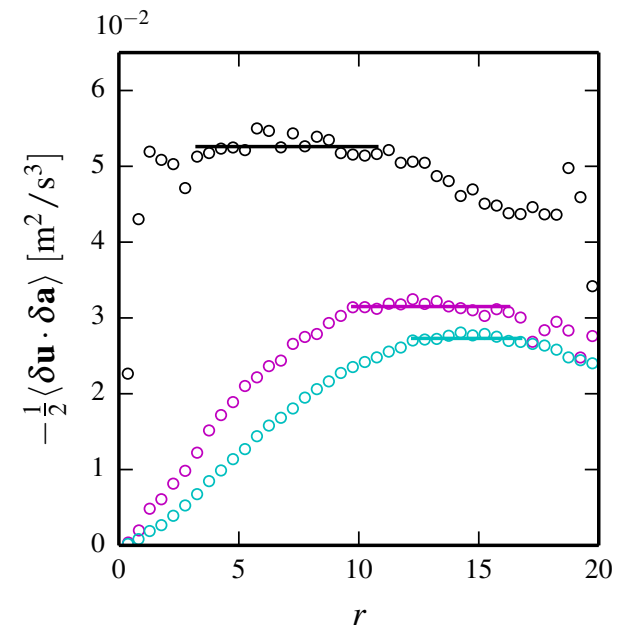

(b) $R_{\lambda}=350$

Figure A.1.: Fits to obtain the energy transfer rate $\varepsilon$ from $\langle\delta \mathbf{u} \cdot \delta \mathbf{a}\rangle=-2 \varepsilon$ for two different Reynolds numbers: $R_{\lambda}=270 \leftrightarrow 0.5 \mathrm{~Hz}$ (a) and $R_{\lambda}=350 \leftrightarrow 0.9 \mathrm{~Hz}$. Three different polymer concentrations are shown with black circles for $0 \mathrm{ppm}$ (pure water), magenta circles for $5 \mathrm{ppm}$, and cyan circles for $10 \mathrm{ppm}$. Fits are indicated by horizontal lines.

\section{A.2. Convergence Radius of the Short-Time Expansion for the Mean Squared Change of Separation}

This appendix presents a more detailed version of a proof by Anna Frishman from the Weizmann Institute of Science, Israel (Frishman 2013). It is founded on many fruitful discussions we had about this topic.

The Taylor expansion for the mean squared change of separation around $t=0$, as shown in equation (3.1), can be written in a more systematic form as

$$
\begin{aligned}
\left\langle\delta \mathbf{R}(t)^{2}\right\rangle=\left[2\left\langle\mathbf{V}(0)^{2}\right\rangle\right]_{t=0} \frac{t^{2}}{2}+\left[3 \frac{\mathrm{d}}{\mathrm{d} t}\left\langle\mathbf{V}(t)^{2}\right\rangle\right]_{t=0} \frac{t^{3}}{6} & \\
+ & {\left[8 \frac{\mathrm{d}}{\mathrm{d} t}\left\langle\mathbf{V}(t) \cdot \frac{\mathrm{d} \mathbf{V}(t)}{\mathrm{d} t}\right\rangle-2\left\langle\left(\frac{\mathrm{d} \mathbf{V}(t)}{\mathrm{d} t}\right)^{2}\right\rangle\right]_{t=0} \frac{t^{4}}{24} } \\
+ & {\left[5 \frac{\mathrm{d}}{\mathrm{d} t}\left\langle\left(\frac{\mathrm{d} \mathbf{V}(t)}{\mathrm{d} t}\right)^{2}\right\rangle+10 \frac{\mathrm{d}}{\mathrm{d} t}\left\langle\mathbf{V}(t) \cdot \frac{\mathrm{d}^{2} \mathbf{V}(t)}{\mathrm{d} t^{2}}\right\rangle\right]_{t=0} \frac{t^{5}}{120}+\mathcal{O}\left(t^{6}\right) }
\end{aligned}
$$

where the first five orders have been computed explicitly. Here, $\mathbf{V}(t)$ is the relative velocity between the particles. In order to determine the radius of convergence of this series, it is educating to first investigate which time scales can appear. It is intuitively clear that for any two-point function, a function depending on the separation $\mathbf{R}$ between two points, the Kolmogorov time $t_{0}$ of an eddy of this size is the relevant time scale. Another quantity that may appear is $\tau_{\eta}$, which is the defining time scale of spatial derivatives and which can be 
several orders of magnitude smaller than $t_{0}$. In equation (A.1), $\tau_{\eta}$ enters through the squared relative acceleration $\left\langle(\mathrm{d} \mathbf{V}(t) / \mathrm{d} t)^{2}\right\rangle$ in the $t^{4}$ term. Using the Navier-Stokes equations, it can be shown that this correlation function contains expressions only depending on the spatial derivatives of single-particle velocities, thus introducing $\tau_{\eta}$. The same happens for many higher order terms, therefore reducing the convergence radius of the full series to approximately $\tau_{\eta}$. One can show, however, that a series of only the odd terms in equation (A.1) has a much larger convergence radius of approximately $t_{0}$. For this purpose, we will show that the odd terms do not contain single-point functions.

Let us assume we have a statistical quantity that does not explicitly depend on the particle separation $\mathbf{R}(t)$,

$$
\begin{aligned}
& \left\langle F\left[\mathbf{V}(t), \frac{\mathrm{d}}{\mathrm{d} t} \mathbf{V}(t), \ldots\right]\right\rangle \\
& \quad=\int P_{\mathbf{R}(t)}\left[\mathbf{V}(t), \frac{\mathrm{d}}{\mathrm{d} t} \mathbf{V}(t), \ldots\right] F\left[\mathbf{V}(t), \frac{\mathrm{d}}{\mathrm{d} t} \mathbf{V}(t), \ldots\right] \mathrm{d} \mathbf{V}(t) \mathrm{d}\left(\frac{\mathrm{d}}{\mathrm{d} t} \mathbf{V}(t)\right) \ldots
\end{aligned}
$$

There still persists an implicit dependence on $\mathbf{R}(t)$ through the p.d.f. of the velocity derivatives, $P_{\mathbf{R}(t)}\left[\mathbf{V}(t), \frac{\mathrm{d}}{\mathrm{d} t} \mathbf{V}(t), \ldots\right]$. For a stationary, incompressible and homogeneous flow, the full time derivative at $t=0$ for such a quantity can be written as

$$
\left.\frac{\mathrm{d}}{\mathrm{d} t}\left\langle F\left[\mathbf{V}(t), \frac{\mathrm{d}}{\mathrm{d} t} \mathbf{V}(t), \ldots\right]\right\rangle\right|_{t=0}=\left.\sum_{i=1}^{3} \frac{\mathrm{d}}{\mathrm{d} R_{i}(t)}\left\langle V_{i}(t) F\left[\mathbf{V}(t), \frac{\mathrm{d}}{\mathrm{d} t} \mathbf{V}(t), \ldots\right]\right\rangle\right|_{t=0}
$$

This means, that even for the case where $F\left(\mathbf{V}, \frac{\mathrm{d}}{\mathrm{d} t} \mathbf{V}, \ldots\right)$ contains contributions from single point functions, they will not contribute to the time derivative since they are, by definition, independent of $\mathbf{R}(t)$. As a consequence, any term in equation (A.1) which can be expressed solely by total time derivatives is free of single point functions and thus introduces a time scale $t_{0}$ and not $\tau_{\eta}$. At a first glance, it can be seen that this is both true for the $t^{3}$ and the $t^{5}$ term, and we can show analytically that this is also true for any other odd term. For the $n$th term in equation (A.1), the prefactor is given by

$$
\begin{aligned}
\left.\frac{\mathrm{d}^{n}}{\mathrm{~d} t^{n}}\left\langle\delta \mathbf{R}(t)^{2}\right\rangle\right|_{t=0}= & \left.2 \frac{\mathrm{d}^{n-1}}{\mathrm{~d} t^{n-1}}\langle\delta \mathbf{R}(t) \cdot \mathbf{V}(t)\rangle\right|_{t=0} \\
= & \left.2 \frac{\mathrm{d}^{n-2}}{\mathrm{~d} t^{n-2}}\left\langle\mathbf{V}(t)^{2}\right\rangle\right|_{t=0}+\left.2 \frac{\mathrm{d}^{n-2}}{\mathrm{~d} t^{n-2}}\left\langle\delta \mathbf{R}(t) \cdot \frac{\mathrm{d}}{\mathrm{d} t} \mathbf{V}(t)\right\rangle\right|_{t=0} \\
= & \left.2 \frac{\mathrm{d}^{n-2}}{\mathrm{~d} t^{n-2}}\left\langle\mathbf{V}(t)^{2}\right\rangle\right|_{t=0}+\left.2 \frac{\mathrm{d}^{n-3}}{\mathrm{~d} t^{n-3}}\left\langle\mathbf{V}(t) \cdot \frac{\mathrm{d}}{\mathrm{d} t} \mathbf{V}(t)\right\rangle\right|_{t=0} \\
& \quad+\left.2 \frac{\mathrm{d}^{n-3}}{\mathrm{~d} t^{n-3}}\left\langle\delta \mathbf{R}(t) \cdot \frac{\mathrm{d}^{2}}{\mathrm{~d} t^{2}} \mathbf{V}(t)\right\rangle\right|_{t=0} \cdot \\
= & \left.2 \frac{\mathrm{d}^{n-2}}{\mathrm{~d} t^{n-2}}\left\langle\mathbf{V}(t)^{2}\right\rangle\right|_{t=0}+\left.2 \frac{\mathrm{d}^{n-3}}{\mathrm{~d} t^{n-3}}\left\langle\mathbf{V}(t) \cdot \frac{\mathrm{d}}{\mathrm{d} t} \mathbf{V}(t)\right\rangle\right|_{t=0}+\ldots \\
& \quad+\left.\frac{\mathrm{d}}{\mathrm{d} t}\left\langle\mathbf{V}(t) \cdot \frac{\mathrm{d}^{n-3}}{\mathrm{~d} t^{n-3}} \mathbf{V}(t)\right\rangle\right|_{t=0}+\left.\frac{\mathrm{d}}{\mathrm{d} t}\left\langle\delta \mathbf{R}(t) \cdot \frac{\mathrm{d}^{n-2}}{\mathrm{~d} t^{n-2}} \mathbf{V}(t)\right\rangle\right|_{t=0}
\end{aligned}
$$

It can be seen that all but the very last term are already in the form of equation (A.3), meaning 
that they do not introduce single-point functions. The last term apparently still depends on $\delta \mathbf{R}(t)$ and thus needs further investigation. Taking the final derivative, one finds

$$
\left.\frac{\mathrm{d}}{\mathrm{d} t}\left\langle\delta \mathbf{R}(t) \cdot \frac{\mathrm{d}^{n-2}}{\mathrm{~d} t^{n-2}} \mathbf{V}(t)\right\rangle\right|_{t=0}=\left.\left\langle\mathbf{V}(t) \cdot \frac{\mathrm{d}^{n-2}}{\mathrm{~d} t^{n-2}} \mathbf{V}(t)\right\rangle\right|_{t=0}+\left.\left\langle\delta \mathbf{R}(t) \cdot \frac{\mathrm{d}^{n-1}}{\mathrm{~d} t^{n-1}} \mathbf{V}(t)\right\rangle\right|_{t=0}
$$

where the second term vanishes for $t=0$ since $\delta \mathbf{R}(0)=0$ by definition. It remains to explore if the first term can be written as a total derivative according to equation (A.3). Using the product rule, one finds

$$
\left\langle\mathbf{V}(t) \cdot \frac{\mathrm{d}^{n-2}}{\mathrm{~d} t^{n-2}} \mathbf{V}(t)\right\rangle=\frac{\mathrm{d}}{\mathrm{d} t}\left\langle\mathbf{V}(t) \cdot \frac{\mathrm{d}^{n-3}}{\mathrm{~d} t^{n-3}} \mathbf{V}(t)\right\rangle-\left\langle\frac{\mathrm{d}}{\mathrm{d} t} \mathbf{V}(t) \cdot \frac{\mathrm{d}^{n-3}}{\mathrm{~d} t^{n-3}} \mathbf{V}(t)\right\rangle,
$$

where the product rule can be applied again to the last term and so forth. For $n$ being even, this process finds an end when the last term has the form

$$
\left\langle\left(\frac{\mathrm{d}^{(n-2) / 2}}{\mathrm{~d} t^{(n-2) / 2}} \mathbf{V}(t)\right)^{2}\right\rangle
$$

which can not be rewritten as a total derivative. For $n$ being odd however, at the end of this procedure the last term is given by

$$
\left\langle\frac{\mathrm{d}^{(n-1) / 2-1}}{\mathrm{~d} t^{(n-1) / 2-1}} \mathbf{V}(t) \cdot \frac{\mathrm{d}^{(n-1) / 2}}{\mathrm{~d} t^{(n-1) / 2}} \mathbf{V}(t)\right\rangle=\frac{1}{2} \frac{\mathrm{d}}{\mathrm{d} t}\left\langle\left(\frac{\mathrm{d}^{(n-1) / 2}}{\mathrm{~d} t^{(n-1) / 2}} \mathbf{V}(t)\right)^{2}\right\rangle,
$$

which has the form (A.3). As a result we find that every odd term in the expansion in equation (A.1) can be written exclusively in terms of total derivatives of the form (A.3), so that an expansion of only the odd terms must have a radius of convergence of the order of $t_{0}$. 


\section{A.3. Elusive Richardson Scaling in Pair Dispersion}

This figure shows the same data as figure 3.7, with the only difference that for the experimental datasets, all points with more than $10^{4}$ observed pairs are shown (instead of $10^{6}$ ) in order to observe the evolution at later times. It can be seen that the data drops rapidly towards zero and always stays below the numerical datasets.

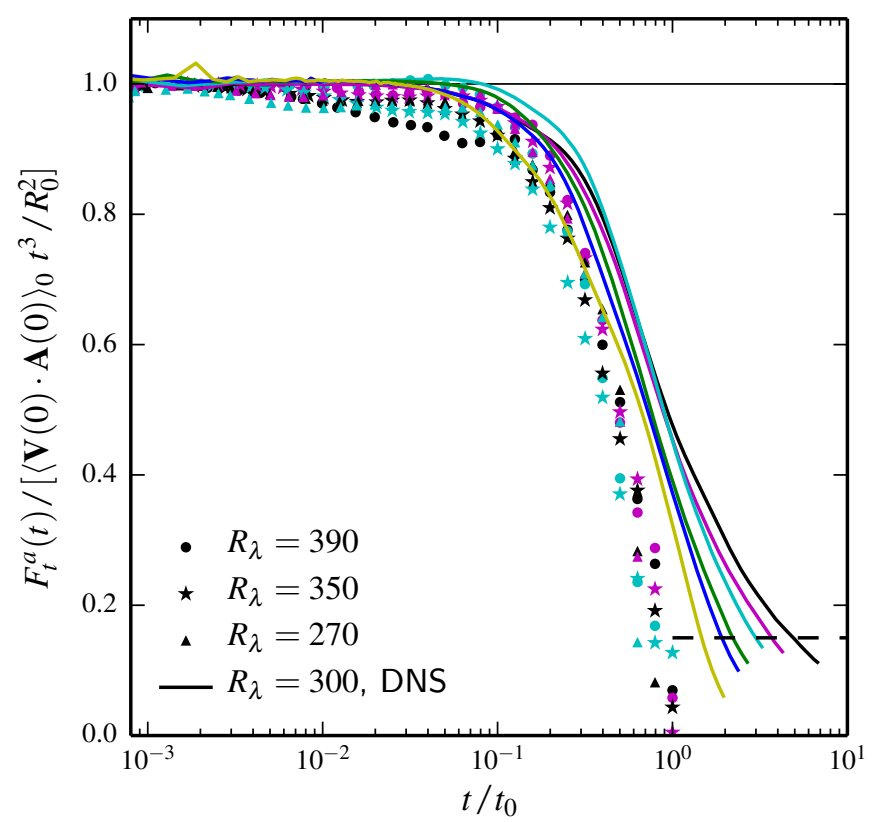

Figure A.2.: Experimental and numerical results for the odd terms of the compensated mean squared change of separation. For experimental data at $R_{\lambda}=390$, the initial separations are $241 \eta(\bullet), 276 \eta(\bullet)$ and $310 \eta(\bullet)$. For $R_{\lambda}=350$, the initial separations are $212 \eta$ $(\star), 242 \eta(\star)$ and $273 \eta(\star)$, and for $R_{\lambda}=270$, they are $135 \eta(\boldsymbol{\Delta}), 154 \eta(\boldsymbol{\Delta})$ and $173 \eta(\Delta)$. For the DNS data at $R_{\lambda}=300$, the initial separations are $19 \eta(-), 38 \eta$ (一), $58 \eta(-), 77 \eta(-), 92 \eta(-)$ and $123 \eta(-)$. The dashed horizontal line indicates the prediction by Richardson scaling as proposed by Berg et al. (2006). 


\section{A.4. Diagonal Form of the Shape Tensor}

Following section 4.1, the short time evolution of the shape tensor is given by

$$
\begin{aligned}
\mathbf{G}(t)= & \frac{l^{2}}{2}\left(\mathbb{1}+2 \mathbf{S}_{0} t+\left(2 \mathbf{S}_{1}+4 \mathbf{S}_{0}^{2}+2\left[\mathbf{S}_{0}, \Omega_{0}\right]\right) t^{2}+\left(2 \mathbf{S}_{2}+8 \mathbf{S}_{0}^{3}+6\left[\mathbf{S}_{0}^{2}, \Omega_{0}\right]\right.\right. \\
& \left.\left.+2\left[\left[\mathbf{S}_{0}, \Omega_{0}\right], \Omega_{0}\right]+6 \mathbf{S}_{0} \cdot \mathbf{S}_{1}+6 \mathbf{S}_{1} \cdot \mathbf{S}_{0}+2\left[\mathbf{S}_{1}, \Omega_{0}\right]+4\left[\mathbf{S}_{0}, \Omega_{1}\right]\right) t^{3}+\mathcal{O}\left(t^{4}\right)\right)
\end{aligned}
$$

With the rotation matrix $\mathbf{U}(t)$, the shape tensor can be diagonalized as

$$
\mathbf{G}^{D}(t)=\mathbf{U}(t) \mathbf{G}(t) \mathbf{U}^{T}(t)=\text { diagonal. }
$$

Using that rotation matrices are orthogonal, $\mathbf{U}(t) \mathbf{U}^{T}(t)=\mathbb{1}$, one finds $\frac{\mathrm{d}}{\mathrm{d} t} \mathbf{U}(t)=\mathbf{R}(t) \mathbf{U}(t)$ with $\mathbf{R}^{T}(t)=-\mathbf{R}(t)$. Expanding $\mathbf{U}(t)$ in a Taylor series around $t=0$ and expressing it through the antisymmetric matrix $\mathbf{R}(t)$ leads to

$$
\mathbf{U}(t)=\left[\mathbb{1}+\mathbf{R}_{0} t+\left(\mathbf{R}_{1}+\mathbf{R}_{0}^{2}\right) \frac{t^{2}}{2}+\left(\mathbf{R}_{2}+2 \mathbf{R}_{1} \cdot \mathbf{R}_{0}+\mathbf{R}_{0} \cdot \mathbf{R}_{1}+\mathbf{R}_{0}^{3}\right) \frac{t^{3}}{6}+\mathcal{O}\left(t^{4}\right)\right] \mathbf{U}_{0} .
$$

where $\mathbf{R}_{n}=\left.\frac{\mathrm{d}^{n}}{\mathrm{~d} t^{n}} \mathbf{R}(t)\right|_{t=0}$ with $\mathbf{R}_{n}^{T}=-\mathbf{R}_{n}$ for all $n$. I again use the short notation for the Taylor expansions,

$$
\begin{aligned}
\mathbf{G}(t) & =\mathbf{G}_{0}+\mathbf{G}_{1} t+\mathbf{G}_{2} \frac{t^{2}}{2}+\mathbf{G}_{3} \frac{t^{3}}{6}+\mathcal{O}\left(t^{4}\right) \\
\text { and } \mathbf{G}^{D}(t) & =\mathbf{G}_{0}^{D}+\mathbf{G}_{1}^{D} t+\mathbf{G}_{2}^{D} \frac{t^{2}}{2}+\mathbf{G}_{3}^{D} \frac{t^{3}}{6}+\mathcal{O}\left(t^{4}\right),
\end{aligned}
$$

and insert them together with equation (A.11) into equation (A.10). Below, each order in $t$ is analyzed separately to obtain the missing $\mathbf{G}_{n}$. Thereby, all appearing terms of the type $\mathbf{R}_{n} \mathbf{U}_{0} \mathbb{1} \mathbf{U}_{0}^{T}+\mathbf{U}_{0} \mathbb{1} \mathbf{U}_{0}^{T} \mathbf{R}_{n}^{T}=\mathbf{R}_{n}+\mathbf{R}_{n}^{T}=0$ are directly omitted.

\section{Zeroth Order}

$$
\mathbf{G}_{0}^{D}=\mathbf{U}_{0} \mathbf{G}_{0} \mathbf{U}_{0}^{T}=\mathbf{U}_{0} \frac{l^{2}}{2} \mathbb{1} \mathbf{U}_{0}^{T}=\frac{l^{2}}{2} \mathbf{U}_{0} \mathbf{U}_{0}^{T}=\frac{l^{2}}{2} \mathbb{1}
$$

First Order

$$
\mathbf{G}_{1}^{D}=\mathbf{U}_{0} \mathbf{G}_{1} \mathbf{U}_{0}^{T}=l^{2} \mathbf{U}_{0} \mathbf{S}_{0} \mathbf{U}_{0}^{T} .
$$

Here, $\mathbf{U}_{0}$ must be chosen such that $\mathbf{G}_{1}^{D}$ is diagonal, which corresponds to a rotation into the eigenframe of $\mathbf{S}_{0}$. In all higher order terms discussed below, each term is framed by $\mathbf{U}_{0}$ and $\mathbf{U}_{0}^{T}$. Therefore, I will omit $\mathbf{U}_{0}$ from now on and expect every term to be understood in the eigenframe of $\mathbf{S}_{0}$. 
Second Order

$$
\begin{aligned}
\mathbf{G}_{2}^{D} & =\mathbf{G}_{2}-2\left[\mathbf{G}_{1}, \mathbf{R}_{0}\right] \\
& =\frac{l^{2}}{2}\left(2 \mathbf{S}_{1}+4 \mathbf{S}_{0}^{2}+2\left[\mathbf{S}_{0}, \Omega_{0}\right]\right)-4 \frac{l^{2}}{2}\left[\mathbf{S}_{0}, \mathbf{R}_{0}\right]
\end{aligned}
$$

where $\left[\mathbf{S}_{0}, \mathbf{R}_{0}\right]=\mathbf{S}_{0} \cdot \mathbf{R}_{0}-\mathbf{R}_{0} \cdot \mathbf{S}_{0}$ is purely off-diagonal. With the right choice of $\mathbf{R}_{0}$, this term can thus be used to cancel any off-diagonal terms in equation (A.16). Since all matrices are given in the eigenframe of $\mathbf{S}_{0}$, one finds that $\mathbf{S}_{0}^{2}$ is diagonal whereas $\left[\mathbf{S}_{0}, \Omega_{0}\right]$ is purely off-diagonal. The derivative of the perceived rate of strain tensor, $\mathbf{S}_{1}$, can have diagonal and off-diagonal terms. Denoting the diagonal part of a general matrix $\mathbf{A}$ by $\mathcal{D}(\mathbf{A})$, noting that $\mathcal{D}\left(\mathbf{A}^{T}\right)=\mathcal{D}(\mathbf{A})$, the condition on $\mathbf{R}_{0}$ for $\mathbf{G}_{2}^{D}$ to be diagonal is given by

$$
4\left[\mathbf{S}_{0}, \mathbf{R}_{0}\right]=2\left(\mathbf{S}_{1}-\mathcal{D}\left(\mathbf{S}_{1}\right)\right)+2\left[\mathbf{S}_{0}, \Omega_{0}\right] .
$$

Choosing $\mathbf{R}_{0}$ such that equation (A.17) is fulfilled, the second order for the diagonalized shape tensor is found to be

$$
\mathbf{G}_{2}^{D}=l^{2} \mathcal{D}\left(\mathbf{S}_{1}\right)+2 l^{2} \mathbf{S}_{0}^{2} .
$$

\section{Third Order}

$$
\begin{aligned}
\mathbf{G}_{3}^{D} & =\mathbf{G}_{3}+3 \mathbf{R}_{0} \cdot \mathbf{G}_{2}+3 \mathbf{G}_{2} \cdot \mathbf{R}_{0}^{T}+6 \mathbf{R}_{0} \cdot \mathbf{G}_{1} \cdot \mathbf{R}_{0}^{T}+3\left(\mathbf{R}_{1}+\mathbf{R}_{0}^{2}\right) \cdot \mathbf{G}_{1}+3 \mathbf{G}_{1} \cdot\left(\mathbf{R}_{1}+\mathbf{R}_{0}^{2}\right)^{T} \\
& =\mathbf{G}_{3}-3\left[\mathbf{G}_{2}, \mathbf{R}_{0}\right]-3\left[\mathbf{G}_{1}, \mathbf{R}_{1}\right]+3\left(\mathbf{R}_{0}^{2} \cdot \mathbf{G}_{1}+\mathbf{G}_{1} \cdot \mathbf{R}_{0}^{2}-2 \mathbf{R}_{0} \cdot \mathbf{G}_{1} \cdot \mathbf{R}_{0}\right) .
\end{aligned}
$$

Since $\mathbf{G}_{1}$ is diagonal in the eigenframe of $\mathbf{S}_{0}$ and $\mathbf{R}_{1}$ is antisymmetric, the commutator $\left[\mathbf{G}_{1}, \mathbf{R}_{1}\right]$ is purely off-diagonal and can be used to eliminate all other off-diagonal terms. Similar to equation (A.17), a condition for $\mathbf{R}_{1}$ can be derived. I will omit this step here and just assume this condition to be fulfilled. One then finds that

$$
\mathbf{G}_{3}^{D}=\mathcal{D}\left(\mathbf{G}_{3}\right)-3 \mathcal{D}\left(\left[\mathbf{G}_{2}, \mathbf{R}_{0}\right]+3 \mathcal{D}\left(\mathbf{R}_{0}^{2} \cdot \mathbf{G}_{1}+\mathbf{G}_{1} \cdot \mathbf{R}_{0}^{2}-2 \mathbf{R}_{0} \cdot \mathbf{G}_{1} \cdot \mathbf{R}_{0}\right) .\right.
$$

In more detail, the first term is given by

$\mathcal{D}\left(\mathbf{G}_{3}\right)=\frac{l^{2}}{2}\left(2 \mathcal{D}\left(\mathbf{S}_{2}\right)+8 \mathbf{S}_{0}^{3}+4 \mathcal{D}\left(\mathbf{S}_{0} \cdot \Omega_{0}^{2}\right)-4 \mathcal{D}\left(\Omega_{0} \cdot \mathbf{S}_{0} \cdot \Omega_{0}\right)+12 \mathcal{D}\left(\mathbf{S}_{0} \cdot \mathbf{S}_{1}\right)+4 \mathcal{D}\left(\mathbf{S}_{1} \cdot \Omega_{0}\right)\right)$, and for the second term one finds

$$
\mathcal{D}\left(\left[\mathbf{G}_{2}, \mathbf{R}_{0}\right]\right)=\frac{l^{2}}{2}\left(\mathcal{D}\left(\left[\mathbf{G}_{2}^{D}, \mathbf{R}_{0}\right]\right)+4 \mathcal{D}\left(\left[\left[\mathbf{S}_{0}, \mathbf{R}_{0}\right], \mathbf{R}_{0}\right]\right)=8 \mathcal{D}\left(\mathbf{R}_{0}^{2} \cdot \mathbf{S}_{0}\right)-8 \mathcal{D}\left(\mathbf{R}_{0} \cdot \mathbf{S}_{0} \cdot \mathbf{R}_{0}\right)\right),
$$


where I used at the second equality that $\left[\mathbf{G}_{2}^{D}, \mathbf{R}_{0}\right]$ is purely off-diagonal. The last term in equation (A.20) finally yields

$$
\mathcal{D}\left(\mathbf{R}_{0}^{2} \cdot \mathbf{G}_{1}+\mathbf{G}_{1} \cdot \mathbf{R}_{0}^{2}-2 \mathbf{R}_{0} \cdot \mathbf{G}_{1} \cdot \mathbf{R}_{0}\right)=\frac{l^{2}}{2}\left(4 \mathcal{D}\left(\mathbf{R}_{0}^{2} \cdot \mathbf{S}_{0}\right)-4 \mathcal{D}\left(\mathbf{R}_{0} \cdot \mathbf{S}_{0} \cdot \mathbf{R}_{0}\right)\right) .
$$

The complete third order term of the diagonalized shape tensor is thus given by

$$
\begin{aligned}
\mathbf{G}_{3}^{D}= & \frac{l^{2}}{2}\left(2 \mathcal{D}\left(\mathbf{S}_{2}\right)+8 \mathbf{S}_{0}^{3}+12 \mathcal{D}\left(\mathbf{S}_{0} \cdot \mathbf{S}_{1}\right)+4 \mathcal{D}\left(\mathbf{S}_{0} \cdot \Omega_{0}^{2}\right)-4 \mathcal{D}\left(\Omega_{0} \cdot \mathbf{S}_{0} \cdot \Omega_{0}\right)+4 \mathcal{D}\left(\mathbf{S}_{1} \cdot \Omega_{0}\right)\right. \\
& \left.\quad-12 \mathcal{D}\left(\mathbf{R}_{0}^{2} \cdot \mathbf{S}_{0}\right)+12 \mathcal{D}\left(\mathbf{R}_{0} \cdot \mathbf{S}_{0} \cdot \mathbf{R}_{0}\right)\right) \\
= & \frac{l^{2}}{2}\left(2 \mathcal{D}\left(\mathbf{S}_{2}\right)+8 \mathbf{S}_{0}^{3}+12 \mathcal{D}\left(\mathbf{S}_{0} \cdot \mathbf{S}_{1}\right)+4 \mathcal{D}\left(\mathbf{R}_{0}^{2} \cdot \mathbf{S}_{0}\right)-4 \mathcal{D}\left(\mathbf{R}_{0} \cdot \mathbf{S}_{0} \cdot \mathbf{R}_{0}\right)+8 \mathcal{D}\left(\mathbf{R}_{0} \cdot \mathbf{S}_{1}\right)\right),
\end{aligned}
$$

where I used equation (A.17) in the second line.

The full diagonalized shape tensor is now obtained by inserting the above results into equation (A.13). 


\section{B. Flow Properties}

In this appendix, some properties of the experimental flow are presented. In appendix B.1, the homogeneity and isotropy of the flow are studied. Appendix B.2 gives an overview of the properties of the perceived velocity gradient tensor for all datasets.

\section{B.1. Isotropy and Homogeneity}

In order to measure the isotropy and homogeneity of the flow, the mean velocity field was computed by dividing the whole measurement volume into several bins and time-averaging over the velocities of all tracer particles detected in each bin. The time average was taken over all videos in one experiment, corresponding to approximately seven hours, and is denoted by an overline.

The figures below show the absolute value (figures a, c, e) and direction (figures b, d, f) of the radial velocity, normalized by the r.m.s radial velocity at the origin, $\left.\overline{\left(u_{x}^{2}+u_{y}^{2}\right)^{1 / 2}}\right|_{O}$. Cuts at three different heights are shown $(z=20 \mathrm{~mm}, 0 \mathrm{~mm}$, and $-20 \mathrm{~mm})$ with all tracer particles within $z \pm 5 \mathrm{~mm}$ taken into account. The large measurement volume dataset with Reynolds number $R_{\lambda}=350$ was chosen representatively.

One finds that the deviations of the r.m.s. radial velocity from its value at the origin are below $20 \%$ in most of the measurement volume. Close to the borders of the measurement volume, the values are lowest. Part of this effect could be an artifact of the low statistics in this region (all bins with at least 100 detected particles are shown). In the vector plots, a directional flow towards negative $x$-values can be seen. This indicates that the windows (probably especially the flanges of the top and bottom window) disturb the rotational symmetry of the flow.

It can thus be seen that while the flow shows sufficient homogeneity, especially for the small measurement volume datasets with a measurement volume diameter of only $27 \mathrm{~mm}$, isotropy cannot be assumed. 

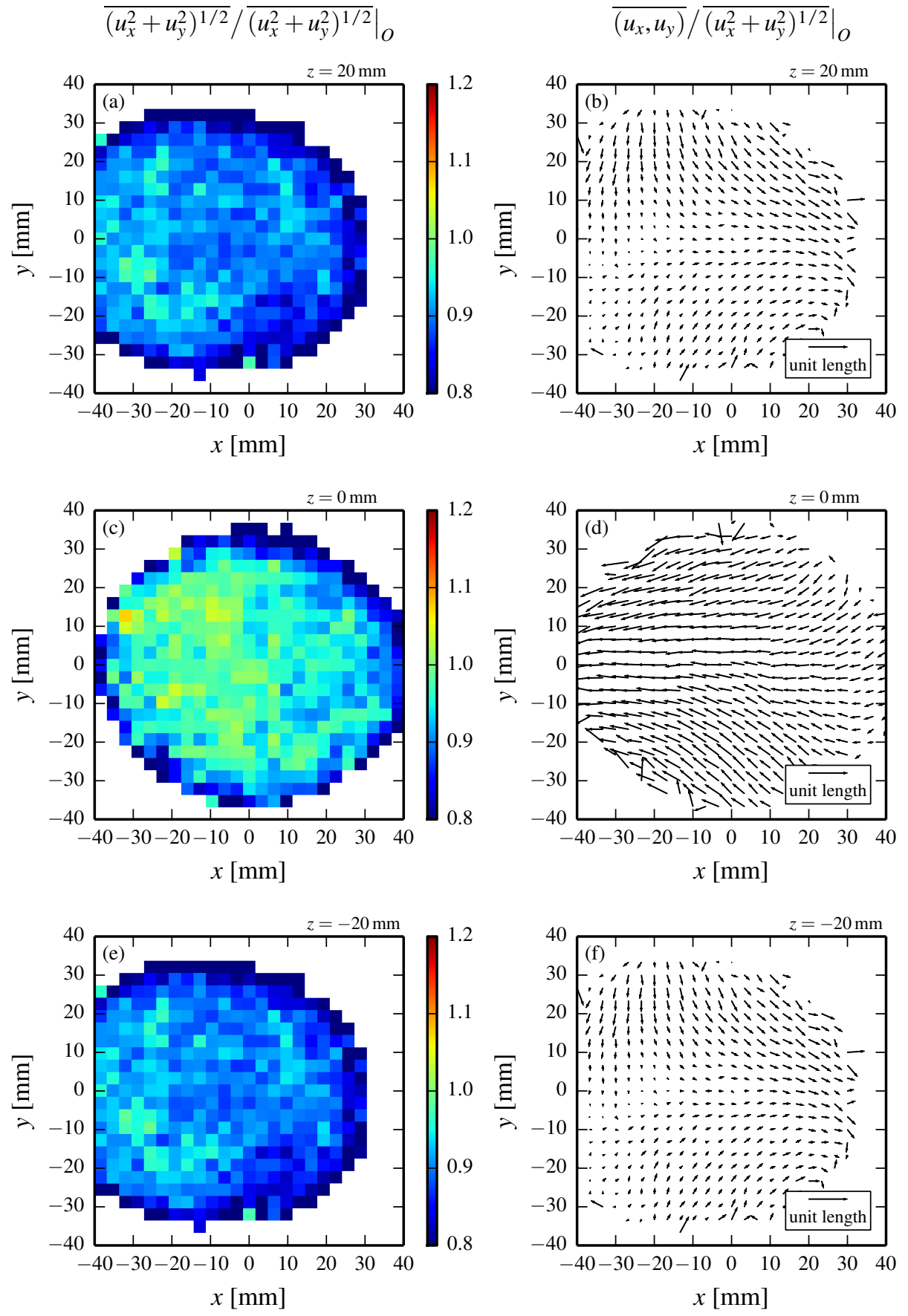

Figure B.1.: Root mean square value (left) and direction (right) of the radial velocity at three different heights, normalized by the r.m.s value at the origin. The large measurement volume dataset at $R_{\lambda}=350$ is shown. See text for more details. 


\section{B.2. Perceived Velocity Gradient Tensor}

In this appendix, several quantities derived from the perceived velocity gradient tensor are presented. Different initial separations for all datasets have been analyzed, only using the small measurement volume data and tetrahedra fulfilling the regularity condition ${ }^{1}$. The results are summarized in table 6.1 for flows without added polymers and tables 6.2 and 6.3 for flows with a polymer concentration of $5 \mathrm{ppm}$ and $10 \mathrm{ppm}$, respectively.

\begin{tabular}{|c|c|c|c|c|c|c|c|c|c|}
\hline dataset & \multicolumn{3}{|c|}{$0.5 \mathrm{~Hz} \leftrightarrow R_{\lambda}=270$} & \multicolumn{3}{|c|}{$0.9 \mathrm{~Hz} \leftrightarrow R_{\lambda}=350$} & \multicolumn{3}{|c|}{$1.1 \mathrm{~Hz} \leftrightarrow R_{\lambda}=390$} \\
\hline$R_{0}$ & $8 \mathrm{~mm}$ & $10 \mathrm{~mm}$ & $12 \mathrm{~mm}$ & $8 \mathrm{~mm}$ & $10 \mathrm{~mm}$ & $12 \mathrm{~mm}$ & $8 \mathrm{~mm}$ & $10 \mathrm{~mm}$ & $12 \mathrm{~mm}$ \\
\hline$R_{0} / \eta$ & 77 & 96 & 115 & 121 & 152 & 182 & 138 & 172 & 207 \\
\hline$\left\langle s_{1}\right\rangle t_{0}$ & 2.27 & 2.26 & 2.30 & 2.17 & 2.21 & 2.23 & 2.21 & 2.25 & 2.28 \\
\hline$\left\langle s_{2}\right\rangle t_{0}$ & 0.22 & 0.22 & 0.24 & 0.21 & 0.22 & 0.23 & 0.21 & 0.22 & 0.23 \\
\hline$\left\langle s_{3}\right\rangle t_{0}$ & -2.44 & -2.49 & -2.54 & -2.36 & -2.42 & -2.45 & -2.42 & -2.46 & -2.49 \\
\hline$\left\langle\operatorname{tr}\left(\mathbf{S}_{0}\right)\right\rangle t_{0}$ & 0.05 & 0.00 & -0.01 & 0.01 & 0.01 & 0.02 & 0.01 & 0.02 & 0.01 \\
\hline$\left\langle s_{1}^{2}\right\rangle t_{0}^{2}$ & 6.41 & 6.37 & 6.56 & 5.90 & 6.11 & 6.22 & 6.16 & 6.33 & 6.43 \\
\hline$\left\langle s_{2}^{2}\right\rangle t_{0}^{2}$ & 0.94 & 0.93 & 0.97 & 0.87 & 0.89 & 0.92 & 0.90 & 0.93 & 0.95 \\
\hline$\left\langle s_{3}^{2}\right\rangle t_{0}^{2}$ & 7.85 & 8.10 & 8.39 & 7.40 & 7.72 & 7.86 & 7.72 & 7.92 & 8.09 \\
\hline$\left\langle\operatorname{tr}\left(\mathbf{S}_{0}^{2}\right)\right\rangle t_{0}^{2}$ & 15.20 & 15.40 & 15.92 & 14.16 & 14.72 & 14.99 & 14.79 & 15.19 & 15.46 \\
\hline$\left\langle\operatorname{tr}\left(\mathbf{S}_{0}\right)^{2}\right\rangle t_{0}^{2}$ & 4.26 & 4.28 & 4.39 & 4.05 & 4.13 & 4.21 & 4.18 & 4.30 & 4.36 \\
\hline$\left\langle s_{1}^{3}\right\rangle t_{0}^{3}$ & 21.54 & 21.12 & 21.98 & 19.08 & 19.98 & 20.42 & 20.36 & 21.03 & 21.31 \\
\hline$\left\langle s_{2}^{3}\right\rangle t_{0}^{3}$ & 0.58 & 0.63 & 0.64 & 0.52 & 0.56 & 0.61 & 0.54 & 0.59 & 0.59 \\
\hline$\left\langle s_{3}^{3}\right\rangle t_{0}^{3}$ & -30.66 & -31.72 & -33.08 & -28.21 & -29.59 & -30.20 & -29.87 & -30.69 & -31.44 \\
\hline$\left\langle\operatorname{tr}\left(\mathbf{S}_{0}^{3}\right)\right\rangle t_{0}^{3}$ & -8.53 & -9.98 & -10.46 & -8.61 & -9.05 & -9.17 & -8.97 & -9.08 & -9.53 \\
\hline$\left\langle\operatorname{tr}\left(\mathbf{S}_{0}\right) \operatorname{tr}\left(\mathbf{S}_{0}^{2}\right)\right\rangle t_{0}^{3}$ & -1.78 & -2.53 & -2.68 & -2.30 & -2.35 & -2.15 & -2.37 & -2.17 & -2.44 \\
\hline$\left\langle\left(\mathbf{S}_{0} \cdot \mathbf{S}_{1}\right)_{11}\right\rangle t_{0}^{3}$ & -7.62 & -6.50 & -6.99 & -6.14 & -6.36 & -6.36 & -7.43 & -7.38 & -7.77 \\
\hline$\left\langle\left(\mathbf{S}_{0} \cdot \mathbf{S}_{1}\right)_{22}\right\rangle t_{0}^{3}$ & -0.05 & 0.04 & 0.08 & 0.12 & 0.05 & 0.02 & 0.04 & 0.02 & 0.06 \\
\hline$\left\langle\left(\mathbf{S}_{0} \cdot \mathbf{S}_{1}\right)_{33}\right\rangle t_{0}^{3}$ & 9.58 & 9.30 & 10.07 & 9.32 & 9.36 & 8.95 & 9.87 & 10.30 & 10.53 \\
\hline$\left\langle\operatorname{tr}\left(\mathbf{S}_{0} \cdot \mathbf{S}_{1}\right)\right\rangle t_{0}^{3}$ & 1.91 & 2.84 & 3.16 & 3.31 & 3.05 & 2.61 & 2.49 & 2.94 & 2.82 \\
\hline$\left\langle\operatorname{tr}\left(\mathbf{S}_{0}\right) \operatorname{tr}\left(\mathbf{S}_{1}\right)\right\rangle t_{0}^{3}$ & 0.07 & 0.81 & 0.69 & 1.42 & 0.70 & 0.27 & 0.64 & 0.59 & 0.56 \\
\hline$\left\langle\operatorname{tr}\left(\Omega_{0}^{2}\right)\right\rangle t_{0}^{2}$ & -9.98 & -10.49 & -10.59 & -9.62 & -9.96 & -10.05 & -10.16 & -10.50 & -10.58 \\
\hline$\left\langle\operatorname{tr}\left(\mathbf{S}_{0} \cdot \Omega_{0}^{2}\right)\right\rangle t_{0}^{3}$ & 1.38 & 1.82 & 2.04 & 1.57 & 1.77 & 1.80 & 1.62 & 1.85 & 1.93 \\
\hline$\left\langle\operatorname{tr}\left(\mathbf{S}_{0}\right) \operatorname{tr}\left(\Omega_{0}^{2}\right)\right\rangle t_{0}^{3}$ & -1.16 & -0.64 & -0.37 & -0.64 & -0.32 & -0.48 & -0.52 & -0.55 & -0.52 \\
\hline$\left\langle\operatorname{tr}\left(\Omega_{0} \cdot \Omega_{1}\right)\right\rangle t_{0}^{3}$ & -0.98 & -1.18 & -0.07 & -0.47 & -1.18 & -0.85 & -0.46 & -1.37 & -1.45 \\
\hline
\end{tabular}

Table 6.1.: Quantities derived from the perceived velocity gradient tensor for datasets with no added polymers $(0 \mathrm{ppm})$. For time derivatives such as $\mathbf{S}_{1}$ and $\Omega_{1}$, a Gaussian convolution filter was used with a filter length of $3 \tau_{\eta}$ and a width of $\tau_{\eta}$.

\footnotetext{
${ }^{1}$ The dataset with $R_{\lambda}=690$ was omitted since it only consists of a large measurement volume dataset.
} 


\begin{tabular}{|c|c|c|c|c|c|c|c|c|}
\hline dataset & \multicolumn{4}{|c|}{$0.9 \mathrm{~Hz} \leftrightarrow R_{\lambda}=350$} & \multicolumn{4}{|c|}{$1.1 \mathrm{~Hz} \leftrightarrow R_{\lambda}=390$} \\
\hline$R_{0}$ & $8 \mathrm{~mm}^{\diamond}$ & $10 \mathrm{~mm}$ & $12 \mathrm{~mm}$ & $14 \mathrm{~mm}$ & $8 \mathrm{~mm}^{\diamond}$ & $10 \mathrm{~mm}$ & $12 \mathrm{~mm}$ & $14 \mathrm{~mm}$ \\
\hline$R_{0} / r_{\varepsilon}$ & $1.1^{\diamond}$ & 1.3 & 1.6 & 1.8 & $1.0^{\diamond}$ & 1.2 & 1.5 & 1.7 \\
\hline$\left\langle s_{1}\right\rangle t_{0}$ & 1.77 & 1.89 & 1.98 & 2.03 & 1.87 & 1.99 & 2.08 & 2.13 \\
\hline$\left\langle s_{2}\right\rangle t_{0}$ & 0.26 & 0.29 & 0.30 & 0.31 & 0.28 & 0.29 & 0.31 & 0.32 \\
\hline$\left\langle s_{3}\right\rangle t_{0}$ & -2.05 & -2.16 & -2.24 & -2.29 & -2.13 & -2.25 & -2.34 & -2.37 \\
\hline$\left\langle\operatorname{tr}\left(\mathbf{S}_{0}\right)\right\rangle t_{0}$ & -0.03 & 0.03 & 0.03 & 0.05 & 0.02 & 0.02 & 0.04 & 0.07 \\
\hline$\left\langle s_{1}^{2}\right\rangle t_{0}^{2}$ & 3.85 & 4.37 & 4.76 & 5.01 & 4.32 & 4.85 & 5.26 & 5.51 \\
\hline$\left\langle s_{2}^{2}\right\rangle t_{0}^{2}$ & 0.51 & 0.60 & 0.65 & 0.70 & 0.58 & 0.67 & 0.73 & 0.77 \\
\hline$\left\langle s_{3}^{2}\right\rangle t_{0}^{2}$ & 5.28 & 5.87 & 6.35 & 6.61 & 5.74 & 6.41 & 6.90 & 7.11 \\
\hline$\left\langle\operatorname{tr}\left(\mathbf{S}_{0}^{2}\right)\right\rangle t_{0}^{2}$ & 9.64 & 10.83 & 11.76 & 12.33 & 10.64 & 11.92 & 12.89 & 13.38 \\
\hline$\left\langle\operatorname{tr}\left(\mathbf{S}_{0}\right)^{2}\right\rangle t_{0}^{2}$ & 1.46 & 1.82 & 2.12 & 2.35 & 1.74 & 2.13 & 2.44 & 2.74 \\
\hline$\left\langle s_{1}^{3}\right\rangle t_{0}^{3}$ & 9.97 & 11.94 & 13.45 & 14.45 & 11.88 & 14.01 & 15.73 & 16.75 \\
\hline$\left\langle s_{2}^{3}\right\rangle t_{0}^{3}$ & 0.38 & 0.48 & 0.54 & 0.59 & 0.46 & 0.56 & 0.60 & 0.66 \\
\hline$\left\langle s_{3}^{3}\right\rangle t_{0}^{3}$ & -16.27 & -19.00 & -21.34 & -22.63 & -18.62 & -21.73 & -24.12 & -25.18 \\
\hline$\left\langle\operatorname{tr}\left(\mathbf{S}_{0}^{3}\right)\right\rangle t_{0}^{3}$ & -5.92 & -6.57 & -7.36 & -7.59 & -6.28 & -7.17 & -7.79 & -7.76 \\
\hline$\left\langle\operatorname{tr}\left(\mathbf{S}_{0}\right) \operatorname{tr}\left(\mathbf{S}_{0}^{2}\right)\right\rangle t_{0}^{3}$ & -0.53 & -0.17 & -0.37 & -0.19 & -0.26 & -0.24 & -0.21 & 0.13 \\
\hline$\left\langle\left(\mathbf{S}_{0} \cdot \mathbf{S}_{1}\right)_{11}\right\rangle t_{0}^{3}$ & -2.33 & -2.62 & -2.89 & -3.17 & -2.72 & -3.29 & -3.82 & -3.74 \\
\hline$\left\langle\left(\mathbf{S}_{0} \cdot \mathbf{S}_{1}\right)_{22}\right\rangle t_{0}^{3}$ & 0.06 & 0.14 & 0.18 & 0.22 & 0.15 & 0.25 & 0.26 & 0.21 \\
\hline$\left\langle\left(\mathbf{S}_{0} \cdot \mathbf{S}_{1}\right)_{33}\right\rangle t_{0}^{3}$ & 3.49 & 3.81 & 3.98 & 4.58 & 3.45 & 4.40 & 5.55 & 5.71 \\
\hline$\left\langle\operatorname{tr}\left(\mathbf{S}_{0} \cdot \mathbf{S}_{1}\right)\right\rangle t_{0}^{3}$ & 1.23 & 1.33 & 1.28 & 1.62 & 0.88 & 1.35 & 1.99 & 2.18 \\
\hline$\left\langle\operatorname{tr}\left(\mathbf{S}_{0}\right) \operatorname{tr}\left(\mathbf{S}_{1}\right)\right\rangle t_{0}^{3}$ & -0.29 & -0.49 & -0.30 & -0.39 & -0.51 & -0.23 & -0.14 & 0.20 \\
\hline$\left\langle\operatorname{tr}\left(\Omega_{0}^{2}\right)\right\rangle t_{0}^{2}$ & -6.86 & -7.71 & -8.22 & -8.56 & -8.01 & -8.89 & -9.46 & -9.70 \\
\hline$\left\langle\operatorname{tr}\left(\mathbf{S}_{0} \cdot \Omega_{0}^{2}\right)\right\rangle t_{0}^{3}$ & 1.65 & 1.74 & 1.92 & 1.90 & 1.80 & 2.02 & 2.15 & 2.11 \\
\hline$\left\langle\operatorname{tr}\left(\mathbf{S}_{0}\right) \operatorname{tr}\left(\Omega_{0}^{2}\right)\right\rangle t_{0}^{3}$ & 0.19 & -0.43 & -0.43 & -0.86 & -0.20 & -0.43 & -0.58 & -0.91 \\
\hline$\left\langle\operatorname{tr}\left(\Omega_{0} \cdot \Omega_{1}\right)\right\rangle t_{0}^{3}$ & -1.03 & -0.97 & -0.82 & -0.67 & -0.96 & -1.15 & -1.43 & -0.89 \\
\hline
\end{tabular}

Table 6.2.: Quantities derived from the perceived velocity gradient tensor for datasets with a polymer concentration of $5 \mathrm{ppm}$. For time derivatives such as $\mathbf{S}_{1}$ and $\Omega_{1}$, a Gaussian convolution filter was used with a filter length of $3 \tau_{\eta}$ and a width of $\tau_{\eta}$, with $\tau_{\eta}$ taken from the corresponding dataset without polymers. Initial separations marked with a diamond $(\diamond)$ lie outside the plateau range of the energy transfer rate of the respective dataset. The used value of $t_{0}$, however, is always based on the plateau value. 


\begin{tabular}{|c|c|c|c|c|c|c|c|c|}
\hline \multirow{2}{*}{$\begin{array}{l}\text { dataset } \\
R_{0}\end{array}$} & \multicolumn{4}{|c|}{$0.9 \mathrm{~Hz} \leftrightarrow R_{\lambda}=350$} & \multicolumn{4}{|c|}{$1.1 \mathrm{~Hz} \leftrightarrow R_{\lambda}=390$} \\
\hline & $8 \mathrm{~mm}^{\diamond}$ & $10 \mathrm{~mm}^{\diamond}$ & $12 \mathrm{~mm}$ & $14 \mathrm{~mm}$ & $8 \mathrm{~mm}^{\diamond}$ & $10 \mathrm{~mm} \diamond$ & $12 \mathrm{~mm}$ & $14 \mathrm{~mm}$ \\
\hline$R_{0} / r_{\varepsilon}$ & $0.8^{\diamond}$ & $1^{\diamond}$ & 1.2 & 1.4 & $0.7^{\diamond}$ & $0.9^{\diamond}$ & 1.1 & 1.3 \\
\hline$\left\langle s_{1}\right\rangle t_{0}$ & 1.70 & 1.85 & 1.98 & 2.07 & 1.67 & 1.81 & 1.93 & 2.03 \\
\hline$\left\langle s_{2}\right\rangle t_{0}$ & 0.27 & 0.30 & 0.33 & 0.35 & 0.28 & 0.30 & 0.32 & 0.34 \\
\hline$\left\langle s_{3}\right\rangle t_{0}$ & -1.97 & -2.15 & -2.29 & -2.40 & -1.93 & -2.10 & -2.23 & -2.33 \\
\hline$\left\langle\operatorname{tr}\left(\mathbf{S}_{0}\right)\right\rangle t_{0}$ & 0.01 & 0.00 & 0.02 & 0.02 & 0.01 & 0.01 & 0.02 & 0.03 \\
\hline$\left\langle s_{1}^{2}\right\rangle t_{0}^{2}$ & 3.60 & 4.22 & 4.78 & 5.21 & 3.45 & 4.04 & 4.56 & 5.00 \\
\hline$\left\langle s_{2}^{2}\right\rangle t_{0}^{2}$ & 0.44 & 0.53 & 0.62 & 0.69 & 0.44 & 0.53 & 0.61 & 0.68 \\
\hline$\left\langle s_{3}^{2}\right\rangle t_{0}^{2}$ & 4.84 & 5.78 & 6.53 & 7.13 & 4.71 & 5.53 & 6.19 & 6.77 \\
\hline$\left\langle\operatorname{tr}\left(\mathbf{S}_{0}^{2}\right)\right\rangle t_{0}^{2}$ & 8.88 & 10.54 & 11.92 & 13.03 & 8.60 & 10.10 & 11.35 & 12.45 \\
\hline$\left\langle\operatorname{tr}\left(\mathbf{S}_{0}\right)^{2}\right\rangle t_{0}^{2}$ & 1.12 & 1.35 & 1.58 & 1.98 & 1.12 & 1.14 & 1.71 & 1.88 \\
\hline$\left\langle s_{1}^{3}\right\rangle t_{0}^{3}$ & 9.11 & 11.45 & 13.31 & 15.36 & 8.60 & 10.76 & 12.77 & 14.56 \\
\hline$\left\langle s_{2}^{3}\right\rangle t_{0}^{3}$ & 0.34 & 0.45 & 0.55 & 0.64 & 0.35 & 0.45 & 0.53 & 0.61 \\
\hline$\left\langle s_{3}^{3}\right\rangle t_{0}^{3}$ & -14.20 & -18.40 & -21.97 & -24.92 & -13.73 & -17.33 & -20.35 & -23.16 \\
\hline$\left\langle\operatorname{tr}\left(\mathbf{S}_{0}^{3}\right)\right\rangle t_{0}^{3}$ & -4.74 & -6.51 & -7.80 & -8.92 & -4.78 & -6.12 & -7.05 & -7.99 \\
\hline$\left\langle\operatorname{tr}\left(\mathbf{S}_{0}\right) \operatorname{tr}\left(\mathbf{S}_{0}^{2}\right)\right\rangle t_{0}^{3}$ & 0.28 & 0.10 & 0.16 & -0.04 & 0.15 & 0.10 & 0.14 & 0.09 \\
\hline$\left\langle\left(\mathbf{S}_{0} \cdot \mathbf{S}_{1}\right)_{11}\right\rangle t_{0}^{3}$ & -1.82 & -2.30 & -2.81 & -2.94 & -1.95 & -2.30 & -2.82 & -2.90 \\
\hline$\left\langle\left(\mathbf{S}_{0} \cdot \mathbf{S}_{1}\right)_{22}\right\rangle t_{0}^{3}$ & 0.11 & 0.14 & 0.16 & 0.23 & 0.10 & 0.15 & 0.19 & 0.20 \\
\hline$\left\langle\left(\mathbf{S}_{0} \cdot \mathbf{S}_{1}\right)_{33}\right\rangle t_{0}^{3}$ & 2.35 & 2.93 & 3.24 & 3.84 & 2.21 & 2.95 & 3.60 & 3.78 \\
\hline$\left\langle\operatorname{tr}\left(\mathbf{S}_{0} \cdot \mathbf{S}_{1}\right)\right\rangle t_{0}^{3}$ & 0.65 & 0.78 & 0.59 & 1.13 & 0.35 & 0.80 & 0.97 & 1.08 \\
\hline$\left\langle\operatorname{tr}\left(\mathbf{S}_{0}\right) \operatorname{tr}\left(\mathbf{S}_{1}\right)\right\rangle t_{0}^{3}$ & -0.55 & -0.55 & -0.67 & -0.54 & -0.54 & -0.48 & -0.54 & -0.54 \\
\hline$t_{0}^{2}\left\langle\operatorname{tr}\left(\Omega_{0}^{2}\right)\right\rangle$ & -6.81 & -7.76 & -8.45 & -9.48 & -6.69 & -7.76 & -8.68 & -9.23 \\
\hline$t_{0}^{3}\left\langle\operatorname{tr}\left(\mathbf{S}_{0} \cdot \Omega_{0}^{2}\right)\right\rangle$ & 1.54 & 2.01 & 2.30 & 2.76 & 1.48 & 1.85 & 2.15 & 2.42 \\
\hline$t_{0}^{3}\left\langle\operatorname{tr}\left(\mathbf{S}_{0}\right) \operatorname{tr}\left(\Omega_{0}^{2}\right)\right\rangle$ & -0.10 & -0.07 & -0.07 & -0.24 & -0.10 & -0.12 & -0.25 & -0.30 \\
\hline$t_{0}^{3}\left\langle\operatorname{tr}\left(\Omega_{0} \cdot \Omega_{1}\right)\right\rangle$ & -0.88 & -1.27 & -1.13 & -1.55 & -0.76 & -1.15 & -1.21 & -1.33 \\
\hline
\end{tabular}

Table 6.3.: Quantities derived from the perceived velocity gradient tensor for datasets with a polymer concentration of $10 \mathrm{ppm}$. For time derivatives such as $\mathbf{S}_{1}$ and $\Omega_{1}$, a Gaussian convolution filter was used with a filter length of $3 \tau_{\eta}$ and a width of $\tau_{\eta}$, with $\tau_{\eta}$ taken from the corresponding dataset without polymers. Initial separations marked with a diamond $(\diamond)$ lie outside the plateau range of the energy transfer rate of the respective dataset. The used value of $t_{0}$, however, is always based on the plateau value. 


\section{Data Formats}

In this appendix, the used binary data formats are presented. Each line represents a variable that is written to the binary file in the order given below if not indicated otherwise by the comments. The first column gives the $\mathrm{C}++$ data type and length of a variable, the second column gives its name. Double slashes "//" indicate comments. As an example,

unsigned int [5] test; // numbers one to five

would mean that the array "test" is made of five positive integers which contain the numbers from one to five.

\section{C.1. Göttingen Movie (.gmv)}

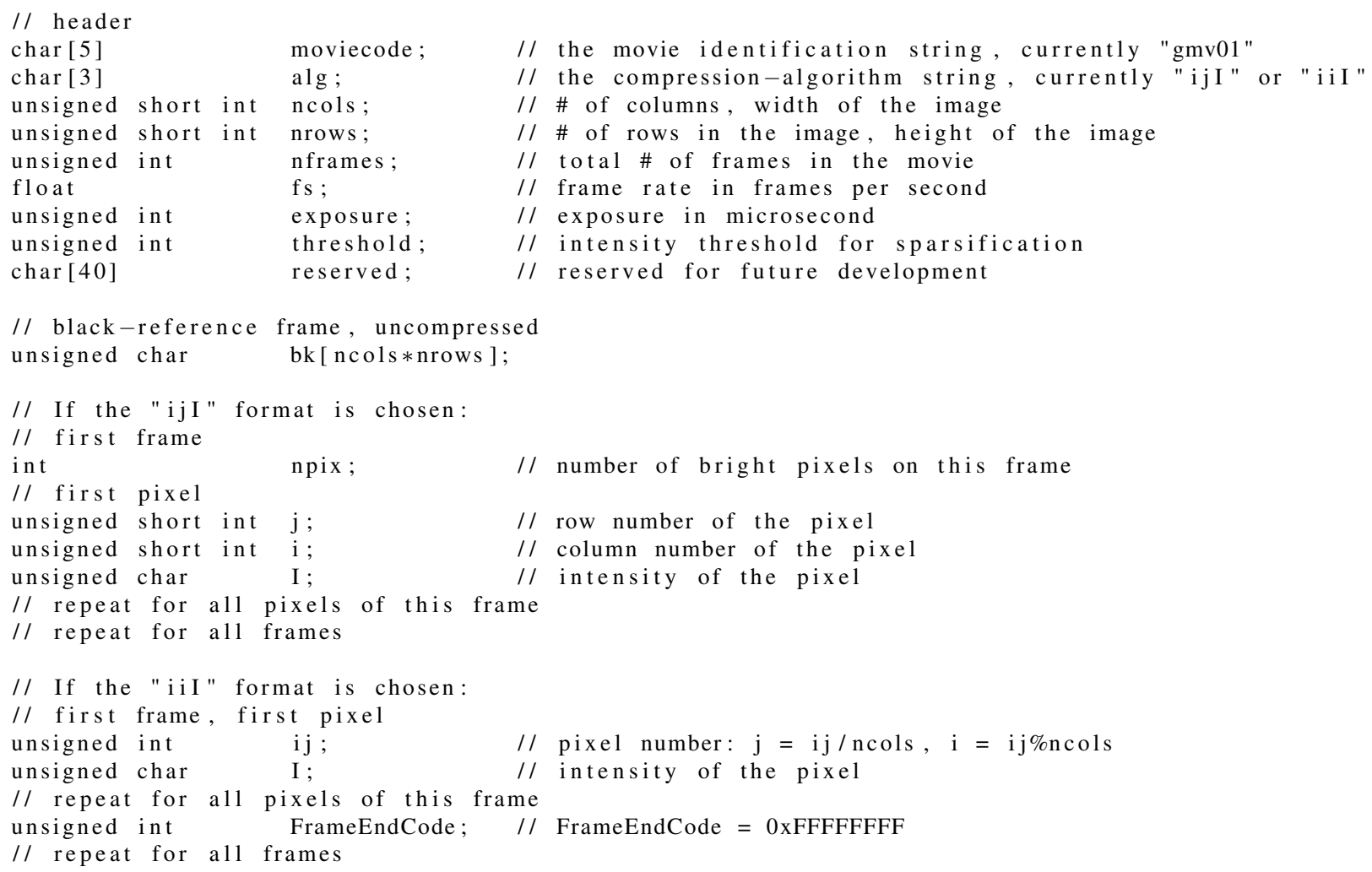

\section{C.2. Trajectory Data Format}

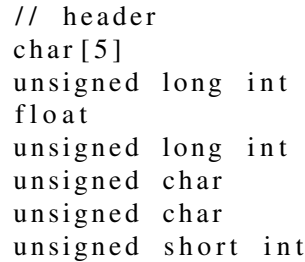

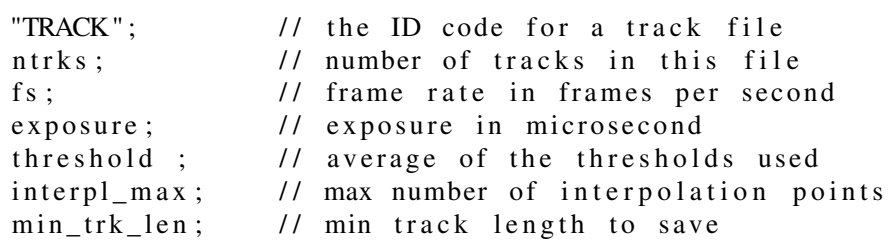




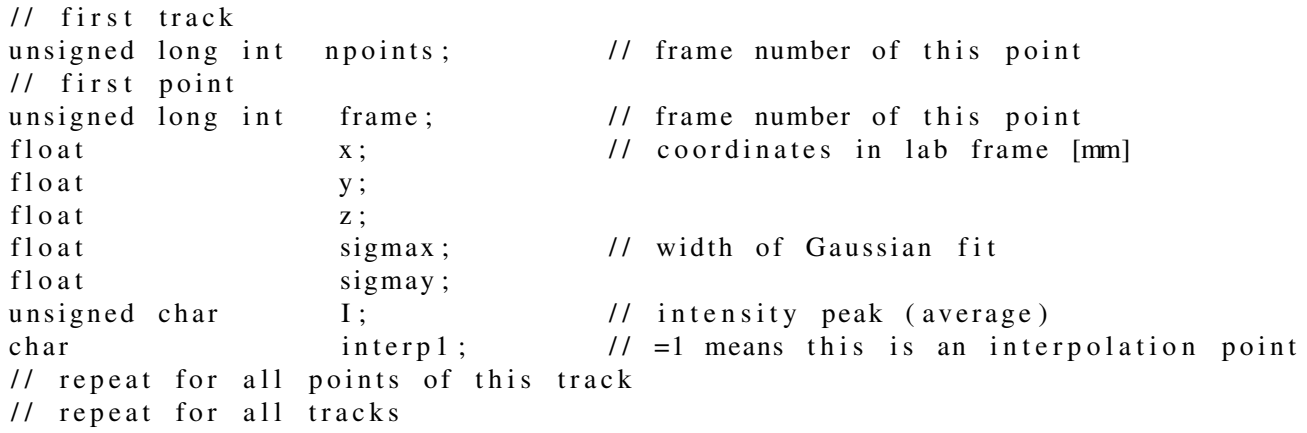

\section{C.3. Velocity-Acceleration Data Format}

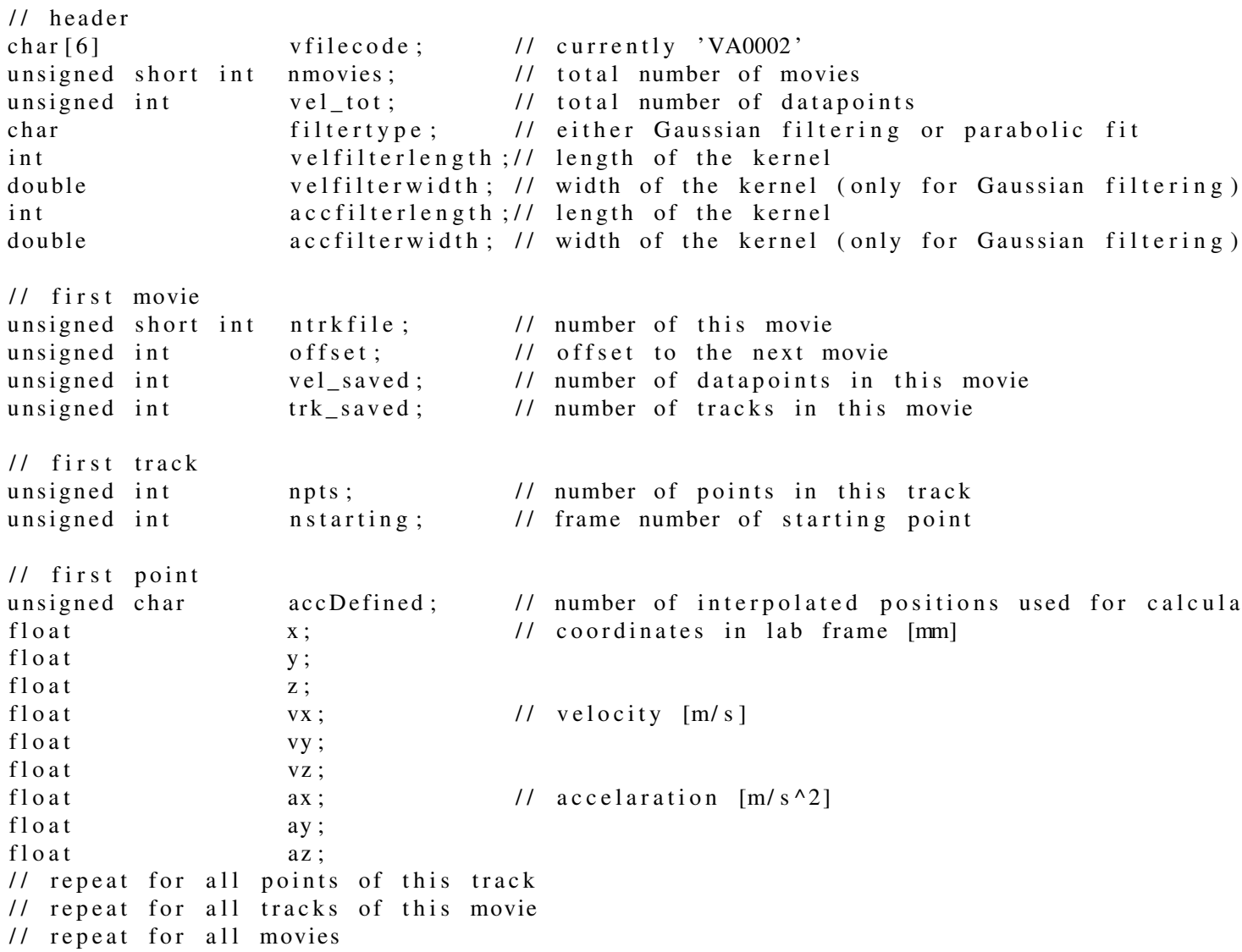

\section{C.4. Tetrahedra Data Format}

$\begin{array}{lll}\text { // header } & \text { filecode; } & / / \text { a string "tetrads" as to identify the file } \\ \text { char[7] } & \text { nmov; } & / / \text { movie number } \\ \text { unsigned short int } & \text { ro; } & / / \text { tetrad edge length in mm } \\ \text { double } & & \end{array}$




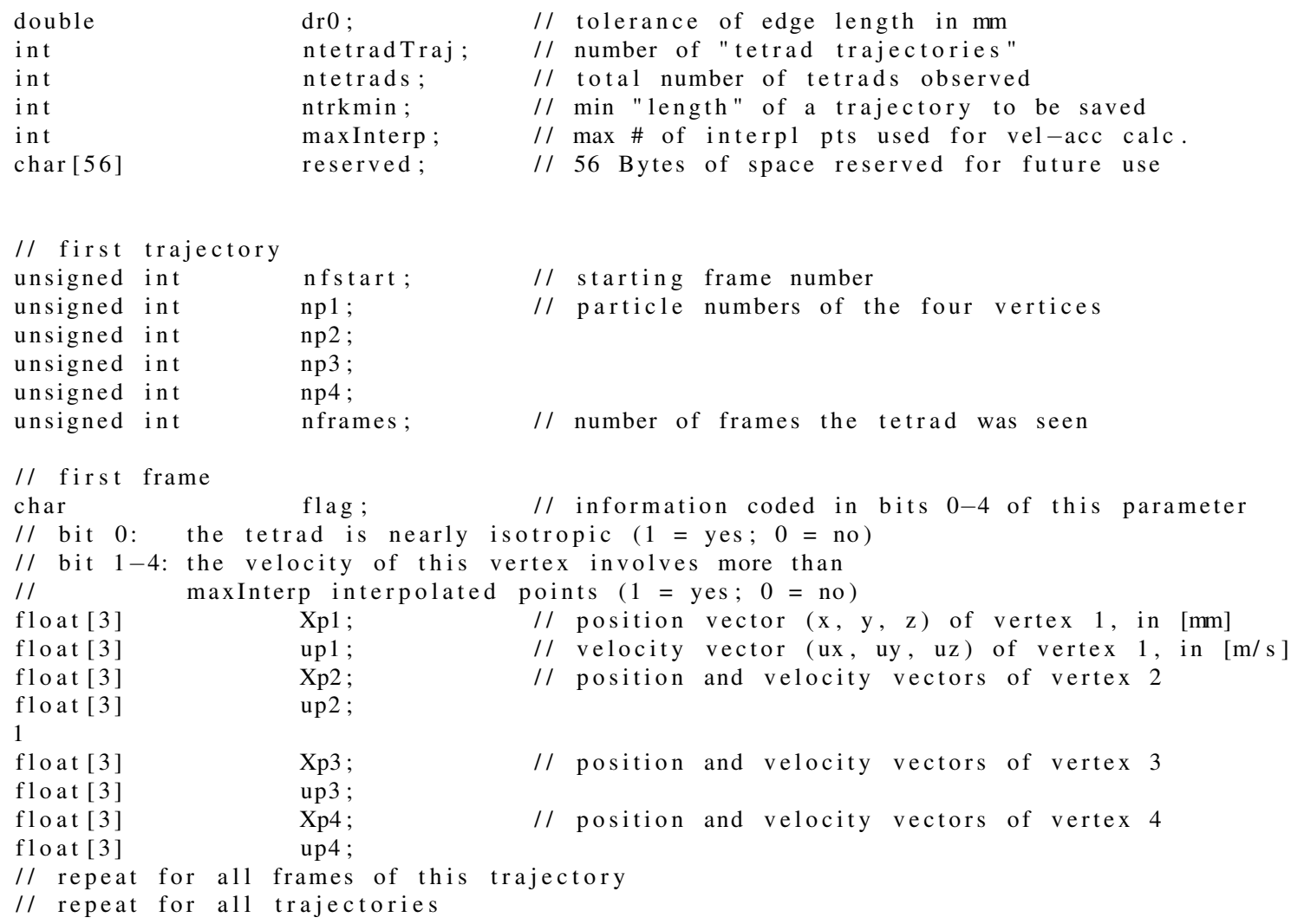




\section{Bibliography}

ADRIAN, R. 1991 Particle-imaging techniques for experimental fluid mechanics. Annu. Rev. Fluid Mech. 23, 261-304.

Argyris, J., FAust, G., HaAse, M. \& Friedrich, R. 2010 Die Erforschung des Chaos. Springer.

Avila, K., Moxey, D., De Lozar, A., Avila, M., Barkley, D. \& Hof, B. 2011 The onset of turbulence in pipe flow. Science 333, 192-196.

Bailey, F. E. \& Koleske, J. V. 1976 Poly(ethylene oxide). Academic Press, New York.

BATCHELOR, G. 1950 The application of the similarity theory of turbulence to atmospheric diffusion. Q. J. Roy. Meteor. Soc. 76, 133-146.

BAtchelor, G. 1952 Diffusion in a field of homogeneous turbulence. Proc. Cambridge Philos. Soc. 48, 345-362.

BERG, J., LÜTHI, B., MANN, J. \& OTT, S. 2006 Backwards and forwards relative dispersion in turbulent flow: An experimental investigation. Phys. Rev. E 74, 016304.

Berti, S., Bistagnino, A., Boffetta, G., Celani, A. \& Musacchio, S. 2006 Small-scale statistics of viscoelastic turbulence. Europhys. Lett. 76, 63-69.

BETCHOV, R. 1956 An inequality concerning the production of vorticity in isotropic turbulence. J. Fluid Mech. 1, 497-504.

Biferale, L., Boffetta, G., Celani, A., Devenish, J. \& Lanotte, A. 2005 Multiparticle dispersion in fully developed turbulence. Phys. Fluids 17, 111701.

Bodenschatz, E., Malinowski, S. P., Shaw, R. A. \& Stratmann, F. 2010 Can we understand clouds without turbulence? Science 327, 970-971.

BoffettA, G. \& Ecke, R. E. 2012 Two-dimensional turbulence. Annu. Rev. Fluid Mech. 44, 427-451. 
Bonn, D., Couder, Y., VAn Dam, P. H. J. \& DouAdy, S. 1993 From small scales to large scales in three-dimensional turbulence: The effect of diluted polymers. Phys. Rev. E 47, 28-31.

Bourgoin, M., Ouellette, N. T., Xu, H., Berg, J. \& Bodenschatz, E. 2006 The role of pair dispersion in turbulent flow. Science 311, 835-838.

Bragg, A. D., Ireland, P. J. \& Collins, L. R. 2014 Forward and backward in time dispersion of fluid and inertial patricles in isotropic turbulence. [arXiv:1403.5502v1].

Celani, A., Cencini, M., Mazzino, A. \& Vergassola, M. 2004 Active and passive fields face to face. New J. Phys. 6, 72.

Chertkov, M., Pumir, A. \& Shraiman, B. 1999 Lagrangian tetrad dynamics and the phenomenology of turbulence. Phys. Fluids 11, 2394-2410.

ChIU, W. C. \& RIB, L. N. 1956 The rate of dissipation of energy and the energy spectrum in a low-speed turbulent jet. Trans. Am. Geophys. Union 37, 13-26.

Corrsin, S. 1952 Heat transfer in isotropic turbulence. J. Appl. Phys. 23, 113-118.

Cowen, E. A. \& Monismith, S. G. 1997 A hybrid digital particle tracking velocimetry technique. Exp. Fluids 22, 199-211.

Crawford, A. M., Mordant, N., Xu, H. \& Bodenschatz, E. 2008 Fluid acceleration in the bulk of turbulent dilute polymer solutions. New J. Phys. 10, 123015.

Davidson, P. A., Kaneda, Y., Moffatt, K. \& Sreenivasan, K. R. 2011 A voyage through turbulence. Cambridge University Press.

De Gennes, P. G. 1986 Towards a scaling theory of drag reduction. Physica A 140, 9-25.

Dimotakis, P. E. 2005 Turbulent mixing. Annu. Rev. Fluid Mech. 37, 329-356.

DURBIN, P. 1980 A stochastic model of two-particle dispersion and concentration fluctuations in homogeneous turbulence. J. Fluid Mech. 100, 279-302.

Falkovich, G., Gawedzki, K. \& Vergassola, M. 2001 Particles and fields in fluid turbulence. Rev. Mod. Phys. 73, 913-975.

Falkovich, G., Xu, H., Pumir, A., Bodenschatz, E., Biferale, L., Boffetta, G., LAnotte, A. S. \& Toschi, F. 2012 On lagrangian single-particle statistics. Phys. Fluids 24, 055102.

FRISCH, U. 1995 Turbulence. Cambridge University Press.

Frisch, U., Mazzino, A., Noullez, A. \& Vergassola, M. 1999 Lagrangian method for multiple correlations in passive scalar advection. Phys. Fluids 11, 2178-2186.

Frishman, A. 2013 Private Communication. 
HACKL, J. F., YeunG, P. K. \& SAWfORD, B. L. 2011 Multi-particle and tetrad statistics in numerical simulations of turbulent relative dispersion. Phys. Fluids 23, 065103.

Heller, J. P. 1960 An unmixing demonstration. Am. J. Phys. 28, 348-353.

Hoyt, J. W. 1972 A Freeman Scholar Lecture: The effect of dditives on fluid friction. J. Basic Eng. 94, 258-285.

Jucha, J., Xu, H., Pumir, A. \& Bodenschatz, E. 2014 Time-reversal-symmetry breaking in turbulence. Phys. Rev. Lett. 113, 054501.

KING, L. V. 1914 On the convection of heat from small cylinders in a stream of uid. determination of convective constants of small platinum wires with application to hot-wire anemometry. Phil. Trans. Roy. Soc. Lond. A 214, 373-432.

Kolmogorov, A. N. 1941a The local structure of turbulence in incompressible viscous fluid for very large reynolds numbers. Dokl. Akad. Nauk SSSR 30, 299-303.

Kolmogorov, A. N. $1941 b$ Dissipation of energy in locally isotropic turbulence. Dokl. Akad. Nauk SSSR 32, 16-18.

Kraichnan, R. 1961 The closure problem of turbulence theory. Tech. Rep.. DTIC Document.

Kraichnan, R. 1967 Inertial ranges in two-dimensional turbulence. Tech. Rep.. DTIC Document.

Li, Y., Perlman, E., Wan, M., Yang, Y., Meneveau, C., Burns, R., Chen, S., SZALAY, A. \& EYINK, G. 2008 A public turbulence database cluster and applications to study lagrangian evolution of velocity increments in turbulence. J. Turbul. 9, 31.

Liberzon, A., Guala, M., Kinzelbach, W. \& Tsinober, A. 2006 On turbulent kinetic energy production and dissipation in dilute polymer solutions. Phys. Fluids 18, 125101.

Liberzon, A., Guala, M., LÜthi, B., Kinzelbach, W. \& Tsinober, A. 2005 Turbulence in dilute polymer solutions. Phys. Fluids 17, 031707.

LorenZ, E. N. 1963 Deterministic nonperiodic flow. J. Atmos. Sci. 20, 130-141.

Lumley, J. 1969 Drag reduction by additives. Annu. Rev. Fluid Mech. 1, 367-384.

Lumley, J. L. \& Yaglom, A. M. 2001 A century of turbulence. Flow Turbul. Combust. 66, 241-286.

Lundgren, T. 1981 Turbulent pair dispersion and scalar diffusion. J. Fluid Mech. 111, 27-57.

LÜthi, B., OTt, S., BERG, J. \& MANN, J. 2007 Lagrangian multi-particle statistics. $J$. Turbul. 8, 45. 
MaAs, H. G., Gruen, A. \& Papantoniou, D. 1993 Particle tracking velocimery in three-dimensional flows - part1. photogrammetric determination of particle coordinates. Exp. Fluids 15, 133-146.

Malik, N. A., Dracos, T. \& Papantoniou, D. 1993 Particle tracking velocimetry in three-dimensional flows - part 2. particle tracking. Exp. Fluids 15, 279-294.

MAnn, J., OtT, S. \& Andersen, J. S. 1999 Experimental study of relative, turbulent diffusion. Tech. Rep. (Risø-R-1036(EN)).

Monin, A. S. \& Yaglom, A. M. 2007 Statistical Fluid Mechanics - Mechanics of Turbulence, , vol. 1 and 2. Dover Publications.

Mordant, N., Crawford, A. M. \& Bodenschatz, E. 2004 Experimental lagrangian acceleration probability density function mesurement. Physica D 193, 245-251.

Naveira Garabato, A. C., Polzin, K. L., King, B. A., Heywood, K. J. \& Visbeck, M. 2004 Widespread intense turbulent mixing in the southern ocean. Science 303, 210213.

NAVIER, C. L. M. 1827 Sur les lois du mouvement des fluids. Comptes Rendus des Seances de l'Academie des Sciences 6, 389-440.

Nore, C., Tuckerman, L. S., Daube, O. \& Xin, S. 2003 The 1:2 mode interaction in exactly counter-rotating von Kármán swirling flow. J. Fluid Mech. 477, 51-88.

OBukHov, A. 1941 Spectral energy distribution in a turbulent flow. Izv. Akad. Nauk SSSR Ser. Geogr. Geofiz. 5, 453-466.

OTtino, J. 1989a The kinematics of mixing: stretching, chaos, and transport. Cambridge University Press.

Отtino, J. $1989 b$ The mixing of fluids. Sci. Am. 260, 56-67.

Ouellette, N. T. 2006 Probing the statistical structure of turbulence with measurements of tracer particle tracks. PhD thesis, Cornell University.

Ouellette, N. T., Xu, H. \& Bodenschatz, E. $2006 a$ A quantitative study of threedimensional lagrangian particle tracking algorithms. Exp. Fluids 40, 301-313.

Ouellette, N. T., Xu, H. \& Bodenschatz, E. 2009 Bulk turbulence in dilute polymer solutions. J. Fluid Mech. 629, 375-385.

Ouellette, N. T., Xu, H., Bourgoin, M. \& Bodenschatz, E. $2006 b$ An experimental study of turbulent relative dispersion models. New J. Phys. 8, 109.

Pope, S. B. 2000 Turbulent Flows. Cambridge University Press.

Procaccia, I., L'Vov, V. S. \& BenZI, R. 2008 Theory of drag reduction by polymer in wall-bounded turbulence. Rev. Mod. Phys. 80, 225-247. 
Pumir, A., Bodenschatz, E. \& Xu, H. 2013 Tetrahedron deformation and alignment of perceived vorticity and strain in a turbulent flow. Phys. Fluids 25, 035101.

Pumir, A., Shraiman, B. \& Chertkov, M. 2000 Geometry of lagrangian dispersion in turbulence. Phys. Rev. Lett. 85, 5324-5327.

Pumir, A., Shraiman, B. \& Chertkov, M. 2001 The lagrangian view of energy transfer in turbulent flow. Europhys. Lett. 56, 379-385.

REYNOLDS, O. 1883 An experimental investigation of the circumstances which determine whether the motion of water shall be direct or sinuous, and of the law of resistance in parallel channels. Phil. Trans. Roy. Soc. Lond. A 174, 935-982.

REYNOLDS, O. 1895 On the dynamical theory of incompressible viscous fluids and the determination of the criterion. Phil. Trans. Roy. Soc. Lond. A 186, 123-164.

RICHARDSON, L. F. 1922 Weather prediction by numerical process. Cambridge University Press.

RiCHARDSON, L. F. 1926 Atmospheric diffusion shown on a distance-neighbour graph. Proc. Roy. Soc. Lond. A 110, 709-737.

SAlazAR, J. P. \& Collins, L. R. 2009 Two-particle dispersion in isotropic turbulent flows. Annu. Rev. Fluid Mech. 41, 405-432.

SAWford, B. L. 2001 Turbulent relative dispersion. Annu. Rev. Fluid Mech. 33, 289-317.

SAwford, B. L., Yeung, P. K. \& Borgas, M. S. 2005 Comparison of backwards and forwards relative dispersion in turbulence. Phys. Fluids 17, 095109.

SHAw, R. A. 2003 Particle-turbulence interactions in atmospheric clouds. Annu. Rev. Fluid Mech. 35, 183-227.

Shraiman, B. \& Siggia, E. 2000 Scalar turbulence. Nature 405, 639-646.

Siebert, H., Franke, H., Lehmann, K., Maser, R., Saw, E. W., Schell, D., Shaw, R. A. \& WendisCh, M. 2006 Probing finescale dynamics and microphysics of clouds with helicopter-borne measurements. Bull. Amer. Meteor. Soc. 87, 1727-1738.

Sмith, D. E., BАвсоск, H. P. \& ChU, S. 1999 Single-polymer dynamics in steady shear flow. Science 283, 1724-1727.

SREenivasan, K. R. 1995 On the universality of the kolmogorov constant. Phys. Fluids 7, $2778-2784$.

Stokes, G. G. 1845 On the theories of the internal friction of fluids in motion, and of the equilibrium and motion of elastic solids. Tran. Camb. Phil. Soc. 8, 287-305.

Tabeling, P. 2002 Two-dimensional turbulence: a physicist approach. Phys. Rep. 362, $1-62$. 
TABOR, M. \& De Gennes, P. G. 1986 A cascade theory of drag reduction. Europhys. Lett. 2, 519-522.

TAYLOR, G. I. 1922 Diffusion by continuous movements. Proc. Lond. Math. Soc. 2, 196212.

TAYLOR, G. I. 1935 Statistical theory of turbulence. Proc. Roy. Soc. Lond. A 151, 421-444.

Thomson, D. J. 2003 Dispersion of particle pairs and decay of scalar fields in isotropic turbulence. Phys. Fluids 15, 801-813.

Toms, B. 1948 Some observations on the flow of linear polymer solutions through straight tubes at large reynolds numbers. In Proceedings of the 1st International Congress on Rheology, , vol. 2, pp. 135-141.

Tong, P., GoldBurg, W. I. \& HuAnG, J. S. 1992 Measured effects of polymer additives on turbulent-velocity flutuations at various length scales. Phys. Rev. A 45, 7231-7241.

TOSCHI, F. \& BODENSCHATZ, E. 2009 Lagrangian properties of particles in turbulence. Annu. Rev. Fluid Mech. 41, 375-404.

TSAI, R. Y. 1987 A versatile camera calibration technique for high-accuracy 3D machine vision metrology using off-the-shelf TV cameras and lenses. IEEE J. Robotic. Atom. 3, $323-344$.

VIRK, P. S. 1975 Drag rduction fundamentals. AIChE J. 21, 625-656.

Von KÁRmÁn, T. \& HowARTH, L. 1938 On the statistical theory of isotropic turbulence. Proc. Roy. Soc. Lond. A 164, 192-215.

Vosskuhle, M., LÉvèque, E., Wilkinson, M. \& Pumir, A. 2013 Multiple collisions in turbulent flows. Phys. Rev. E 88, 063008.

Voth, G., la Porta, A., Crawford, A., Alexander, J. \& Bodenschatz, E. 2002 Measurement of particle accelerations in fully developed turbulence. J. Fluid Mech. 469, $121-160$.

WesterweEl, J. 1993 Digital particle image velocimetry: Theory and application. PhD thesis, Technische Universiteit Delft.

White, C. M. \& Mungal, M. G. 2008 Mechanics and predictions of turbulent drag reduction with polymer additives. Annu. Rev. Fluid Mech. 40, 235-256.

Xi, H.-D., Bodenschatz, E. \& XU, H. 2013 Elastic energy flux by flexible polymers in fluid turbulence. Phys. Rev. Lett. 111, 024501.

XU, H. 2008 Tracking lagrangian trajectories in position-velocity space. Meas. Sci. Technol. 19, 075105. 
Xu, H., Ouellette, N. T. \& Bodenschatz, E. 2008 Evolution of geometric structures in intense turbulence. New J. Phys. 10, 013012.

Xu, H., Pumir, A. \& Bodenschatz, E. 2011 The pirouette effect in turbulent flows. Nature Phys. 7, 709-712.

Xu, H., Pumir, A., Falkovich, G., Bodenschatz, E., Shats, M., Xia, H., FranCois, N. \& Boffetta, G. 2014 Flight-crash events in turbulence. Proc. Natl. Acad. Sci. USA 111, 7558-7563.

YEUnG, P. K. 2002 Lagrangian investigation of turbulence. Annu. Rev. Fluid Mech. 34, $115-142$.

Yu, H., Kanov, K., Perelman, E., Graham, J., Frederix, E., Burns, R., Szalay, A., Eyink, G. \& Meneveau, C. 2012 Studying Lagrangian dynamics of turbulence using on-demand fluid particle tracking in a public turbulence database. J. Turbul. 13, 12.

ZIMm, B. H. 1956 Dynamics of polymer molecules in dilute solutions: Viscoelasticity, flow birefringence and dielectric loss. J. Chem. Phys. 24, 269-278. 



\section{Curriculum Vitae}

\section{Personal Information}

Name

Jennifer Jucha, née Mutschall

Date of birth

June 24, 1987

Nationality

German

\section{Academic Background}

04/2011-dato Doctoral studies in Physics

Max Planck Institute for Dynamics and Self-Organization, Göttingen

Georg-August-Universität, Göttingen

International Max Planck Research School for Physics of Biological and Complex Systems (IMPRS PBC)

08/2008-10/2008 Research Internship

Institute Laue-Langevin, Grenoble, France

04/2006-04/2011 Diploma studies in Physics

Johannes Gutenberg-Universität, Mainz

03/2006 Abitur

Hilda-Gymnasium, Koblenz

\section{Publications}

J. Jucha, H. Xu, A. Pumir, E. Bodenschatz. 2014 Time-reversal-symmetry breaking in turbulence. Phys. Rev. Lett. 113, 054501.

J.Jucha, H.Xu, A. Pumir, E. Bodenschatz. The Effect of Polymers on the time asymmetry in pair dispersion. (in preparation) 


\section{Awards and Grants}

$11 / 2012$

APS travel grant for the APS annual meeting

$03 / 2012$

Travel grant from the IMPRS PBC for the program New Directions in Turbulence at the Kavli Institute for Theoretical Physics China

05/2012-04/2014 Fellowship from the IMPRS PBC

$03 / 2006$

School award of the German Physics Society for excellent accomplishments in physics

\section{Teaching Certificate}

10/2013-dato Göttinger Zertifikatsprogramm (exp. completion 10/2014)

Division of Teaching and Learning in Higher Education

Georg-August-Universität, Göttingen

The certificate program consists of several action-oriented modules aimed at advancing the participant's teaching skills. It is concluded by the preparation of a personal teaching portfolio.

\section{Teaching Experience}

10/2012-dato Lecturer, Georg-August-Universität, Göttingen

$\diamond$ Seminar "Current questions in turbulence research"

08/2011-10/2012 Teaching assistant, Georg-August-Universität, Göttingen

$\diamond$ Thermodynamics and statistical mechanics

$\diamond$ Quantum mechanics

10/2008-07/2010 Teaching assistant, Johannes Gutenberg-Universität, Mainz

$\diamond$ Classical mechanics

$\diamond$ Electrodynamics and classical field theory

$\diamond$ Computational physics (several iterations)

$\diamond$ Advanced undergraduate lab course (several iterations)

$\diamond$ Graduate lab course 


\section{Talks and Conferences}

$02 / 2014$

$11 / 2013$

$09 / 2013$

$07 / 2013$

03/2012-04/2012 Program New Directions in Turbulence

Talk at the Scientific Advisory Board Meeting

MPI for Dynamics and Self-Organization, Göttingen

"Time-Irreversibility of Lagrangian Statistics in a Turbulent Flow"

International Workshop on Turbulence and Amorphous Materials

Eilat, Israel

"Time Asymmetry in Relative Pair Dispersion"

European Turbulence Conference (ETC14)

École Normale Supérieur Lyon, Lyon, France

"Deformation of Tetrahedra in Turbulence"

"The Effect of Polymers on the Time Asymmetry in Pair Dispersion"

Annual Meeting of the APS Fluid Dynamics Section

San Diego, United States of America

"Shape Deformation of Tetrahedra in Fully Developed Turbulence"

\author{
Kavli Institute for Theoretical Physics China, Beijing, China \\ "Shape Deformation of Tetrahedra in Fully Developed Turbulence" \\ 04/2011-06/2011 Program The Nature of Turbulence \\ Kavli Institute for Theoretical Physics, Santa Barbara, USA
}

Göttingen, September 3, 2014 



\section{Acknowledgments}

First of all, I would like to thank my supervisor Eberhard Bodenschatz for offering me the possibility to conduct my doctoral research in such a stimulating and supporting environment. With his enthusiasm for new ideas and projects he greatly motivated me to think outside the box and dare to try new things. What I appreciate most is the guidance he provided for my personal and professional development. He helped me to find out which career I want to pursue and enabled me to work towards my goals.

I also thank Annette Zippelius and Jens Niemeyer for their helpful ideas and recommendations during my thesis committee meetings. Being from different fields of research, they contributed the needed outside view on my project and helped me to prevent blind spots.

Another person contributing a lot to the success of this thesis is Haitao Xu. With his seemingly endless knowledge on experimental techniques and his great patience in teaching them he was the one to support me in my challenging transition from a theoretical to an experimental physicist. His great experience on turbulent flows and our many intense discussions on experimental findings were an enormous help in interpreting my data. His door was always open for me and I am deeply grateful for this.

I also want to thank my collaborator Alain Pumir from École Normale Supérieure, Lyon. Thanks to him I was able to compare my experimental findings with numerical results, and even tackle some questions which were not accessible to my data. Beside this, he was always willing to double-check and improve my calculations, and was never tired of explaining some of the more complex theoretical ideas to me.

Further theoretical support was provided by Anna Frishmann from the Weizmann Institute of Science in Rehovot. After I discussed with her my puzzling finding that the odd and even terms of my short-time expansion seem to have a different intrinsic timespan, she quickly developed the brilliant proof on why this must be the case. I also greatly appreciate our discussions on science and culture which made many a conference more enjoyable.

Of course, I also want to thank all the lovely people at the institute who made my stay here so great. I very much enjoyed the friendly atmosphere and the readiness to help each other. I would like to especially thank Garrett Good for proofreading every line of this thesis at least once and helping me to improve my English. I'm also very grateful towards all the excellent 
technicians working in the mechanics and electronics workshops and the experimental hall. Their help in all my small and large disasters in the lab is invaluable.

Great thanks are also due for the whole GGNB team. It is due to their commitment that the graduate school is as unbureaucratic and supporting as we know it.

Last but not least I would like to thank my family for their love and support in all these years. I am very happy to have such loving parents who always encouraged me to do what I enjoy and never failed to offer help or advice. I also thank my sister for always listening to my problems and putting them into the right perspective. Finally, I would like to thank my husband Patrick. His deep trust in me and his ability to always make me laugh even made the most difficult times enjoyable. 